\title{
Programs to Estimate UV Dosage and Damage
}

Brian Dickens

Eric Byrd

This publication is available free of charge from:

https://doi.org/10.6028/NIST.IR.7500

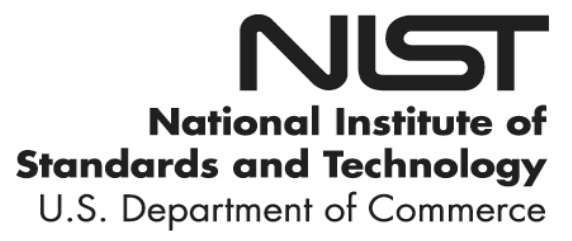




\section{Programs to Estimate UV Dosage and Damage}

Brian Dickens

Eric Byrd

Prepared for Jonathan Martin and Tinh Nguyen

Building and Fire Research Laboratory

National Institute of Standards and Technology

Gaithersburg MD 20899

September, 1999

This publication is available free of charge from:

https://doi.org/10.6028/NIST.IR.7500

June 2008

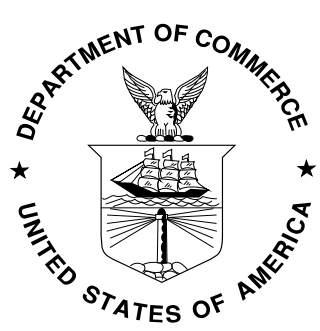

U.S. Department of Commerce Carlos M. Gutierrez, Secretary

National Institute of Standards and Technology James M. Turner, Deputy Director 


\section{Guide to using \\ Programs to Estimate UV Dosage and Damage}

Brian Dickens and Eric Byrd

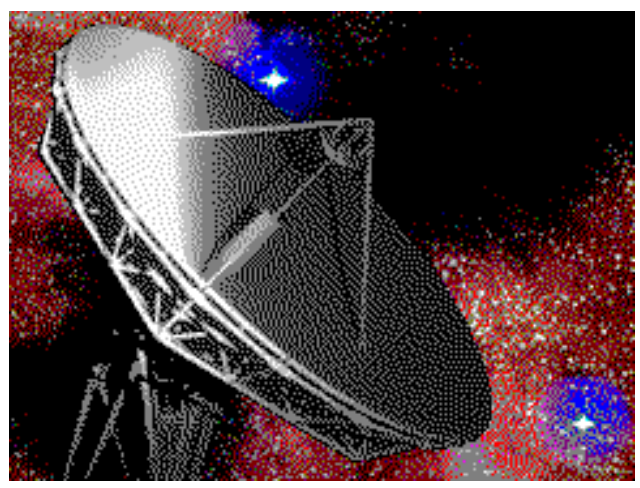

Prepared for Jonathan Martin and Tinh Nguyen

Building and Fire Research Laboratory National Institute of Standards and Technology Gaithersburg MD 20899 September, 1999 


\section{Introduction}

The system of programs described in this guide is concerned with estimating the damage ensuing from exposure of specimens in dry and humid atmospheres to UV and visible radiation covering the solar range. Because damage occurring during such exposures can arise from both photolytic degradation and hydrolysis (and perhaps other "dark" reactions), provision has been made to separate these effects.

Photolytic damage is caused by energy absorbed by the specimen from sunlight or similar radiation incident on the specimen. Photolytic damage is expected to be wavelength specific, partly because different wavelengths contain different amounts of energy per photon and partly because which chemical group acts as the chromophore depends on the wavelength of the incident radiation. Photolytic damage is instantaneous following the absorption of radiation.

Hydrolysis is attainment of a temperature-dependent equilibrium specified by the concentrations of hydrolysable groups and water in the material (typically expressed in moles/liter). Because hydrolysis is a relatively slow process, it can take days or weeks for equilibrium to be reached.

In the experiments carried out to date, damage has been monitored quantitatively by changes in IR spectra. This has the advantage of providing information on the chemical processes involved. Other quantitative evidence of damage will be used in future investigations as the need arises but the current emphasis in this system of programs is on correlation of photodegradation and absorbed radiation.

\section{Dose may be defined as the total amount of radiation incident on a} specimen.

The (absorbed?) dosage may be defined as that part of the dose which is absorbed by the specimen.

The effective dosage is then that part of the dosage which causes the damage under consideration.

As defined, the effective dosage is the integrated product of the dosage and the quantum yields, the fraction of events which lead to damage.

The dose is estimated from UV spectra of the lamps and the interference filters (used to isolate a particular wavelength range). The dosage is estimated from the dose and the UV absorption of the specimens themselves. Some of these quantities changed with time. For example, the lamps aged and the specimens degraded. The experimental procedure was therefore to use the appropriate UV-visible spectra to monitor changes over short ranges of time over which any change is presumed to be linear. Once the output of the lamp and the 
transmittance of the filter are known, the amount of radiation incident on a specimen, the dose, can be estimated.

From the transmittance of the specimen, the specimen absorbance can be calculated. Radiation incident on the specimen but which does not emerge at the other side of the specimen is presumed to have been absorbed by the specimen and is taken as being the apparent dosage. Whether to consider that all the absorbed radiation leads to degradation or whether some radiation is considered to be "harmlessly" absorbed by degradation products rather than by the original coating material is a choice offered by the programs.

The current procedure was developed for transparent specimens. For opaque specimens, absorptance will be estimated from a combination of reflectance and transmittance measurements

These programs allow rapid estimation of dosage and damage from legions of data. In fact, one could say that the programs make possible the processing of so much data. This in turn makes feasible a systematic study of the effects of the "stress" factors over wide ranges of stress, a desirable and probably necessary condition where correlated processes are to be resolved. The programs are also research tools that organize the data so that aspects of the experiments can easily be recalled and examined. Archiving and copying the data merely require copying a few database tables. A more complete description is given in the Overview.

The programs have been designed to be complete yet relatively easy to use. They run under 32-bit Windows, i.e., Windows 95, Windows 98 and Windows NT. The file structure of the database tables is MS FoxPro. This structure was chosen because it is widespread, has each table in a physically different file and is therefore quite flexible, and allows the easy manipulation of so-called memos or "blobs" - collections of binary data which can be stored compactly on the computer disk yet can be read into computer memory extremely quickly.

When first approaching the programs, one should bear in mind that, although the desire throughout industry is for quick, cheap, simple and dirty yet completely relevant tests, such tests must concentrate on the most important physical and chemical parameters. That can only be done in a confident fashion when the response of a material to a wide range of factors has been established so that there is high probability that the most important factors have been found. Anything less is a simple guess. These programs allow manipulation and processing of the massive amounts of data required to carry out such comprehensive tests. It is a good idea to leave such tasks to the computer.

\section{Overview}

The BFRL photoreactor was carefully designed to allow exposure of specimens to a wide range of factors that are potentially important in degradation of the specimens and to measure the exposure conditions and the ensuing degradation. The photoreactor will be augmented to 
provide greater and greater capability as the range of specimens and conditions diversify.

Together, the photoreactor and the software provide the following capabilities:

- Generate and handle data for a wide range of exposure conditions

- highly automated apparatus

- examine precision of data

- impose discipline on experimental protocols

- automate expertise

- provide checks for gross errors in new data

- allow rapid assessment of when enough data have been collected and the experiments can be terminated

- archival storage of data in readily accessible form

- greater productivity

Enormous capability now exists. Nonetheless, the programs and experimental protocols will undoubtedly evolve as the experiments demand more intricate analysis and greater automation.

It should be kept in mind that:

- $\quad$ service life prediction is often extrapolation of trends

- how far results can be extrapolated depends on constraints in the model and on the quality of the data

- subtraction and multiplication increase the noise in the derived quantities

- concurrent hydrolysis and photolysis require high precision data so that the primary effects AND their interaction(s) can be isolated

- the experimental measurements must be precise over the duration of the experiments, which up to now has been 3-6 months

- the spectral resolution must be sufficient to represent the filter profiles

- degradation products may shield the parent matrix from UV 
- damage may be wavelength specific as far as concerns which chemical reactions ensue and what the quantum yield is for each reaction

- multiwavelength studies may be required if new chromophores are produced during degradation.

The photoreactor has provided good working protocols dealing with

- experimental design

- filter ranges

- length of exposure

- light without heat

- light collimation/delivery to cells

- temperature and humidity ranges (vapor pressure ranges)

- temperature and humidity control

- window materials being transparent and humidityproof

- thickness of specimens

- anticipating and monitoring changes in apparatus

- methods of measuring spectra

The design of the computer programs necessitated the following considerations:

- design of database tables - what information is necessary and where to put it

- data entry programs - how to enter information into the tables

- what to do about missing data

- finding errors in the data

- $\quad$ processing data to get the relevant spectra

- correcting for non-zero baseline, ablation/erosion, and dark reactions

- where to look in damage spectra to assess the damage

- estimating quantum yields when the damage/dosage curves are not linear

- showing the results

- allowing exploration of the experimental evidence in ways needed to unravel the complexities in the relationships between effects 


\section{Estimation of dosage, damage and quantum yield}

The following brief overview is provided so that the workings of the programs will be evident.

\section{Dosage}

Radiation from the lamp is directed to the cell containing the specimen, where it passes through a filter and is then incident on the specimen. The specimen absorption spectrum gives the fraction of the incident radiation that emerges from the other side of the specimen.

Components such as the lamps and specimens change with time. These changes are followed by taking UV-visible spectra and, in the case of the specimens, IR spectra, at reasonable intervals.

The procedure followed to estimate the dosage is:

Lamp spectra: interpolate between spectra adjacent in time to get the output, $\mathrm{I}_{0}$, of the lamp in watts

Filter spectra: interpolate between spectra adjacent in time to get $\%$ transmittance $=\mathrm{f}$.

The radiation transmitted through the filter, $I_{f}$, is given by $I_{f}=\mathrm{fl}_{0} / 100$

Specimen spectra (absorbance): transmitted radiation $I_{t}=I_{f} 10^{-A}$ where $\mathrm{A}=$ absorbance. Interpolate between spectra adjacent in time to get $\mathrm{A}$ at each wavelength for which the spectra were measured.

The radiation in watts absorbed by the specimen then given by $I_{f}-I_{t}=I_{f}\left(1-10^{-A}\right)=f I_{0} / 100\left(1-10^{-A}\right)$. Because $I_{0}$ is in watts, this is the flux the specimen receives (in Joules/s). It is wavelength-dependent. Multiplying the flux by the elapsed time in seconds gives the dosage, i.e., the energy the specimen has absorbed, in Joules.

\section{Propagation of error}

A model must successfully account for effects seen in the experiments before it can be used to generate prophesies. But before a model can be proposed, the effects must be visible, i.e., above the noise. The noise levels in the measurements are very important, first in determining whether or not the effects of the experiments are discernible and second in determining the applicable range (in time or radiation level or hydrolysis level) of any prophesy.

These programs and computational procedures involve multiplying, dividing and subtracting combinations of spectral absorbances. It is 
necessary to minimize the noise produced in the measuring processes in order not to be disappointed in the results.

Given that $z=a+b x+c y$, then the propagation of error formula for subtraction and addition of $x$ and $y$ to yield $z$ is

$\sigma^{2}(z)=b^{2} \sigma^{2}(x)+c^{2} \sigma^{2}(y)$, where $\sigma$ is the standard error in the quantity.

For multiplication/division, $z=x^{a} y^{b}$, the formula is

$\sigma^{2}(z) / z^{2}=a^{2} \sigma^{2}(x) / x^{2}+b^{2} \sigma^{2}(y) / y^{2}$

Clearly, noise is never lost. Subtraction and division are especially troublesome in that the new derived quantity is usually smaller than the original quantities but the noise of the derived quantity is always larger than the noise in either of the original quantities.

\section{Damage}

In the photolytic part of the damage suffered by the specimen, the damage at a particular wavelength of incident radiation is given by

damage $=$ Flux ${ }^{*}$ time ${ }^{*}$ quantum yield (at each wavelength in the spectra)

and the dosage, $D$, is given by $D=$ Flux * time.

Flux and time are quantities from the exposure in the photoreactor. The flux is assumed to be constant over the time under consideration and the time between measurements has to be made sufficiently short (perhaps a week) that the assumption is valid.

If the damage is represented in terms of dosage by a polynomial, then

damage $=a_{0}+a_{1}{ }^{*} D+a_{2}{ }^{*} D^{2}+a_{3}{ }^{*} D^{3}+a_{4}{ }^{*} D^{4}$

where $a_{0}, a_{1}, a_{2}, a_{3}$ and $a_{4}$ are coefficients of the polynomial and $D$ is the dosage.

A polynomial is convenient for interpolation because it allows for any reasonable shape of damage/dosage curve, including those which are non-linear with increasing dosage due to shielding of the matrix by degradation products, using up weak links and/or chromophores, etc. A polynomial should not be used for extrapolation. 


\section{Quantum yield}

Quantum yield $(q)$ is the damage per unit dosage and is wavelengthspecific. As defined, quantum yield is the probability of an absorbed photon causing the damage but putting the damage on an this scale requires molar information which is rarely available for cross-linked solid polymerized specimens. The units of quantum yield used in these programs are damage per Joule of dosage or damage per photon of dosage depending on the options chosen. Damage is the units of the damage spectra.

The quantum yield for a particular wavelength in the incident radiation is given by differentiating equation (1) to give

$d($ damage $) / d D=a_{1}+2^{*} a_{2}{ }^{*} D+3{ }^{*} a_{3}{ }^{*} D^{2}+4^{*} a_{4}{ }^{*} D^{3}$.

The coefficients $a_{1}$ etc., propagate the random errors arising from

1) the experimental data and

2) the assumption that the polynomial describes the damage/dosage relationship.

\section{Systematic errors are not discerned in this treatment.}

For the simple case where damage is linear with time,

Damage $=$ Flux ${ }^{*}$ Time ${ }^{*} \mathrm{q}$.

Flux and Time are quantities characterizing the expected exposure, $q$ is the quantum yield and Damage is the expected damage. From the propagation of errors for multiplication,

$\sigma^{2}$ (Damage $) /$ Damage $^{2}=\sigma^{2}($ Flux $) /$ Flux $^{2}+\sigma^{2}($ Time $) /$ Time $^{2}+\sigma^{2}(\mathrm{q}) / \mathrm{q}^{2}$

Note that the uncertainties in the conditions which characterize the expected service environment play just as important a part in the estimation of the expected damage uncertainty of the result as do the uncertainties in the quantum yields.

\section{Data Structures used in the Programs}

\section{Identification details and experimental data}

The database tables devised for this work represent a compromise between

1) the paperless office and the subsequent freedom from looking for forgotten notebooks and 
2) rigid requirements that even information not particularly germane to the procedure must be entered into the computer.

The general idea is that information shall only be entered in one place. For example, if the center wavelength of the window of transmittance of a filter is found to be different from what was entered in the tables, only the entry for that filter in the filter table has to be changed. All procedures requiring the filter maximum will read the filter table to get this information and will not have to be updated.

The damage table is an exception to this convention. First, it was deemed too time-intensive and too demanding of computer memory to read all the relevant information from the appropriate tables as needed. Second, the copied values are the values the tables contained when the derived quantities were being calculated. If the primary data are changed (most probably in removing some error in the table), the damage tables should be regenerated. Fortunately, the program handles all writing to the damage tables and regenerating them is an easy matter.

For every database table there should be a file with extension dbf. After the file has been indexed (for example, the data processing program has been run), there may be an index file, with extension cdx. These index files are typically regenerated when the program which uses them is rerun (except of course if the files are on a CD-ROM). If the database table contains memos/BLOBS (see Appendix 1), there will be a file with extension fpt. The dbf file has a short entry which points to the right place in the fpt file. If you copy database files, do not forget to copy the fpt file (if there is a fpt file) along with the dbf file. If you are copying to CD_ROM, you will have to copy the cdx files also.

All dates and times are represented as floating point numbers with 4 digits after the decimal point, i.e., 35500.5000 , which is the number of days and fractional days since the beginning of January $1,1900$.

The detailed structure of the database tables is given in Appendix 1.

\section{Programs}

\section{Requirements}

The requirements of the computer programs are to:

- allow the user to put information into the database tables

- handle missing data

- find gross errors in the data

- $\quad$ automate finding spectra in databases

- automate corrections of spectra for non-zero baseline 
- automate corrections for erosion/ablation/specimen thickness

- correct for hydrolysis and other dark reactions

- allow the user to select anywhere in the damage spectra as a site to monitor

- examine correlations between sites in the damage spectra easily

- generate damage/dosage curves

- estimate quantum yields whether the damage/dosage curves are linear or not

- fit a polynomial to the damage/dosage curve

- calculate the derivative of the polynomial to give quantum yield as a function of damage

- show the results

\section{Classes of programs}

The description of the programs has been divided into data entry, processing the data to provide the damage/dosage relationships, and examining the derived quantities in various ways.

\section{Data entry program ShowDBEditors}

The data describing the experiment and the samples are kept in database tables. One of the rules about databases is that information should not be put into a database in more places than is really necessary. This means that a database system consists of a set of tables which refer to each other. For example, most purchased items have a supplier and if two items are purchased from the same supplier, the supplier's address should not be entered into the database twice. Instead, a table of suppliers is used to provide a supplier number keyed to the records in the supplier table. A table is therefore not THE database but is merely part of it. The convention when speaking of databases and tables is that the database is a collection of tables.

\section{Creating database tables}

$$
\begin{gathered}
\text { Shortcut to } \\
\text { ShowDBEdit... }
\end{gathered}
$$

When beginning a new series of exposures, it is necessary to create several new database tables. Other tables, for example the suppliers table and perhaps the lamp power table, can be re-used. Tables are created using the ShowDBEditors program, as shown below. 
을 BFRL Create and Edit Specimen Exposure Database Tables

Edit/Create Database Editors

Edit Suppliers DB Table

Edit Lamp IDs DB Table

$\Gamma$ Edit Filter IDs DE Table

Г Edit Cell IDs DB Table

Г Edit Specimen IDs DE Table

Г Edit Specimens in Cells DB Table

ए Edit Cells In Positions DE Table

$\Gamma$ Edit Lamps On Off DB Table

Г Edit Position Lamp DE Table

Edit Lamp Power DB Table

\begin{tabular}{|c|}
\hline Create New Suppliers DB \\
\hline Create New Lamp IDs DB \\
\hline Create New Filter IDs DE \\
\hline Create New Cell IDs DB \\
\hline Create New Specimen IDs DE \\
\hline Create New Specimen in Cells DE \\
\hline Create New Cells in Positions DE \\
\hline Create New Lamps OniOff DE \\
\hline Create New Position To Lamp DE \\
\hline Create New Lamp Power DB \\
\hline
\end{tabular}

\begin{tabular}{|c|}
\hline Assign/Change Suppliers DB \\
\hline Assign/Change Lamp IDs DB \\
\hline Assign/Change Filter IDs DE \\
\hline Assign/Change Cell IDs DB \\
\hline Assign/Change Specimen IDs DB \\
\hline Assign/Change Specimen in Cells DB \\
\hline Assign/Change Cells in Positions DE \\
\hline Assign/Change Lamps On/Off DB \\
\hline Assign/Change Position To Lamp DB \\
\hline Assign/Change Lamp Power DB \\
\hline
\end{tabular}

Make a new Suppliers Database Table

If we want to create a new suppliers table, we first click on the "Create New Suppliers DB Table".

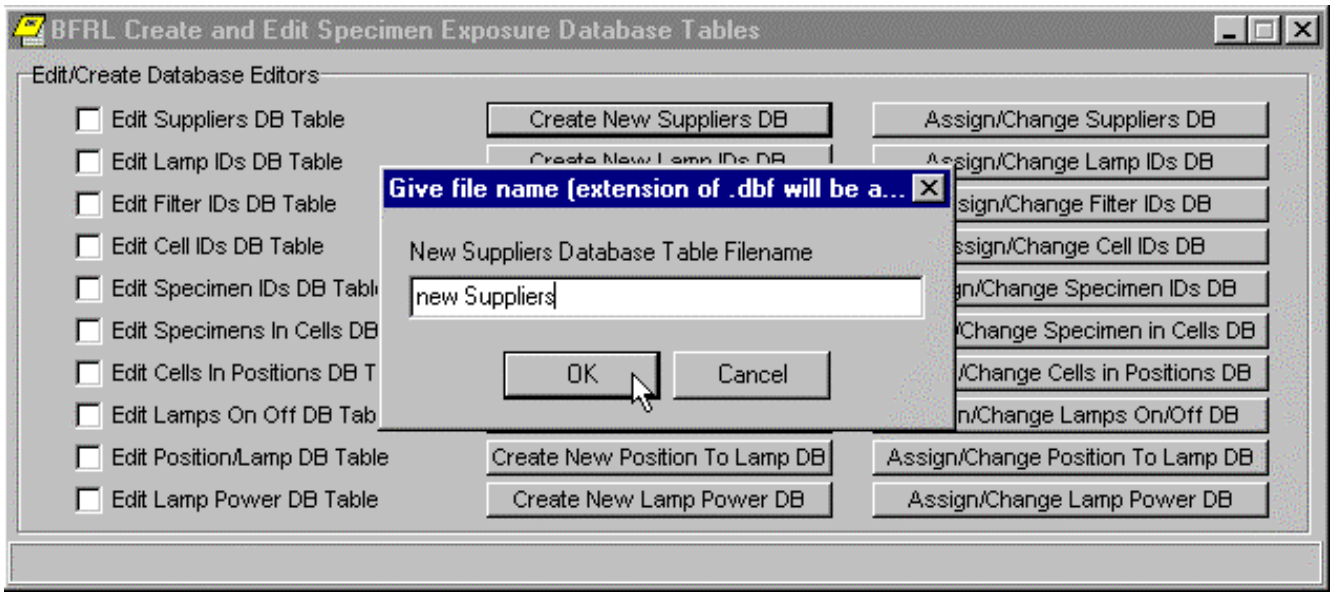

The clicking action brings up a dialog box where the name of the new suppliers database table can be typed. Since long files names are allowed, a recognizable file name can be used. Clicking on OK causes the program to try to create the database table. Clicking on Cancel aborts the procedure.

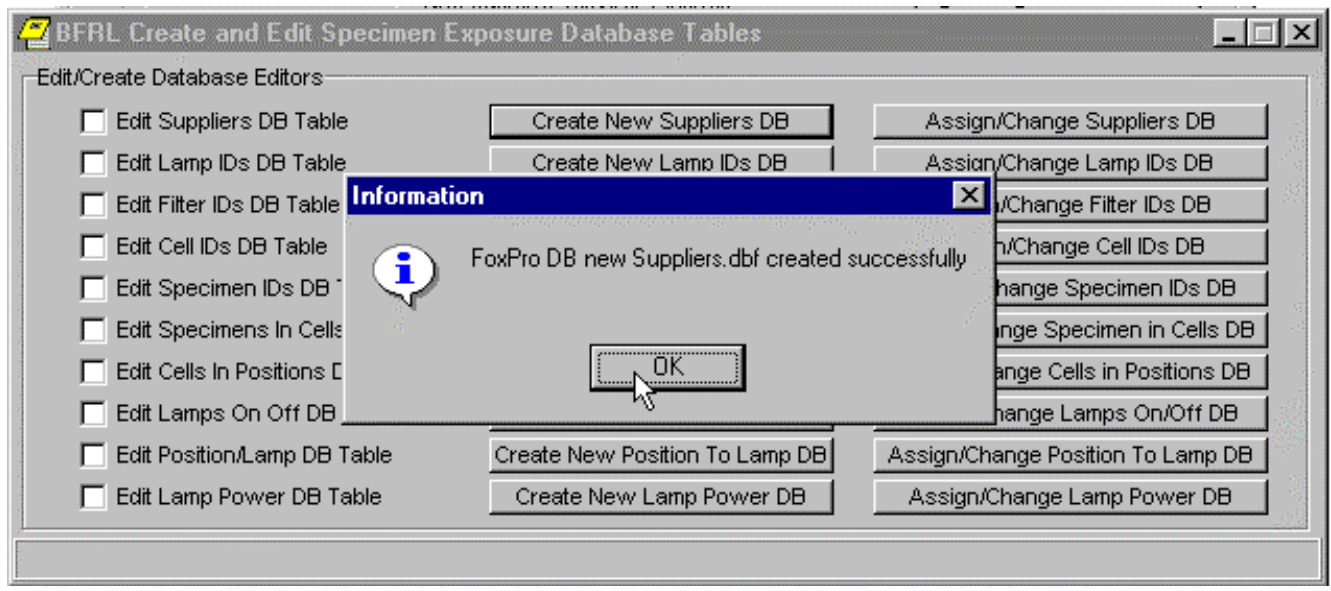

A dialog box informs the user whether or not the table was created. 


\section{Filling the database tables}

The table must then be filled with information. This is begun by checking the "Edit Suppliers DB" check box. If the table already exists but has not been assigned to the program in the current run, the following dialog box will be shown.

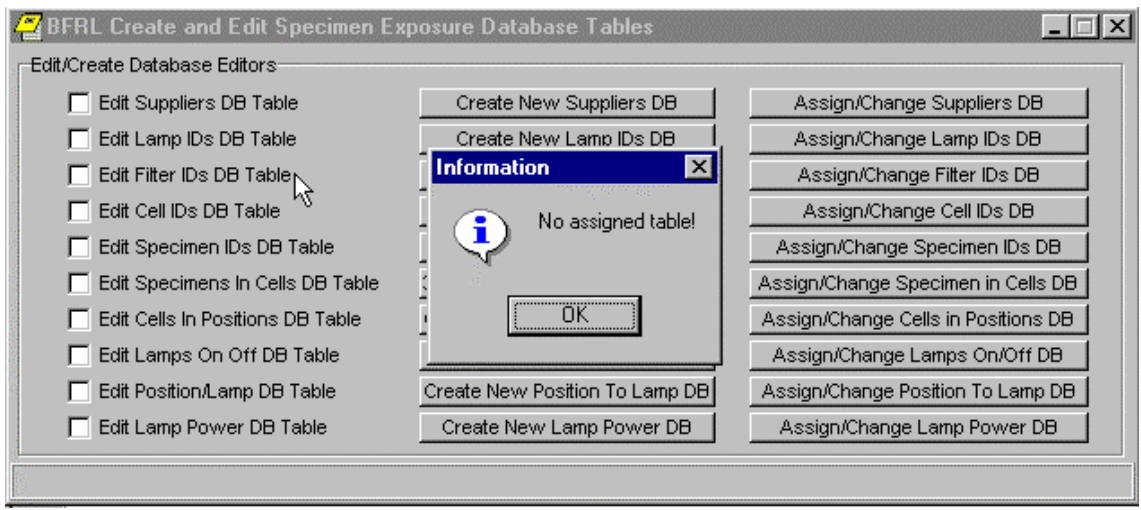

Close the dialog box using the OK button or the $x$ in the upper right hand corner. Then click on the "Assign/Change Suppliers DB" button.

\begin{tabular}{|c|c|c|}
\hline \multicolumn{3}{|c|}{ 을 BFRL Create and Edit Specimen Exposure Database Tables } \\
\hline \multicolumn{3}{|l|}{ Edit/Create Database Editors } \\
\hline Г Edit Suppliers DB Table & CreateNew Sundiersug - & AssigniChange Suppliers DB \\
\hline Г Edit Lamp IDs DE Table & Create New Lamp IDS DB & Assign/Change Lamp IDs DB \\
\hline Г Edit Filter IDs DB Table & Create New Filter IDs DE & Assign/Change Filter IDs DB \\
\hline Г Edit Cell IDs DB Table & Create New Cell IDS DB & AssigniChange Cell IDs DE \\
\hline Г Edit Specimen IDs DB Table & Create New Specimen IDs DB & Assign/Change Specimen IDs DB \\
\hline Г Edit Specimens In Cells DB Table & Create New Specimen in Cells DB & Assign/Change Specimen in Cells DB \\
\hline$\Gamma$ Edit Cells In Positions DB Table & Create New Cells in Positions DE & Assign/Change Cells in Positions DB \\
\hline Г Edit Lamps On Off DB Table & Create New Lamps OniOff DB & Assign/Change Lamps On/Off DB \\
\hline Г Edit Position/amp DE Table & Create New Position To Lamp DE & AssigniChange Position To Lamp DE \\
\hline Г Edit Lamp Power DB Table & Create New Lamp Power DB & AssigniChange Lamp Power DB \\
\hline
\end{tabular}

To change any currently active database table or to assign a database table, as in when newly running the program, click on the appropriate "Assign/Change" button. This brings up a dialog box to allow the table to be read into the program. 


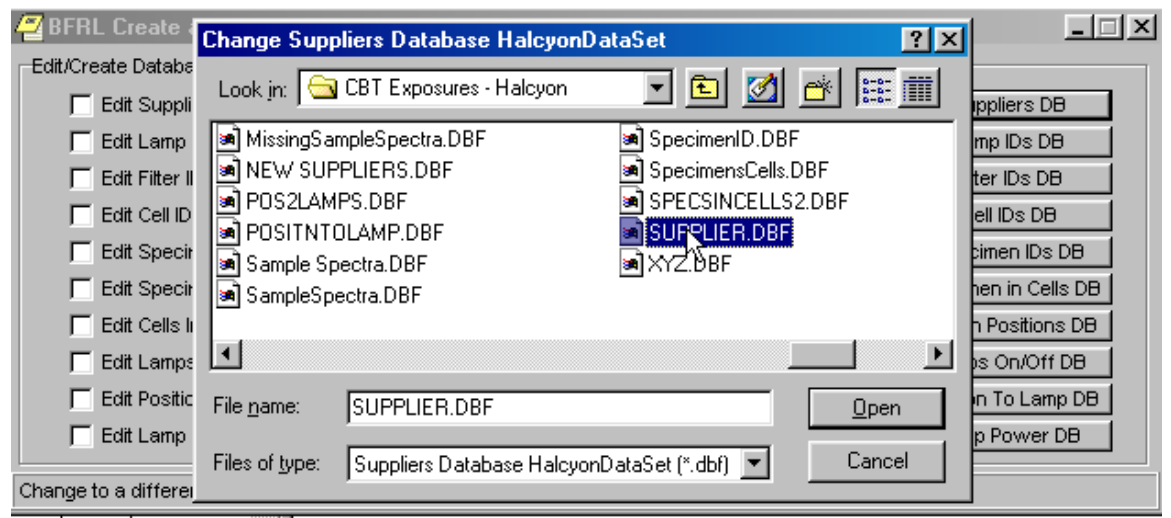

When the table has been assigned to the program, a data entry dialog box below appears. If the table is empty, there will of course be no information in the dialog box.

For example, when the "Edit Suppliers DB Table" check box is checked and the dialog box which allows editing of the Suppliers table appears, the information in the first record in the suppliers database table appears in the Edit Suppliers Database Table window. The information can be edited or augmented. Moving from record to record and creating new records is accomplished using the navigator bar at the top of the window.

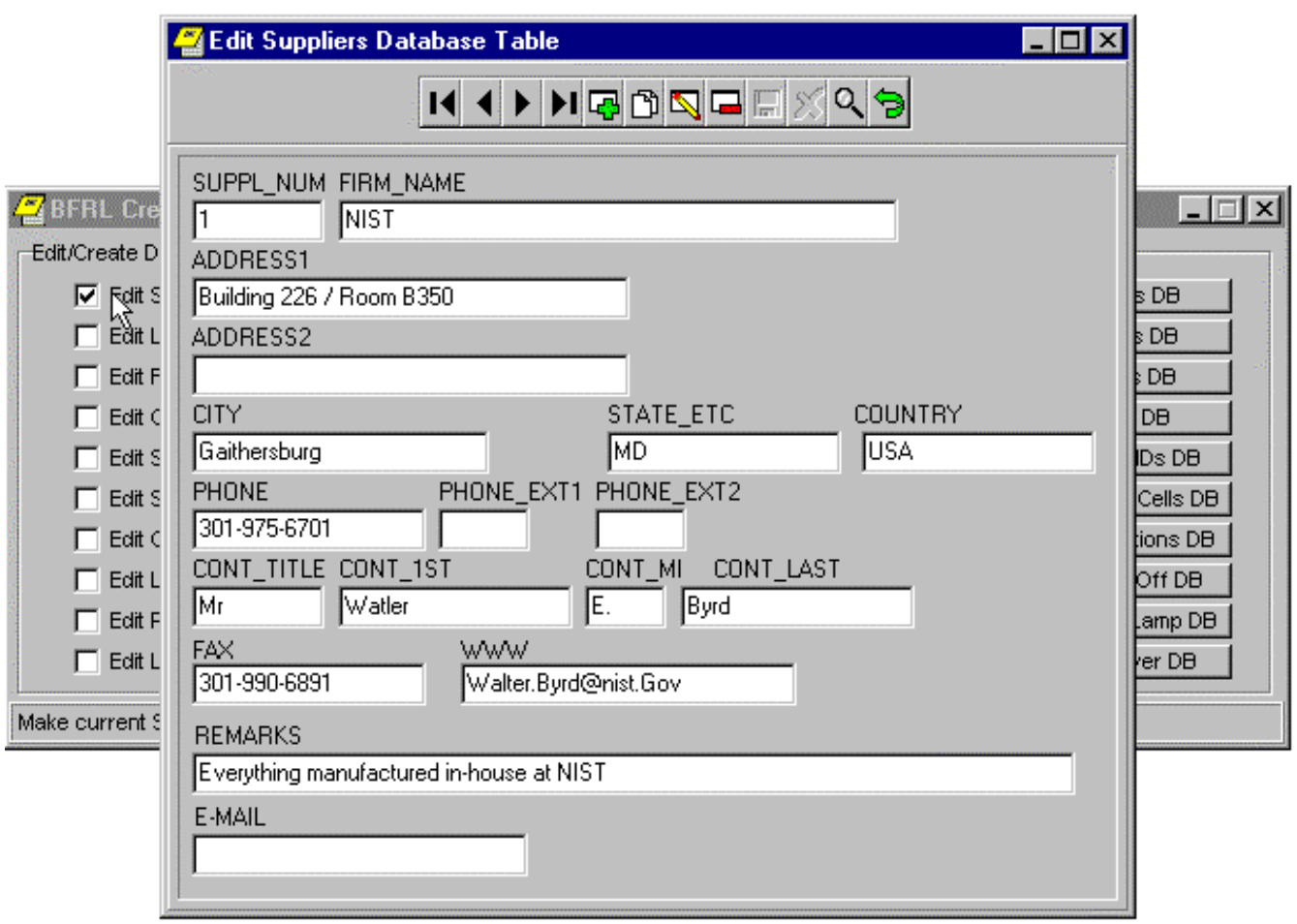

A help hint appears at the bottom of the main window when the mouse cursor is paused over the navigator bar buttons. 


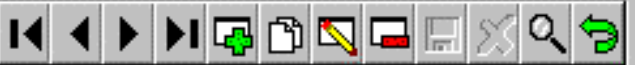

From left to right, these buttons are:

I

go to the beginning of the file (table)

1 go one record towards the beginning

1 go one record towards the end

DI go to the end of the file

可 append a record (at the end of the file)

[9] copy the current record

D. edit the current record

delete the current record

save the current record (after editing) - disabled here because no editing has been done

cancel any changes made during editing - disabled here because no changes have been made.

The other database tables in the system are shown below. The type of table is given in the blue header bar at the top of each window.

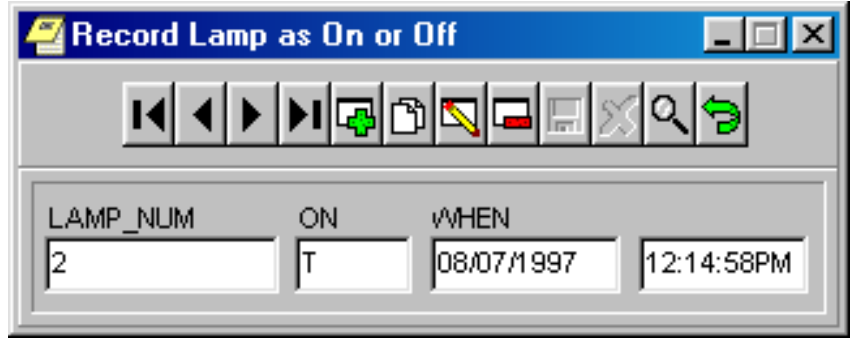


The $T$ means that it was true that the lamp was ON. Setting the lamp to OFF would require that the table states that is was false that the lamp was on. This seems to be a complicated way of designating something which is inherently simple but is a consequence of the restriction that one field has to be used to describe the event.

Dates/times of day are stored in the tables as days and fractions of days, but they are shown to the user as normal dates and times.

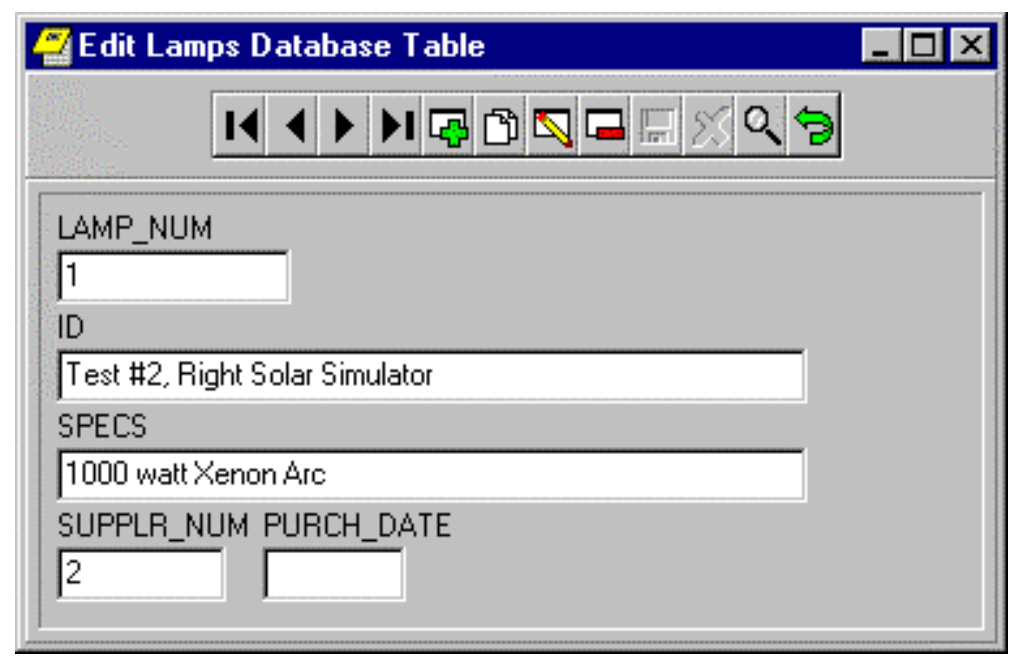

Each lamp must be given a unique number and must also be identified as in the above dialog box. The supplier number can be dragged from the appropriate record in the Suppliers table into the SUPPLR_NUM box. Not all fields must be filled out, as shown by the empty PURCH_DATE box. The field descriptions such as LAMP_NUM and SUPPLR_NUM are those used in the tables themselves and will appear in this way in programs which show the contents of the database tables.

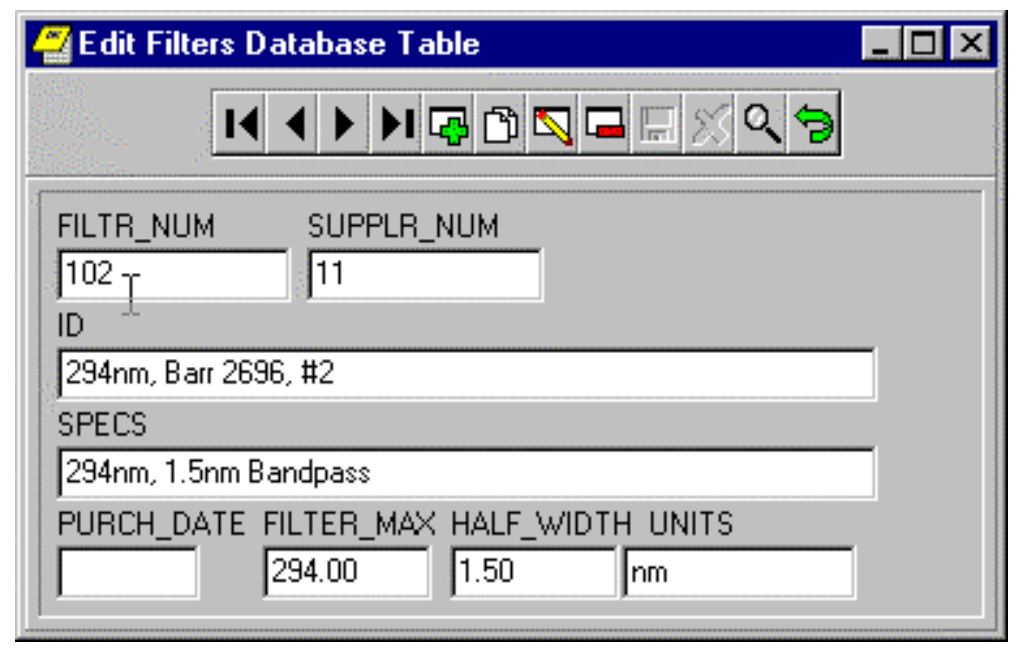

It is important that the FILTER_MAX and HALF_WIDTH fields in the Filter IDs table be filled out because they are used in the data processing program to estimate the range over which to integrate the dosage and to provide a wavelength range over which to verify that the specimen UV-visible absorbance was satisfactorily measured. In the 
visible part of the spectrum, the absorbance of a clear specimen is essentially zero and is poorly known because it is roughly the same level as the noise, but the lamp intensity is very high and the estimated dosage, although considerable, can easily be considerably in error. See later for a description of how either a scaled-down spectrum of a thick specimen or a requirement that the actual absorbance exceed a minimum value can be used to overcome this problem .

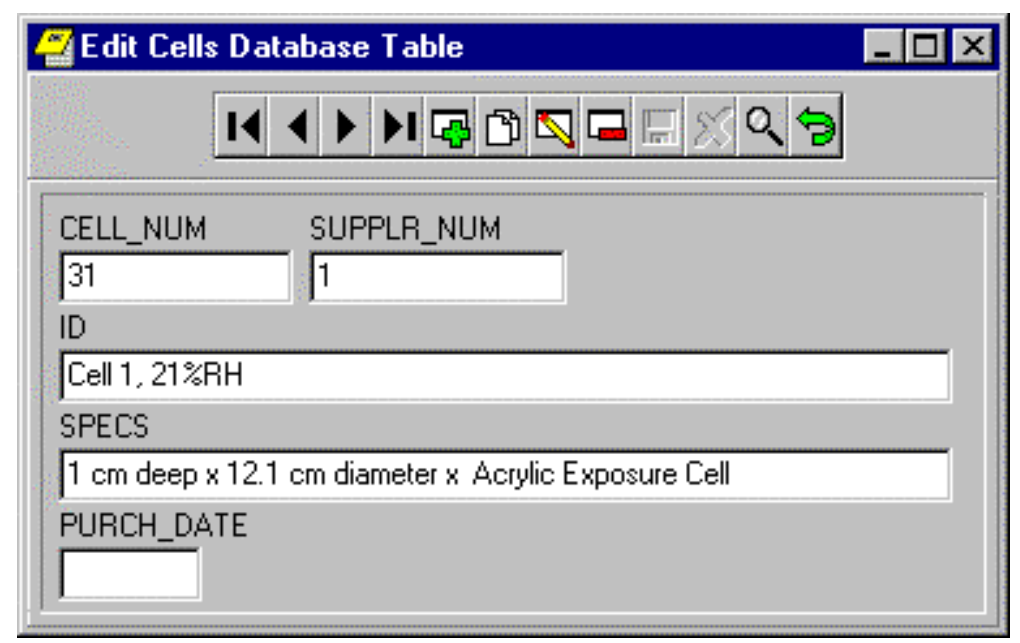

The Cell IDs table is little used but is provided to document from where and when the cells were obtained.

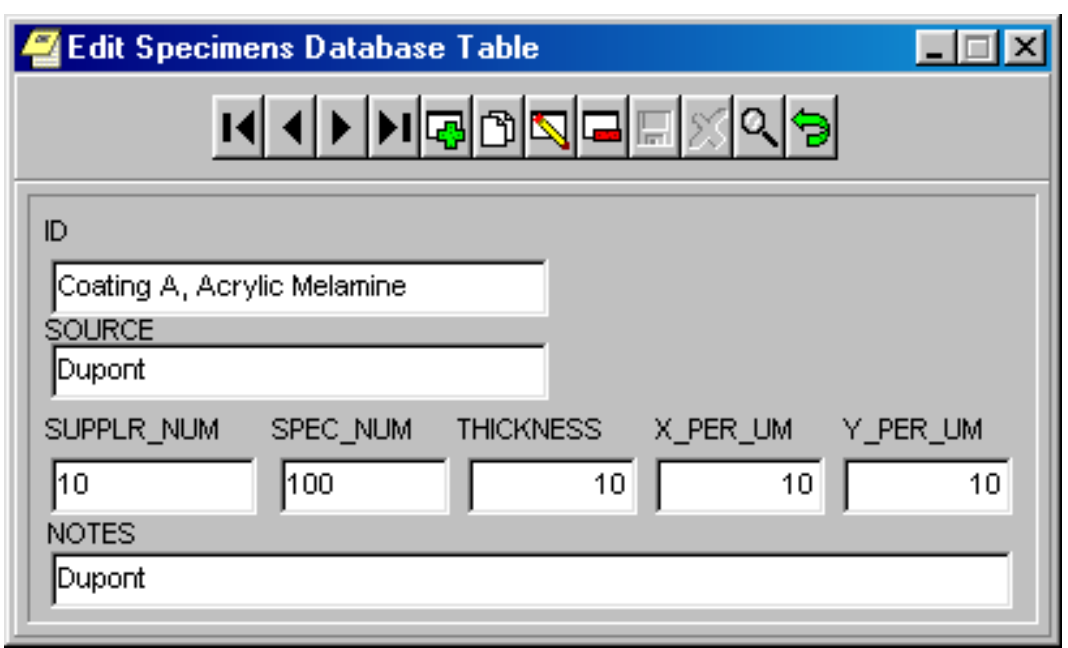

The Specimen IDs table provides the all-important specimen number and specimen thickness. 


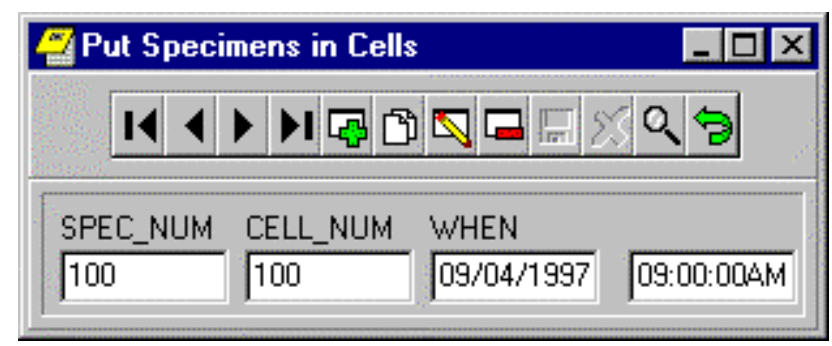

The Specimens in Cells database table ties the specimen to a filter, temperature and humidity through the CELL_NUM connection to the cell position table. The cell position field in the cell position table the ties the cell, and the specimen it contains, to a position in the illumination field. That position is in turn related to a lamp number and to the spectra of the radiation from that lamp which reached the cell position.

The Cell Position table contains the experimental conditions of the

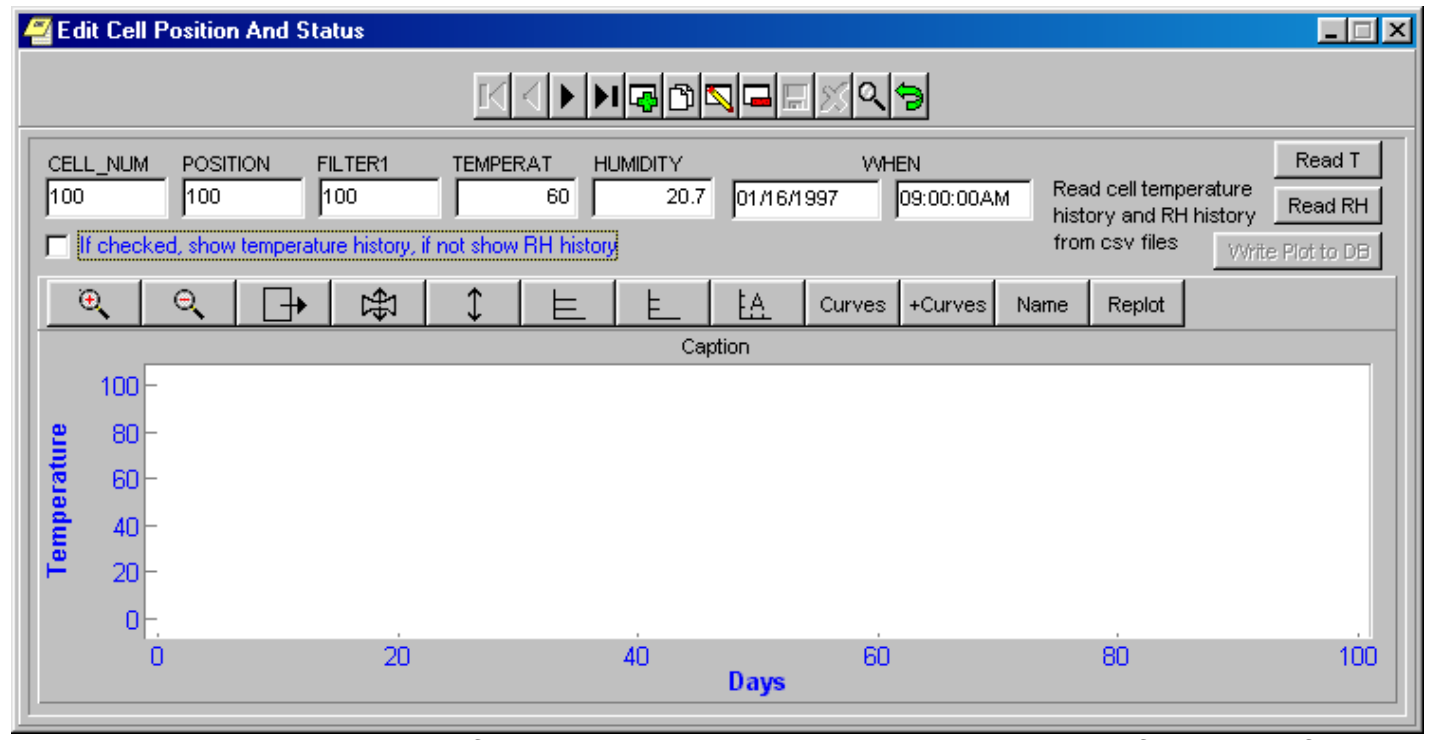

exposure of that cell. Provision was originally planned for up to 5 filters, but in practice only one filter has been used and there is no longer capability in the data-processing program for more than one filter. The temperature and humidity history of the cells can be included. The cell in the above table was placed in position 100 of the lamp field.

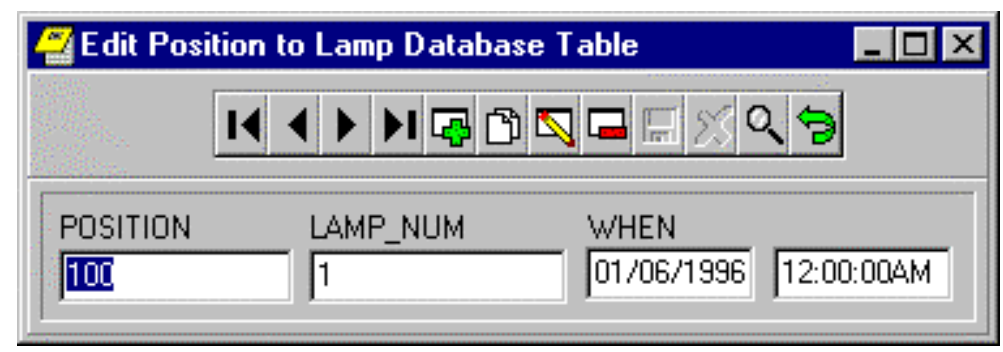

The cell will be placed in a position in the field illuminated by the lamp. The above table shows that position 100 was illuminated by lamp 1 at that time. 


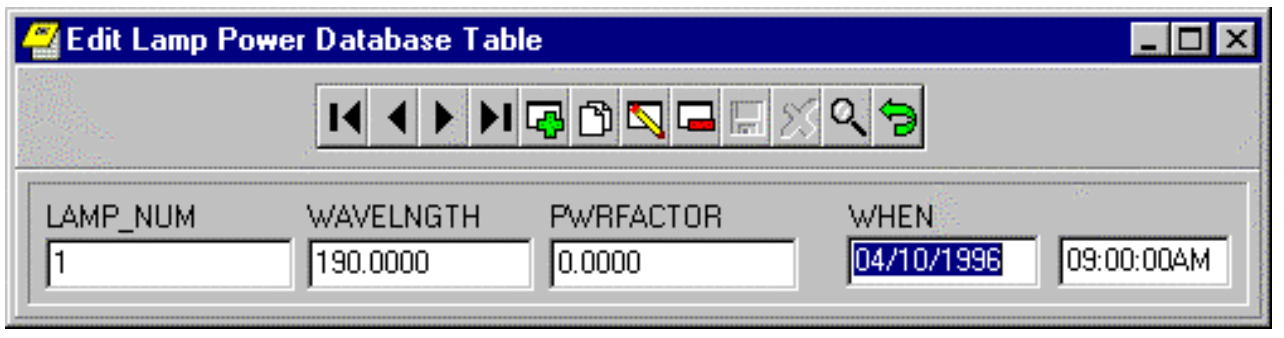

The UV-visible spectra currently cover the wavelength range 190 to 820 nanometers. A lamp power factor must be supplied for each of the wavelengths to convert the lamp intensity readings into watts. The lamp output factor at $190 \mathrm{~nm}$ is 0 . Non-zero lamp output begins at about 270 $\mathrm{nm}$.

\section{Dragging and dropping}

Where feasible, data entry has been facilitated by implementing dragging and dropping, shown here by dragging and dropping the supplier number. First, assign and show (for editing) the two windows in this case the Suppliers table and the Filter IDs table. Line up the two windows side by side. Position the mouse cursor over the SUPPLR_NUM edit box, left click and hold the mouse button down. When the mouse cursor is moved out of the box, the mouse cursor should change to the "forbidden" symbol. Note: a drag can not begin if the edit box is already selected for keyboard input. In that case, click in one of the other boxes to move the input focus and then begin the drag from the original box.

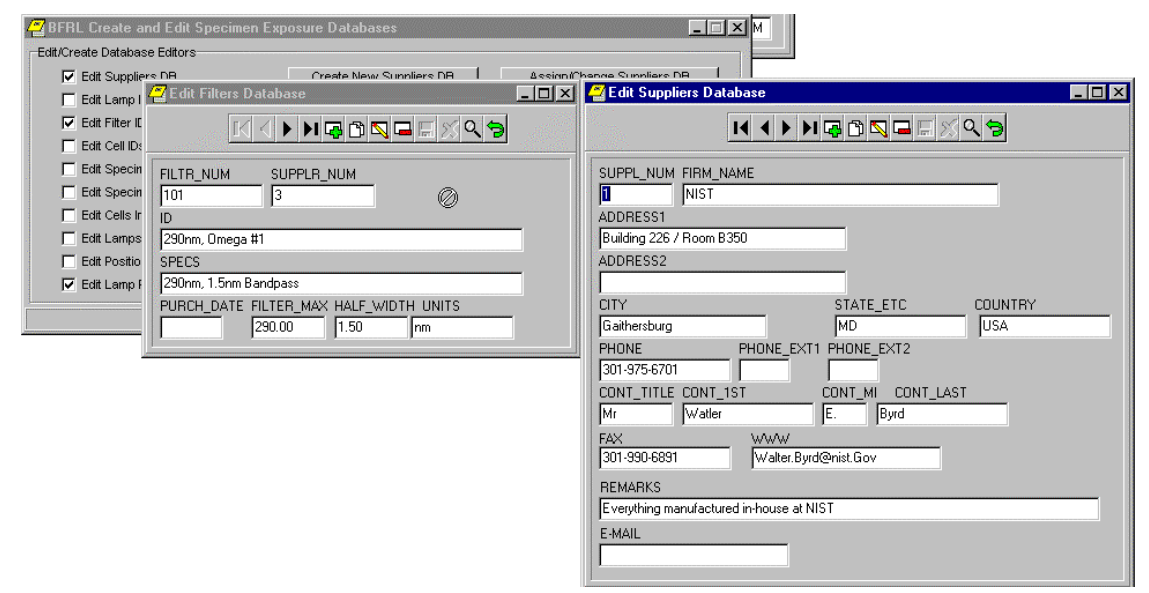

When the mouse cursor goes over a destination which is set to accept a drop, the cursor changes, as shown below. Releasing the mouse button will complete the drag and drop and, if all went well, the supplier number will appear in the filter IDs dialog box. 


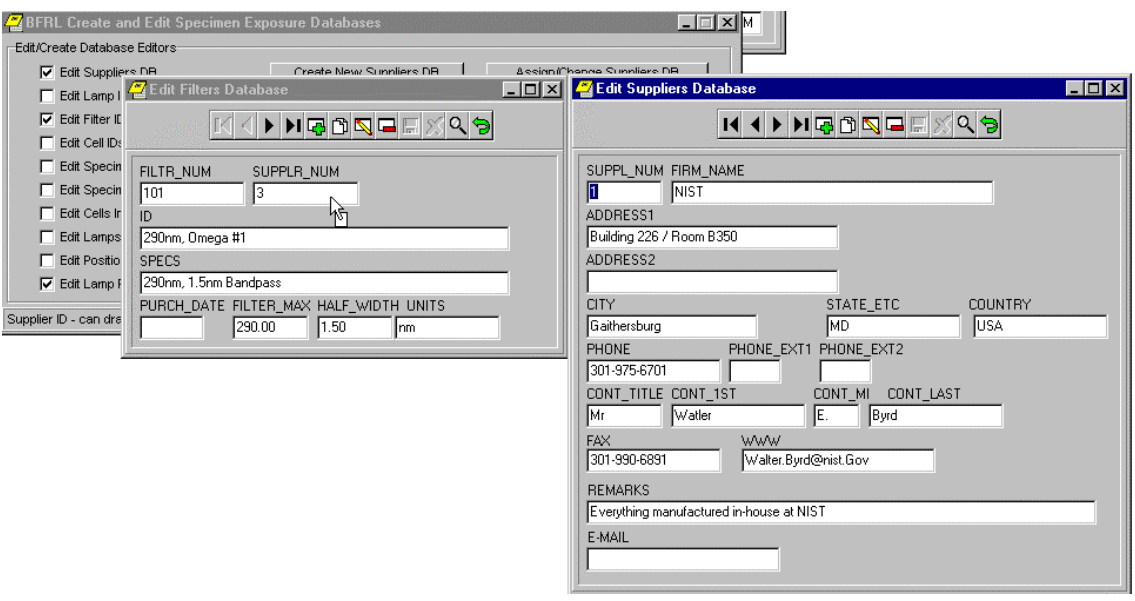

\section{Reading In Spectra}

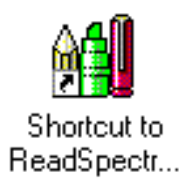

Spectra may be read in from csv files (see later) or from JCAMP files.

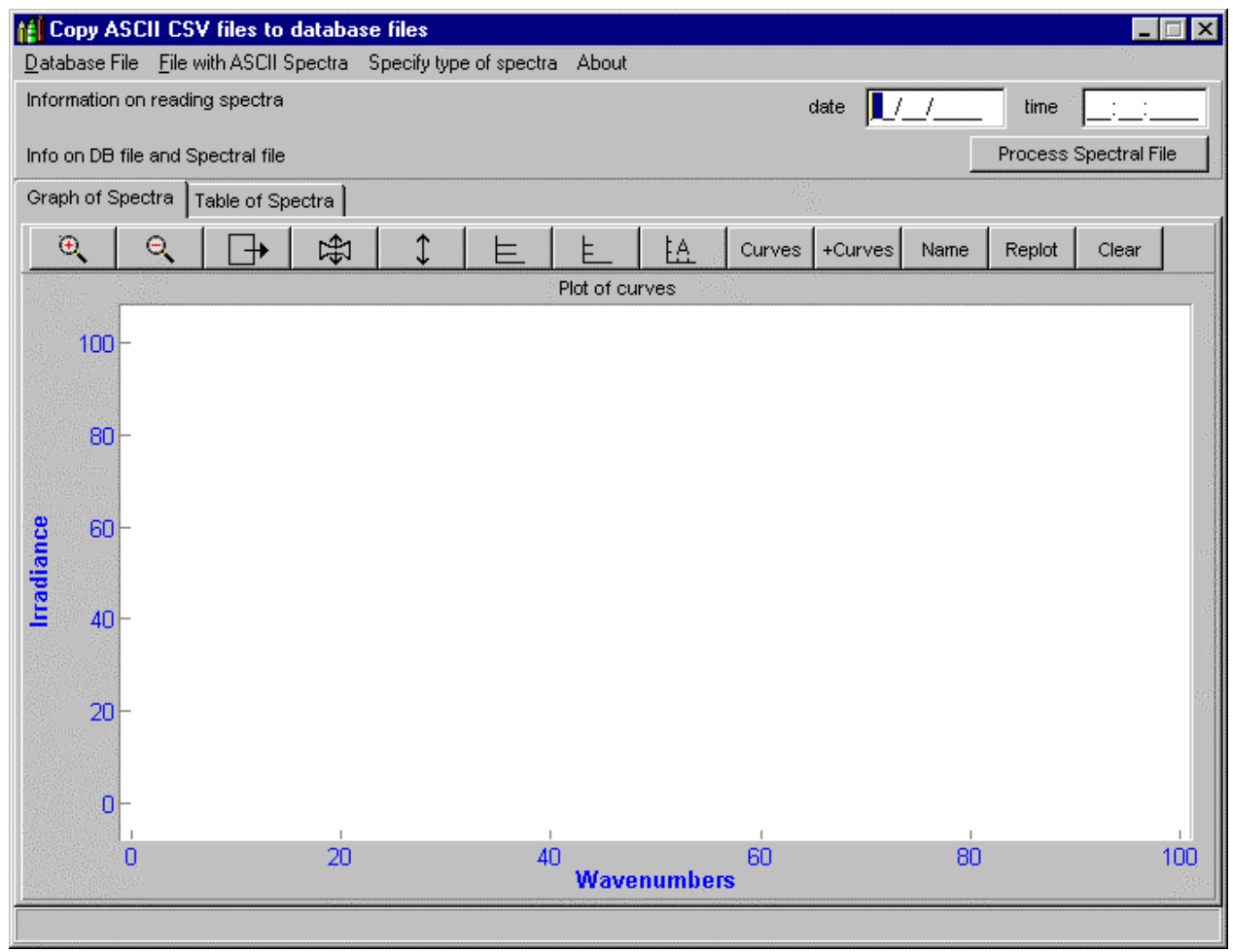

The first step is to select an existing database table or create a new one using the Database File option:

menu item. Select the appropriate menu 


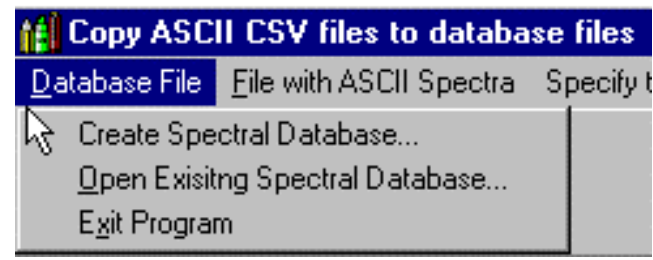

If a table is created, it remains assigned to the program. The first step is to create a new table or assign an existing table.

The second step is to tell the program where to find the information to be read in.

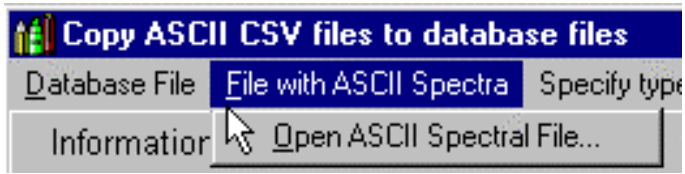

This file must be a CSV file, which is a text (ASCII) file in which the various items on a line are separated by some commonly used, easily recognizable character such as tab or space, hence the name and file extension C(ommon) S(eparator) V(alue). If the file contains the names of other files, those files will be read in one at a time as JCAMP files, spectral files prepared according to the format of the Joint Committee on Atomic and Molecular Parameters. Otherwise, the file is assumed to contain the wavelength as the first item on each line, followed by the $y$ values of the spectra - lamp irradiance, filter transmittance, or specimen UV-visible absorbance - in successive columns separated by spaces or tabs. The first line contains the lamp number, specimen number or filter number for the particular column. Examples of csv files are given later.

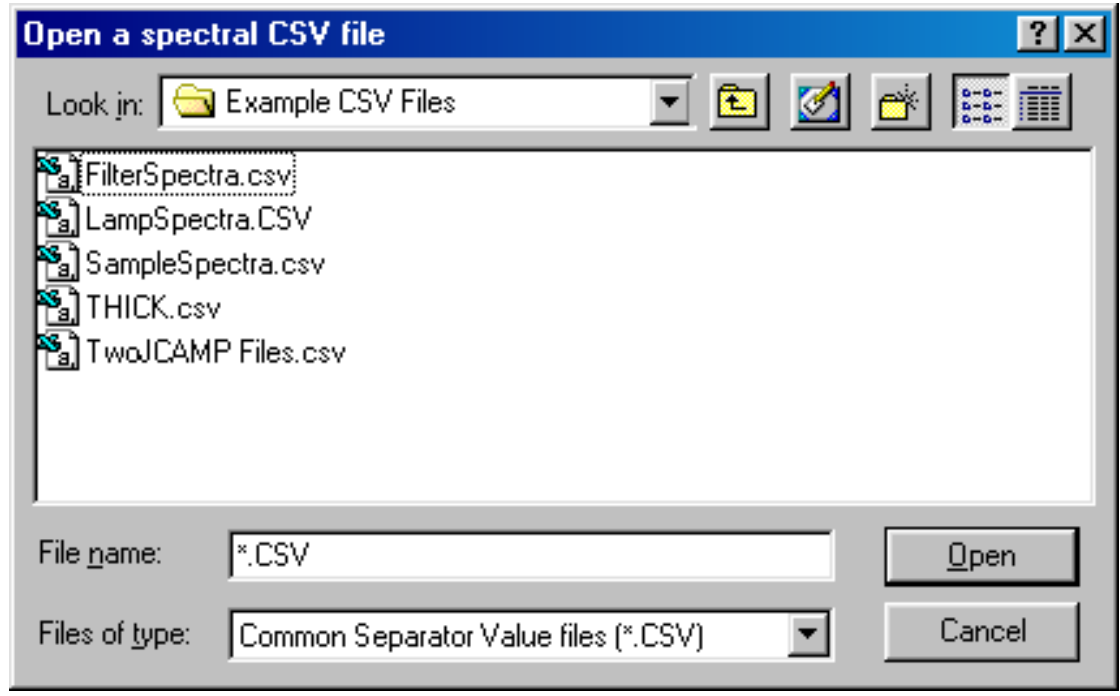

The programs can determine whether a database table is a table of spectra from the field names (headings) in the table. A spectrum database table contains a TYPE_NUM which tells the program whether 
the spectrum is for a lamp (TYPE_NUM =1), filter (TYPE_NUM =2), specimen UV (TYPE_NUM $=3$ ) or specimen IR (TYPE_NUM =4). This number is assigned by choosing the appropriate sub-menu option under the "Specify type of spectra" menu option.

\begin{tabular}{|c|c|}
\hline \multicolumn{2}{|c|}{ Afi Copy ASCII CSV files to database files } \\
\hline Database File File with ASCII Spectra & Specify type of spectra About \\
\hline Information on reading spectra & Lamp - from position in field \\
\hline Info on DB file and Spectral file & From Filter \\
\hline & UV from Specimen \\
\hline
\end{tabular}

The WHICH field in the table tells the program the lamp field position, the filter number, or the specimen number to which the spectrum belongs.

6iel Copy ASCII CSV files to database files
Database File File with ASCII Spectra Specify type of spectra
Information on reading spectra

The About menu option brings up a dialog box which identifies the program and provides some information on the configurations of Windows and the hardware.

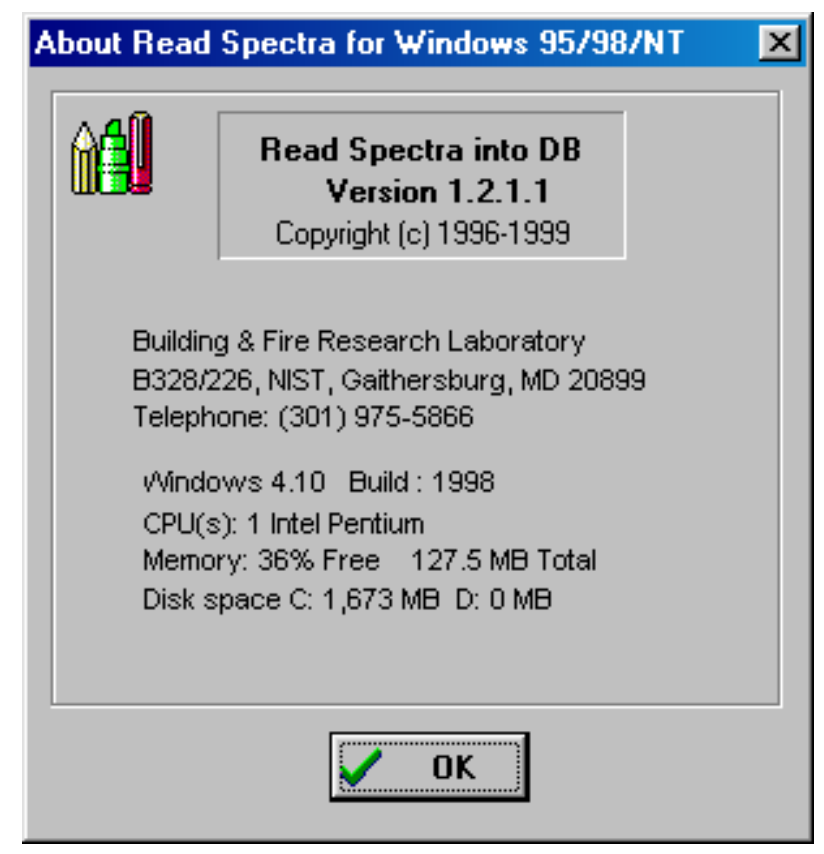

The date and time of the spectra to be read in (all of which will be given the same date and time) are provided in the date and time boxes.

Finally, the "Process Spectral File" button is clicked. 


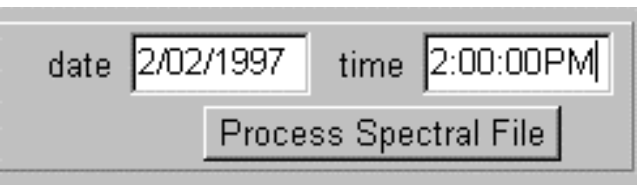

If the user forgot to provide the TYPE_NUM information on the type of spectrum, he is reminded, as shown below

Information

X

(i)

Need to specify an option - Lamp, Filter, or Specimen - from top menu

$$
\text { QKK }
$$

and provides the information as follows:

\begin{tabular}{|c|c|}
\hline \multicolumn{2}{|c|}{ Copy ASCII CSV files to database files } \\
\hline Database File File with ASCII Spectra & Specify type of spectra About \\
\hline Information on reading spectra & Lamp - from position in field \\
\hline Info on DB file and Spectral file & From Filter \\
\hline & UV from Specimen \\
\hline
\end{tabular}

Clicking again on the "Process Spectral File" button reads the spectra into the program and the program writes them to the database table. The program shows a plot of all the spectra which have been read in

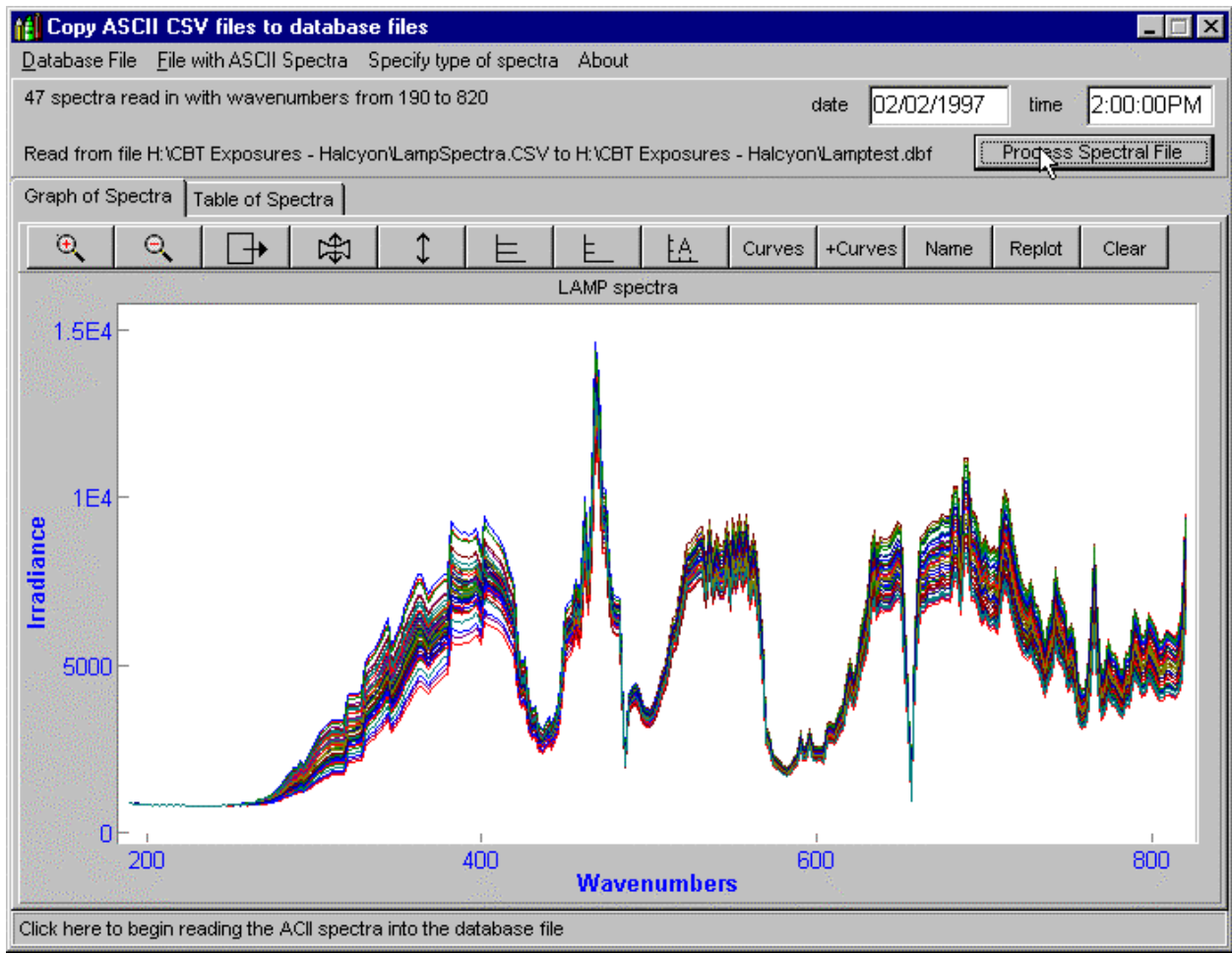


and also provides the numerical values in a grid. The first column is the wavelengths, the first row is the spectrum number. The body of the grid contains the $y$ values.

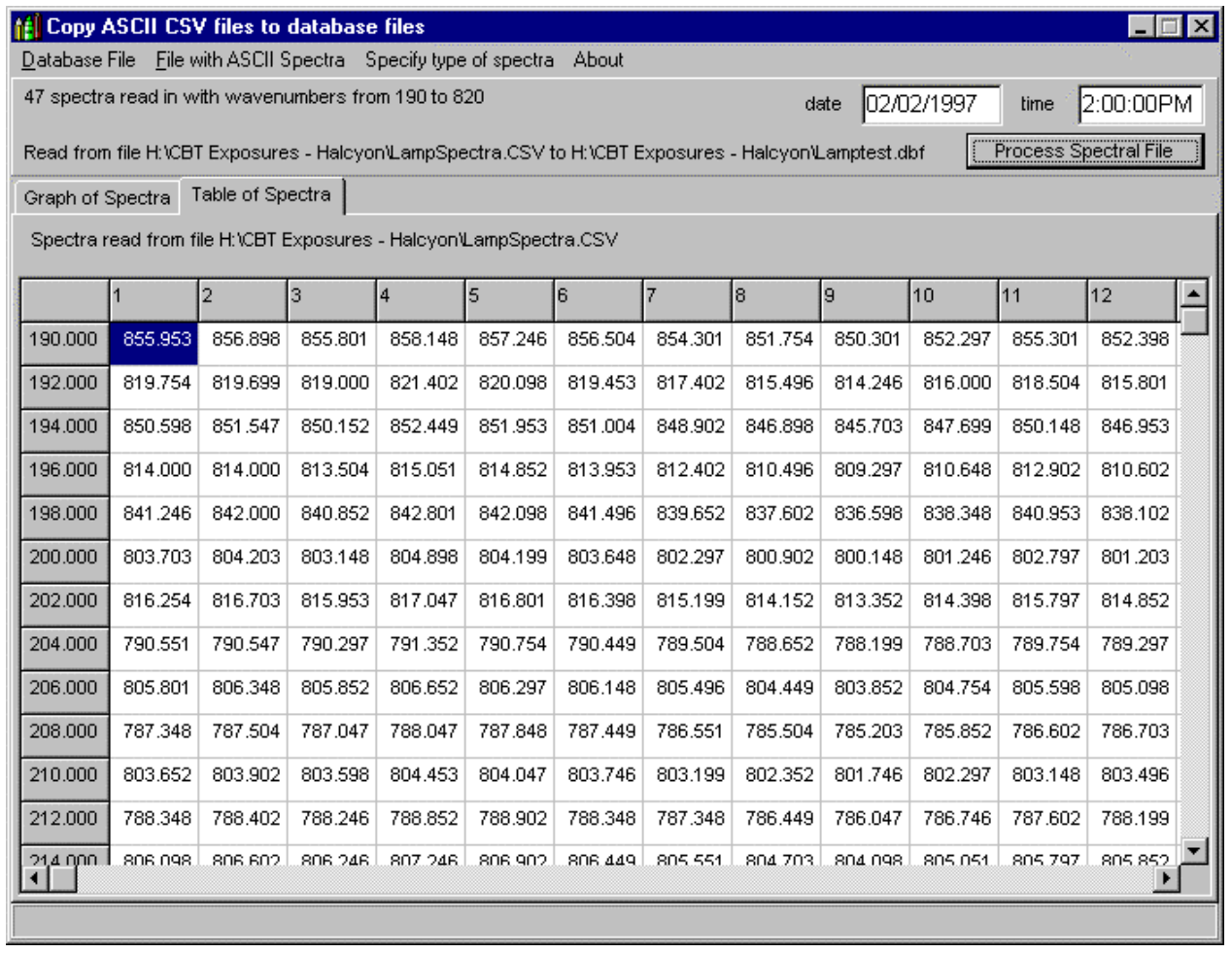

\section{Examples of the CSV files}

The first type of csv file is from an Excel spreadsheet file. The specimen number is across the top in row 1 and the wavelength of the spectral absorbance is in the first column, column A. The other columns contain the spectral absorbances for each specimen. This information would be exported as a CSV file so it could be read into the Read Spectra program.

\begin{tabular}{r|r|r|r|r|r|} 
& \multicolumn{1}{|c|}{$\mathbf{A}$} & \multicolumn{1}{c|}{$\mathbf{B}$} & \multicolumn{1}{c|}{ C } & \multicolumn{1}{c|}{ D } & \multicolumn{1}{c|}{ E } \\
\hline $\mathbf{1}$ & 0 & 100 & 101 & 102 & 103 \\
\hline $\mathbf{2}$ & 190 & 0.150223 & 0.134888 & 0.146042 & 0.150223 \\
\hline $\mathbf{3}$ & 192 & 0.162704 & 0.186646 & 0.180588 & 0.168625 \\
\hline $\mathbf{4}$ & 194 & 0.202927 & 0.187134 & 0.188263 & 0.191269 \\
\hline $\mathbf{5}$ & 196 & 0.232254 & 0.234833 & 0.243652 & 0.22673 \\
\hline $\mathbf{6}$ & 198 & 0.273895 & 0.270813 & 0.267151 & 0.278366 \\
\hline $\mathbf{7}$ & 200 & 0.359665 & 0.355133 & 0.361526 & 0.3508 \\
\hline $\mathbf{8}$ & $\mathbf{2 5}$ & 0.458801 & 0.449722 & 0.456192 & 0.462769 \\
\hline $\mathbf{9}$ & 204 & 0.546326 & 0.530685 & 0.530777 & 0.548355 \\
\hline $\mathbf{1 0}$ & 206 & 0.635666 & 0.630203 & 0.636948 & 0.634277
\end{tabular}


The second type of csv file is when IR spectra are to be read in as JCAMP files. In the two lines, below, from a file of that type, the spectrum for specimen 102 is in file B1-0-2.DX and the spectrum for specimen 108 is in B1-0-8.DX.

\section{2,B1-0-2.DX \\ 108,B1-0-8.DX}

JCAMP files are output by most spectrum-handling programs. An example of the beginning of a JCAMP file is:

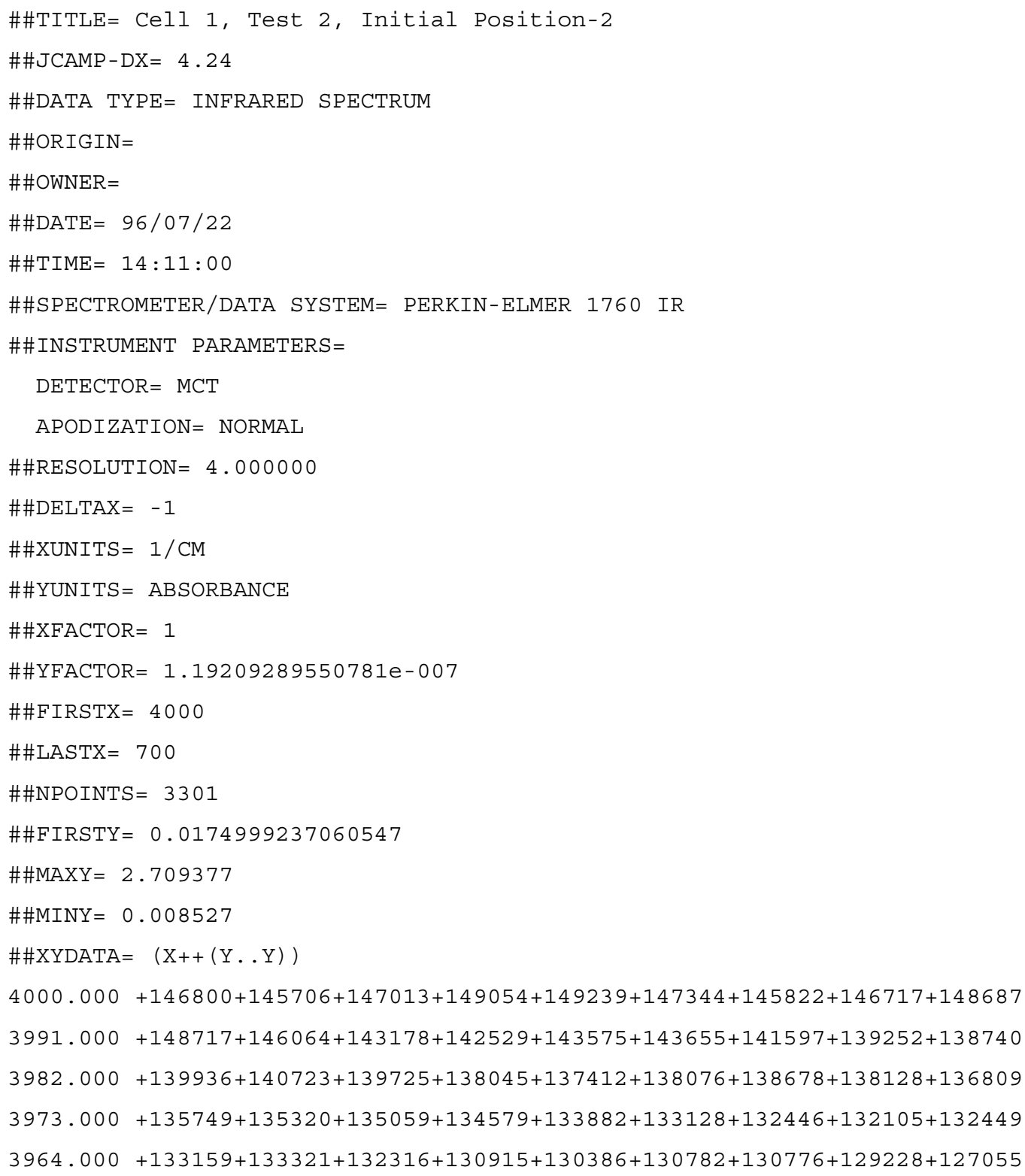


The spectra absorbances are provided as several absorbances per line, as in the last five lines of the example.

\section{Programs to examine and correct the contents of database tables}

Two such programs are provided. The LookAtTableGrids program allows the user to scroll through the records in a database table and to correct those records using the navigator bar. The spectra in a table may be examined, compared, and to some extent corrected using the LookAtSpectra program.

\section{LookAtTableGrids}

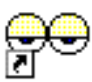

Shortcut to

LookAtT able..

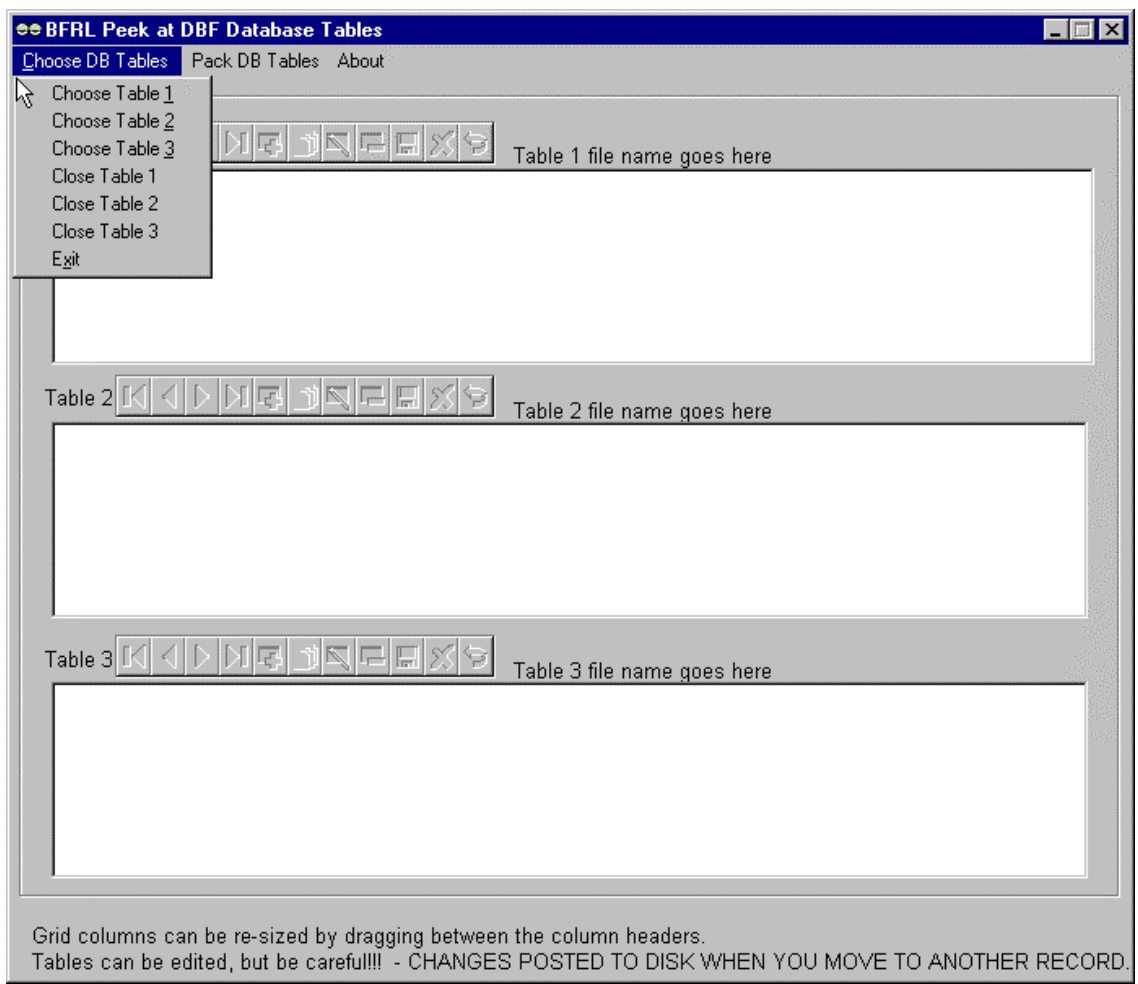

The database tables can be examined and edited, records can be deleted, and information can be retyped after setting up the editing or delete procedure using the navigator bar (over each table in the figure below). Tables can not be created in this program and editing using drag and drop can not be used. Up to three tables may be read into the program, as shown below. 


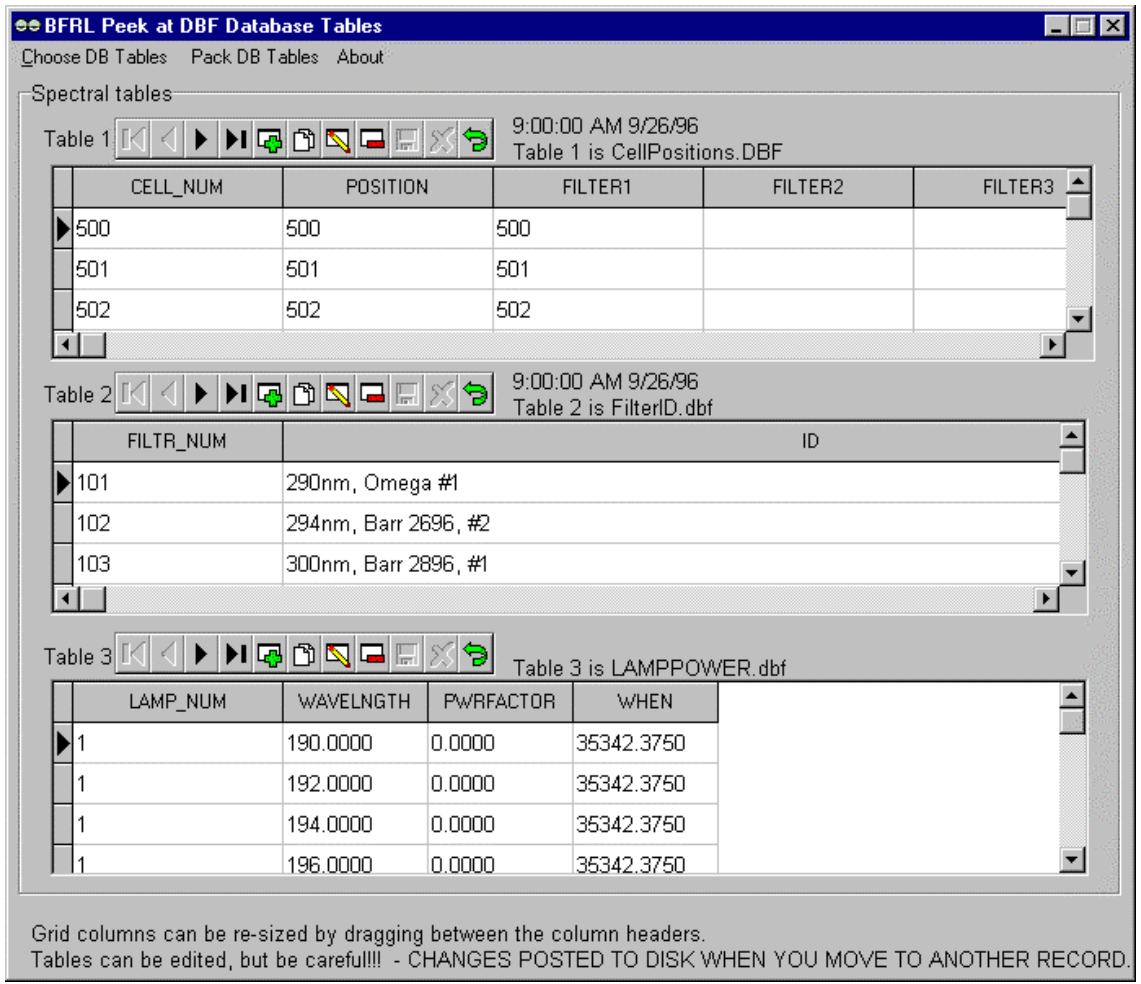

The columns may be resized by dragging the column heading separators.

\section{LookAtSpectra}

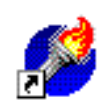

Shortcut to LookAtSpect...

The spectra in a table may be examined, corrected and compared using the LookAtSpectra program. Because the plot takes up most of the program window, spectral files can be only be examined one at a time. 


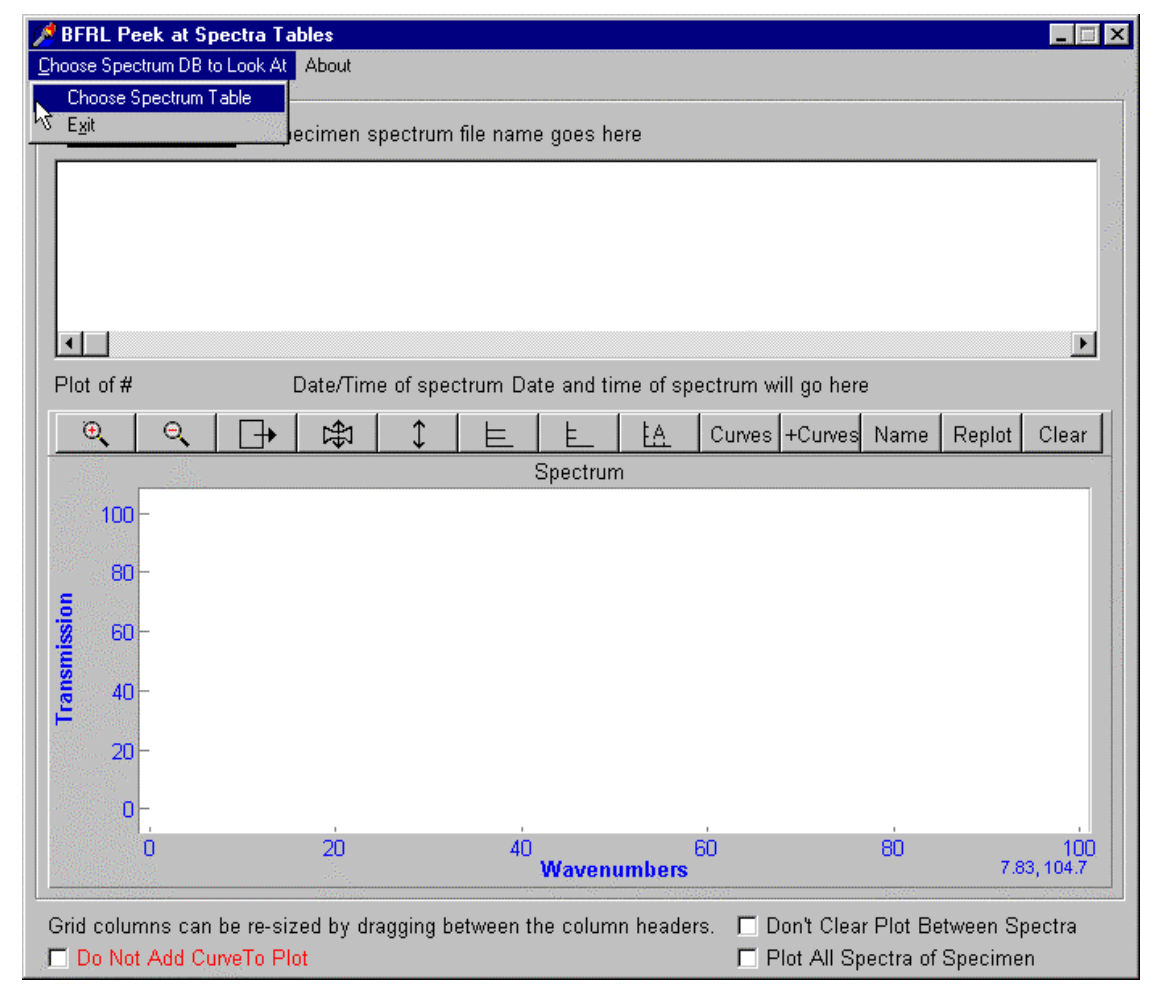

The file is read in using the "Choose Spectrum Table" menu item. Typed corrections can be made to the table parameters shown in the grid but not to the spectra.

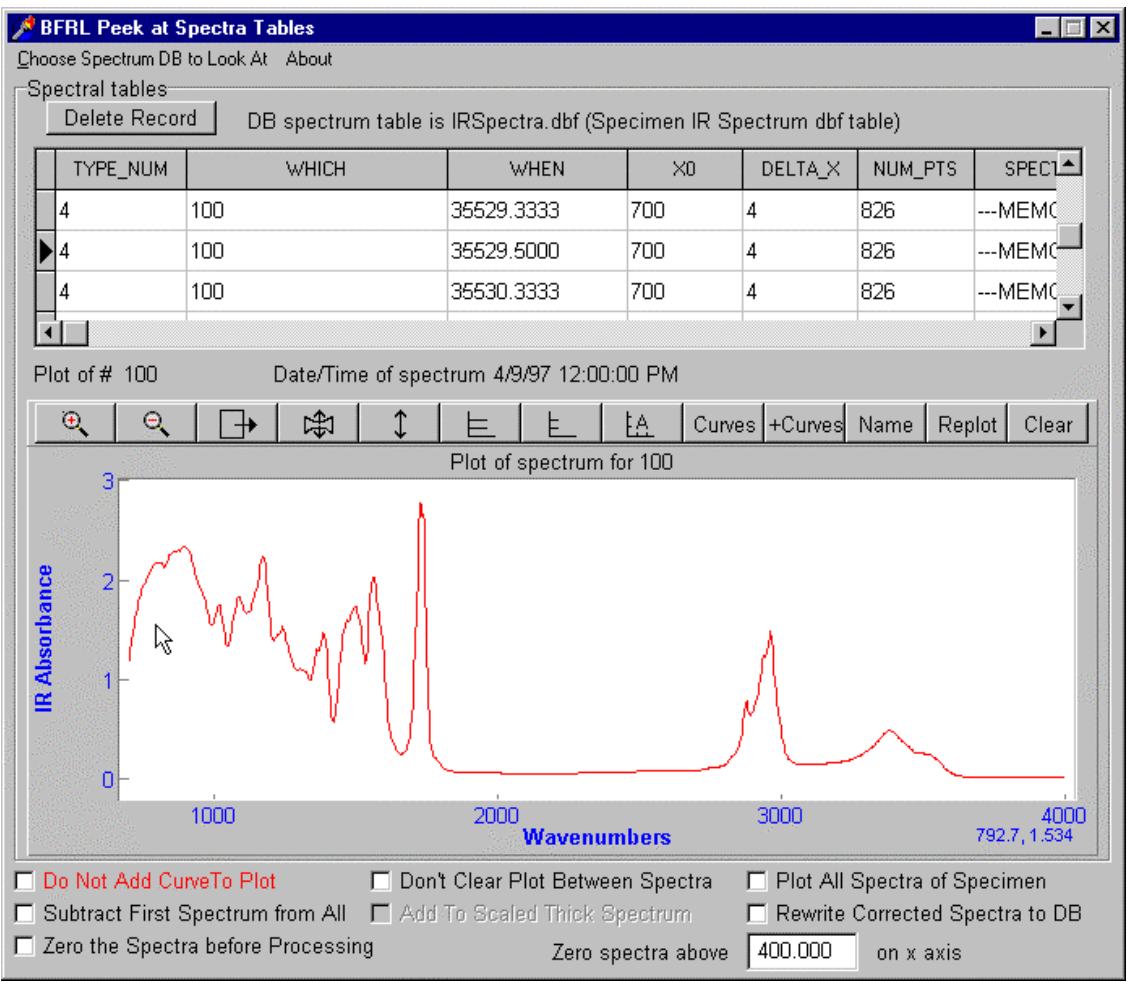

A record can be selected using the grid cursor at the left hand side of the grid or by clicking on the scroll bar at the right hand side of the grid. 
Records can be skipped by checking the "Do Not Add Curve to Plot" box.

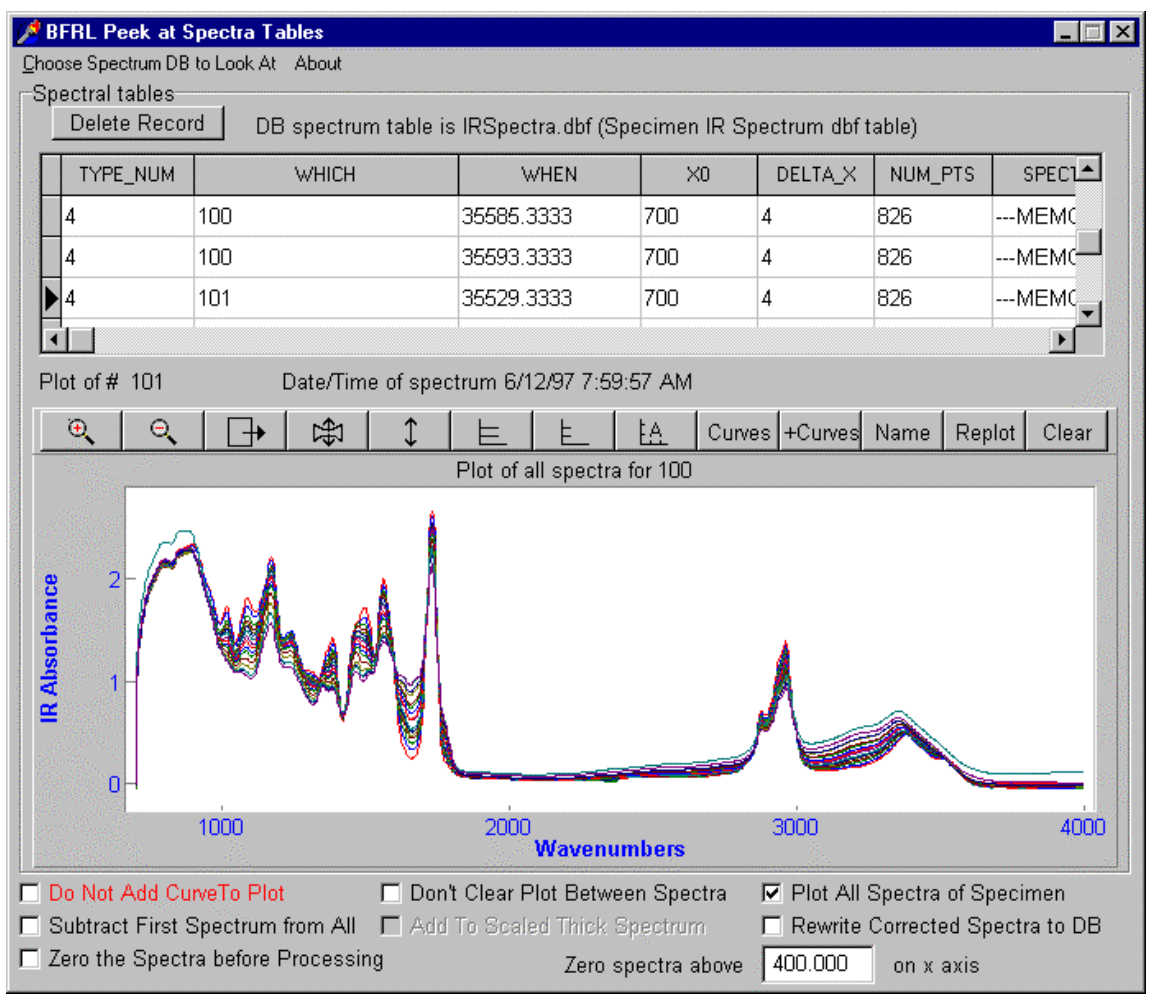

Spectra can be retained from record to record by checking the "Don't Clear Plot between Spectra" box.

If the "Plot All Spectra of Specimen" box is checked, all spectra for a new specimen (or lamp or filter) are added to the plot. This is a convenient way of viewing all the raw spectra for a specimen and of comparing specimen damages.

$\begin{array}{lll}\Gamma \text { Do Not Add CurveTo Plot } & \Gamma \text { Don't Clear Plot Between Spectra } & \bar{\nabla} \text { Plot All Spectra of Specimen } \\ \Gamma \text { Subtract First Spectrum from All } & \Gamma \text { Add To Scaled Thick Spectrum } & \Gamma \text { Rewrite Corrected Spectra to DB } \\ \Gamma \text { Zero the Spectra before Processing } & \text { Zero spectra above } & 400.000 \\ \end{array}$

Various other options were added to facilitate editing and correcting spectra, in particular the specimen UV spectra which had some sort of spectral contamination from the supporting plate. These options are shown in the above figure. "Do Not Add Curve To plot" allows the user to skip over spectra when navigating through the table. "Subtract First Spectrum from All" subtracts the first curve in the plot from each subsequent curve as the data are added to the plot. "Zero the Spectra before Processing" obeys the "Zero Spectra Above" number and is intended to clean up UV spectra which were not well enough measured above that point in the spectra. (If the UV spectrum of a thick specimen is used in scaled-down form, the data processing program will switch from the actual spectrum of the specimen to the scaled-down spectrum when the scaled-down spectrum has a higher absorbance than the original spectrum does. This allows for the appearance of degradation 
products in the original spectrum and yet overrides the original spectrum in regions where it clearly is not trustworthy.)

Corrected spectra may be immediately written to the database table by checking the "Rewrite Corrected Spectra to DB" check box.

\section{Data-processing program}

\section{Calculating dosage and damage in the specimen - AbsorbedUVvsIR.exe}

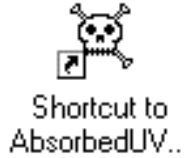

Shortcut to AbsorbedUV..

This program estimates the dose the specimen absorbs and relates the damage suffered by the specimen to this dosage.

\section{Startup}

Because start-up takes a few seconds, a "splash" screen is shown while the program is loading to show the user that something is happening.

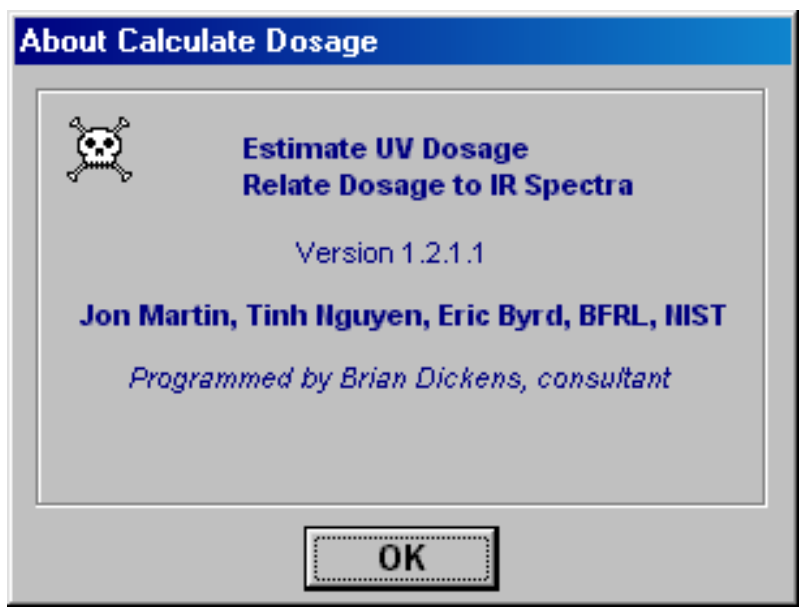




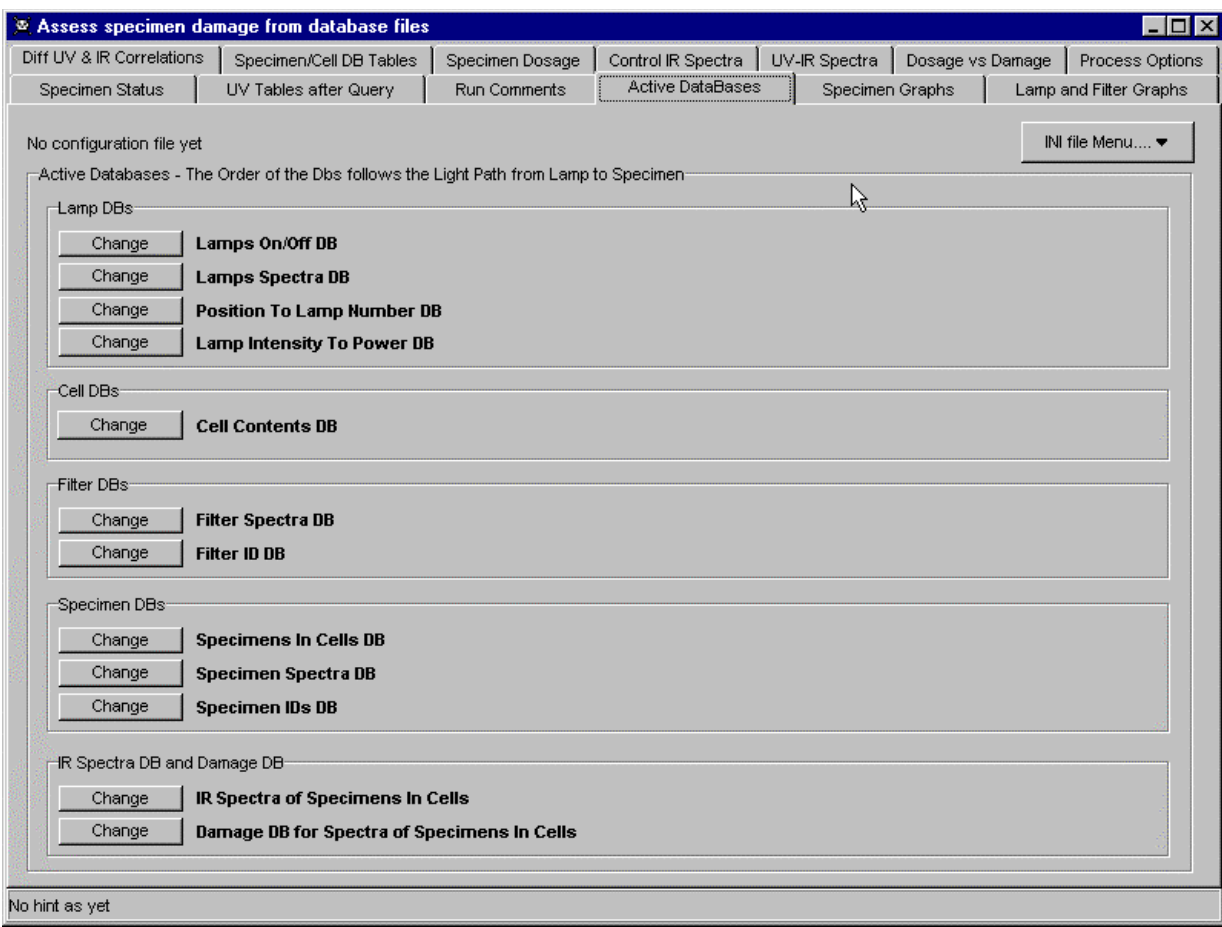

The program is divided into a series of pages, as shown below.

\begin{tabular}{|l|l|l|l|l|l|l|}
\hline Diff UV \& IR Correlations & SpecimeniCell DE Tables & Specimen Dosage & Control IR Spectra & UV-IR Spectra & Dosage vs Damage & Process Damage
\end{tabular}

Specimen Status | UV Tables after Query | Run Comments | Active DataBases | Specimen Graphs | Lamp and Fitter Graphs

\section{Specifying sets of database tables}

When the program ends, it optionally writes its current set of database tables and the parameters on the "Process Damage" Page to a UVExpStartUp.ini initialization file. Previously saved initialization files can be read in using the INI file menu, which is brought up by clicking on the "INI file Menu" button on the "Active Databases" page.

First select the Active Databases page

\begin{tabular}{|c|c|c|c|c|c|c|}
\hline \hline Diff UV \& IR Correlations & SpecineniCell DE Tables & Specimen Dosage & Control IR Spectra & UV-IR Spectra & Dosage vs Damage & Process Options \\
Specinen Status & UV Tables after Query & Run Comments & Active DataBases & Specinen Graphs & Lamp and Filter Graphs \\
\hline
\end{tabular}

then click on

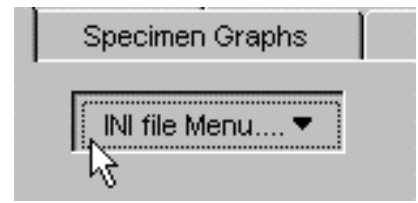

The menu which appears contains the following items: 


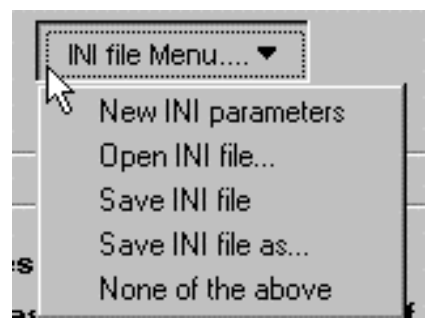

with the meanings:

\section{NEW}

clear the database table page, remove all tables from memory

\section{OPEN INI FILE...}

read in a new set of database tables (for an example of an INI file, see below) and "Process Damage" page parameters

\section{SAVE INI FILE}

save the current set of database tables and "Process Damage" page parameters in the current INI file

\section{SAVE INI FILE AS...}

save the current set of database tables and "Process Damage" page parameters in a new current INI file

\section{NONE OF THE ABOVE}

provides an escape route if nothing is to be done

Below is the file open dialog which appears if the user wants to read in a new set of database tables from a previously-saved INI file. 


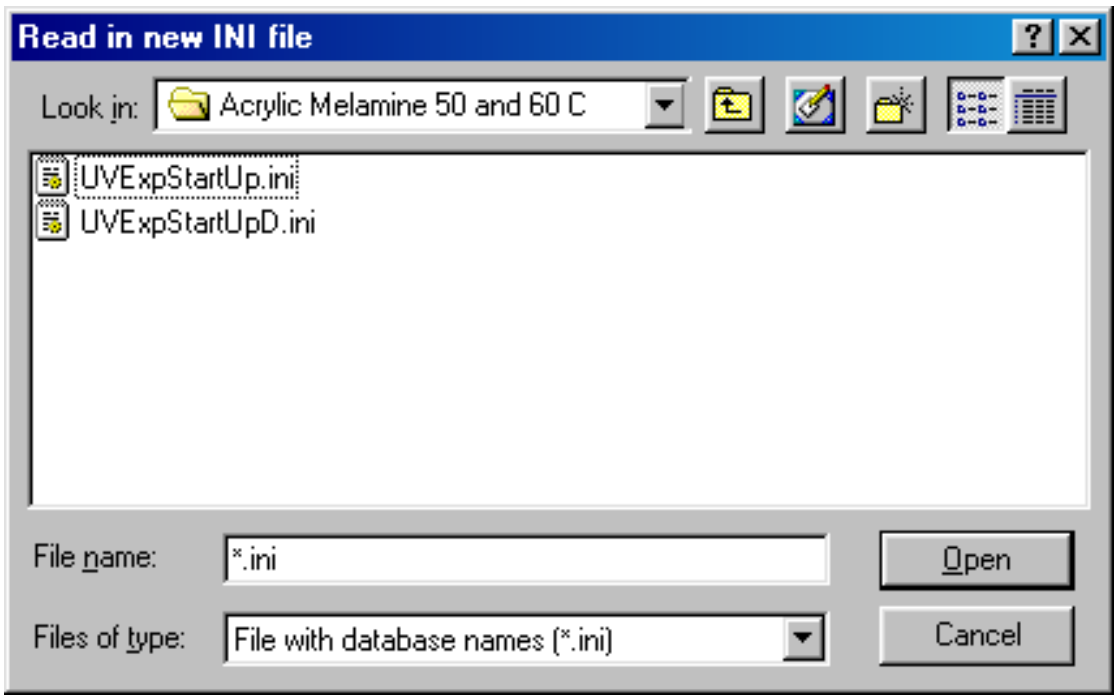

\section{Active Database Tables}

When the program begins, it does not have any database tables assigned to it. These tables can be assigned in three ways.

\section{READING A PREVIOUSLY WRITTEN INI FILE}

Use the

IN file Menu.... $\nabla$

button to go to the Open INI file menu, described below, then read in the file.

\section{SPECIFYING AND READING EACH DATABASE TABLE FILE IN TURN}

Click on the button marked "Change" corresponding to that table. This brings up a standard file open dialog box. Each database table file has the extension dbf.

\section{DRAGGING AND DROPPING FILES FROM WINDOWS EXPLORER}

To drag the file onto the "Change" button using Windows Explorer, move to the correct directory in Explorer and select the desired file by clicking on it with the left mouse button. While holding the mouse down, drag the mouse cursor over to the change button and release the left mouse button while the mouse cursor is over the change button. Drag only the dbf files, not the fpt or cdx files.

First, select the file: 


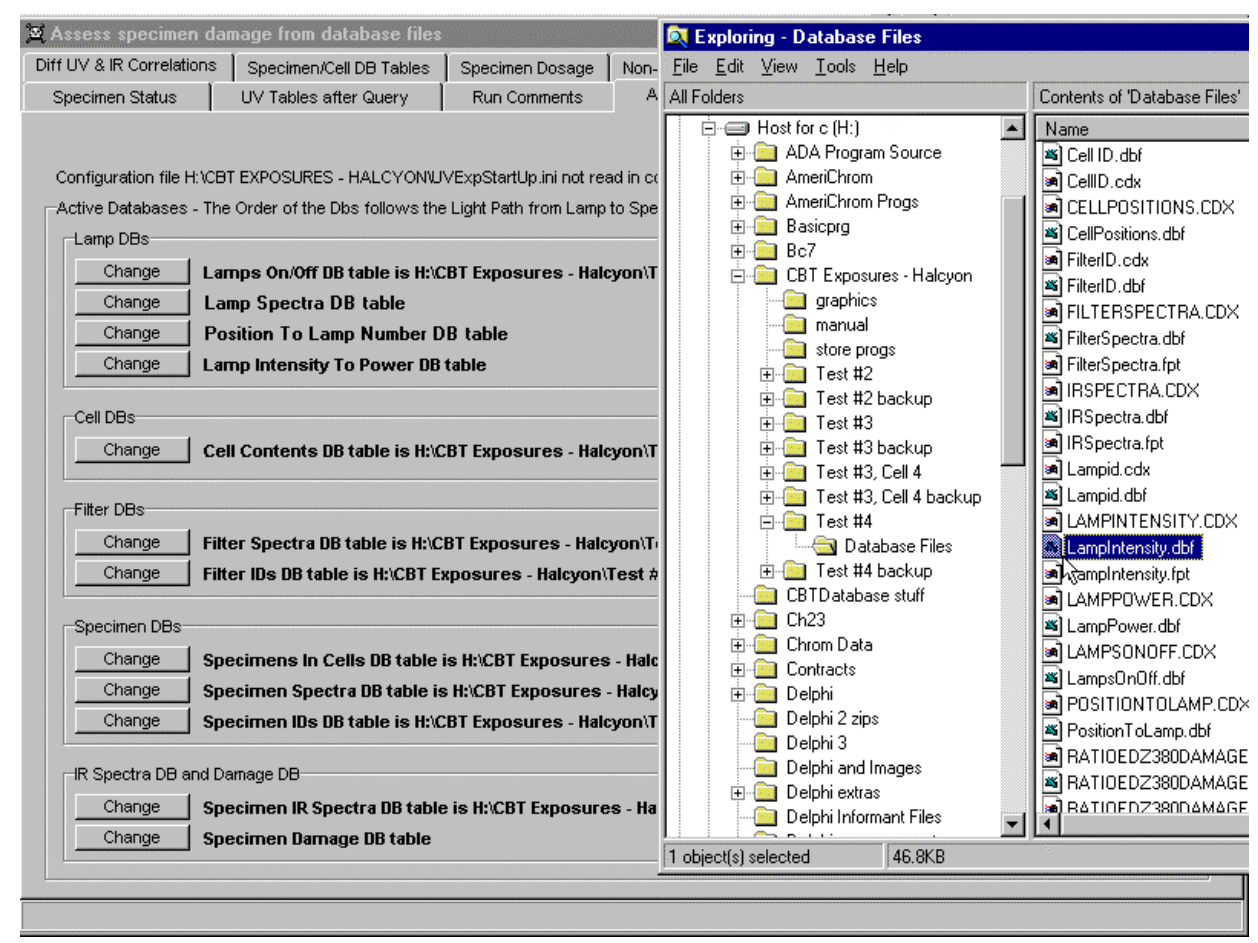

With the left mouse button held down, drag the mouse. The change in mouse cursor shows that dragging has begun.

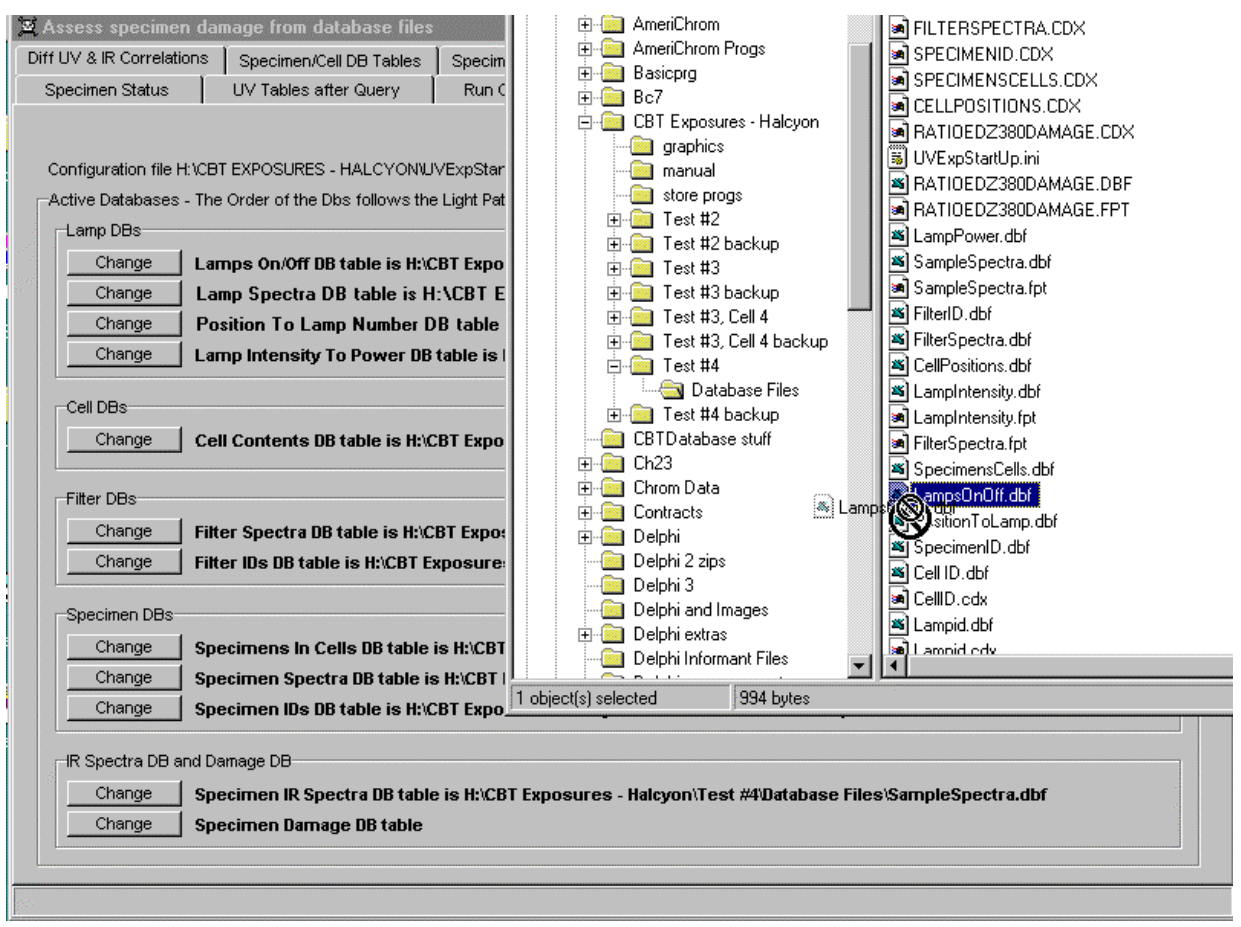

While the mouse cursor is over regions of the screen which will not accept the drop, the cursor displays a "forbidden" symbol. 


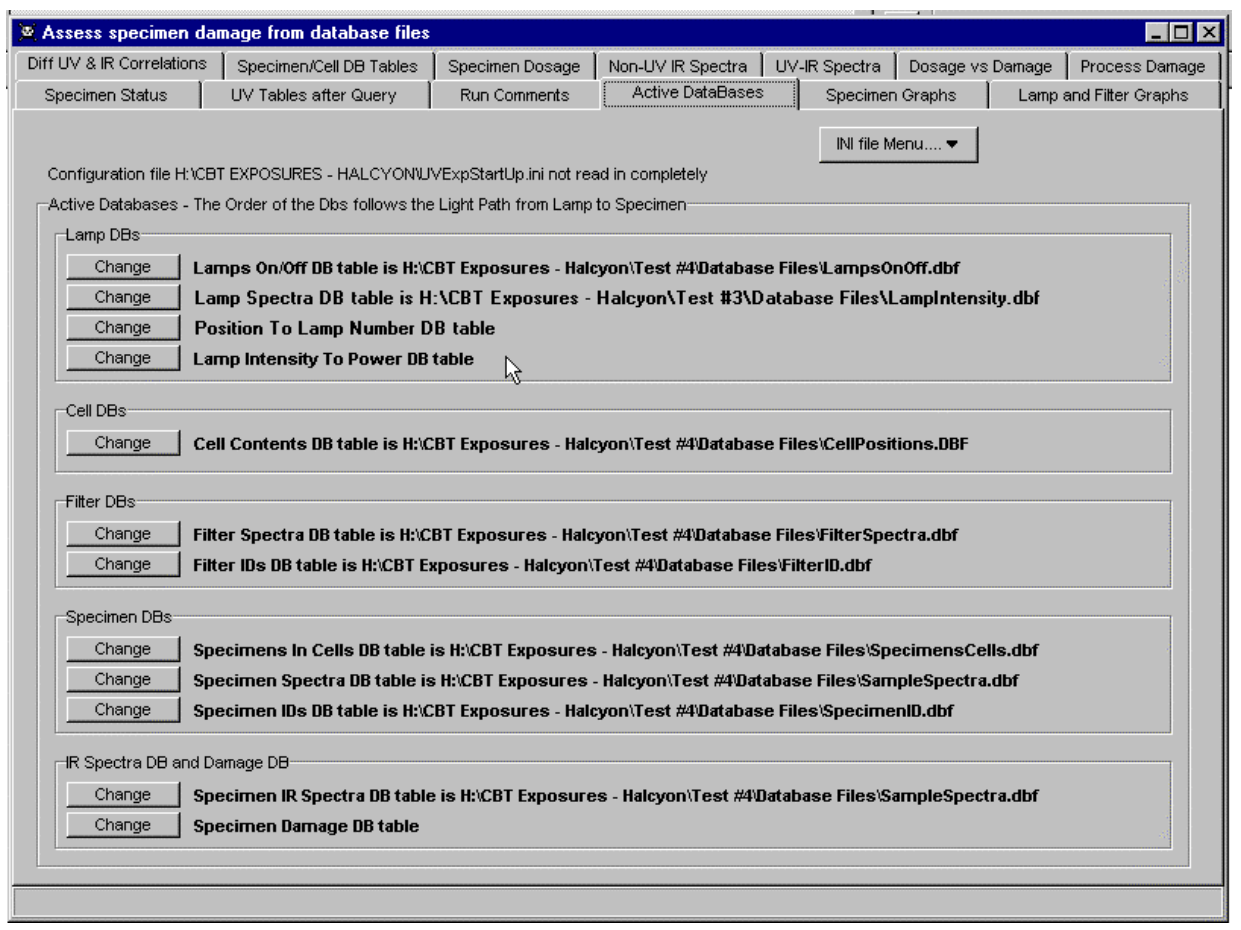

The dragged file has now been dropped on the "Change" button and the database table has been read in successfully.

\section{Checks on file identity}

Various checks are made on the database tables as they are read in. Below is an example where the data in the Lamp Intensity (lamp spectra) database table showed that the table being read in as a Lamp Intensity table was not set up to be a Lamp Intensity table.

\section{Error}

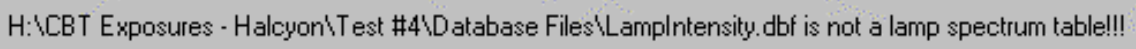

Clicking on OK or pressing the enter key (since the OK key is outlined and is therefore the default key which receives any key presses), brings up the following window.

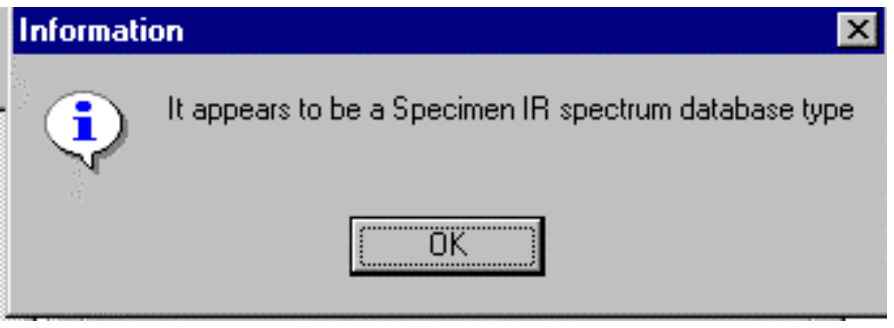

The program has been able to discern from the field names in the table that the table is a spectrum database table but, because the 
TYPE_NUM is 4 instead of 1, knows that the spectra are supposed to be specimen IR spectra, not lamp spectra. In this real-life example, the user had read lamp spectra into the database table but had forgotten to set the spectrum type to lamp instead of specimen IR. The program will not accept this table as a lamp spectrum table, even though the table really does contain lamp spectra, until the TYPE_NUM is changed from 4 to 1 . The checks are in place to protect the user (and the programmer) from making mistakes.

The currently active database tables are shown on this page of the program. All tables except the damage table must be present before the program can begin computations. They contain information that the program can only get from the database tables. The damage table can be created as needed because the information it contains is generated by the program.

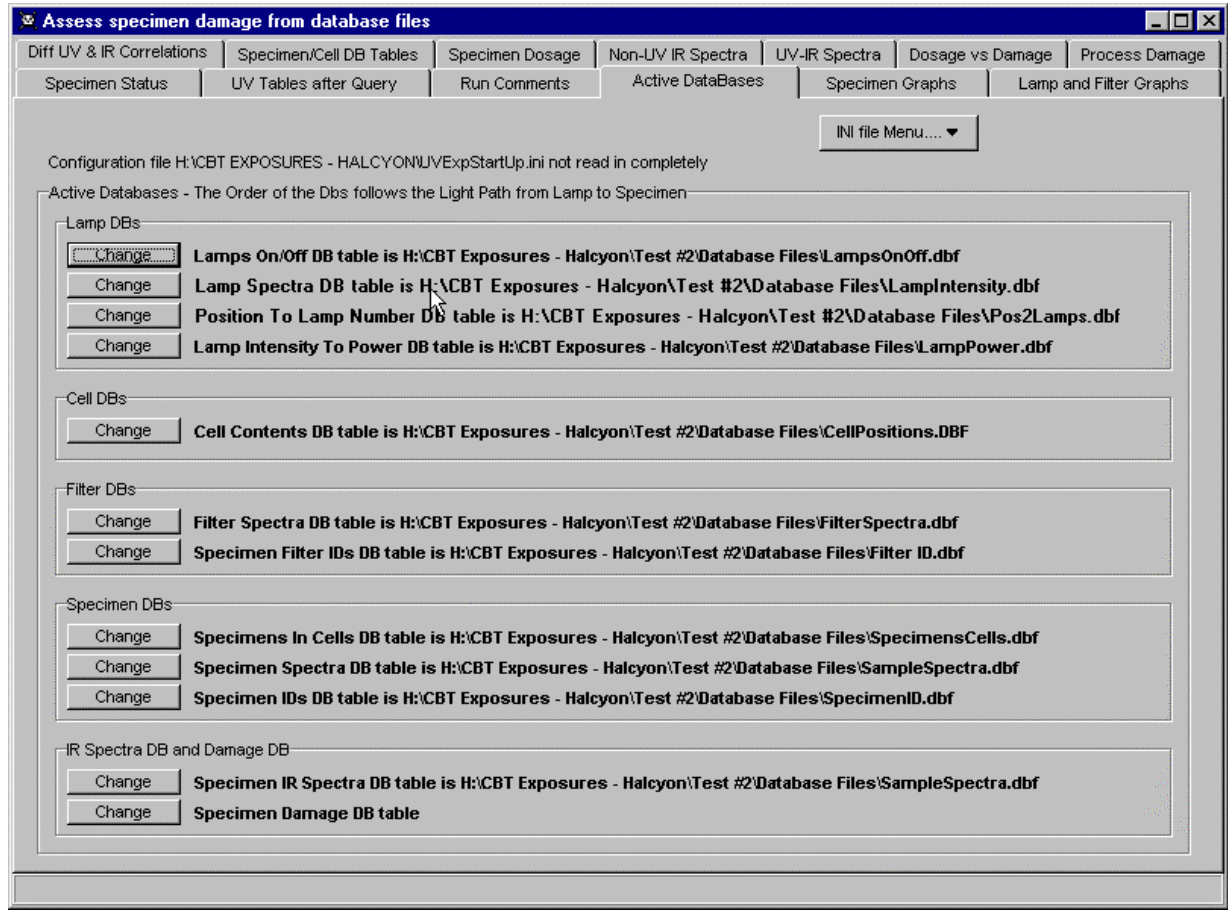

\section{Help Message Panel}

Many of the programs provide help messages on the functions of the controls in a help message bar at the bottom of the screen.

For example, when the cursor is positioned over the

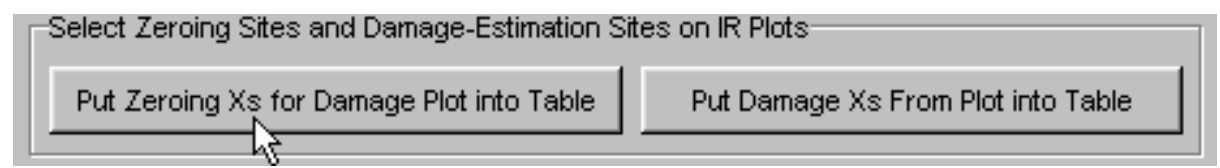


"Process Damage" page button which brings up the table of places at which to zero the IR spectra, the help message at the bottom of the screen is

Clicks on the IR spectra plots will be interpreted as sites at which to zero the IR spectra before manipulating them

\section{Control Parameters}

The program is very flexible, but this means that choices have to be provided and made. Most of the choices are on the "Process Damage", the last page of the tabbed page control. The choices will now be illustrated.

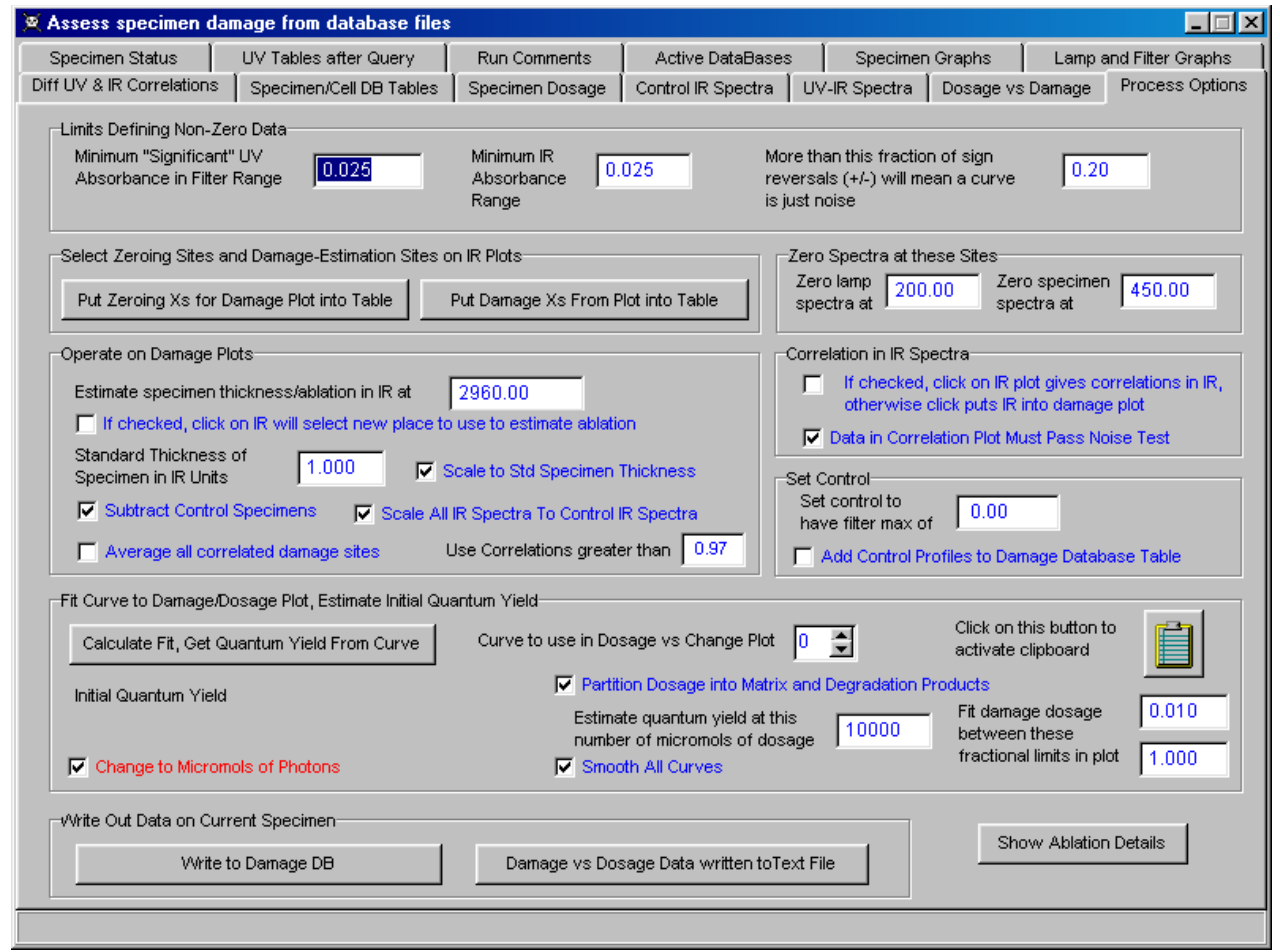

\section{ZEROING THE UV SPECTRA}

Before any curve can be scaled, the data must be corrected for offset from the true zero position. In the case of the spectral curves in this program, the $x$ values are typically wavelengths and are well known, but it may be that the $y$ values (lamp intensity, specimen UV absorbance) are not in "finished" form. For example, the measurement of the lamp intensity includes a dark current which will be non-zero even when the lamp is off. When the lamp spectra are zeroed, they are moved along the vertical axis until they are zero at the wavelength specified in the "Zero lamp spectra at" box. The specimen spectra will be similarly be moved along the vertical axis until they are zero at the wavelength specified in the "Zero specimen spectra at" box. If the value in a box does NOT fall in the wavelength range of the spectrum, no zeroing will be done, which provides a convenient method of turning the zeroing off. 
The filter spectra are not zeroed. The lamp and specimen UV-visible spectra are corrected by simple vertical shifts of the data. In the case of the lamp spectra, this is sufficient to remove the dark current equally from all places in the lamp spectra. No corrections for sloping backgrounds are applied to the specimen UV-visible spectra.

\section{Zeroing the Damage (IR) SPectra}

The damage spectra (currently IR spectra) must also be zeroed. There are several places in a typical IR spectrum where the absorbance can be expected to be zero. These places which are selected by clicking on a plot of the damage spectra. The intersection of the wavelength where the plot was clicked and the absorbance on the curve defines a point which will be made zero. All points on a straight line between any adjacent clicked points will also be made zero. If there are no adjacent clicked points, as for example when the clicked point is the first or last clicked point in the plot, all points from the clicked point to the beginning or end of the plot will have the y coordinate of the clicked point subtracted from their $y$ coordinates. This means that a series of straight lines can be defined by clicking on the IR plot and the straight lines between these points will define the new zero baseline.

To be able to click sensibly on a plot, there must be at least one curve in the plot so the first thing to do is process the data for a specimen to put curves in the plot (without writing the derived values to the damage database table).

To specify where to zero the spectra, click on the "Put Zeroing Xs for Damage Plot into Table" button on the "Process Damage" page (with at least one curve in the IR spectra plots).

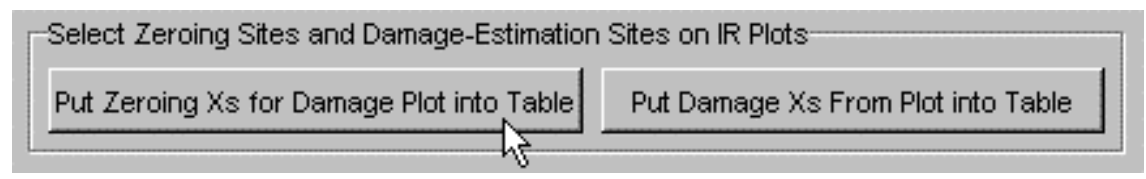

The table of sites at which to zero the damage plot is then shown and the damage plot is displayed (see the next figure). Sites can be typed into the table or put into the table by right-clicking on the damage plot. Note that the site produced by clicking is entered into the table of zeroing sites where the cursor is so, if the cursor is at the top of the table, successive clicks will move down the table, replacing whatever is already in the table. To avoid wiping out what is already in the table, click on an empty line in the table. When the process is ended, the table will be tidied up by the program.

Zeroing takes place only on the data after they have been read into the program. The original data in the database table are left untouched. 


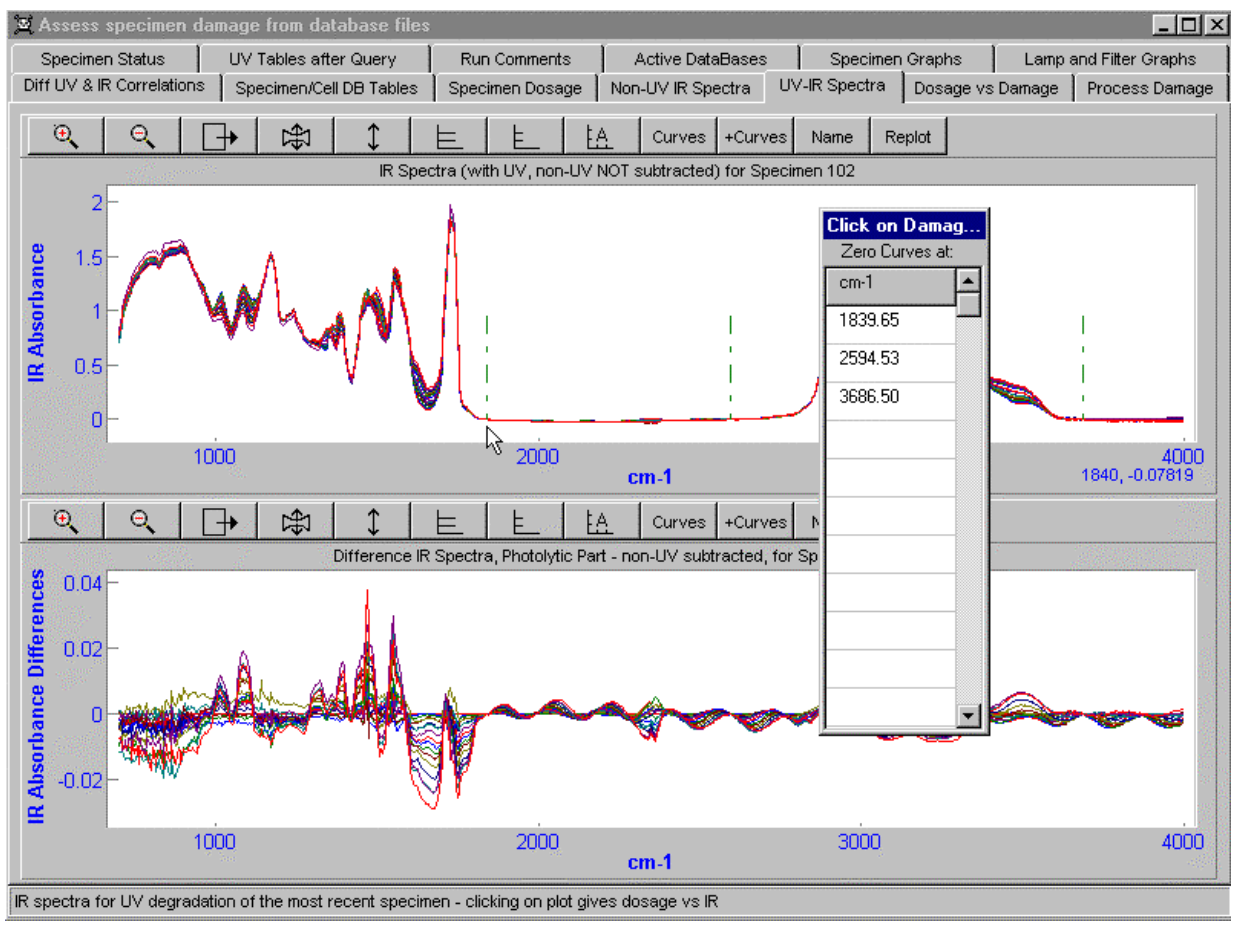

The process of adding zeroing sites to the table must be ended so that mouse clicks can revert to their normal usage. The "Put Zeroing Xs for Damage Plot into Table" button on the "Process Damage" page is down during the zero site adding process. Re-clicking on this button ends the process. The program will now use the list of sites to zero all damage spectra before they are processed further. It is essential to know the zero in spectra, or indeed in any curves, before the data are scaled or used to estimate dosage and damage.

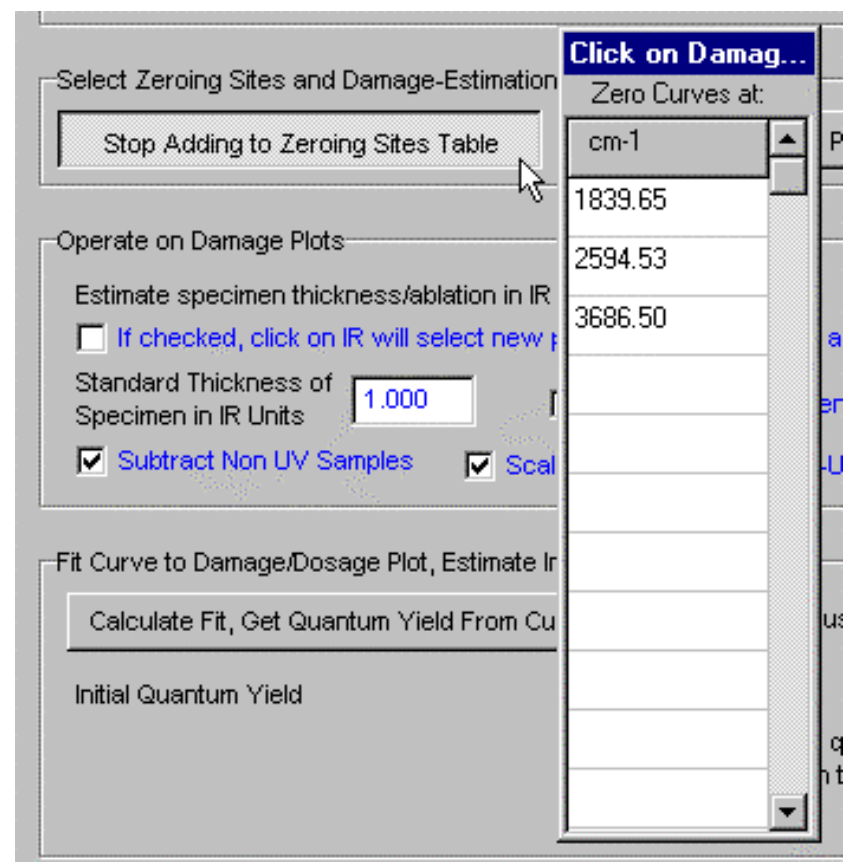




\section{Selecting the Damage Sites in the Damage Spectra}

The spectral sites at which damage is to be assessed are selected in a procedure similar to that used to selecting the zeroing sites. To specify where to monitor the damage spectra, click on the "Put Damage Xs from Plot into Table" button on the "Process Damage" page.

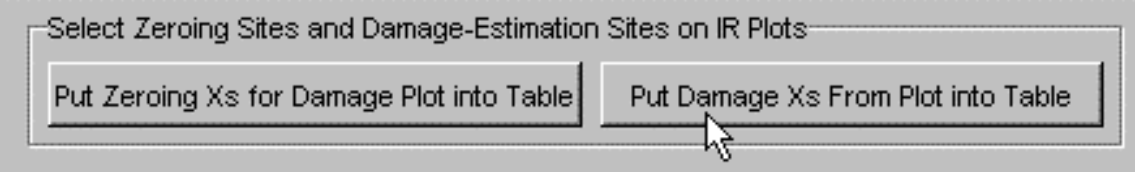

This brings up the Damage Site table. Right mouse clicks on the damage plot (or, better, on the difference damage plot) will put the abscissa values (wave number in the case of IR spectra) for these sites into the table. The entries begin where the cursor is in the table, so before clicking on the damage plots, put the cursor in an empty row of the table.

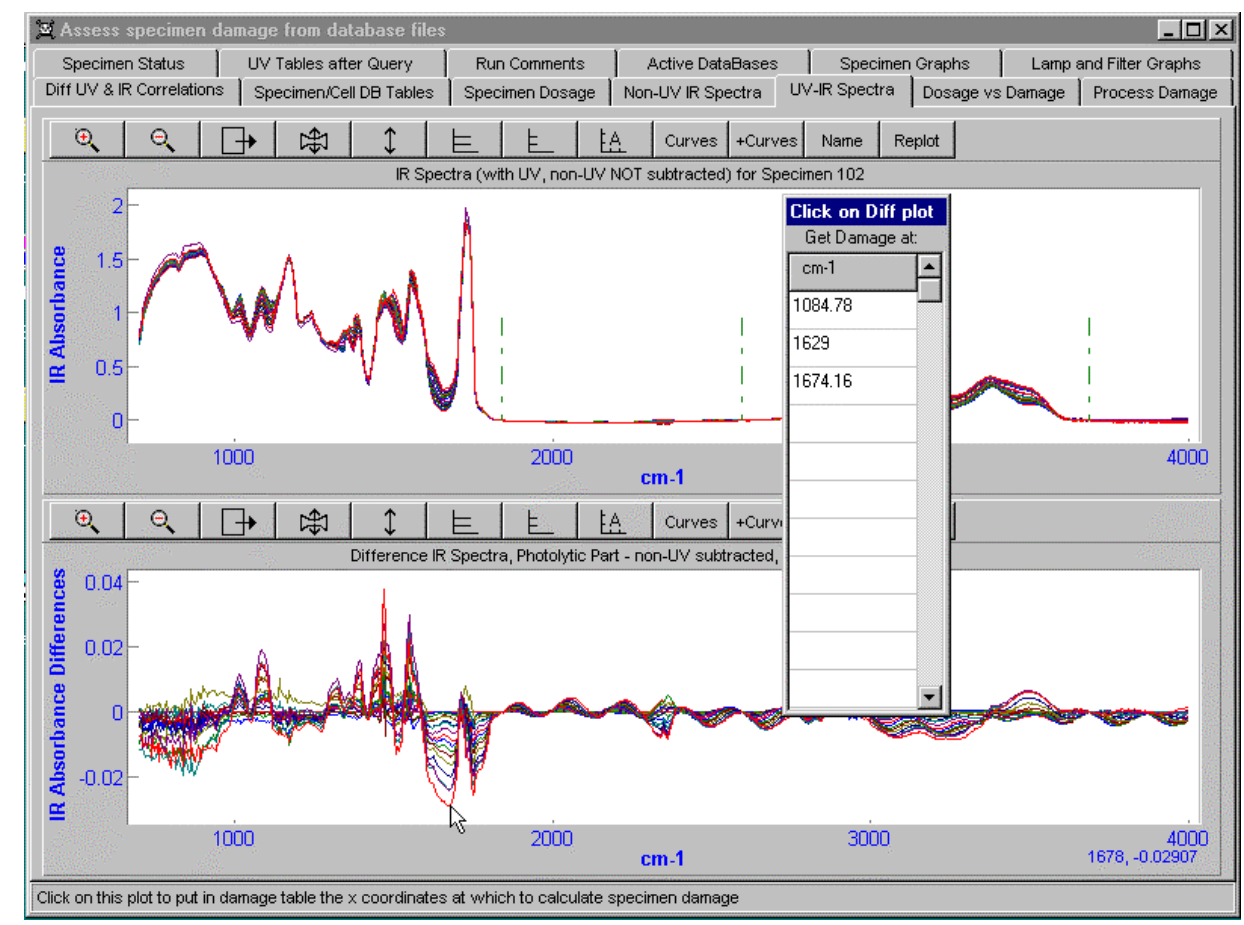

The process is ended by re-clicking on the "Put Damage Xs from Plot into Table" button on the "Process Damage" page. This button is kept down during the process to show that the process is underway. When the table is hidden by the program, the entries in the table are tidied up so that there are no empty rows. When a specimen is processed automatically, damage will estimated at the sites in this table. 


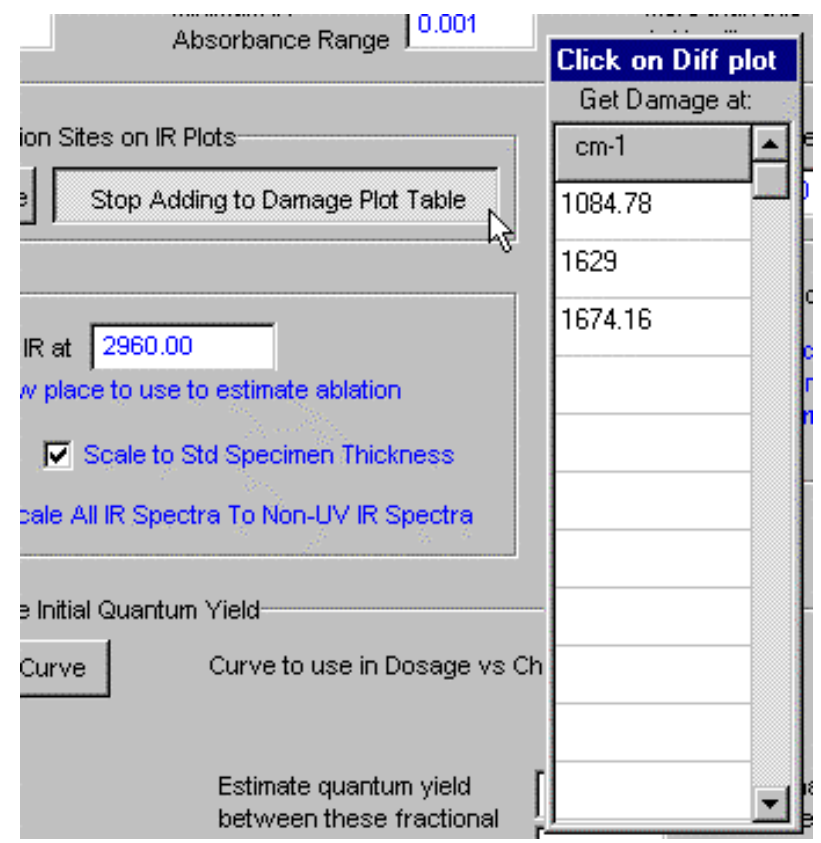

\section{AbLATION}

Some specimens suffer loss of material during the exposures. This ablation must be corrected if effects in specimens of different thicknesses, for example the controls and the exposed specimens, are to be subtracted. The program provides a mechanism for correcting for ablation by scaling the IR (damage) spectra so that the absorbance at a user-specified wavelength has a pre-specified value. Alternatively, the thickness of the specimen can be estimated by comparing its absorbance at some wavelength with the absorbance at the same wavelength of a specimen of known thickness. The wavelength in the damage spectrum at which to estimate specimen thickness should not be affected by photolytic or hydrolytic degradation - it will of course be affected by the loss of material as the film ablates. It is specified in the box next to the "Estimate specimen thickness/ablation at" label. This box can conveniently be filled in by first checking the check box labeled "If checked, click on IR will select new place to use to estimate ablation" then clicking on the IR plot. Uncheck the check box before proceeding.

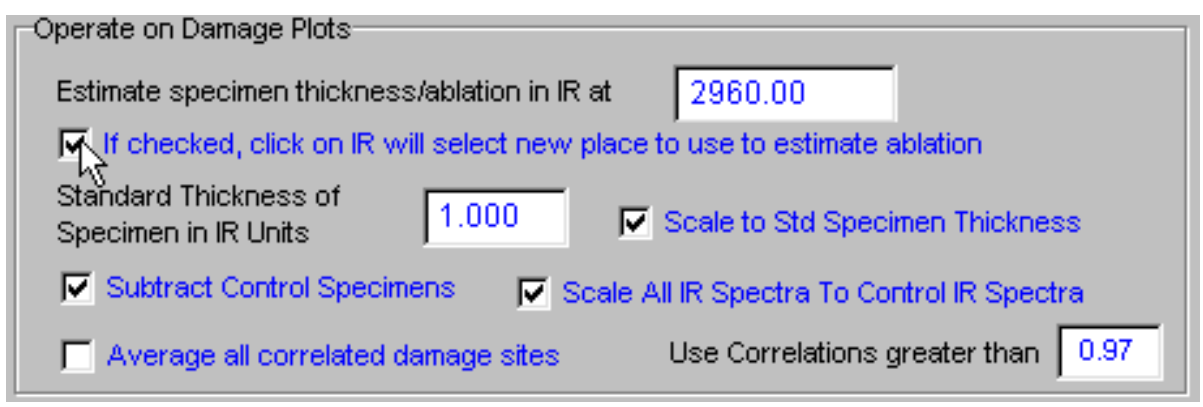



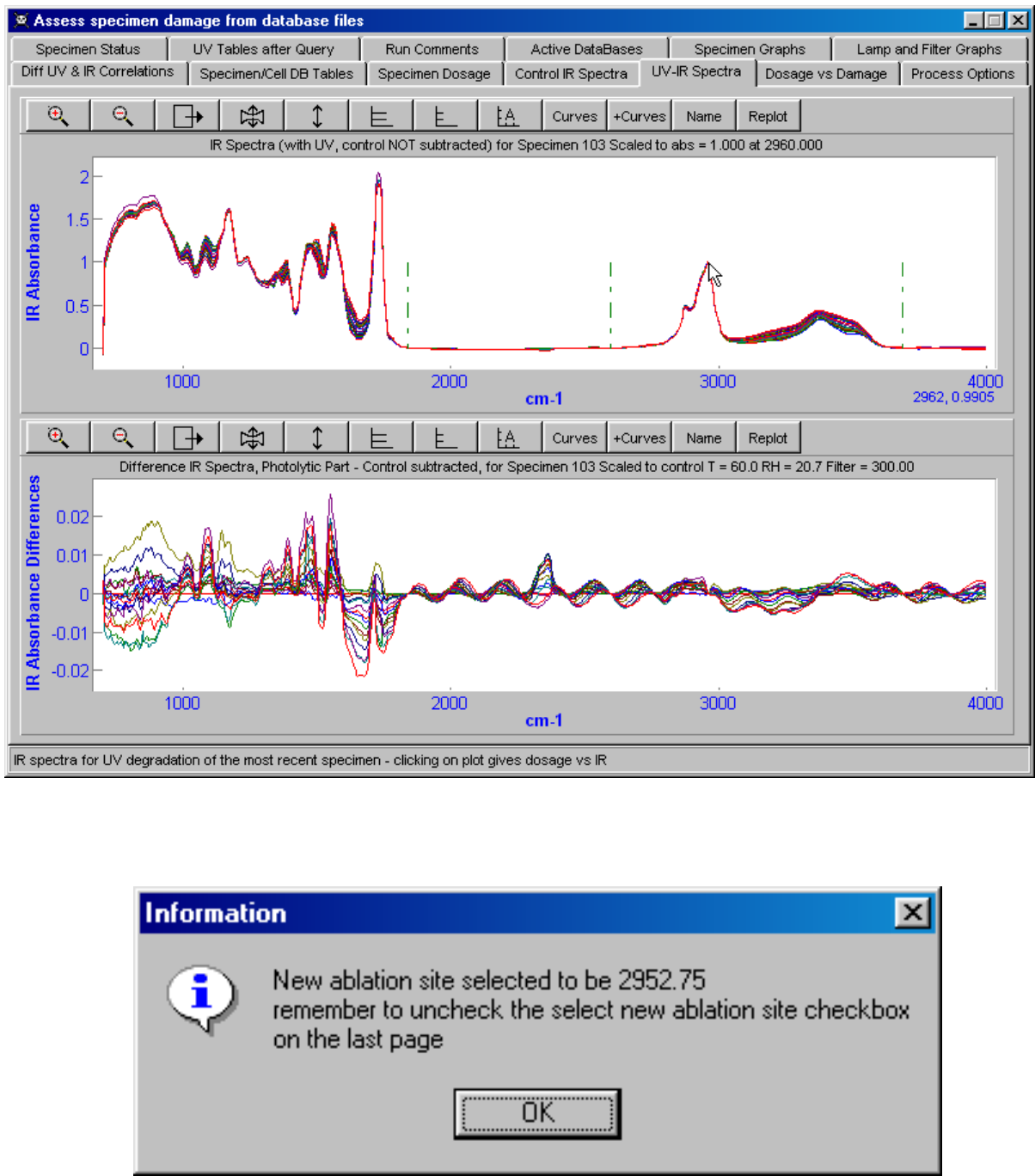

\section{SPECIMEN THICKNESS}

To estimate the damage a specimen has sustained, IR spectra from two specimens must be compared and subtracted. Typically, the control specimen has been kept in the dark and the "damaged" specimen has been exposed to lamp radiation. These specimens were probably not of exactly the same thickness at the beginning of the exposures and the thickness of the exposed specimens may have changed as a result of ablation during the exposure. Thus, the spectra will probably not have equal intensity where they should have equal intensity. If the "Scale to Std Specimen Thickness" check box (see above figure) is checked (by clicking on it), the spectra are scaled (on the vertical axis) to a standard value, specified in the "Standard Thickness of Specimen in IR units" box, at the wavelength specified in the "Estimate specimen thickness/ablation in IR at" box (see the figure above). 
Subtractions of the spectra from one another will then be more realistic. If on the other hand the ablation is of major interest, one would not want to correct for ablation in this way because the effect of ablation would then be removed from the data. The check box allows the program to handle both cases.

\section{Subtracting THE CONTROL SPECIMENS}

The effects in the control specimens are usually subtracted from the exposed specimens - that is why there are controls. None the less, the option is provided not to subtract the controls by unchecking the "Subtract Control Specimens" check box (see the figure above).

\section{Scale ALl IR Spectra to Control Spectra}

If the "Scale to Std Specimen Thickness" check box is not checked, no scaling to the ablation site will be carried out and ablation will be included in the results. Including ablation may override any chemical changes; not including ablation will make the chemical changes the only changes.

\section{SMOOTHING THE SPECTRAL CURVES}

If the "Smooth All Curves" check box is checked, the raw data spectra will be smoothed immediately after they are added to the plots (the data on the disk will remain untouched). Subsequent arithmetic operations using these spectra will then use the smoothed values.

\section{Ablation, Dosage and Damage}

The dosage of a specimen is calculated from the lamp and filter spectra and from the UV spectra of the specimen. Because the beam traversing the specimen is attenuated by the specimen, degradation is probably not uniform across the specimen. Scaling the dosage as a function of specimen thickness is not a simple matter. Therefore, if ablation has taken place and but corrections for ablation are not to be made, the damage spectra are first scaled so that they can be compared with and corrected by reference to the control damage spectra, then, when making the damage/dosage plot, are rescaled back to the values they should have for a specimen of the thickness that produced the dosage.

\section{SignAL VERSUS NOISE}

The BFRL photoreactor and this system of programs are used to assess the weathering resistance of specimens which are presumably very resistant to the effects of weather. Therefore it may be that some aspects of the test do not produce a non-zero result - in other words, the signal is not above the noise. On the other hand, if the signal is not low, the film is probably not worth testing because it is being changed significantly by the exposure and is not very resistant to weathering. Consequently, noise is a problem which must be watched carefully, the experimental conditions must be well-controlled, and the exposures must be long enough for the signal to be significantly above the noise. 
The first test in the fight against propagated noise obscuring the results is in specifying the minimum amount of UV absorbance the specimen is required to have in the region of the filter transmission. This is the quantity specified in the box labeled "Minimum Significant UV Absorbance in Filter Range".

The point is that the specimen UV absorbance must be very well-known for the calculations to be meaningful because the specimen UV absorbance enters the dosage calculations as the exponent of 10 , i.e., as $\mathrm{A}$ in $10^{-\mathrm{A}}$.

\begin{tabular}{|c|c|c|c|c|c|}
\hline \multicolumn{6}{|l|}{ Limits Defining Non-Zero Data } \\
\hline Absorbance in Fitter Range & 0.002 & Absorbance Range & 0.020 & $(+i-)$ will mean a curve is just noise & 0.33 \\
\hline
\end{tabular}

The second test is that the IR absorbance where the damage is to be monitored must change during the experiments by at least the number of absorbance units specified in the box labeled "Minimum IR Absorbance Range"

The third test is on the fraction of times a damage or dosage curve changes direction when plotted against time of exposure. If the two lines joining three adjacent points in the curve have slopes of different sign, this is counted as a sign of noise. The test then examines all sets of three adjacent points. The fraction of the cases where noise is signaled is compared with the number given in the box labeled "More than this fraction of reversals will mean a curve is just noise". A curve which first grew and then declined would always have one such point, where it changed from growing to declining, but many more than one would signify that the curve changed directions many times and presumably was very noisy. If a curve is found to be noisy and automatic processing is underway, the data in that curve will not be processed further. If manual operation is underway, warning messages will be shown.

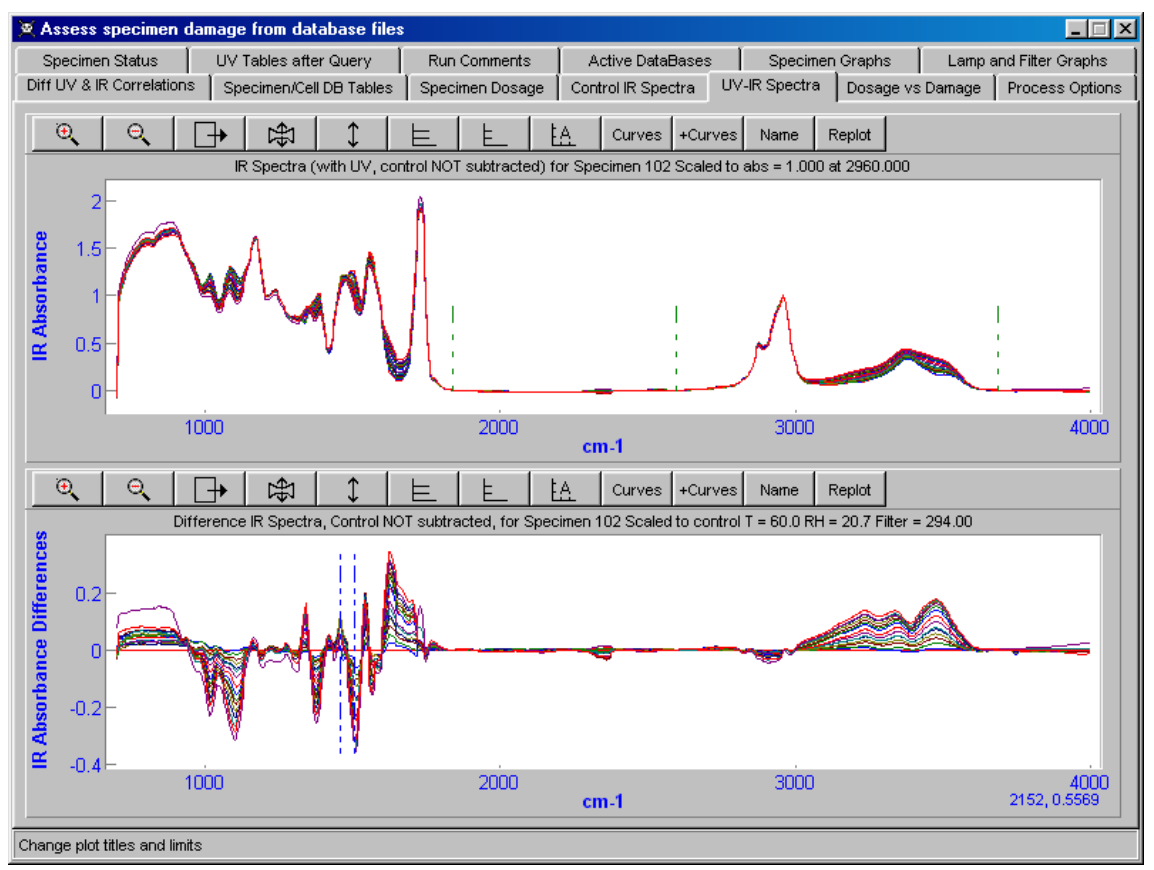


For example, a specimen was processed manually, the dosage was integrated, and the difference damage plot was right-clicked in two places, shown by vertical lines in the next figure.

The right hand line in the bottom plot of the previous figure is on a site of appreciable change in absorbance in the IR spectrum and the trend in absorbance contains only a little noise, shown by the few changes in sign of the slope in the top plot in the next figure.

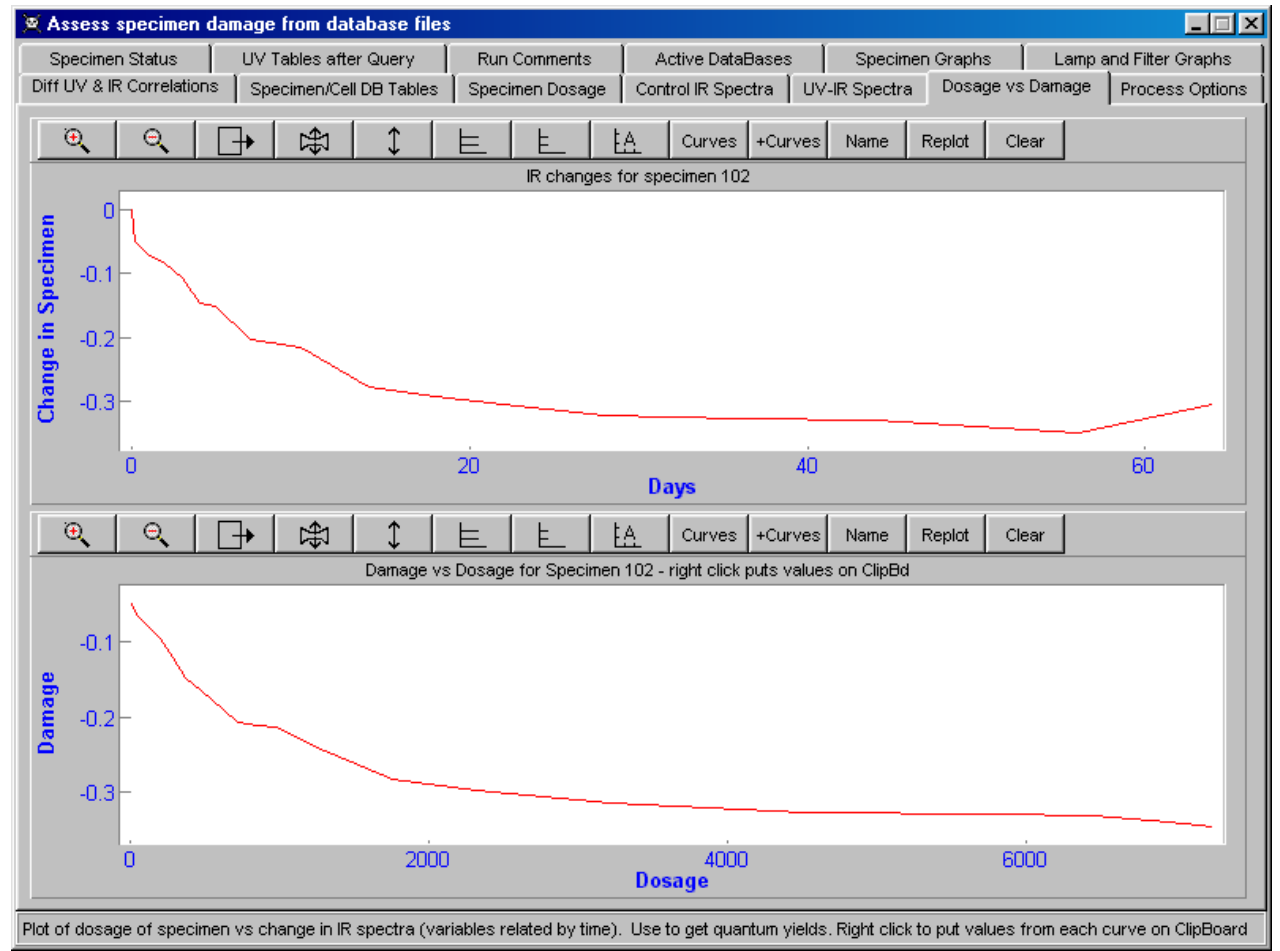

The result of clicking in the figure where the line on the left is (in the second plot in the previous figure) is the dialog box warning

because the damage/time plot, the top plot in the next figure, is too noisy, i.e., the line contains too many reversals in sign of the slope. 


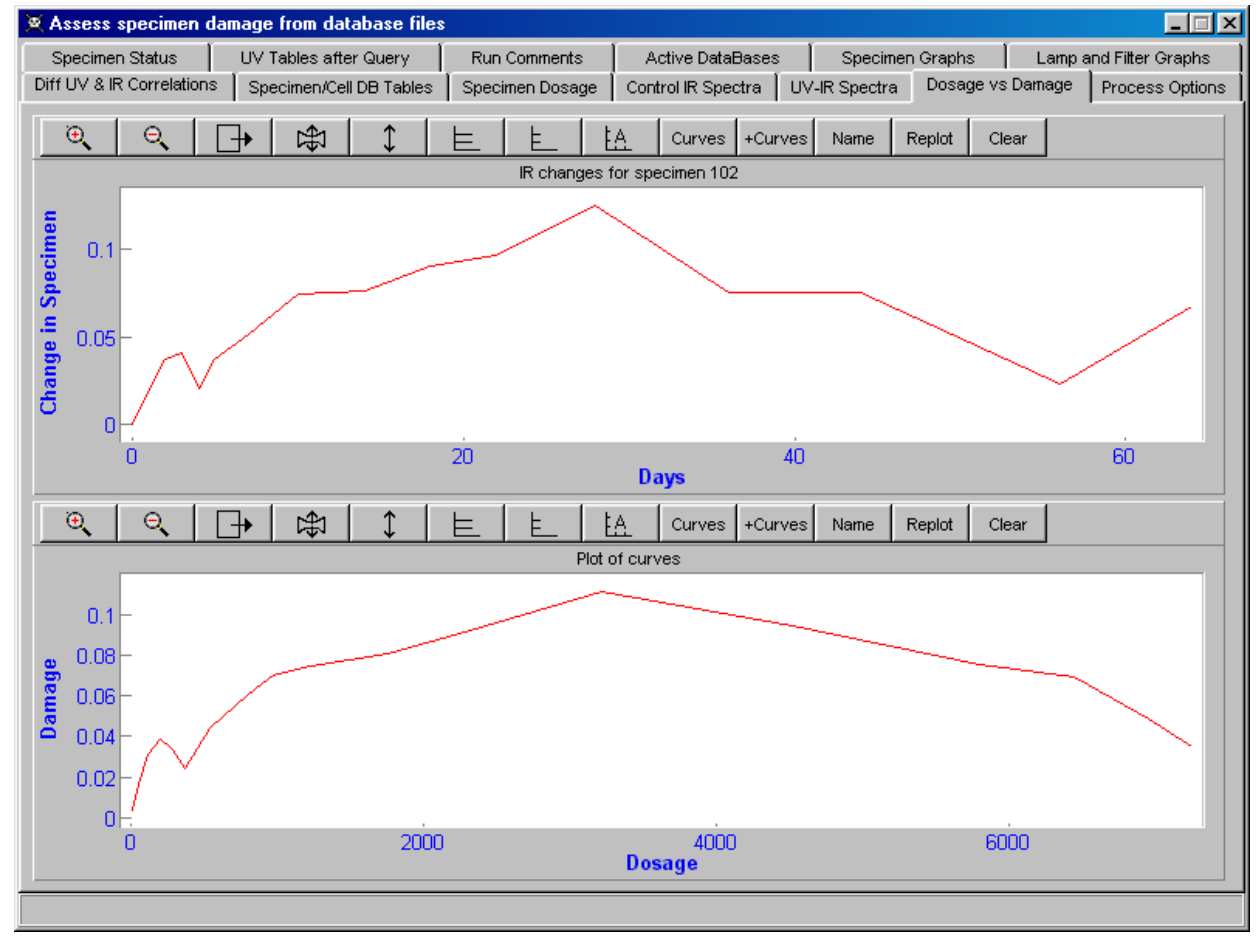

The slope noise criterion on the Process Options page was:

More than this fraction of sign reversals $(+i-)$ will mean a curve 0.33 is just noise

\section{Correlations in the Damage Spectra}

The damage spectra show the changes which occur as the specimens are exposed to a laboratory environment. It may be that the related chemical processes are not immediately obvious. Therefore an option was provided to assess which in which regions in the damage spectra changes are related to each other.

Chemical groups which appear in the specimen during degradation will have increased IR absorbance. Chemical groups which disappear during the degradation will have decreased IR absorbance. It would simpifly the interpretation of the IR spectra if all changes arising from a given process (i.e., the destruction of a chromophore) could easily be identified and treated together. Secondly, it is futile to examine two regions in the damage spectra which are well correlated because one is then using the same information twice. Thirdly, changes in spectra are best measured at absorbances of 0.4 to 0.7 or perhaps as high as 1.0 , 
so the intra-spectra correlations can be used to select a region with the most-nearly satisfactory absorbance.

A system was therefore devised and implemented to assess correlations among absorbances in the damage spectra. There are typically 10 to 20 spectra in a series of damage spectra. For every wavelength, there are therefore 10 to 20 absorbance values which, if plotted with spectrum number $(1,2,3,4 \ldots)$ on the $x$ axis and absorbance on the $y$ axis, constitute a curve. If the user selects a location in the damage spectra (by clicking on the plot of the spectra), the correlations of that site with all other sites (at all other wavelengths) in the IR spectra can be calculated and plotted. This shows which regions of the spectra are correlated with the degradation process at the clicked point, simplifies the interpretation of the spectra, and allows the user to follow damage using the best site, as discussed above.

\section{Correlation in IR Spectra}

Г If checked, click on IR plot gives correlations in IR, otherwise click puts IR into damage plot

Г Data in Correlation Plot Must Pass Noise Test

The first check box sets up the damage plot so that right clicks on it will be interpreted as places for which to calculate the correlation to all other parts of the spectrum. The various correlation curves are plotted in a correlation plot. Uncheck the check box after the tests so that mouse clicks can revert to their normal functions.

If the "Data in Correlation Plot Must Pass Noise Test" check box is checked, the program first checks that the trend with spectrum number (i.e., time) is not noisy according to the fraction of sign reversals permitted for the slope of lines joining adjacent points. If the trend fails the test, no correlation plot will be made. The severity of the test for noise can be reduced by increasing the fraction of allowed reversals (i.e., make the number in the box shown below closer to 1.0).

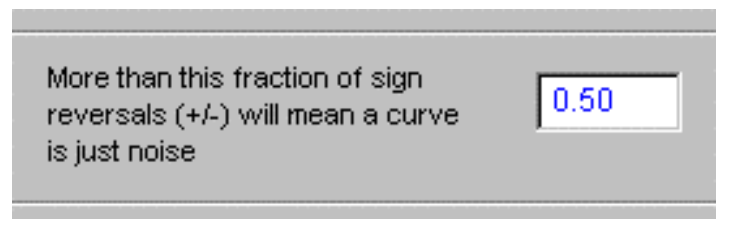

When the correlation plot is being made, trends in the spectra which do not pass this same noise test will have their correlation with the trend at the point clicked set to zero.

As an example of a correlation plot, a right click on the following spectrum at $1674 \mathrm{~cm}^{-1}$, the right-hand peak of the double-peaked envelope near the middle of the plot, 


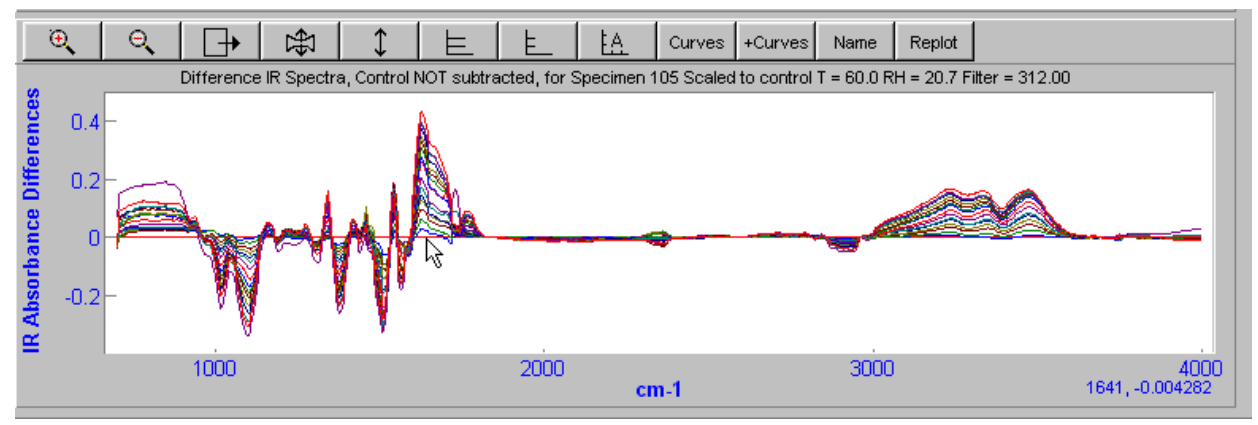

gives the following correlation plot:

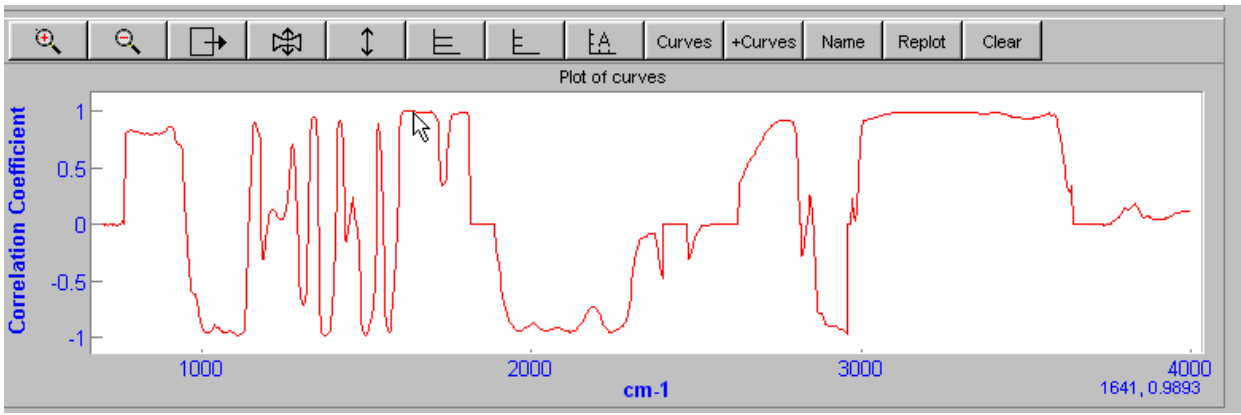

Places in the correlation plot where the vertical coordinate is at or near +1 contain the same trend with time (i.e., with damage spectrum number) as the absorbance at $1674 \mathrm{~cm}^{-1}$, although the actual values of the IR absorbances are almost certainly different. Places where the vertical coordinate is at or near -1 have the opposite trend with time as the series of IR absorbances at $1674 \mathrm{~cm}^{-1}$ and may be from a group which is consumed when the group absorbing at $1674 \mathrm{~cm}^{-1}$ is produced.

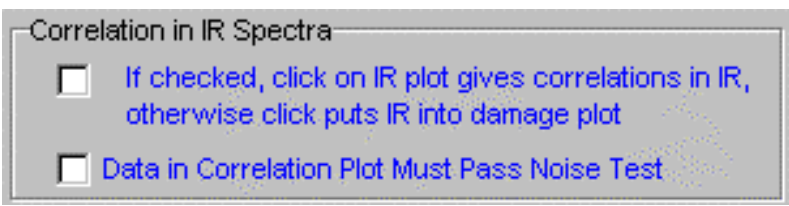

Make sure the "If checked, click on IR plot gives correlations..." check box is unchecked before proceeding.

\section{Contents of the INI file}

A typical INI file should contain entries like those in the following list. If either the file ends before all the needed information has been read or for some reason the wrong kind of information was found (for example a number instead of a table name), the program will not process data until the missing information has been supplied. The information can easily be changed using the interface options described earlier in this guide. The text inside the \{\} brackets is not present in an actual INI file but is used here to document the lines in the file. 
The INI file contains the setting of the various check boxes, the contents of the text edit boxes which present choices to the user, and the names of the files containing the database tables. A different INI file may be

saved for each set of database tables using the

\section{Inv file Menu... -}

09/26/1996 \{beginning date to use in selecting spectra\}

11:00AM $\quad$ \{beginning time to use in selecting spectra\}

09/27/1996 \{ending date to use in selecting spectra\}

12:00PM $\quad$ ending time to use in selecting spectra\}

$Y \quad\{Y=$ Ignore beginning and ending times, do all of specimen spectra\}

$\{\mathrm{N}=$ Do not ignore beginning and ending times $\}$

$342 \quad$ \{low end of dosage integration range in $\mathrm{nm}$ \}

$558 \quad$ \{high end of dosage integration range in $\mathrm{nm}$ \}

0.002 \{minimum UV absorbance range for signal>noise\}

$0.020 \quad$ minimum IR (damage) absorbance range for signal>noise

$0.33 \quad$ \{fraction of sign reversals allowed for signal>noise

$200.00 \quad$ \{where to zero the lamp spectra\}

$450.00 \quad$ \{where to zero the specimen spectra

$2960.00 \quad$ site in IR spectrum to use to estimate or correction for ablation\}

$1.000 \quad$ \{scale to this standard IR absorbance for thickness\}

$0.100 \quad$ \{low fraction of dosage at which to get quantum yield at\}

$0.150 \quad$ \{high fraction of dosage at which to get quantum yield at\}

$0.050 \quad$ \{low fraction of dosage range in fitting polynomial\}

$0.900 \quad$ \{high fraction of dosage range in fitting polynomial\}

$\mathrm{N}$

\{is New Ablation Site Check Box checked? (Yes or No)\}

Y

\{is Scale To Std Thickness Check Box checked? (Yes or No)\}

Y

\{is Subtract Non-UV Samples Box checked? (Yes or No)\}

Y

\{is Scale To Non-UV Check Box checked? (Yes or No)\}

1839.65 \{first of spectral sites to zero damage spectra at\}

2594.53 \{second of spectral sites to zero damage spectra at\}

$3686.50 \quad$ \{third of spectral sites to zero damage spectra at\}

allowed\}

\{blank line to signify end of zero sites information - 50 sites

$1084.78 \quad$ \{first of spectral sites to assess damage at\}

1629 \{second of spectral sites to assess damage at\}

1674.16 $\quad$ third of spectral sites to assess damage at\} 
50 sites allowed

\{blank line to signify end of spectral damage sites information -

\{the following lines are the file names of the various database tables. In the case shown here, the names are self-descriptive.\}

$\mathrm{H}$ :IBFRL Exposures - HalcyonITest \#3IDatabase FilesICellPositions.DBF

H:I BFRL Exposures - HalcyonITest \#3IDatabase FilesIFilterSpectra.dbf

H:I BFRL Exposures - HalcyonITest \#3IDatabase FilesISpecimenID.dbf

H:I BFRL Exposures - HalcyonITest \#3IDatabase FilesISpecimensCells.dbf

H:\ BFRL Exposures - HalcyonITest \#3IDatabase FilesILampsOnOff.dbf

H:\BFRL Exposures - HalcyonITest \#3IDatabase Files|Lamplntensity.dbf

H:I BFRL Exposures - HalcyonITest \#3IDatabase FilesISampleSpectra.dbf

$\mathrm{H}: \backslash$ BFRL Exposures - HalcyonITest \#3\Database Files|Pos2Lamps.dbf

H:\ BFRL Exposures - HalcyonITest \#3IDatabase FilesILAMPPOWER.DBF

$\mathrm{H}: \backslash \mathrm{BFRL}$ Exposures - HalcyonlTest \#3\Database FileslIRSpectra.dbf

$\mathrm{H}: \backslash$ BFRL Exposures - HalcyonITest \#3IDatabase FilesIFilter ID.dbf

H:\BFRL Exposures - Halcyonldamage.dbf

\{end of the INI file\} 


\section{Choosing the Specimen and Time Options}

These options are found on the specimen status page.

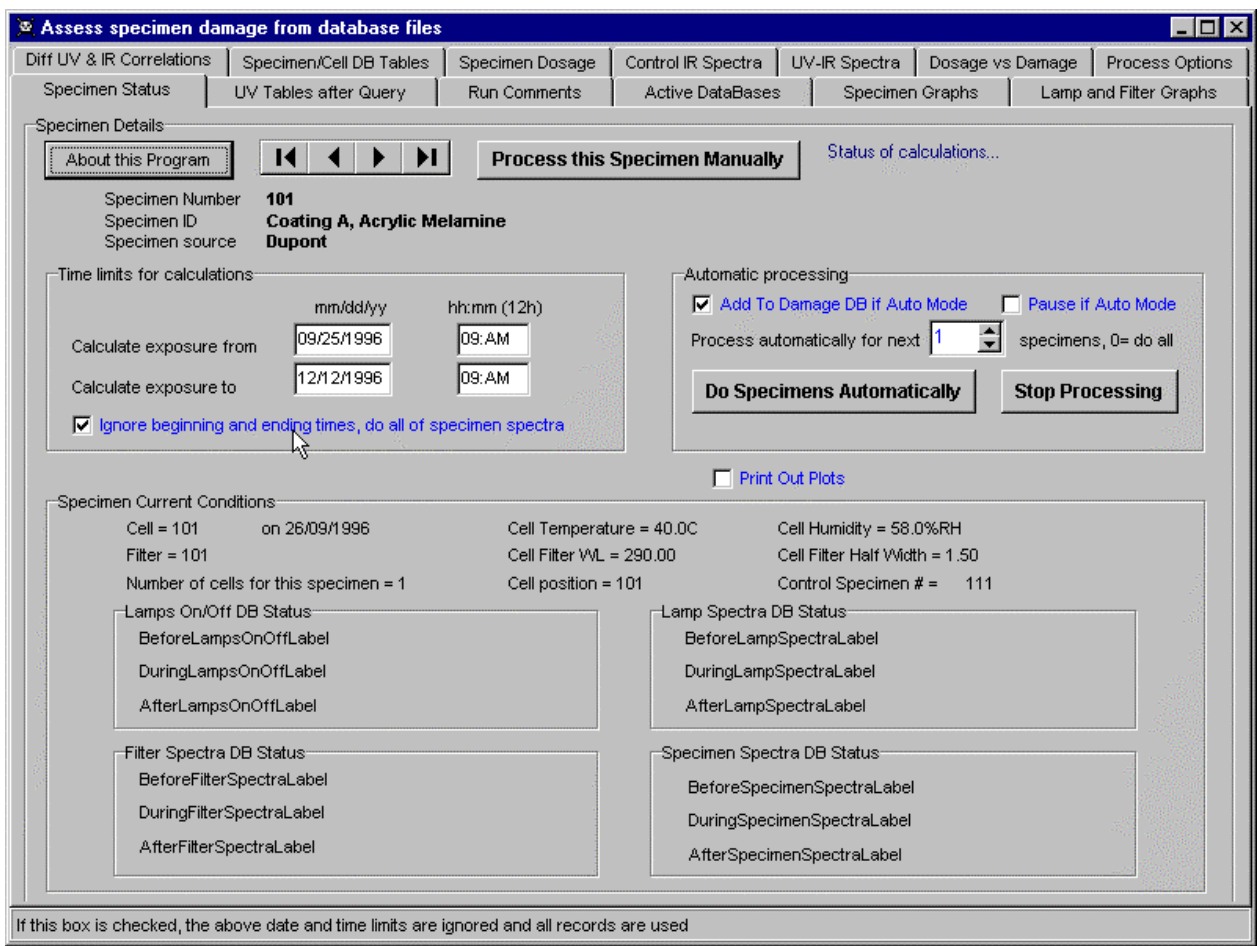

When all the required database tables have been read in, the specimen to process may be selected by clicking on the navigator bar.

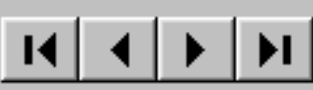

The specimen number, ID and source are updated with each click on the navigator bar.

\begin{tabular}{|c|c|c|c|c|c|}
\hline About this Program & 14 & 4 & $\triangleright$ & D & Pro \\
\hline Specimen Number & \multicolumn{5}{|l|}{101} \\
\hline Specimen ID & \multicolumn{5}{|c|}{ Coating A, Acrylic Melamine } \\
\hline Specimen source & \multicolumn{5}{|c|}{ Dupont } \\
\hline
\end{tabular}

The exposure conditions of the cell in which the specimen was exposed are also updated.

\begin{tabular}{|c|c|c|c|}
\hline \multicolumn{4}{|l|}{ Specimen Current Conditions } \\
\hline Cell $=101 \quad$ on $9 / 26 / 96$ & Cell Temperature $=40.0 \mathrm{C}$ & Cell Humidity $=58.0 \% \mathrm{RH}$ & \\
\hline Filters : 101 & Cell Fitter $\mathrm{WL}=290.00$ & Cell Filter Half Width $=1.50$ & \\
\hline Number of cells for this specimen $=1$ & Cell position = 101 & IR with no UV Specimen \# = & 111 \\
\hline
\end{tabular}




\section{Choosing the Processing Options}

The processing options are on the last page of the program - "Process Options"

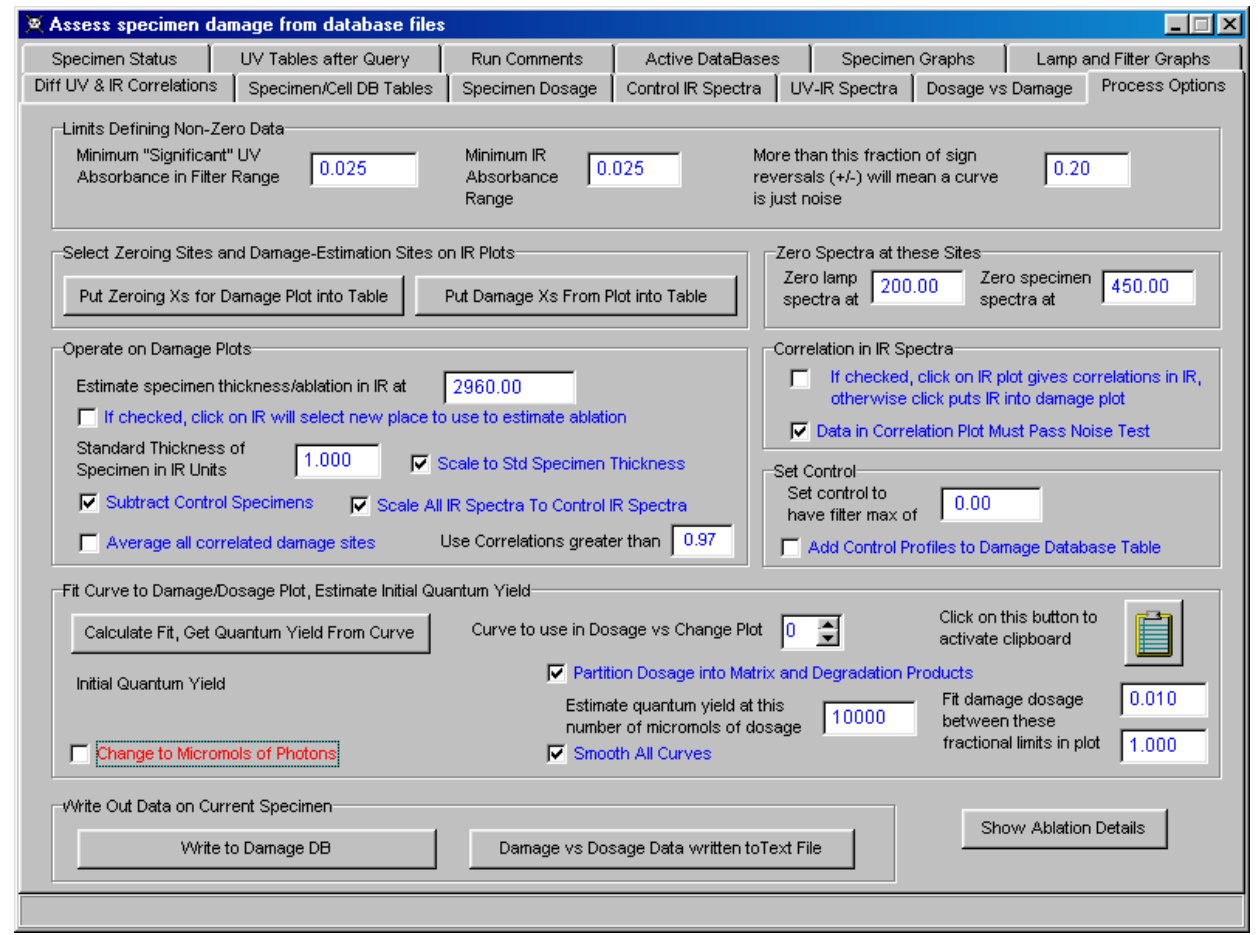

The options which govern scaling of spectra and subtraction of controls from specimens have been covered in the sections on Ablation, etc.

The red "Change to micomols of photons" checkbox allows the program to try to convert the dosage from Joules into photons using the center wavelength of the filter as a means of estimating the energy per photon. In the following examples, the dosage was left in Joules.

\section{Automatic Processing}

For automatic processing, the user must go to the "Specimen Details" page and specify

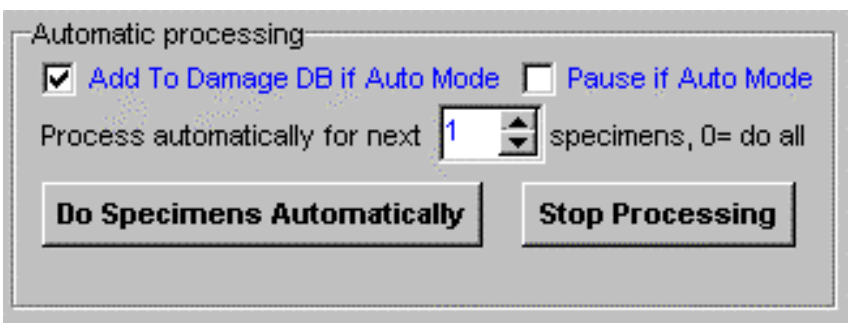


1) how many specimens from the current specimen to process (selecting zero processes all specimens from the current specimen to the end of the table)

2) whether to add the results to a damage database table

3) whether to let the program calculate as quickly as possible or to make it pause for a few seconds at each stage of the calculations so progress can be monitored visually

4) the time period to be covered. Although beginning and ending times and dates can be specified, it is almost always more convenient to check the check box to make the program use all the relevant spectra in the database. (12h) means give the times in AM/PM format.

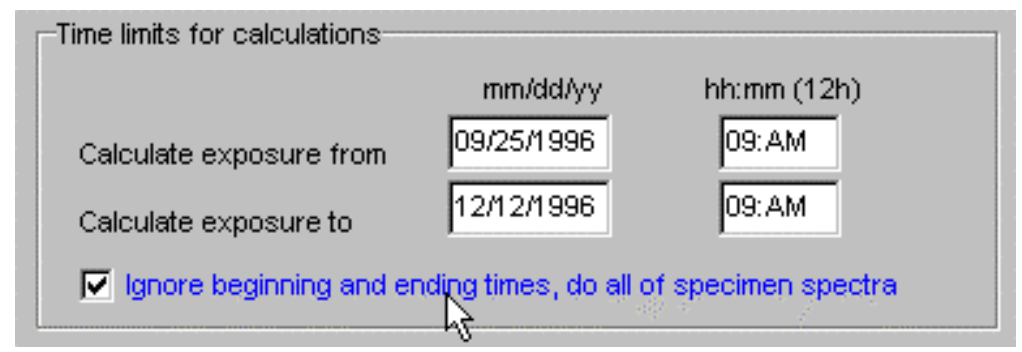

Clicking on "Do Specimens Automatically" starts the process. The process may be interrupted by clicking on the "Stop Processing" button.

\section{Do Specimens Automatically Stop Processing}

\section{Manual Processing}

The various steps involved in manual processing will now be described in some detail to explain the processes involved. The first step is to go to the Specimen Status page and use the navigator bar to choose the specimen to process. Then click on the "Process this Specimen Manually" button.

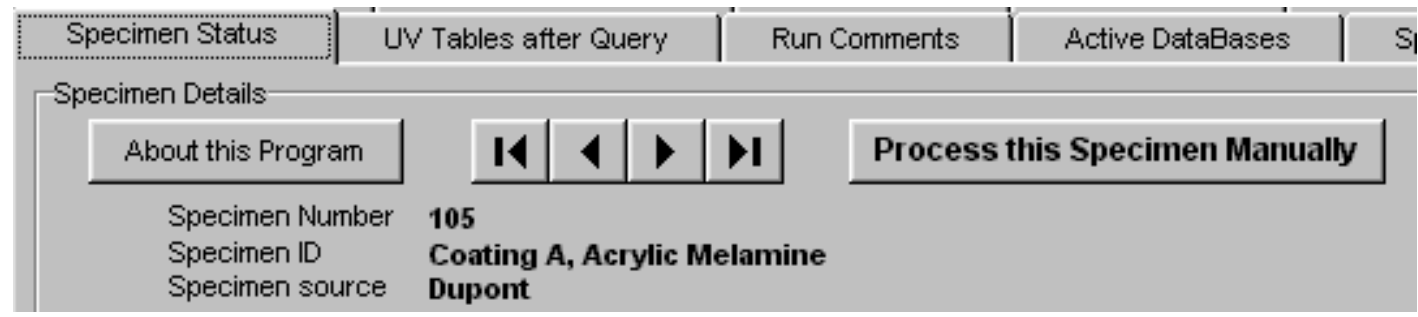

The program searches the database tables for records containing Lamp On/Off times and Lamp, Filter and Specimen spectra. The numbers found are shown in the lower half of the page. The spectra corresponding to each Lamp Off period (when the spectra were 
measured) are found, read in, plotted, and processed as necessary. The status of the calculations is shown in the upper right hand corner of the page.

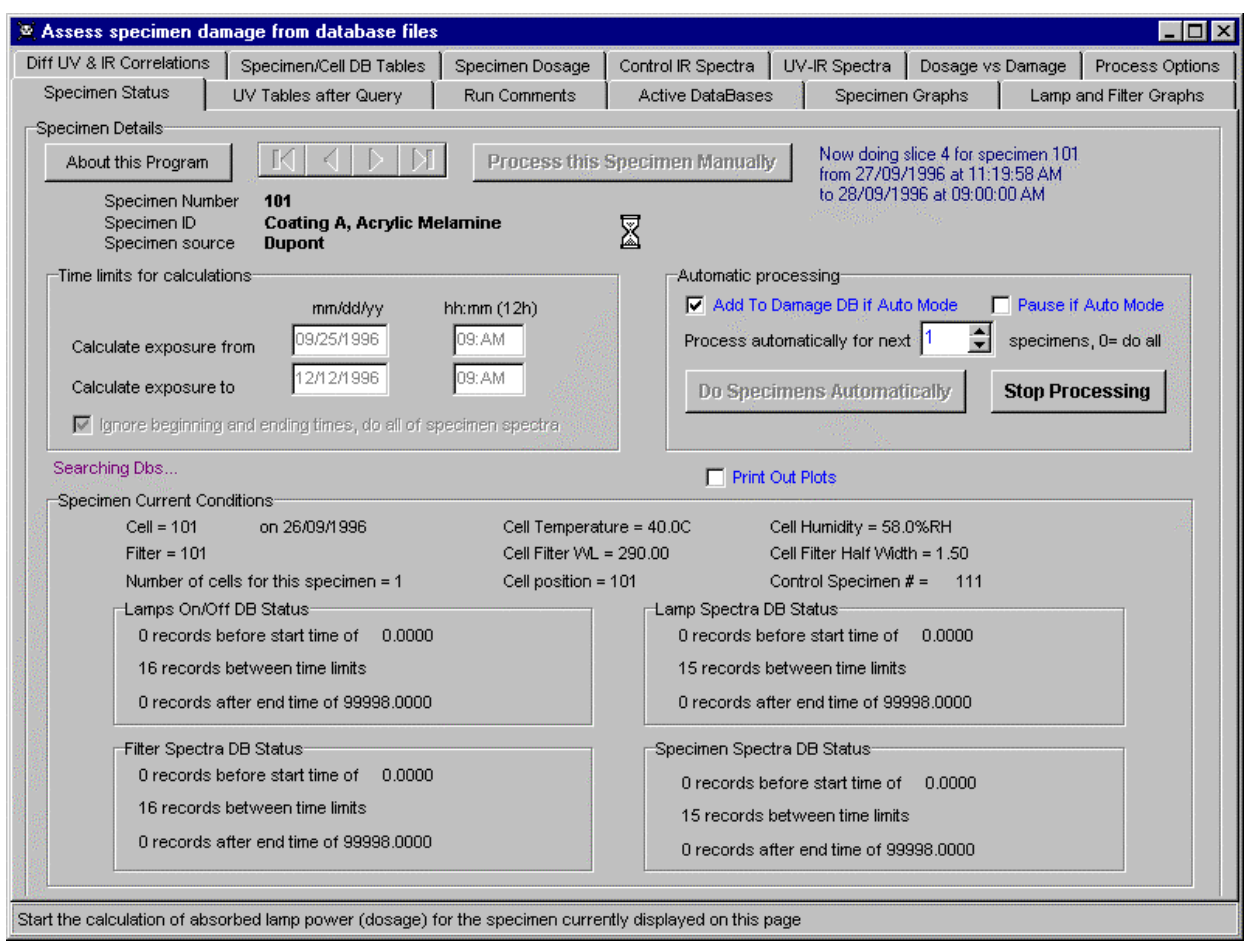

\section{Details of the Calculations}

Radiation from the lamp is directed to the cell containing the specimen, where it passes through a filter and is incident on the specimen. The specimen absorption spectrum gives the fraction of the incident radiation that emerges from the other side of the specimen.

The section "Estimation of dosage, damage, and quantum yield " describes how the spectra from the lamp, filter and specimen are combined to give the dosage, i.e., the amount of radiation which remains in the specimen.

The lamp is one of the components of the experiment which change with time. Below is a plot of lamp spectra taken over several weeks. One of the assumptions in the experimental setup is that changes with time will be monitored frequently enough that any change between monitorings can be assumed to be linear. To monitor the lamp, filter and specimen, the exposure must be stopped temporarily and UV and IR spectra must be taken. The stoppages are recorded in the lamp on/off database table as lamp on (true or false) and time/date. The times and dates in all these database tables are stored in days and fractions of days since 1900 and are precise to about 10 seconds. This method can record elapsed time for approximately 2600 more years (999999/365 100 = space in DB/days per year - those years already gone) and is expected to be sufficient for the life of the program. 


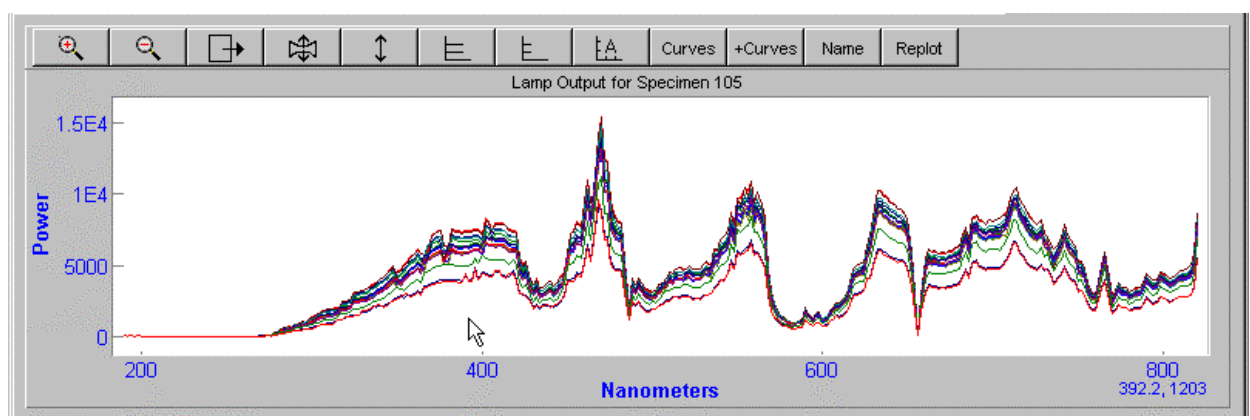

The program first checks whether it is to use only those spectra in a given time span (given on the "Specimen Status" page) or all spectra. Then it looks for the appropriate spectra in the appropriate database table. While the program has to take the information in the tables as "gospel", it performs several checks on the data. For example, the dates/times of the spectra are adjusted to be the times of the lamp on/off periods, as shown below.

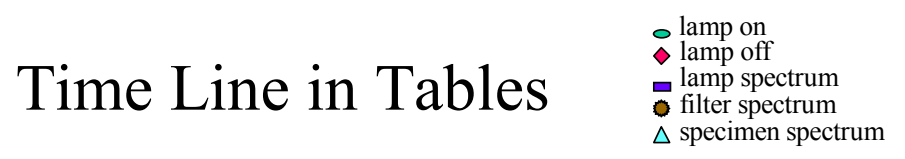

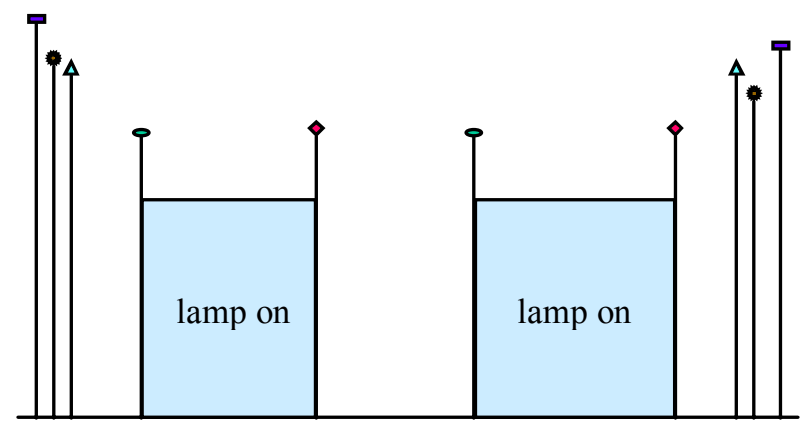

time

In addition to recording the state of the components of the apparatus by taking their spectra, damage in the specimens has to be monitored. For these spectra to be taken, the exposure has to be stopped from time to time. The above figure shows as an example that the exposure was stopped twice but for some reason spectra are only available before and after the two exposure time slices. (This is for purposes of demonstration only. It is recommended that spectra be taken every time the exposures are stopped. All adjustments are made to the data in memory. The original database files remain untouched.) 

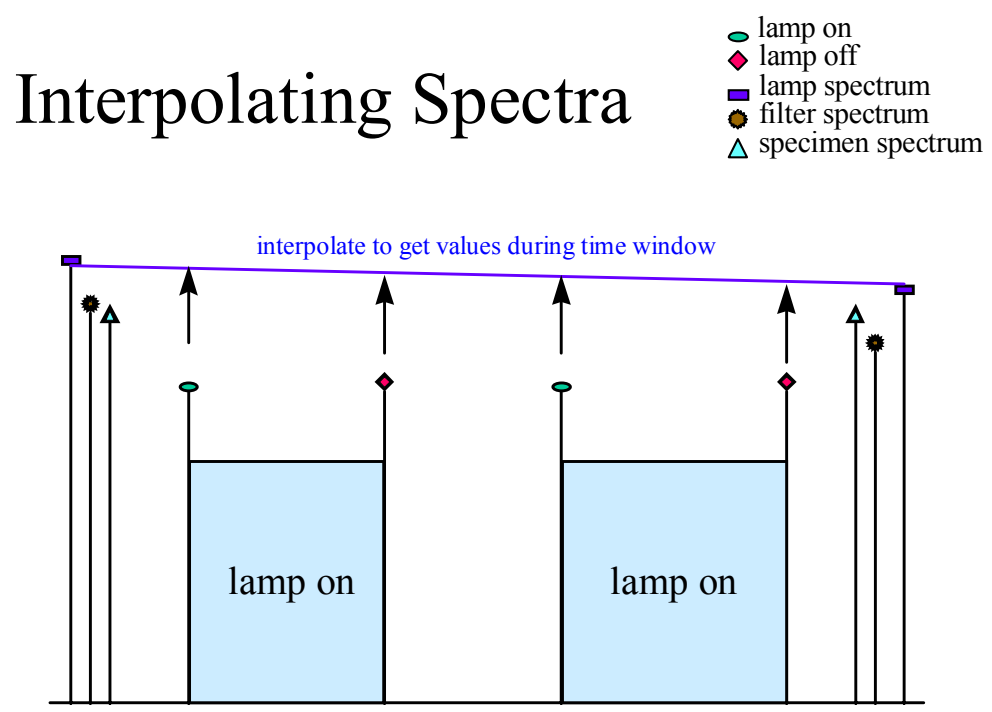

time

First, there are no spectra between the two sets of lamp-on times. Therefore, the spectra will be interpolated across the time interval to assess the values they would have had if they had been taken at the time in the middle of the figure.
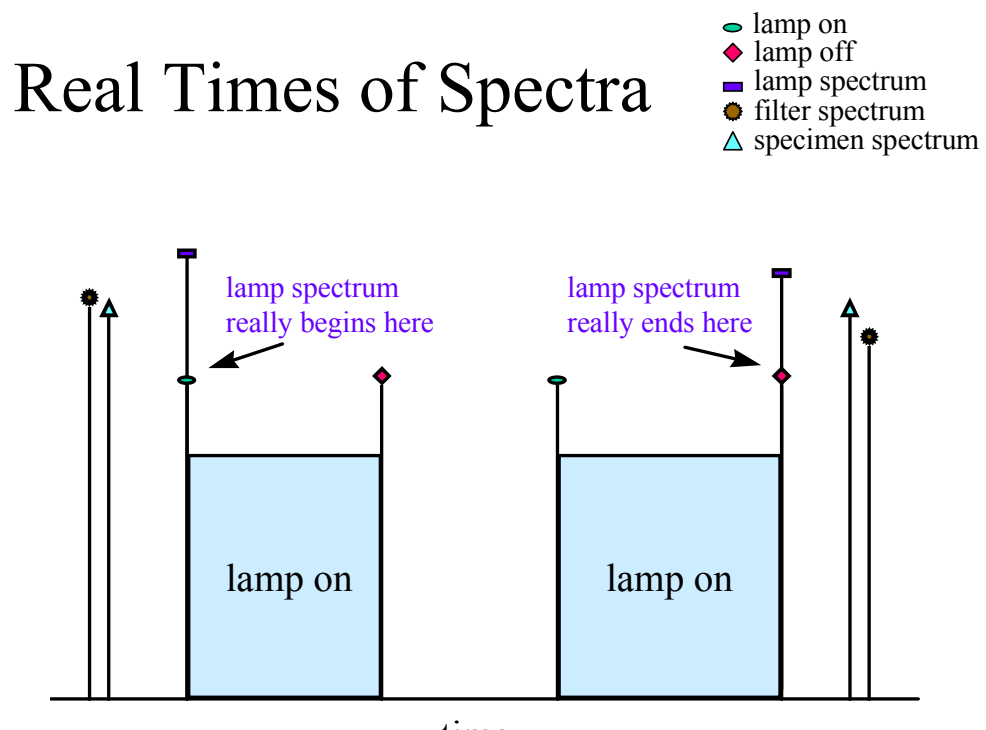

time

However, it is not quite that simple. There was no photolytic exposure of the specimen while the lamp was off so the time to use for the lamp spectrum is when the lamp was actually switched on and off, not the times the spectra were measured. 

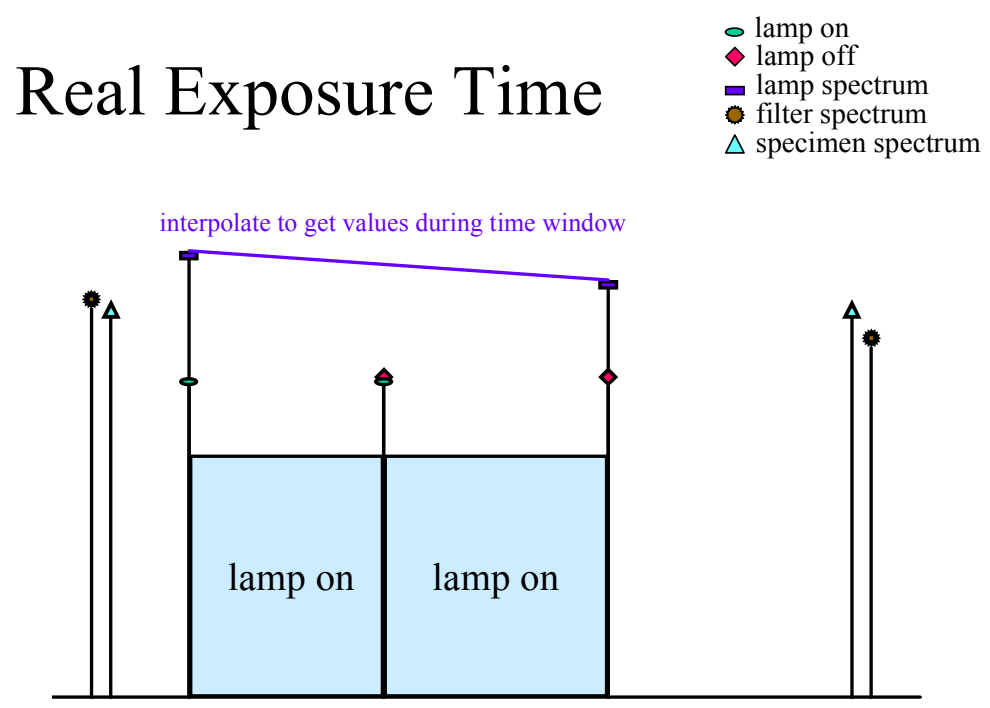

time

Now the dead time between exposures has to be eliminated.
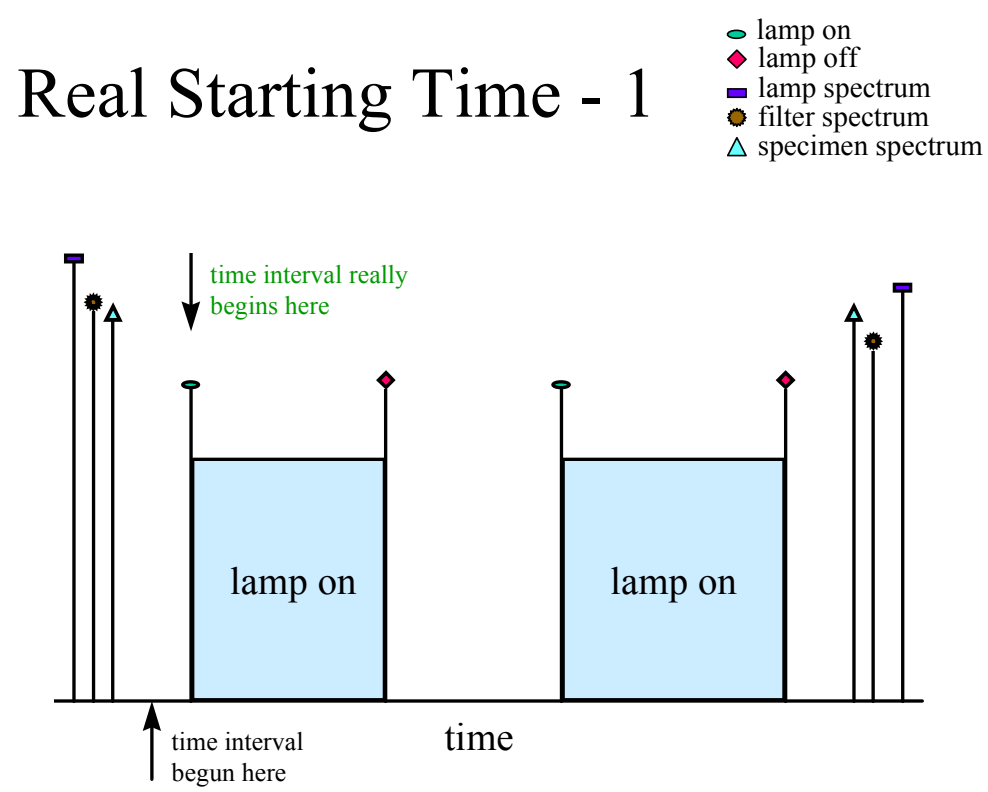

As shown below, the starting time of the exposure can be specified at any time before the exposures actually began, but it has to be adjusted to the time the lamp was first switched on. 


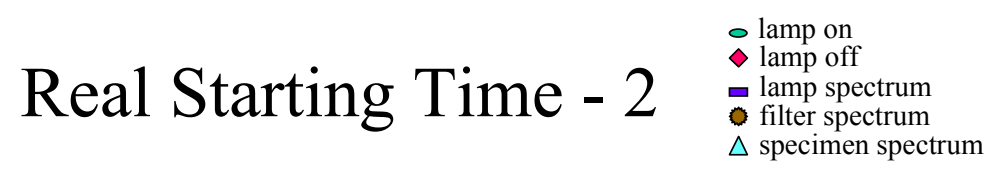

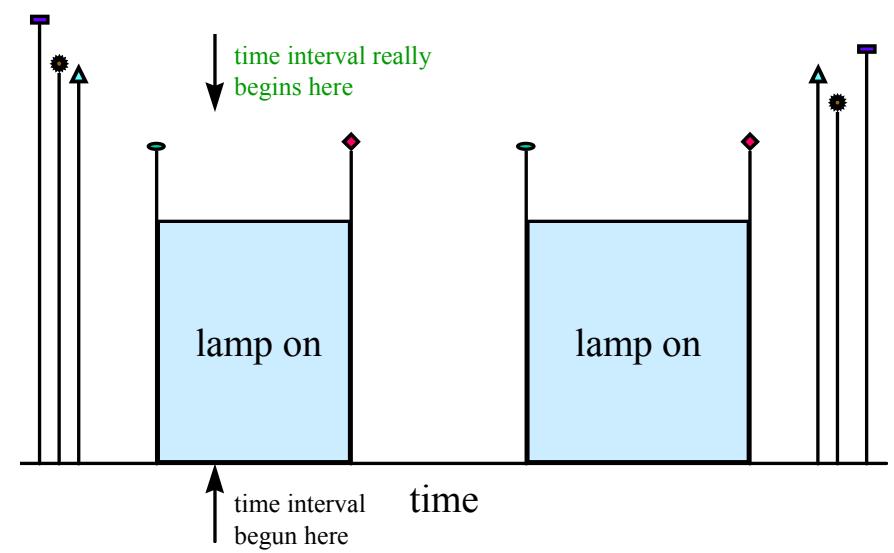

Depending on where the user wants to begin the calculations, there are other possibilities to consider. If the time the user specified was "inside" an exposure interval, that time must be maintained.

In practice, the program finds two UV-visible spectra for the lamp, the filter, and the specimen for each time slice when the lamp was on. One spectrum reflects the state of the lamp, filter or specimen before the lamp was switched on and the second spectrum reflects the state of the item after the lamp was switched off. If a spectrum is missing, the program uses spectra from earlier or later, as need be, taking into account the increased time span. The progress of its search and the results of its decisions are documented in the "Run Comments" page. 
Assess specimen damage from database files

\begin{tabular}{|l|l|l|l|l|l|l|}
\hline Diff UV \& IR Correlations & SpecimeniCell DB Tables & Specimen Dosage & Control IR Spectra & UV-IR Spectra & Dosage vs Damage & Process Options \\
\hline
\end{tabular}

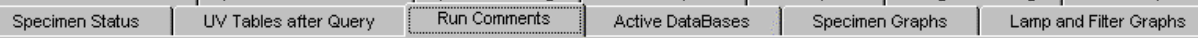

\section{\begin{tabular}{|l|l|l|l|l|}
\hline Print Run Comments & Export Dosage Graphs As ASCII & Print Graphs \\
\cline { 3 - 4 }
\end{tabular}}

UV Exposures calculation comments for run on 07/09/1998 08:03:21 PM

Specimen number 101

Lamp number

Filter number 101
for exposures between 30/12M899 12:00:00 AM to 12M0/2173 12:00:00 AM

Numerical times are from 0.0000 to 99998.0000

Lamp number for cell position 101 is 1

Lamp on time for lamp number $1=35333.3750$

Lamp was initially off

Lamp number for cell position 101 is 1

316 Lamp Intensity To Power Conversion Factors read from DE H:ICBT Exposures - HalcyoniTest \#2Database FilesLampPower. dbf -

Now doing slice 1 from 25/09/1996 at 09:00:00 AM to 25/09/1996 at 01:00:02 PM

Numerical times are from 35333.3750 to 35333.5417

Lamp "earlier" spectrum not "aged"

i.e., spectrum was found in preceeding lamp offion time interval,

Lamp "later" spectrum not "aged",

i.e., spectrum was found in following lamp offion time interval,

Filter "earlier" spectrum not "aged"

i.e., spectrum was found in preceeding lamp offion time interval,

Filter "later" spectrum not "aged",

i.e., spectrum was found in following lamp offion time interval,

Specimen "earlier" spectrum not "aged"

i.e., spectrum was found in preceeding lamp offion time interval,

The relevant information in the database tables is shown on the "UV Tables after Query" page and the "Specimen/Cell DB Tables" page. These pages can be consulted if the calculations seem to have gone awry. In that case, the first thing to check is whether something is wrong in the database tables. The database tables contain a large amount of information. It is unlikely that all this information has been gathered and entered into the database tables without some mistakes being made.

Assess specimen damage from database files

\begin{tabular}{|l|l|l|l|l|l|l|}
\hline Diff UV \& IR Correlations & SpecimeniCell DE Tables & Specimen Dosage & Non-UV IR Spectra & UV-IR Spectra & Dosage vs Damage & Process Damage
\end{tabular}

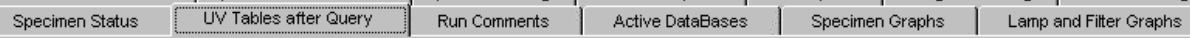

Lamp On/Off DB status

\begin{tabular}{|c|c|c|c|}
\hline LAMP_NUM & ON & WHEN & $\triangle$ \\
\hline 1 & $T$ & 35529.3333 & \\
\hline 1 & $\mathrm{~F}$ & 35529.5000 & \\
\hline 1 & $\mathrm{~T}$ & 35529.6736 & \\
\hline 1 & $F$ & 35530.3333 & \\
\hline 1 & T & 35530.5035 & \\
\hline 1 & $F$ & 35531.3333 & $\nabla$ \\
\hline
\end{tabular}

Position to Lamp Number Correspordence

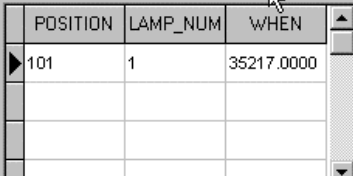

Lamp Power Conversion Factors LAMP_NUM WAVELNGTI WRFACTOH -

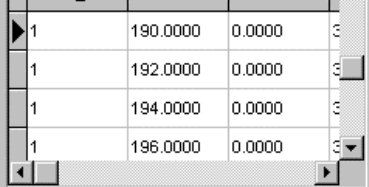
Lamp Spectra DB status

\begin{tabular}{|l|l|l|l|l|l|l|l|}
\hline & TYPE_NUM & WHICH & WHEN & X0 & DELTA_X & NUM_PTS & PECTRUM___ \\
\hline 1 & 101 & 35529.3333 & 190 & 2 & 316 & --MEMO--- \\
\hline 1 & 101 & 35529.5000 & 190 & 2 & 316 & --MEMO--- \\
\hline 1 & 101 & 35530.3333 & 190 & 2 & 316 & --MEMO--- \\
\hline 1 & 101 & 35531.3333 & 190 & 2 & 316 & --MEMO--- \\
\hline 1 & & & & & &
\end{tabular}

\section{Filter Spectra DB status}

\begin{tabular}{|l|l|l|l|l|l|l|l|}
\hline TYPE_NUM & WHICH & WHEN & X0 & DELTA_X & NUM_PTS & PPECTRUM__- \\
\hline 2 & 101 & 35529.3333 & 190 & 2 & 316 & --MEMO--- \\
\hline 2 & 101 & 35529.5000 & 190 & 2 & 316 & --MEMO--- \\
\hline 2 & 101 & 35530.3333 & 190 & 2 & 316 & --MEMO--- \\
\hline 2 & 101 & 35531.3333 & 190 & 2 & 316 & --MEMO--- \\
\hline 1 & & & & & &
\end{tabular}

Specimen Spectra DB status

\begin{tabular}{|l|l|l|l|l|l|l|}
\hline TYPE_NUM & WHICH & WHEN & X0 & DELTA_X & NUM_PTS & PECTRUM__- \\
\hline 3 & 101 & 35529.3333 & 190 & 2 & 316 & --MEMO--- \\
\hline 3 & 101 & 35529.5000 & 190 & 2 & 316 & --MEMO--- \\
\hline 3 & 101 & 35530.3333 & 190 & 2 & 316 & --MEMO--- \\
\hline 3 & 101 & 35531.3333 & 190 & 2 & 316 & --MEMO--- \\
\hline 1 & & & & & &
\end{tabular}




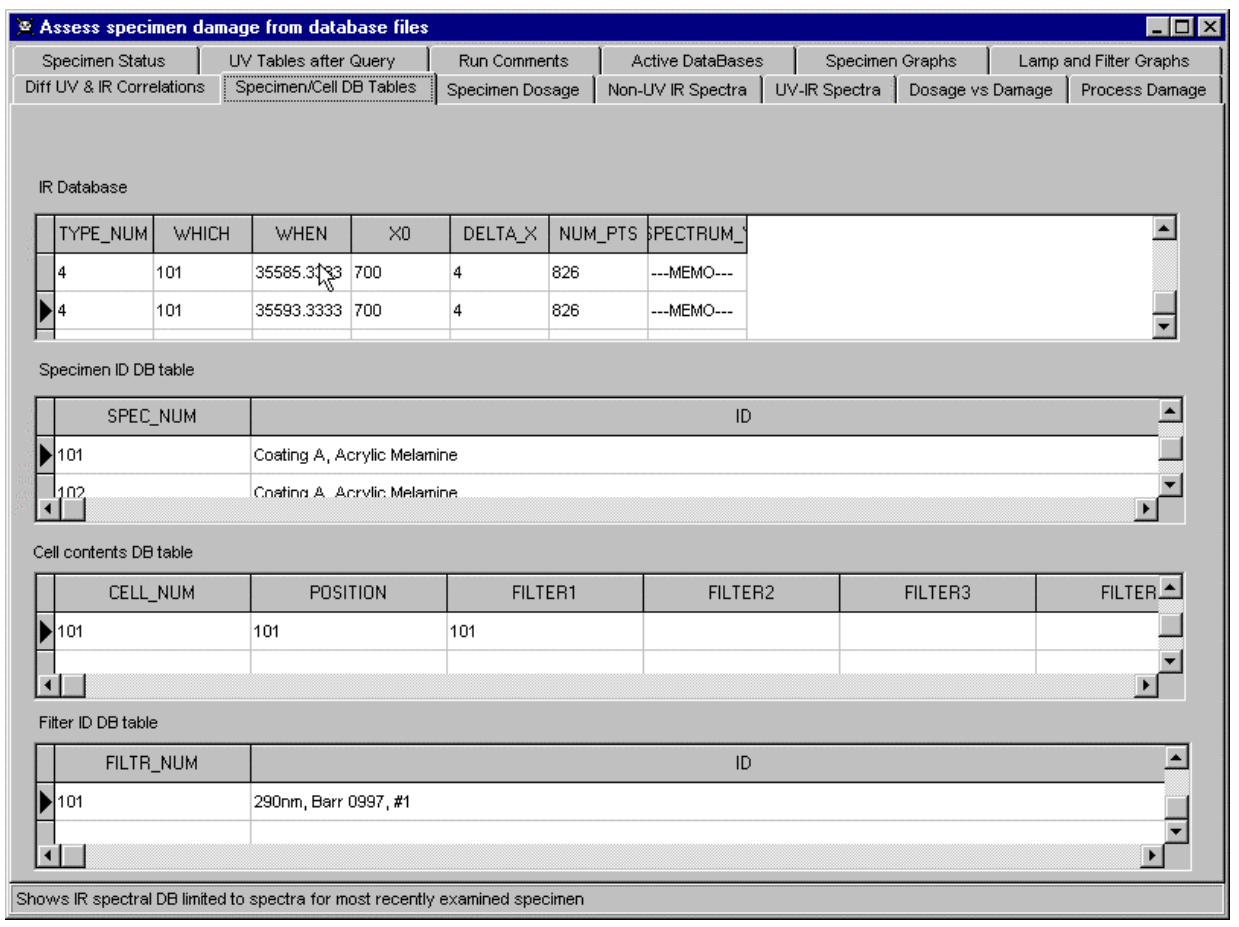

From each pair of lamp spectra the program must estimate the irradiance, $\mathrm{I}_{0}$, in watts incident on the filter which is on top of the cell.

The filter spectra contain the transmittance of the filter. These two spectra must be multiplied together to estimate the radiation flux transmitted through the filter (see the section on Estimation of Dosage, Damage, and Quantum Yield.). However, for each wavenumber point in the spectra, we have a reading from before the period began and a reading from after the period was over. Thus, for each wavenumber in the spectra, we have to multiply two sloping lines, one between the before and after readings for the lamp, and one for the before and after readings for the filter. Experimentation with a spreadsheet was used to generate the table below, which compares the differences obtained for multiplying together two sloping lines (e.g., changing lamp intensity and changing filter transmission) with the beginning and ending values ("initial" and "final" columns in the table below) given in the first four columns of the table. The difference columns were obtained using the average values of the lines, using 3 interpolation sites along the lines and using 5 interpolation sites along the lines, assuming that using 10 interpolation sites along the lines gives a good estimate of the desired product. The reason the averaging is a little different when the averages are used is that interpolation involves calculating $\Sigma a_{i} b_{i}$ whereas the averaging method calculates $\Sigma a_{i} \Sigma b_{j} / n$, which incorrectly includes the cross-terms $a_{i} b_{j}$. 


$\begin{array}{lllllll}\text { initial 1 } & \text { final 1 } & \text { initial 2 } & \text { final 2 } & \begin{array}{l}\text { \%difference } \\ \text { (average) }\end{array} & \begin{array}{l}\text { \%difference } \\ \text { (3 terms) }\end{array} & \begin{array}{l}\text { \%difference } \\ \text { (5 terms) }\end{array} \\ 1 & 2 & 1 & 2 & 0.5 & -5 & -7 \\ 0 & 2 & 0 & 2 & 22 & -17 & -30 \\ 1 & 2 & 2 & 1 & -5 & 1 & 3 \\ 0 & 2 & 2 & 0 & -67 & 16 & 44 \\ 10 & 11 & 10 & 11 & -0.5 & -0.6 & -0.6 \\ 10 & 11 & 10 & 11 & -0.1 & 0.02 & 0.06\end{array}$

Large differences between the methods of multiplying together the two sloping lines are obtained when the spread between beginning and ending values is large compared with the minimum value in the range (i.e., when the difference is $100 \%$ of the spread). The averaging procedure will work well for those cases with small spreads and minimum values much removed from zero. Values near zero will probably have little influence on the total dosage. If experience shows this to be a weak point in the calculations, the matter will be revisited. Otherwise, it is important to use algorithms which make the computer programs as efficient as possible.

The filter spectra are similarly averaged to give the $\%$ transmission, $f$. The transmitted radiation is then

$I_{f}=f I_{0} / 100$

The specimen UV absorbance spectra are also averaged. The absorbance is defined as $A$ in the equation

transmitted radiation $\mathrm{I}_{\mathrm{t}}=\mathrm{I}_{\mathrm{f}} 10^{-\mathrm{A}}$.

The radiation absorbed by the specimen on the average per unit time is given by

$I_{f}-I_{t}=I_{f}\left(1-10^{-A}\right)=f I_{0} / 100\left(1-10^{-A}\right)$.

The dosage of the specimen during the time slice is $\mathrm{f} \mathrm{I}_{0} / 100\left(1-10^{-\mathrm{A}}\right) \Delta \mathrm{t}$, where $\Delta t$ is the duration of the time slice in seconds. All these quantities are now known. The dosage after each time slice is the sum of all dosages up to that point. In the database tables used up to now, $\mathrm{I}_{0}$ has been in $\mathrm{mJ} / \mathrm{m}^{2}$ and $\Delta \mathrm{t}$ has been in kiloseconds, which gives the dosage in Joules $/ \mathrm{m}^{2}$.

The following figures show the lamp and filter spectra as found by the program in the database tables. It is clear that 
1) the lamp changed somewhat during the exposure and that neglecting these changes is not good experimental practice, and

2) the filter was a narrow band-pass filter centered at about $300 \mathrm{~nm}$.

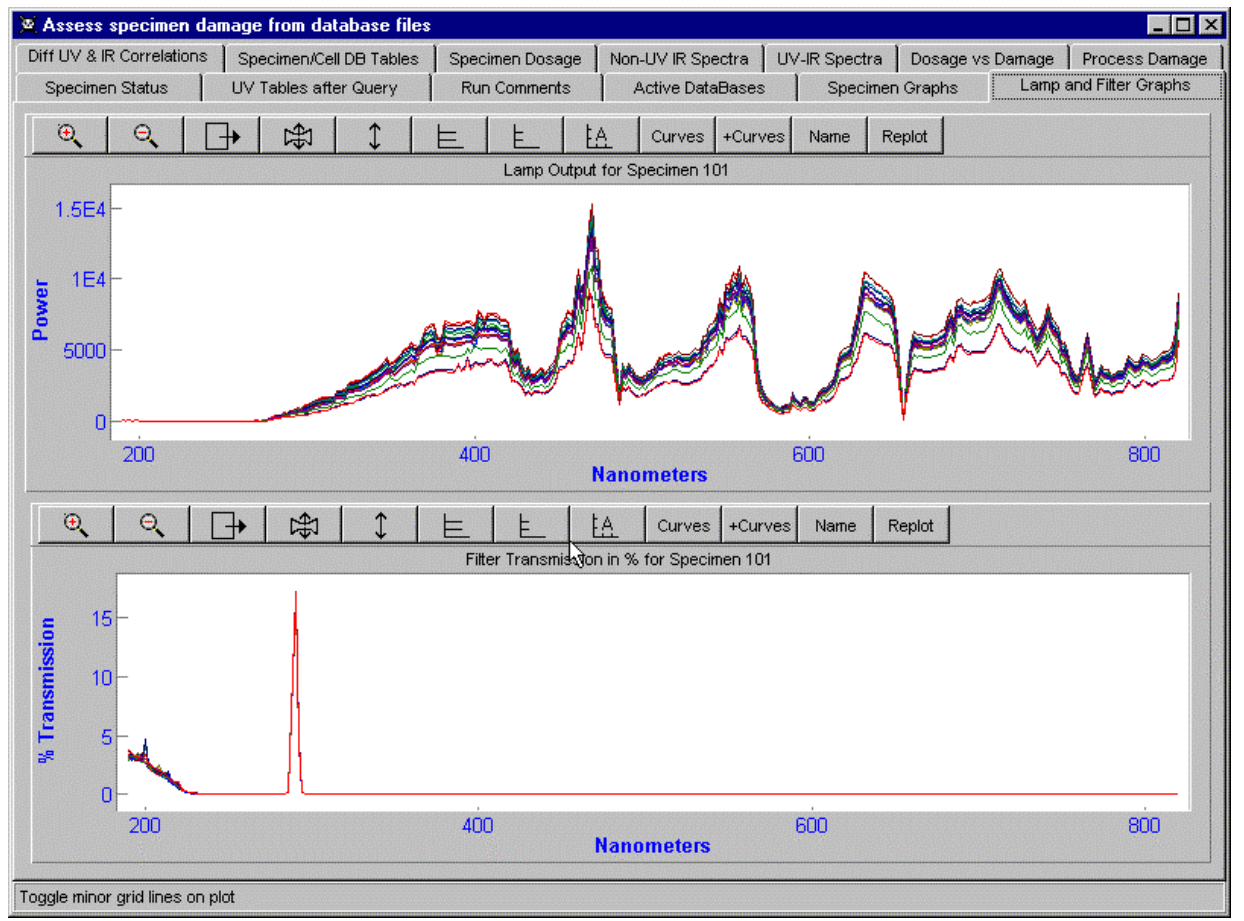

The plots have a series of buttons over the top of each plot. The uses of these buttons will be described in detail later, but for now it will suffice to point out that the plots can be zoomed. The zoomed versions of the lamp and filter spectra are shown below. The variation in the lamp intensity in this particular exposure (where the filter has its band pass) covers a range of about $100 \%$ of the smallest value. The filter was very stable. Note that the useful part of the lamp radiation was in the tail of the lamp spectrum and was very weak. Almost all of the lamp output was not used in this particular exposure, but other simultaneous exposures used other filters with band-passes in the other regions of the lamp output. Note also that the energy of radiation varies inversely with wavelength and the most destructive region for polymeric materials is typically in the range below $350 \mathrm{~nm}$ (for radiation to do damage, the polymer must have a chromophore which absorbs in that region). Sunlight begins at about 290 to $295 \mathrm{~nm}$. The lamp spectra have been transformed into $\mathrm{mW} / \mathrm{m}^{2}$ using the values in the Lamp Power table. Representing elapsed time in kiloseconds gives dosage in $\mathrm{J} / \mathrm{m}^{2}$. 


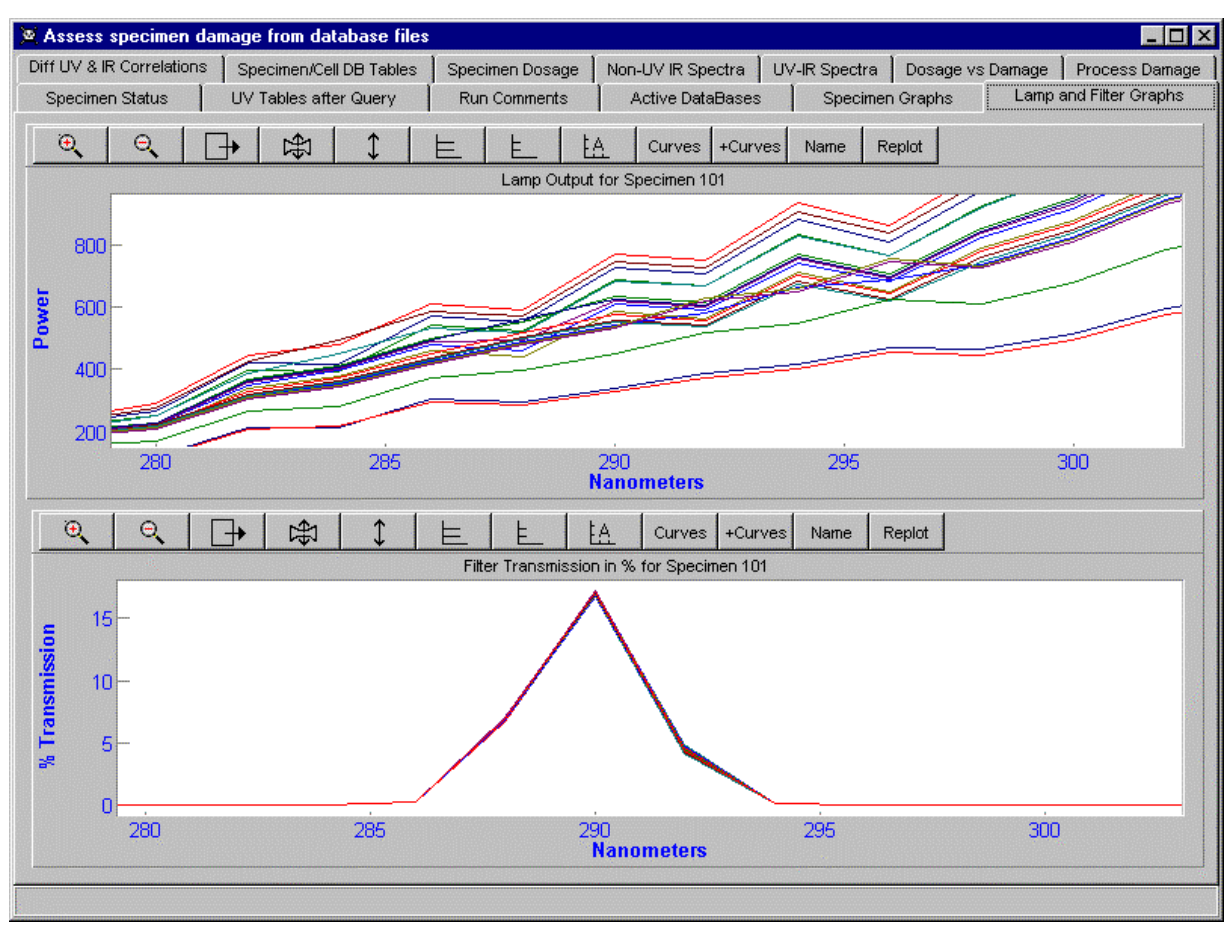

Note also that the filter did not pass more than $20 \%$ of the incident radiation and that only over a very narrow wavelength range. The profile of the filter band pass was measured fairly well but any changes in spectral resolution should move in the direction of finer steps in wavelength, not coarser steps. Not much radiation was produced by the lamp in this range and not much of the radiation passed through the filter - if not much degradation is seen in the specimen although the specimen UV absorbance is appreciable, this could be the reason. Such conclusions are clear and easily made when the data are conveniently and rapidly sorted, extracted and displayed by the computer. This allows the user to make very rapid progress in assessing the reasonableness of the results (it is always a good idea to check if the results make sense). In fact, it is highly probable that not much damage occurred unless the material was very sensitive to the radiation or it was exposed for a very long time.

Most of the lamp spectra are close in absorbance to their neighbors. There are two jumps but they are from the equivalent of 1 to 2 and even they are probably fairly well estimated by the averaging method. 


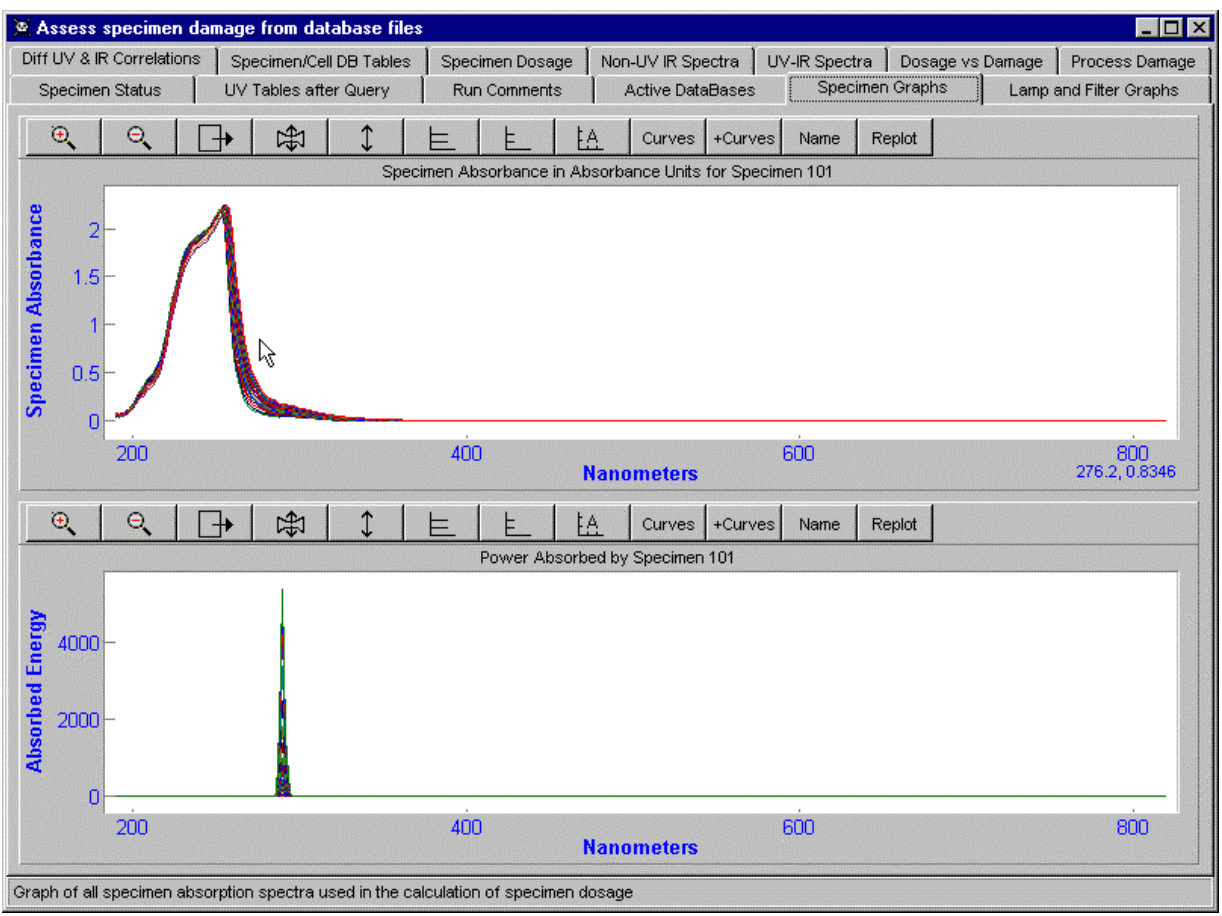

The specimen UV absorbance spectra are plotted on the "Specimen Graphs" page. The top plot is the series of specimen UV absorbances. Clearly, there was some change in the specimen, presumably as a result of damage. The bottom plot shows the dosage of the specimen - the amount of radiation remaining in the specimen.

Zooming these two plot gives the following display. The changes in the spectra seem to be regular and again the averaging method probably works well. (The colors of the curves repeat every 8 curves. The first 3 - which are easily described - are red, blue and green.)

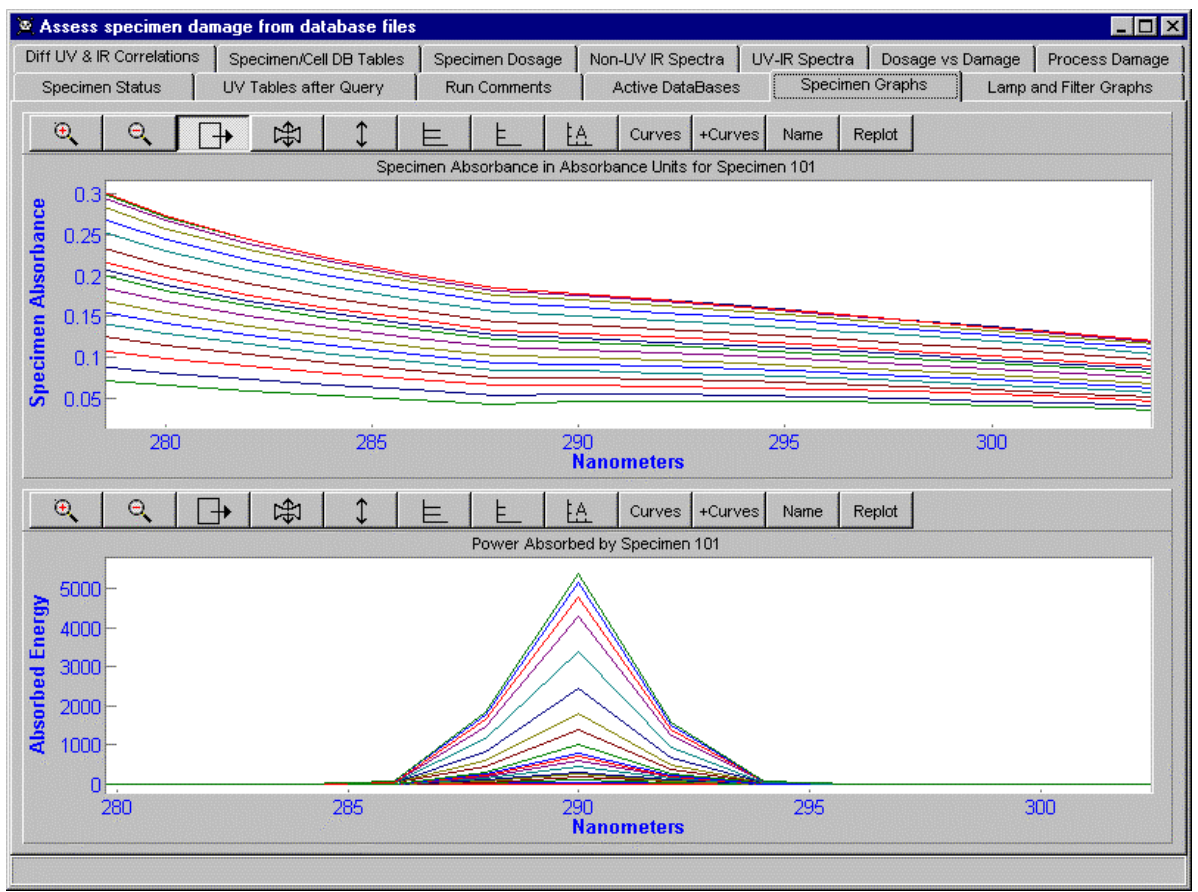


The dosage is in units of $\mathrm{mW} / \mathrm{m}^{2}{ }^{*}$ kiloseconds, which is $\mathrm{J} / \mathrm{m}^{2}$.

We now turn to the damage spectra. There, it is necessary to be able to find automatically which specimen is the control so that the changes in the control specimen can be subtracted from the changes in the exposed specimen. Typically, the control specimen is the non-UV specimen exposed to the same temperature and humidity as the specimen which received the UV exposure, but it can be selected by the user to be any of the specimens exposed at that temperature and humidity. The numbering system we use fits this requirement very well. The program expects that specimens exposed to a particular temperature and humidity have the same "hundreds" in their specimen number. The units (up to 10) specify the filter and 11 means no UV exposure. Thus, if the program is processing specimen 501, it merely has to find data for specimen 511 to know what happens in the absence of UV.

The first set of plots below show the damage spectra for the non-UV specimen. In this investigation, these are IR spectra. The top plot shows the spectra as read from the database, except that they may have been zeroed and scaled to correct for ablation if those options were specified on the "Process Damage" page. The bottom plot shows the difference spectra from the first spectrum, which was taken before the exposures were begun. Changes to the specimen during the exposure (even to no UV) are very evident in the difference plot. These are the "dark" reactions. Absorptions which grow with time denote chemical groups which are being produced; those which decrease with exposure are from chemical groups which are being consumed. The user can see how the various absorptions are related to one another by calculating the correlations - merely by setting the check box on the "Process Damage" page so that mouse clicks will be interpreted as denoting places for which to calculate the correlation with all other parts of the damage spectra and then clicking on one of the plots (presumably the difference plots will be more sensitive to changes occurring during the exposures). The resultant estimates of intra-

spectral correlations are plotted on the

Diff UV \& IR Correlations

page.

The damage spectra were zeroed where the short dotted vertical lines are shown in the top plots. 


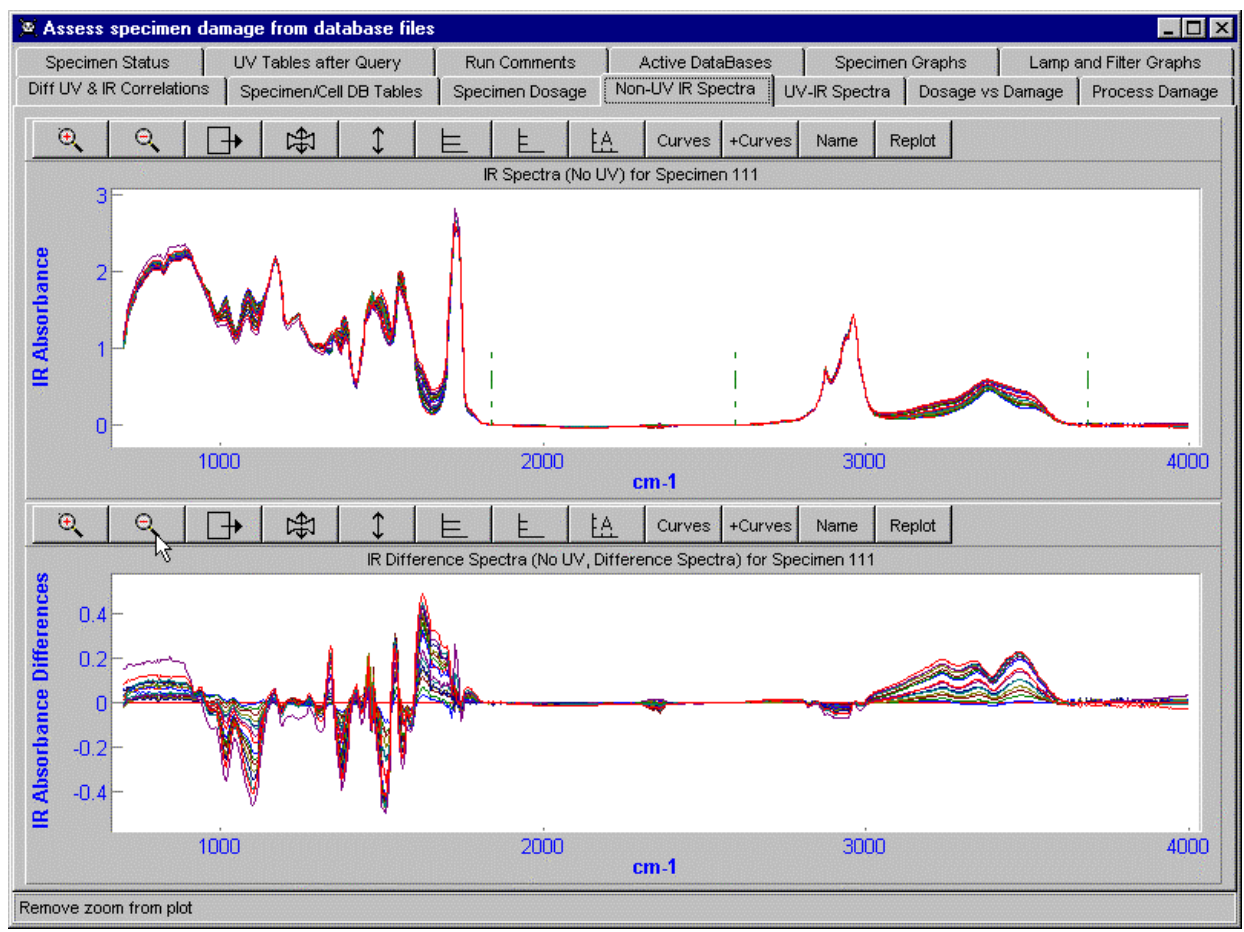

The corresponding damage plots for the UV-exposed specimen are shown in the next figure. The top plot contains the original spectra, except that they may have been corrected for zero, specimen thickness and ablation if those options were specified on the "Process Damage" page. The bottom plot contains "double" difference spectra. First, the difference from the first UV-exposed specimen spectrum has been calculated. This spectrum is taken before the specimen is exposed at all so the changes from it are those occurring during UV exposure. Then, if the option was specified on the "Process Damage" page, the control (usually the non-UV) spectra were subtracted. This removes the effect of the dark reactions. Although the spectra from a particular specimen are (in the current way of doing the experiments) from the same film spin cast on a large calcium fluoride plate, the parts of the film from which the spectra were taken may have slightly difference thicknesses. If the appropriate option was checked on the "Process Damage" page, the spectra will be scaled to constant specimen thickness before they are subtracted. This will also remove any effect of ablation.

By comparing the difference IR spectra from specimens not exposed to UV, where the maximum absorbance in the region 1600 to $1700 \mathrm{~cm}^{-1}$ is about 0.4 , with the UV-exposed, dark-reaction-subtracted spectra, where the maximum absorbance in the same region is about 0.08 , we see that the photolytic component of damage seems to be about $0.08 / 0.4=20 \%$ of the dark reaction (hydrolysis) for this material under these conditions. 


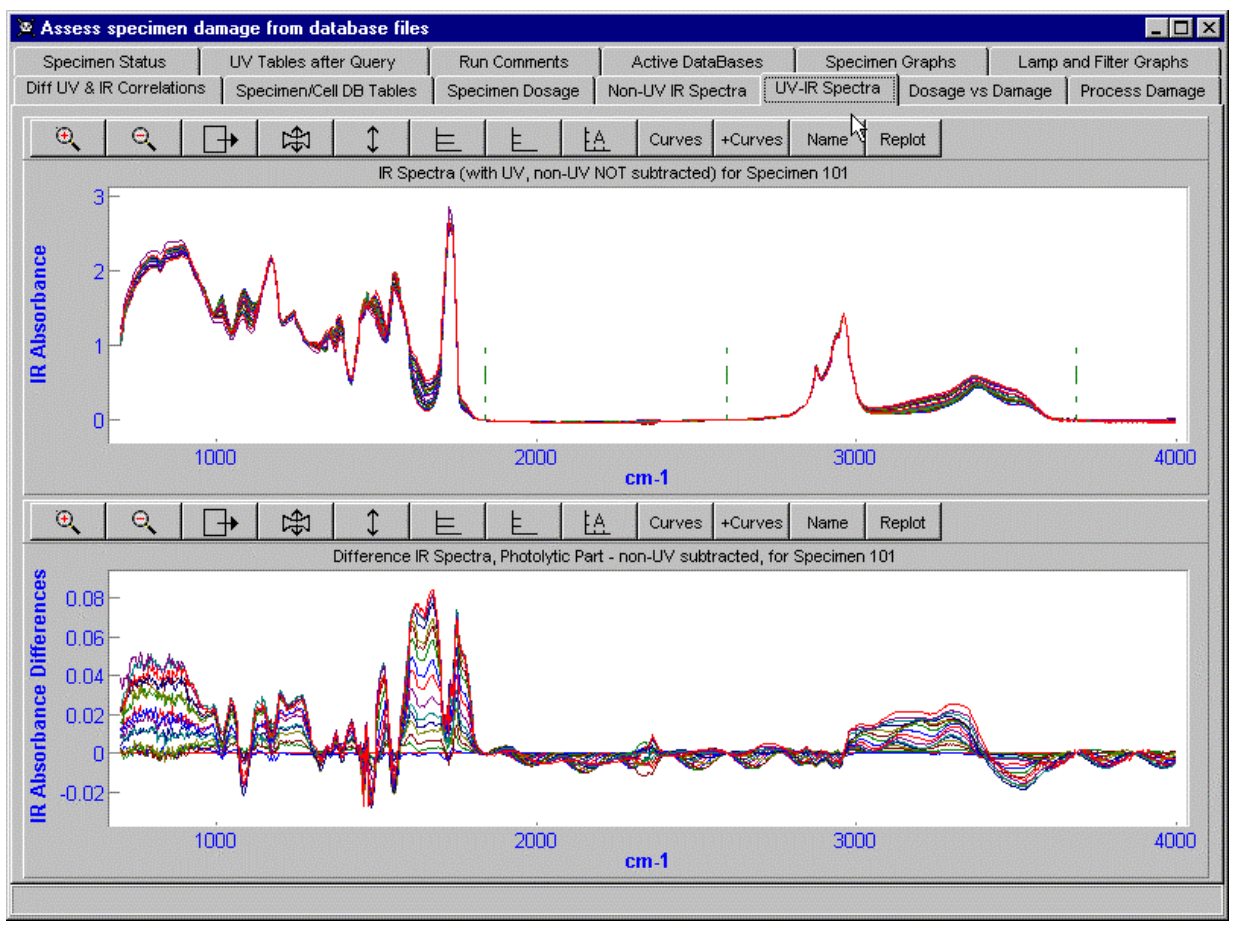

It may be necessary to measure or allow for ablation. After the program has read in the damage spectra, it calculates the apparent ablation for both the dark reaction, called control in the following figure, and for the UV degradation. The absorbance at the ablation site (specified on the "Process Damage" page) is used to estimate the sample thickness and hence the ablation.

$$
\begin{aligned}
& \text { Operate on Damage Plots } \\
& \text { Estimate specimen thicknessiablation in IR at } \quad 2960.00 \\
& \Gamma \text { If checked, click on IR will select new place to use to estimate ablation }
\end{aligned}
$$

In the example below, there seems to have been an error of about $1 \%$ in the IR spectra, judging from the apparent ablation in the dark. Maybe some ablation in the UV exposed specimen occurred although the effect was not very marked and was not much bigger than the apparent error. The ablation window pops up each time a set of IR spectra are read in and may be removed by clicking on the close box (the $\mathrm{x}$ in the upper right hand corner) or simply ignored by clicking on some part of the main program window, in which case the ablation window disappears behind the main program window. The ablation window may be made visible

again by clicking on the Show Ablation Details of the program. 


\begin{tabular}{|c|c|c|c|c|c|}
\hline \multicolumn{4}{|c|}{ Ablation Details } & \multicolumn{2}{|c|}{$-\square \times$} \\
\hline \\
\hline & & & & & \\
\hline \multicolumn{6}{|c|}{ Specimen ID $\quad$ Dupont } \\
\hline \multicolumn{6}{|c|}{ Filter Max $\quad 290.00$} \\
\hline \multirow{2}{*}{\multicolumn{6}{|c|}{$\begin{array}{l}\text { Wavelength Range in UV } 280 \text { to } 301 \mathrm{~nm} \\
\text { Temperature }=60.0, \mathrm{RH}=20.7 \%\end{array}$}} \\
\hline & & & & & \\
\hline \multicolumn{4}{|c|}{ From the IR Spectra } & \multicolumn{2}{|c|}{ From Photolysis } \\
\hline $\begin{array}{l}\text { A Control } \\
\text { Days }\end{array}$ & $\begin{array}{l}\text { Ablation } \\
\text { of Control }\end{array}$ & $\begin{array}{c}\text { UV } \\
\text { Ablation }\end{array}$ & $\begin{array}{l}\text { Days of UV } \\
\text { Exposure }\end{array}$ & \multicolumn{2}{|c|}{$\begin{array}{l}\text { Actual Exposure } \\
\text { Days }\end{array}$} \\
\hline 0.0000 & 1.000 & 1.000 & 0.0000 & 0.0000 & $\Delta$ \\
\hline 0.1667 & 1.012 & 1.012 & 0.1667 & 0.8264 & - \\
\hline 1.0000 & 1.006 & 1.005 & 1.0000 & 1.6562 & \\
\hline 2.0000 & 0.995 & 0.993 & 2.0000 & 2.5069 & \\
\hline 3.0000 & 0.995 & 0.991 & 3.0000 & 3.3714 & \\
\hline 4.0000 & 0.999 & 0.992 & 4.0000 & 4.2325 & \\
\hline 5.0000 & 0.995 & 0.988 & 5.0000 & 5.9339 & \\
\hline 7.0000 & 0.994 & 0.984 & 7.0000 & 7.5555 & \\
\hline 10.0625 & 0.982 & 0.972 & 10.0625 & 8.6388 & \\
\hline 14.0000 & 0.991 & 0.981 & 14.0000 & 9.4465 & \\
\hline tanama & anat & $a m m$ & tanama & tharm & च \\
\hline
\end{tabular}

The change in specimen UV absorbance with increasing exposure (see the top plot in the next figure) is provided automatically by the program as part of the philosophy that the information is extremely important in estimating the dosage because it enters the equation as an exponent of 10. Further, its trend with exposure may be helpful in providing understanding of the UV degradation process. For example, probably not all the apparent dosage is effective dosage because some is harmlessly absorbed by degradation products.

In the UV degradation of this specimen, some UV absorption was lost at about 200-240 nm (perhaps ester groups, carbonyl groups, etc.) and a significant amount of new absorbance was generated in the region of about $240-320 \mathrm{~nm}$. 


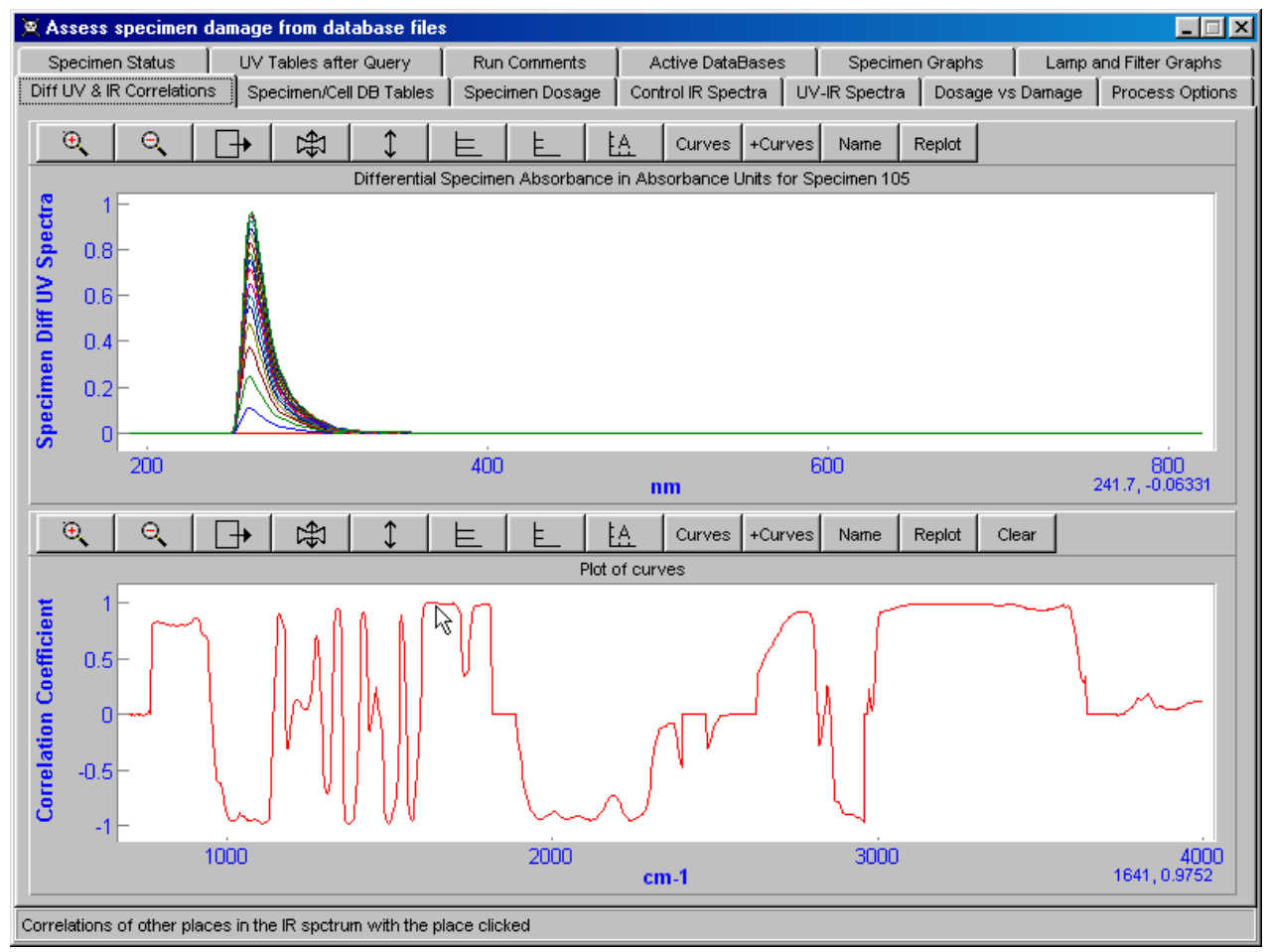

The lower plot in the above figure is the correlation of about $1640 \mathrm{~cm}^{-1}$ in the damage IR spectrum with the rest of the spectrum. The place clicked is not mentioned in the caption because it is possible to click several times, each time putting a new curve in the plot. A value of +1 means that that region of the IR spectrum is well-correlated with the place clicked and may well be another absorption from the same chemical group. A correlation of -1 means very good correlation but with an opposite trend with exposure. This may mean that the group was consumed to produce the group that appeared, although of course that is not necessarily so.

\section{At this point, the program has finished the sequence started by clicking on the button "Process Specimens Manually".}

Further operations require directions from the user. The user can now do what the automatic processing would do. The first thing is to integrate the dosage curve. The wavelength limits over which to integrate may be specified on the "Specimen Dosage" page (see the next figure). When processing automatically, the program estimates these limits from the filter maximum and half width information in the Filter IDs database table. 


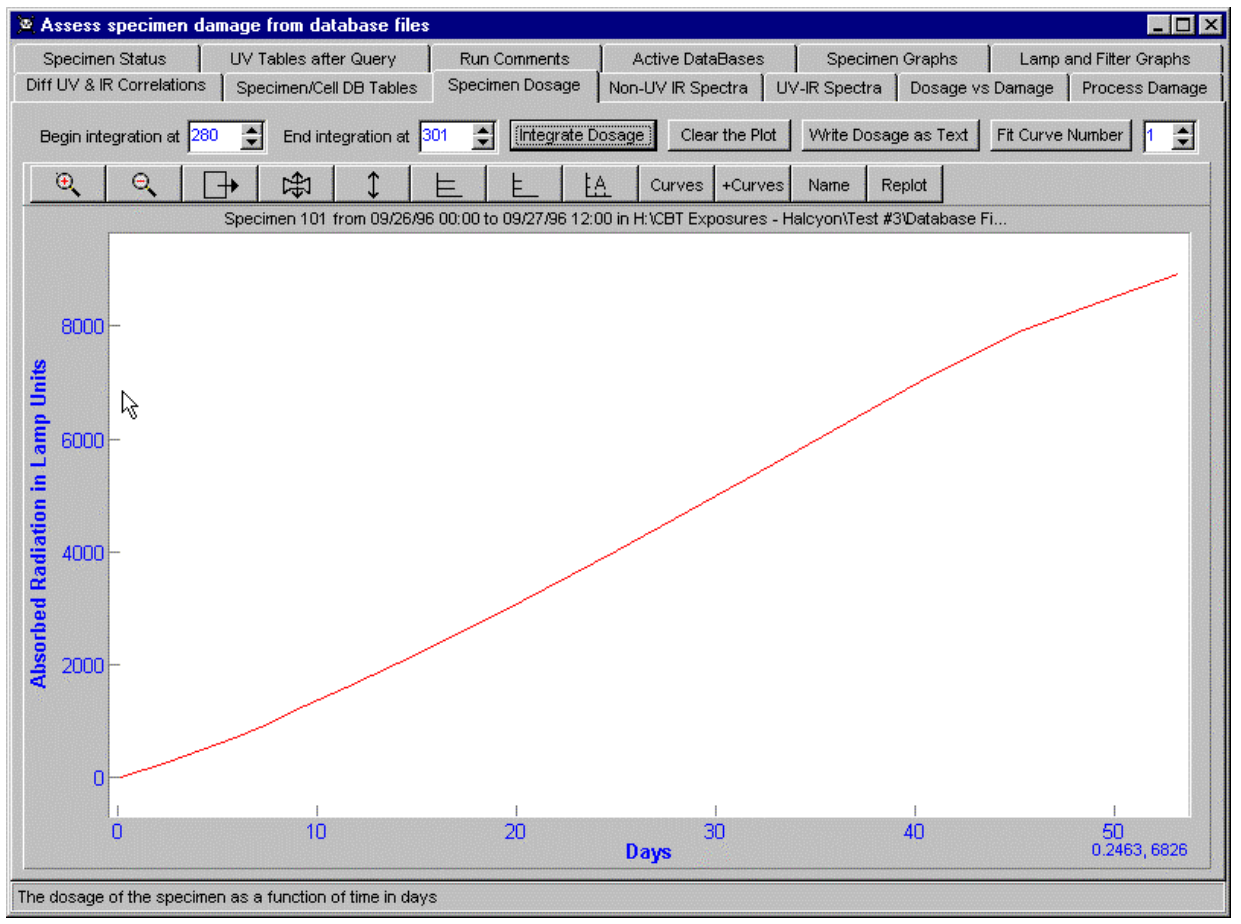

After the filter width limits have been chosen, clicking on the "Integrate Dosage" button puts a new curve in the dosage/time plot. If more than one curve is in the plot, the curve to use to estimate dosage as a function of time can be set using the spin button "Fit Curve Number" in the top right hand corner of the page. The dosage/time curve must be filled in before damage sites can be selected in the damage plots.

Returning to the damage plots, we probably want to click on the plot of difference IR spectra from specimens exposed to UV-visible radiation at the bottom of the "UV-IR Spectra" page (see next figure). 


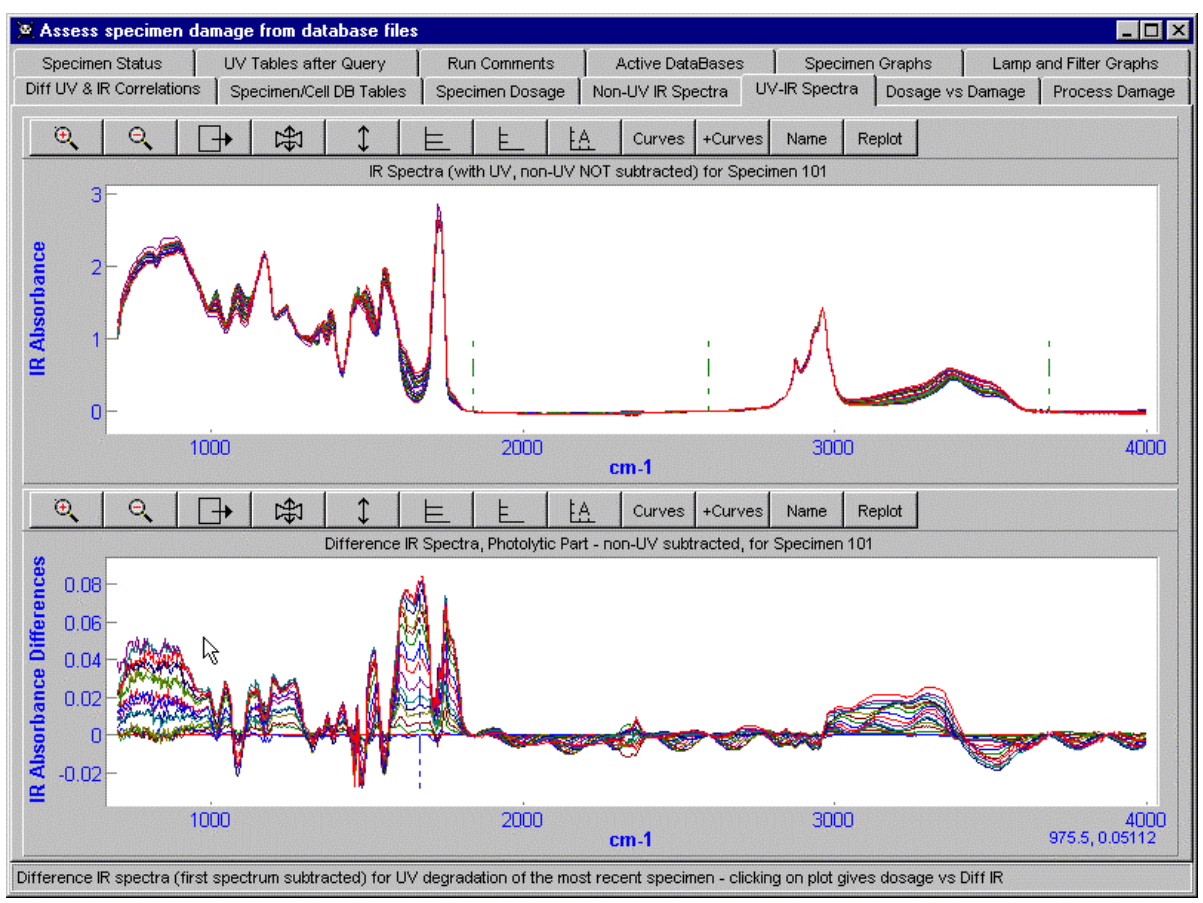

If the mouse is right-clicked on the difference UV-exposed specimens plot (or the top IR plot), the IR absorbances at the point clicked in each of the spectra are plotted as a function of time (the time at which each spectrum was taken) in the top plot on the "Dosage Vs Damage" page (see the next figure).

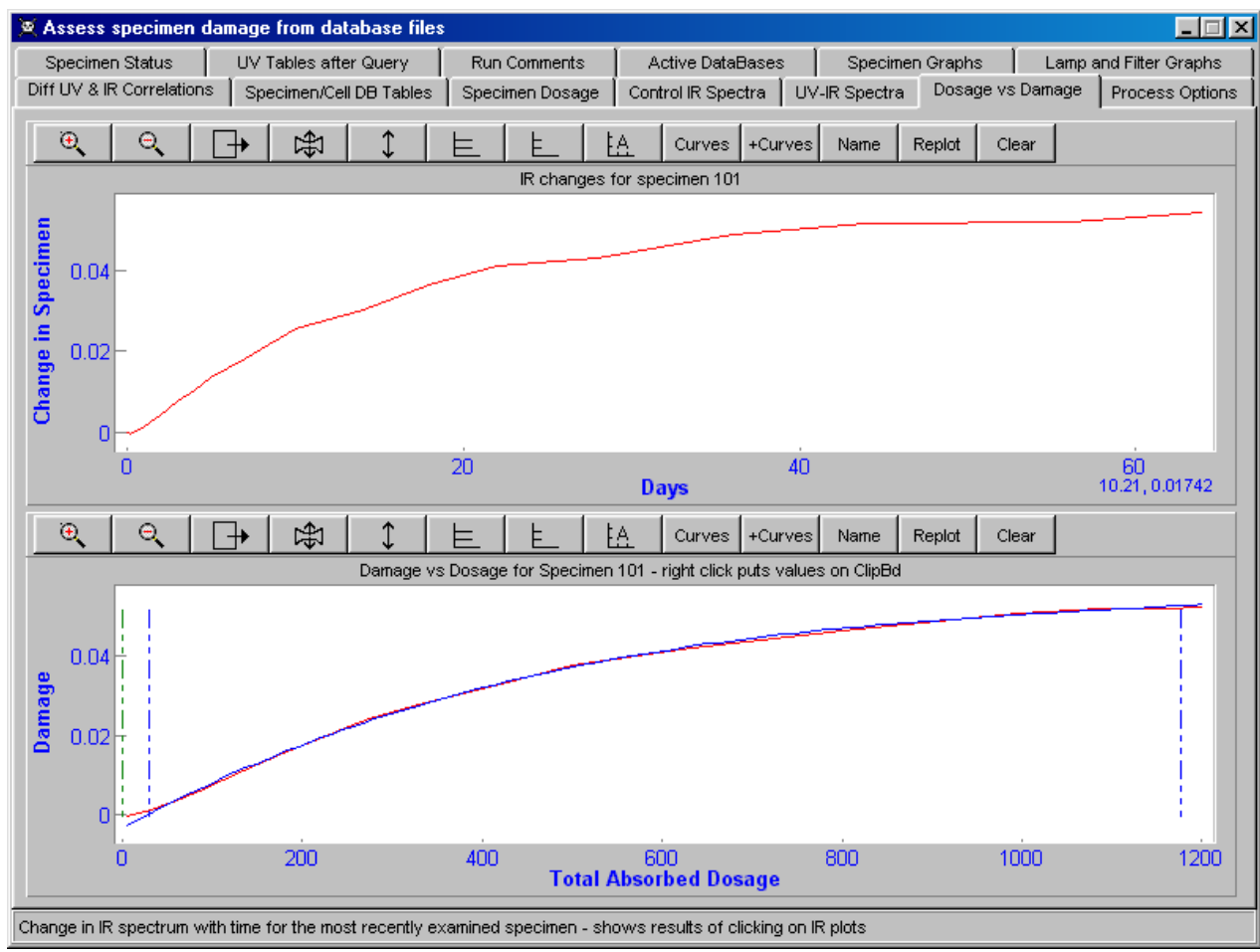

The program now has dosage vs. time and damage vs. time, so it can easily calculate damage vs. dosage, using the time axis as a common 
axis. This plot is at the bottom of the "Dosage vs. Damage" page and is the plot which allows the estimation of the quantum yield.

The shape of the damage/dosage curve is not known a priori so the program tries to fit a 4 degree polynomial to it. The result is shown as a blue curve in the lower plot in the above figure. The polynomial is actually plotted using the next available curve color in the plot, so the colors of the damage/dosage curve and the fitted curve will depend on how many curves are already in the plot. The various curves can be identified by first clicking on the "Names" button over the plot so the button is down, then right clicking near the curve of interest, when the program will give the name of the curve nearest the point clicked in a message box.

It is now necessary to calculate the slope of the damage/dosage curve the quantum yield - using the controls pictured below. As always, having the signal much greater than the noise simplifies the procedure.

However, the fit will not make sense if the curve is merely noise, or even if the curve is mostly noise. Various parameters have been provided on the "Process Damage" page to allow the user to limit the polynomial fitting and quantum yield estimation to regions of the damage/dosage curve where the signal is non-noisy.

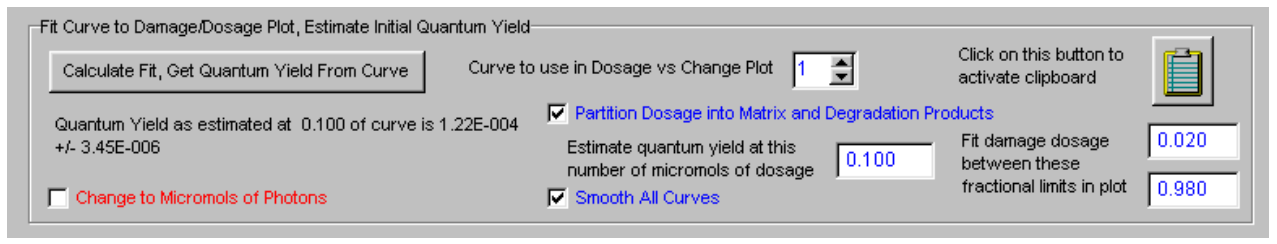

The "early" spectra are often closely spaced in time, when any differences between spectra are small. In some cases, the beginning of the damage/dosage plot is obviously not trustworthy - the signal is small and is not able to dominate the noise. Sometimes the later spectra seem to include some larger than expected deviations. For these reasons, it is possible to limit the region of the damage/dosage plot to which the polynomial will be fitted using fractional limits in the dosage axis (horizontal axis) of the plot.

$\begin{array}{ll}\text { Fit damage dosage } & 0.020 \\ \text { between these } & \\ \text { fractional limits in plot } & 0.980\end{array}$

The slope of the damage/dosage curve often falls off with increasing dosage and where to estimate a particular quantum yield is left to the user. It is presumably most consistent to estimate the quantum fairly early in the degradation process, before complications arise.

Estimate quantum yield at this number of micromols of dosage 
To calculate a quantum yield manually, choose the curve in the Damage/dosage plot to which the polynomial is to be fitted - the spin button is set to curve 1 in the example below. The other choices, such as fractional limits of the dosage axis over which to fit the polynomial must also be chosen. Typically, these are left "as is" for a series of calculations. Then click on the "Calculate Fit, Get Quantum Yield From Curve" button, shown below.

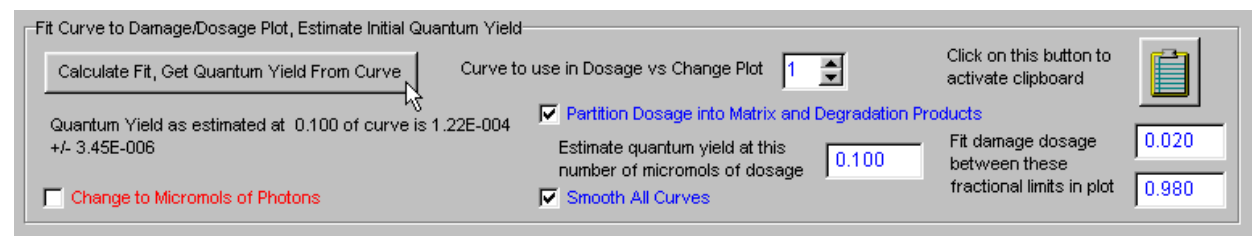

When the polynomial has been fitted (see the Damage versus Dosage plot in an earlier figure), essentially the entire variation of quantum yield with increasing dosage is encapsulated in the polynomial. The "initial quantum yield" calculated in this way and given after the calculation on the "Process Damage" page is an indication of the degradation per unit dose.

The next figure is from a different case than the Damage versus Dosage curve above. In particular, the quantum yield is negative because the chemical group is disappearing with increased dosage, whereas in the earlier example it was appearing with dosage. Quantum yields are, according to the strict definition of quantum yield, always positive because they are probabilities, but the negative sign has been kept here to show the group is disappearing. The standard deviation accompanying the quantum yield is that propagated from the errors obtaining in the least squares estimation of the coefficients of the polynomial.

\begin{tabular}{|c|c|c|}
\hline Calculate Fit, Get Quantum Yield From Curve & Curve to use in Dosage vs Change Plo & $1 \div$ \\
\hline \multirow{2}{*}{$\begin{array}{l}\text { Quantum Yield as estimated at } 0.100 \text { of curve is } \\
-4.92 \mathrm{E}-006+i-1.59 \mathrm{E}-006\end{array}$} & \multicolumn{2}{|c|}{ Г Partition Dosage into Matrix and Degr } \\
\hline & $\begin{array}{l}\text { Estimate quantum yield at } \\
\text { this fraction of dosage }\end{array}$ & 0.100 \\
\hline
\end{tabular}

Propagation of errors will be needed when specimen lifetimes are being predicted. The random errors in the damage/dosage relationship are propagated via the standard errors in the polynomial coefficients. Calculating these standard errors requires that determinants be evaluated. Various tests in the program ensure the program will survive while blindly trying to fit a polynomial to an unknown set of data. Attempts are made to keep only those coefficients which are above the noise. If any of these tests indicates that the fit is nonsense, the coefficients will be set to zero and a horizontal fitted line at zero on the vertical axis will ensue. 


\section{Adding Results to a Damage Database Table}

If results are being processed automatically, they are added to the damage database table specified on the "Active Databases" page of the program if the $\quad \Gamma$ Add To Damage DE if Auto Mode check box on the first
page of the program (Specimen Status) is checked.

If no damage table is currently assigned to the program, and the data in the spectra pass the various tests for low noise etc. on the Process Options page, a damage table must be assigned. The following dialog appears:

\begin{tabular}{|c|c|c|c|}
\hline Select Damage DB & & & \\
\hline 6 Database Files & $\Rightarrow$ t & $=\sqrt{\substack{0=0 \\
0=0 \\
0=0}}$ & \\
\hline Name & Size & Type & Modified \\
\hline 区] Cell ID.dbf & $4 \mathrm{~KB}$ & DBF File & 10/3/97 9:12AM \\
\hline 区] CellPositions.dbf & $13 \mathrm{~KB}$ & DBF File & $12 / 15 / 978: 20 \mathrm{AM}$ \\
\hline (ע] Copy of SampleSpectra.dbf & $84 K B$ & DBF File & 3/17/98 4:53 PM \\
\hline 区] Filter ID.dbf & $40 \mathrm{~KB}$ & DBF File & $2 / 9 / 986: 35 \mathrm{PM}$ \\
\hline 凶] FilterSpectra.dbf & $85 K B$ & DBF File & $10 / 3 / 979: 43 \mathrm{AM}$ \\
\hline 区] IRSpectra.dbf & $84 K B$ & DBF File & 10/6/97 9:35 AM \\
\hline 凶] Lamp ID.dbf & $1 \mathrm{~KB}$ & DBF File & $6 / 18 / 972: 09 \mathrm{PM}$ \\
\hline 区] Lamplntensity.dbf & $95 \mathrm{~KB}$ & DBF File & 7/30/97 2:02 PM \\
\hline 区] LAMPPOWEASBF & $29 K B$ & DBF File & 1/9/98 11:00 AM \\
\hline 区] LampsOnOff.dbf & $3 \mathrm{~KB}$ & DBF File & 8/21/97 10:04 AM \\
\hline 函POS2LAMPS.DBF & $4 \mathrm{~KB}$ & DBF File & 10/3/97 9:44 AM \\
\hline 区] SampleSpectra.dbf & $84 K B$ & DBF File & $3 / 17 / 987: 53 \mathrm{PM}$ \\
\hline 区] SpecimenID.dbf & $60 \mathrm{~KB}$ & DBF File & 2/11/98 4:44 PM \\
\hline «] SpecimensCells.dbf & $4 \mathrm{~KB}$ & DBF File & $12 / 12 / 9712: 44 \mathrm{PM}$ \\
\hline 区 Supplier.dbf & $8 K B$ & DBF File & $4 / 22 / 977: 56 \mathrm{AM}$ \\
\hline File name: & & Qpen & \\
\hline Damage DB (.dbf) & $\nabla$ & Cancel & \\
\hline
\end{tabular}

Type the file name for the damage table in the File Name box at the bottom of the File Open dialog and click on the Open button, or click on Cancel to stop the process.

\begin{tabular}{|c|c|c|}
\hline File name: & DemoDamageldbf & Dpen \\
\hline Files of type: & Damage DB [“.dbf] & Cancel \\
\hline
\end{tabular}

The program will try to locate and assign the damage table. If it can not find the file, it will create a damage table. 
If the damage table has been assigned (for an existing file) or created successfully, damage results will be added to it as they are calculated.

If processing is manual, the results can be added to the damage table

using the

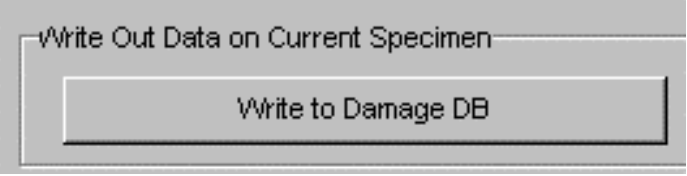

button on the Process Option page.

\section{Writing Damage/Dosage Curves to Text Files}

Clicking the Damage vs Dosage Data written toText File button

asks for a file name to use when writing the damage/dosage information to disk. Each curve in the plot is treated in turn. Each would have to have a different file name.

\section{Plot Control Buttons}

\section{The 13 Plot Buttons}

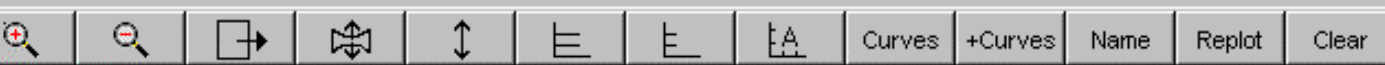

13 buttons over the plot frame provide an easy-to-use means of manually manipulating the plot. Each will now be demonstrated. The example is from chromatography, but the functions of the buttons are not dependent on the type of curve in the plot. Not all buttons are shown over each plot - it depends whether the user should be able to clear the plot or not and whether he should be able to manipulate the data in the curves. In the case of the various spectra, for example, the program requires the spectra to be available in the plot so no provision is made for the user to be able the clear the plot. 


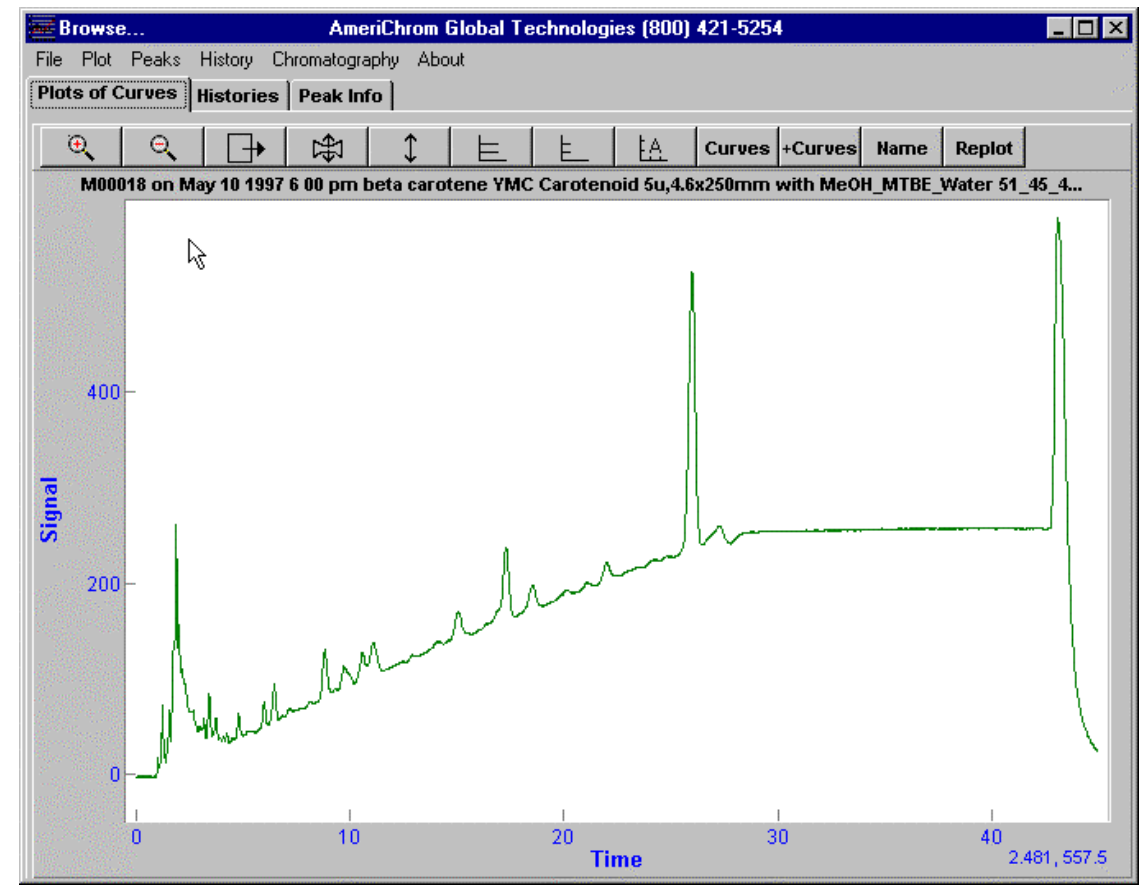

The first button is the zoom button. After the zoom button has been selected and is depressed, a rectangle can be drawn on the plot by dragging the mouse over the plot with the left mouse button down. When the mouse button is released, the contents of the rectangle will fill the screen, as shown in the next two figures.

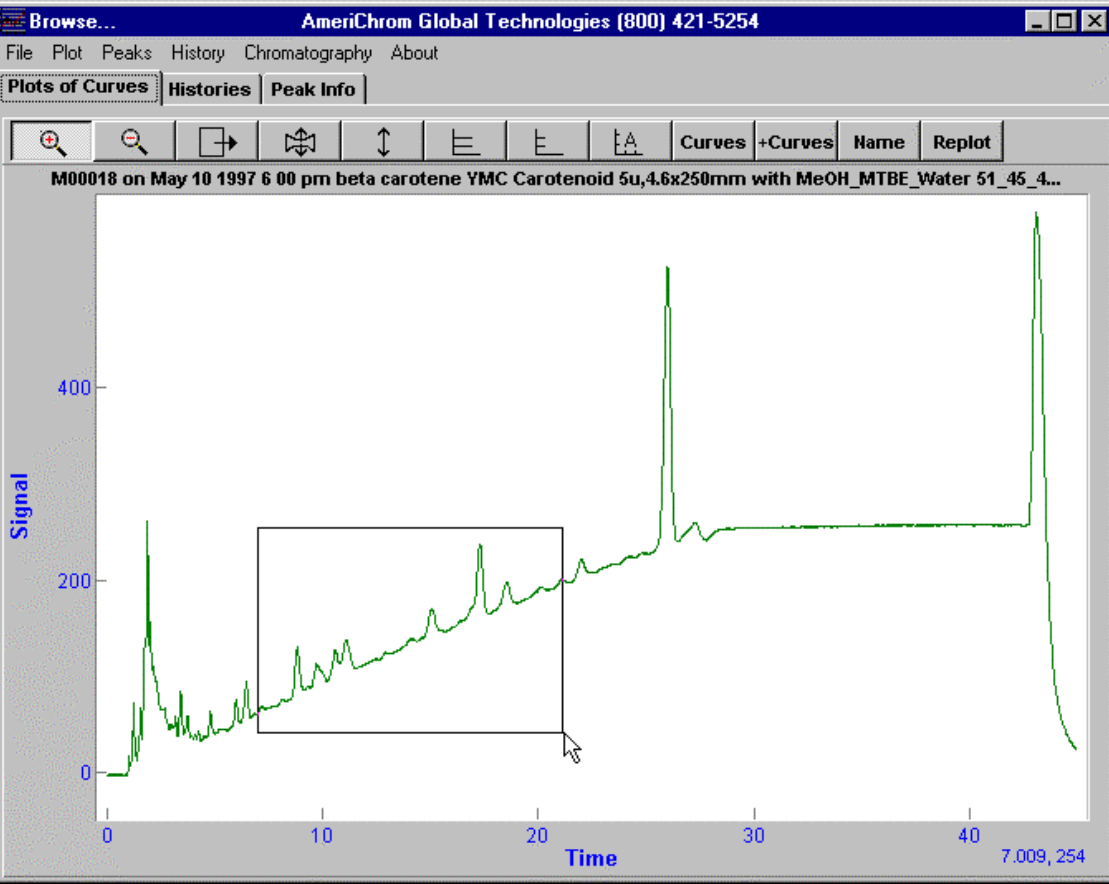




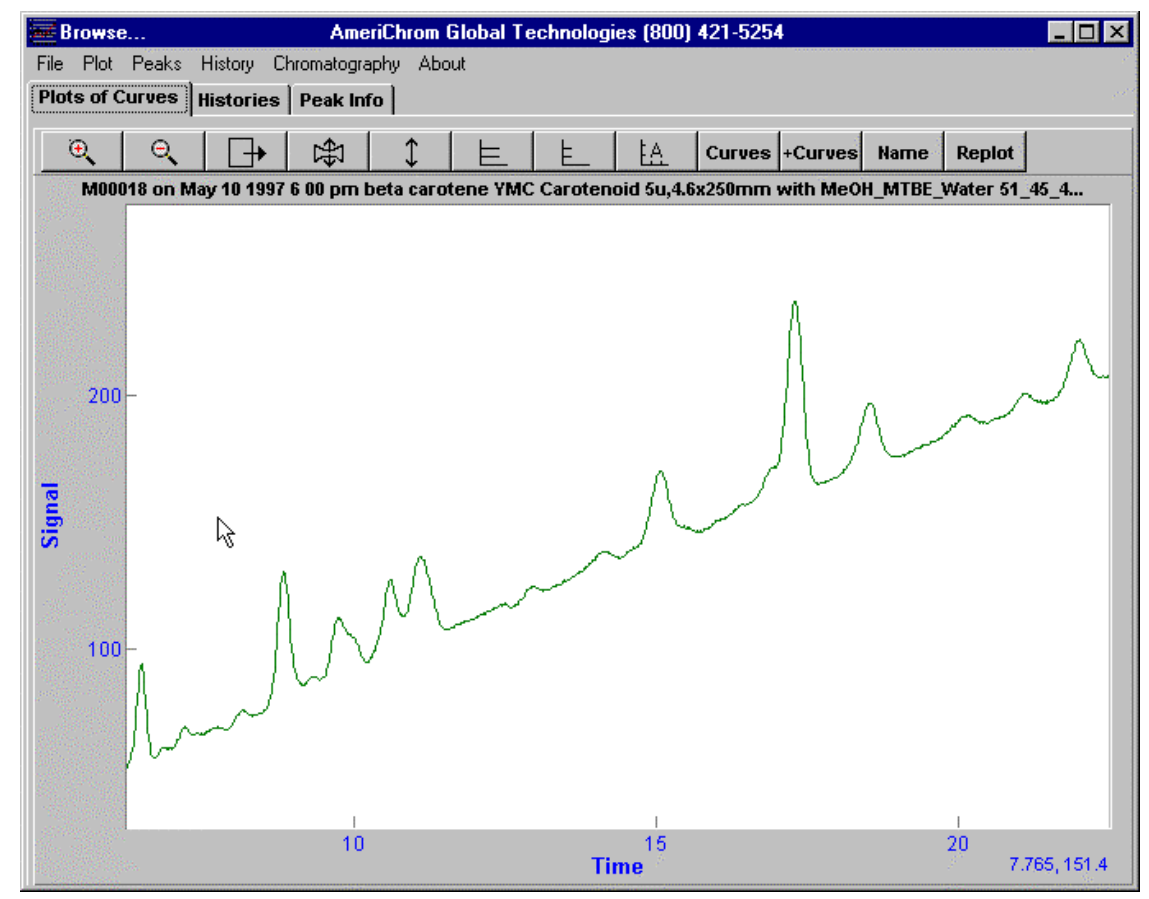

The zoom is removed by clicking on the second button, indicated in the figure below:

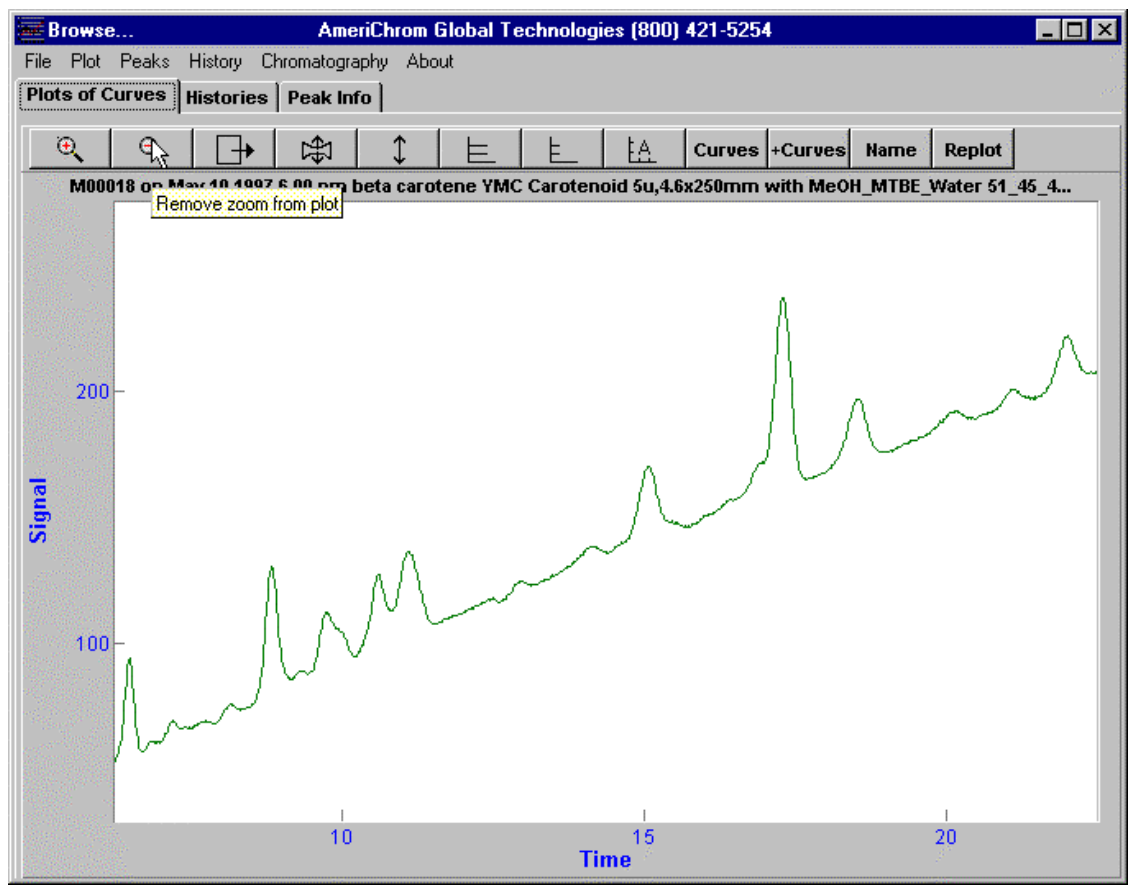

When the plot has been zoomed, the plot contents can be dragged around by first clicking on the third plot button, as indicated below, then dragging the plot contents with the left mouse button depressed. In this way, new areas can be scrolled into the visible area from out of the 
picture, as shown in the next two figures. The numbers on the horizontal axis show that the curve has been dragged from left to right.
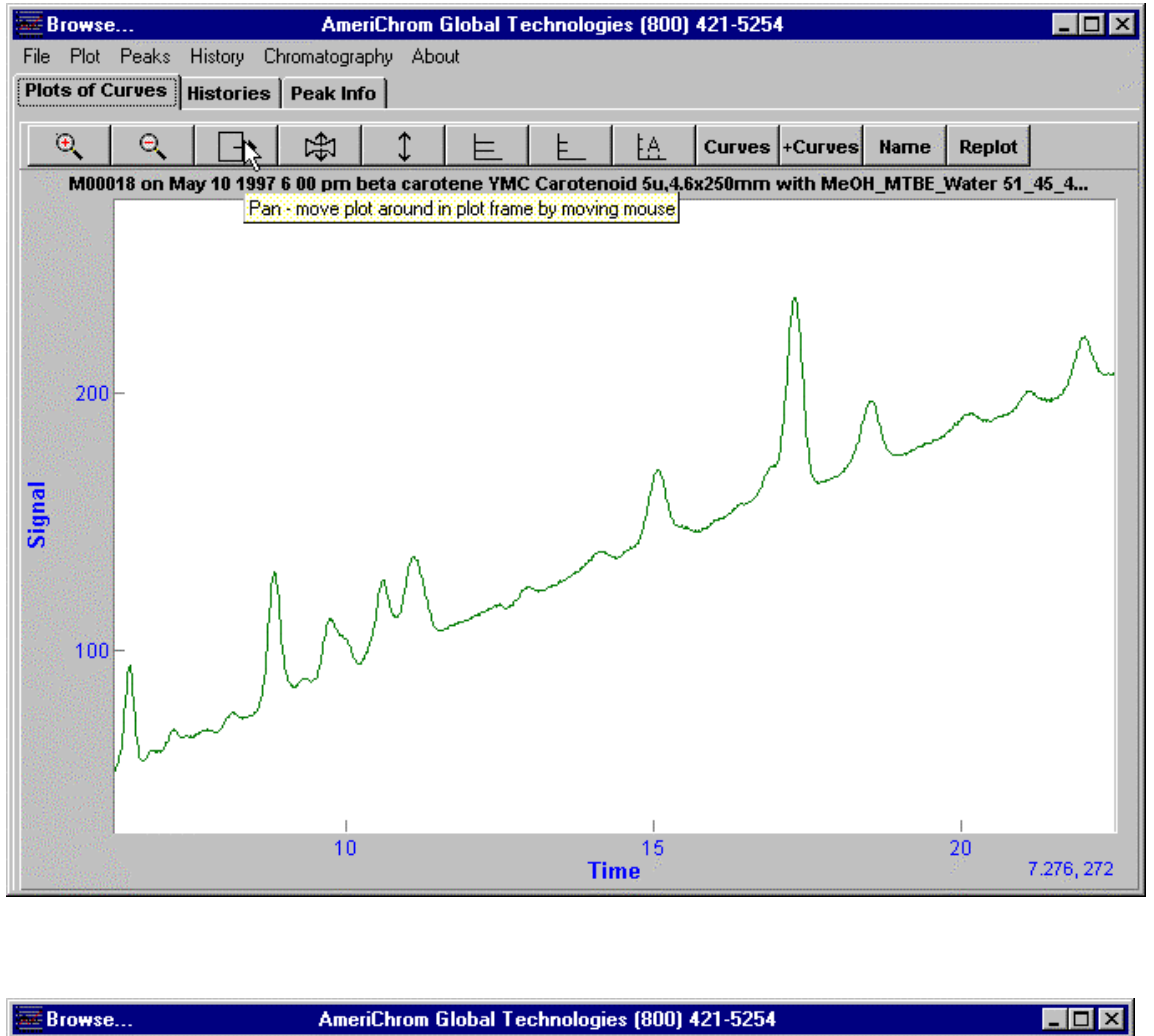

File Plot Peaks History Chromatography About

Plots of Curves | Histories |Peak Info |

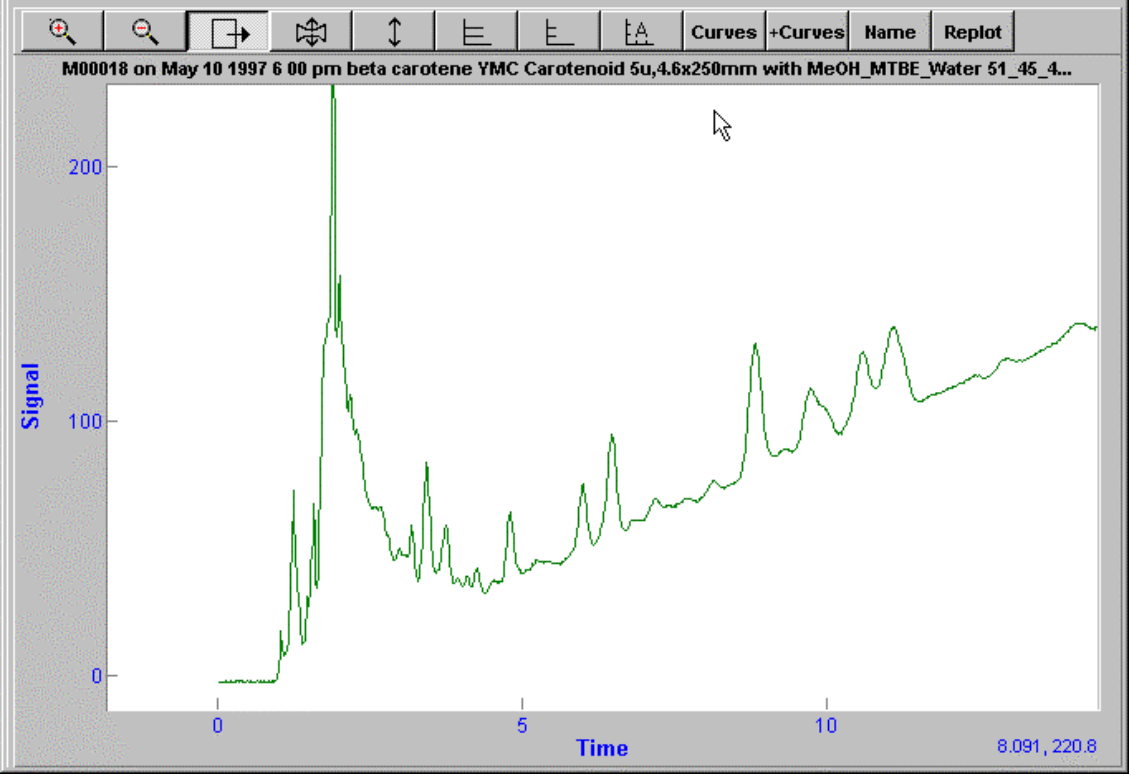


When the plot is not zoomed, everything in the plot is already visible on the screen, so no dragging is possible.

The plot contents can be stretched or compressed using the fourth button. First click on the fourth button with the left mouse button, then drag the plot contents with the left mouse button held down. Going horizontally left expands the plot horizontally, going horizontally right compresses it horizontally. Going up vertically expands the plot vertically, going down vertically compresses the plot vertically. Going diagonally produces mixtures of these modes.

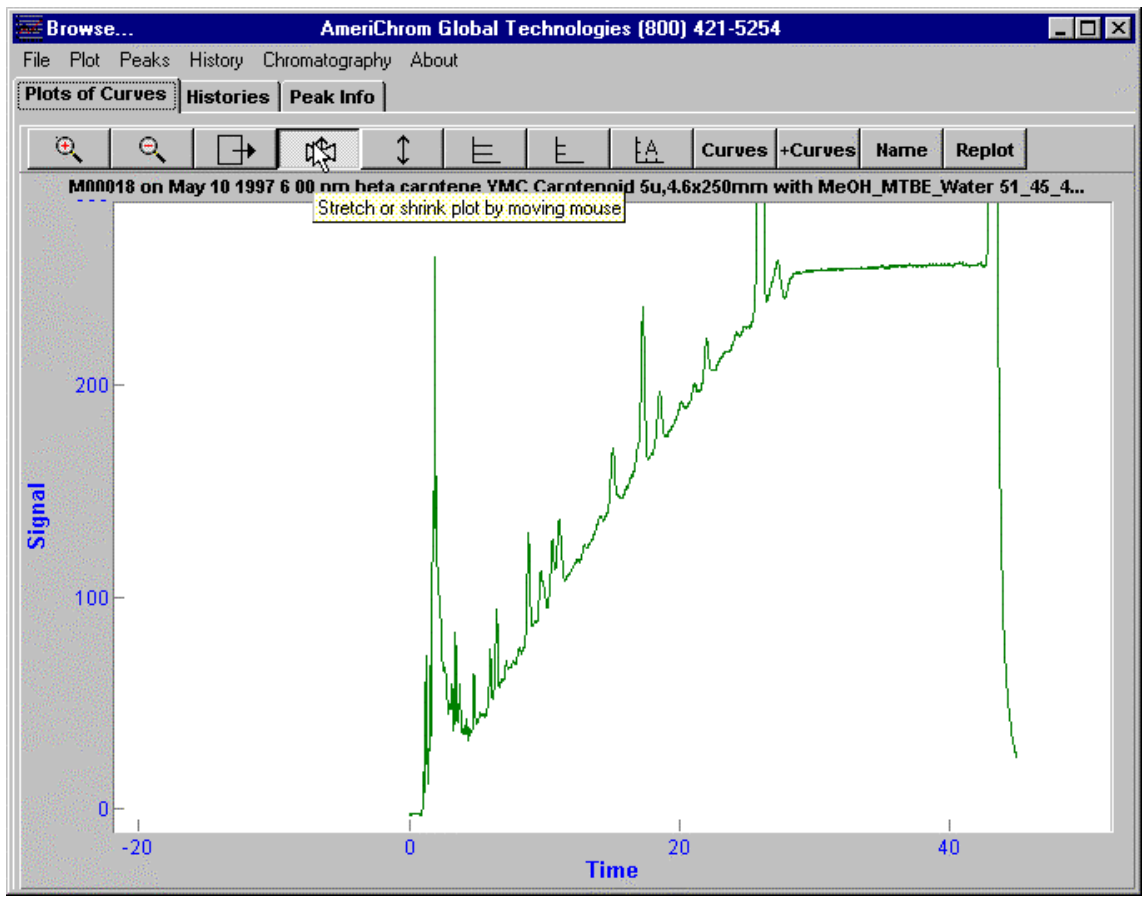

The fifth plot button leaves the plot as is in the horizontal direction but makes the $y$ data of the plot fit in the visible window, as shown below. 


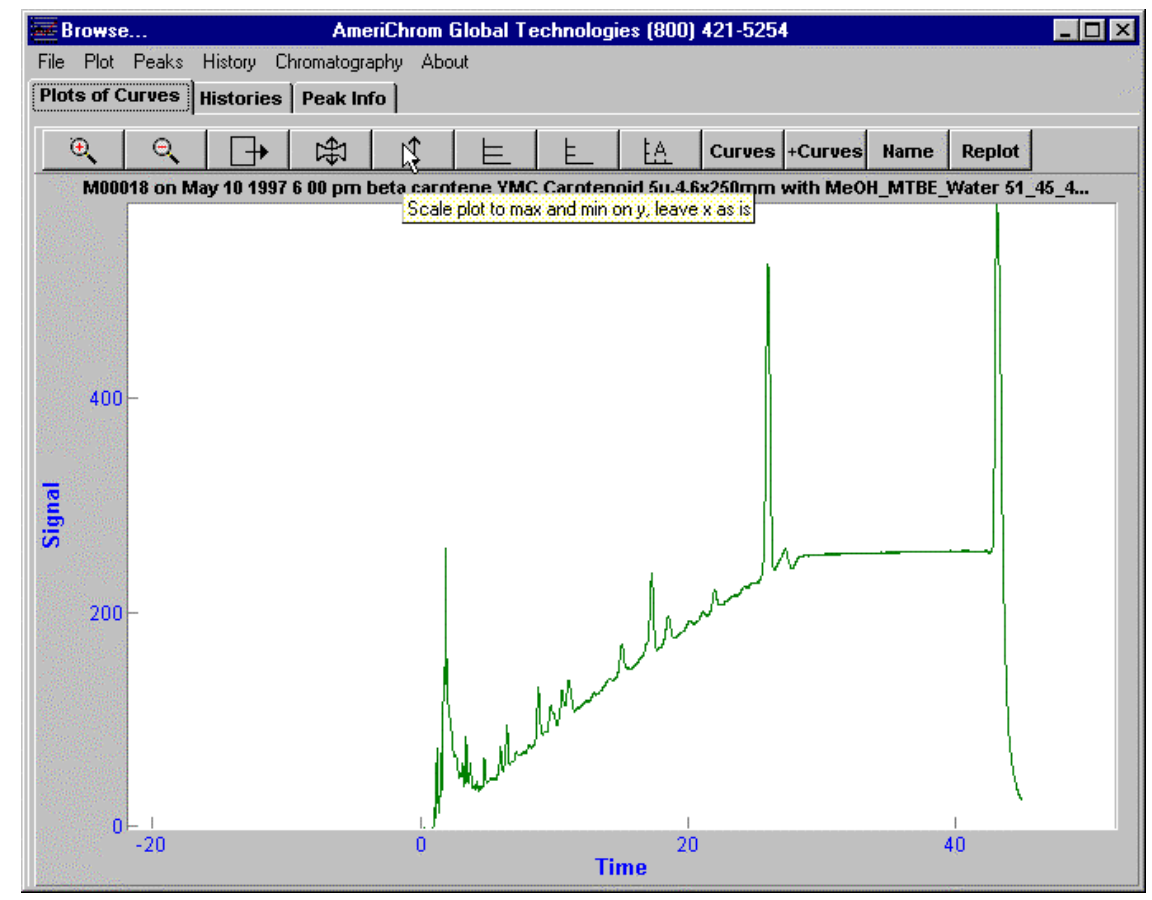

Two buttons draw grids on the plot. Clicking on the sixth plot button so it is depressed draws a coarse grid, and clicking on the seventh plot button draws a fine grid, as shown in the next two figures. The grids are removed by clicking on the depressed buttons so they move into the up position.

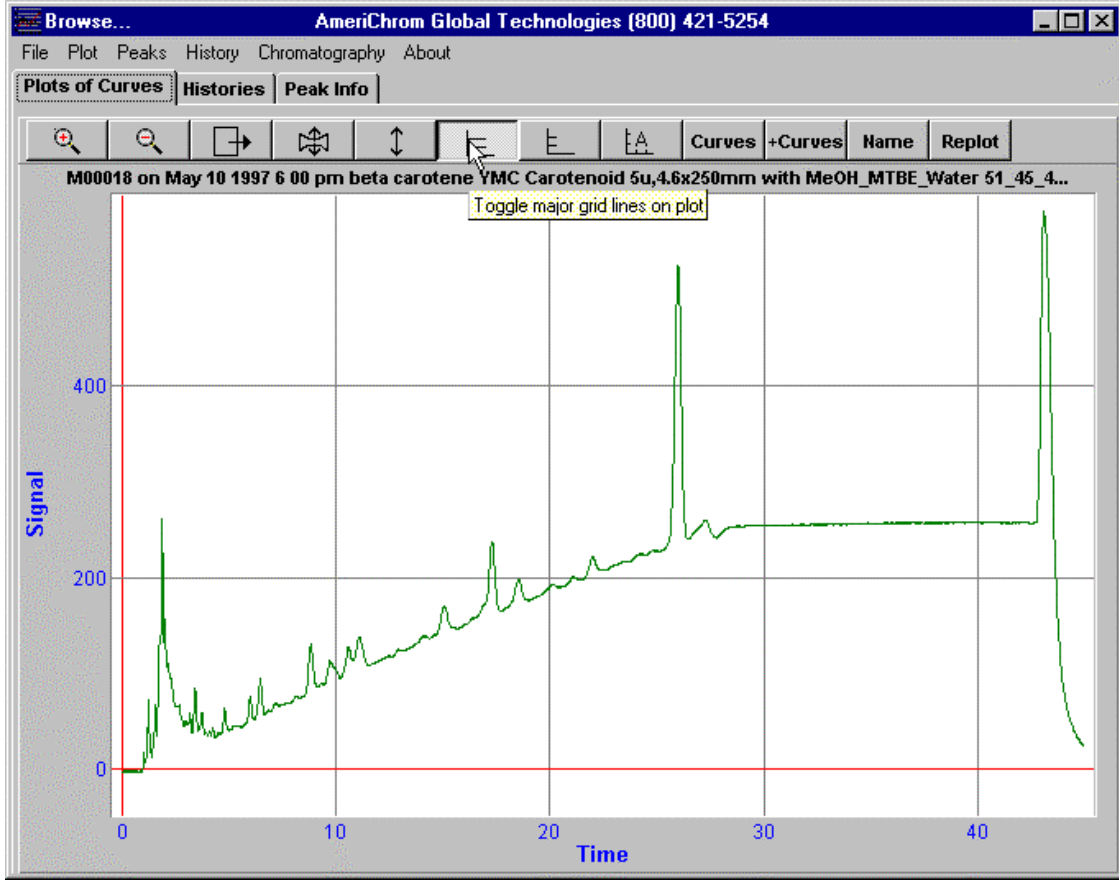




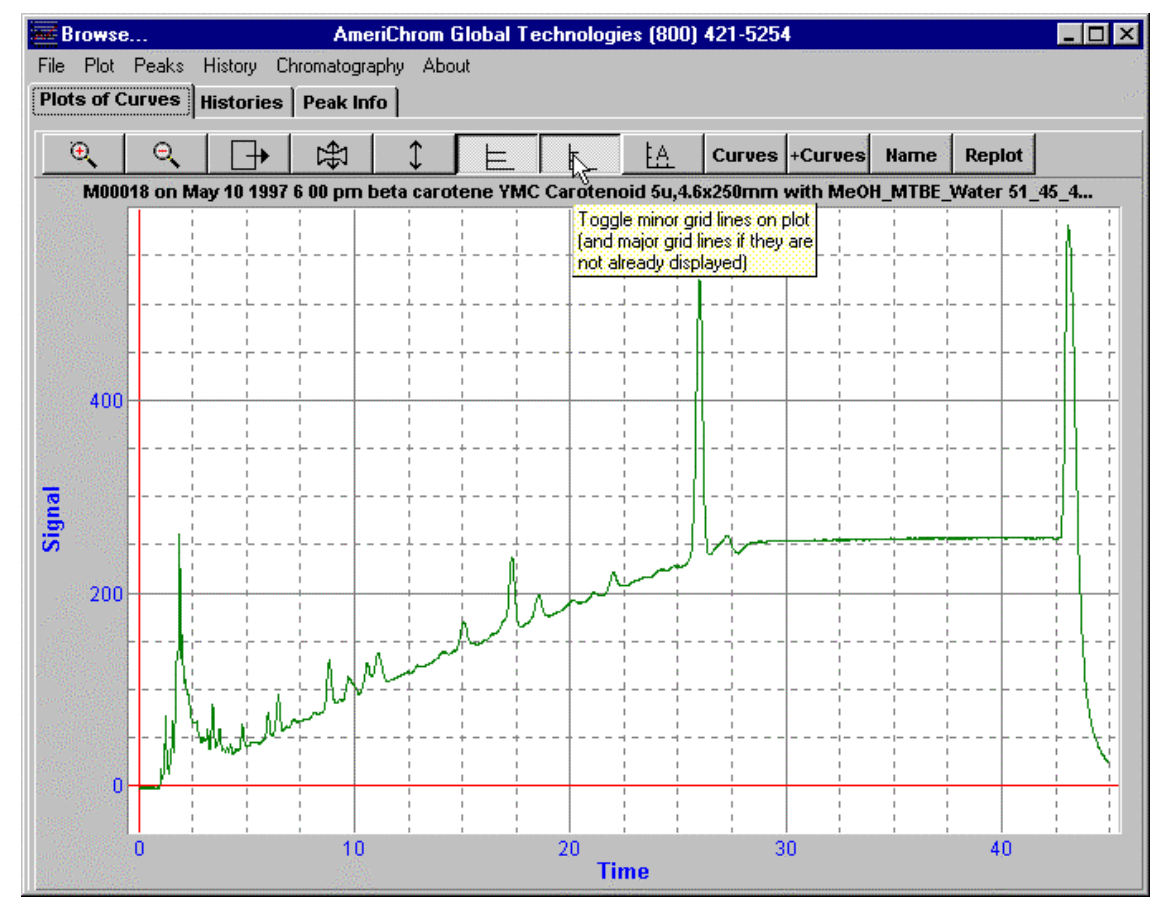

The plot title and visible limits may be changed by clicking on the eighth plot button, as shown below in the next four figures.

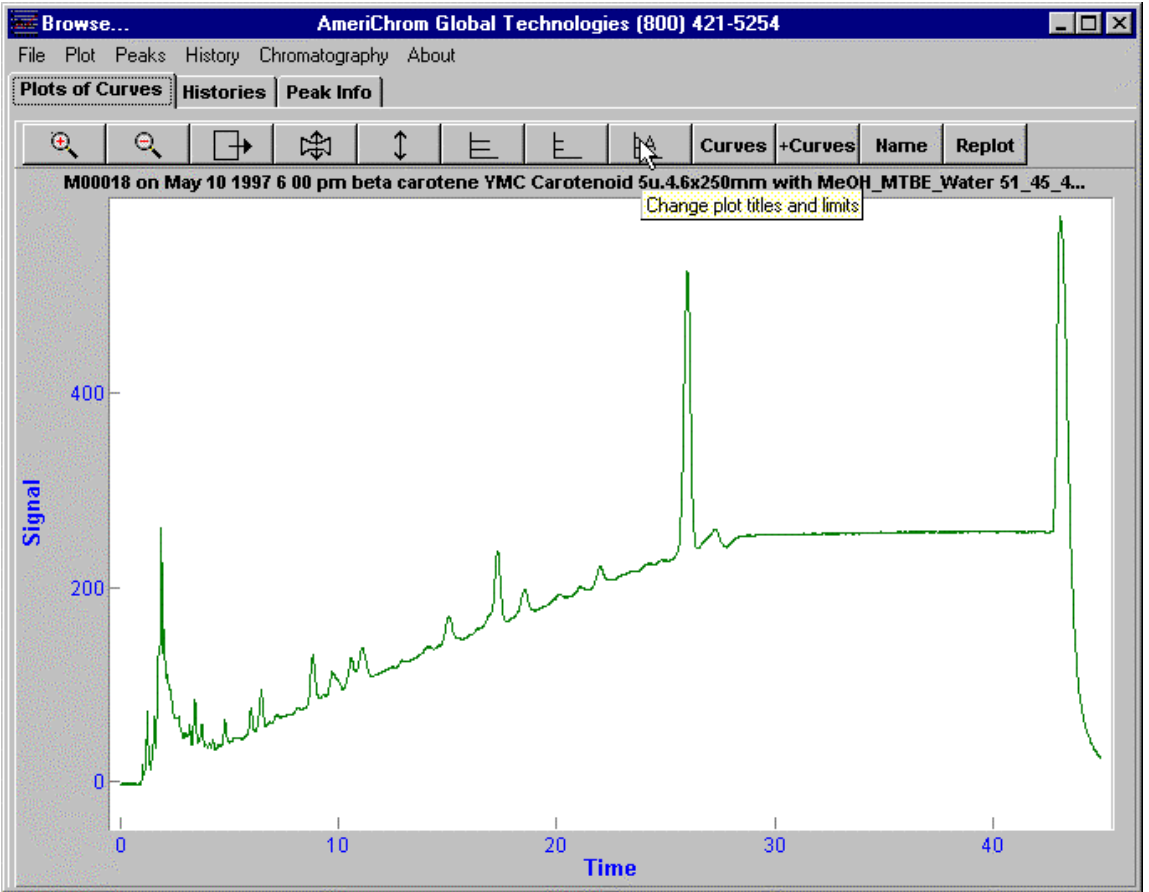


The original title was:

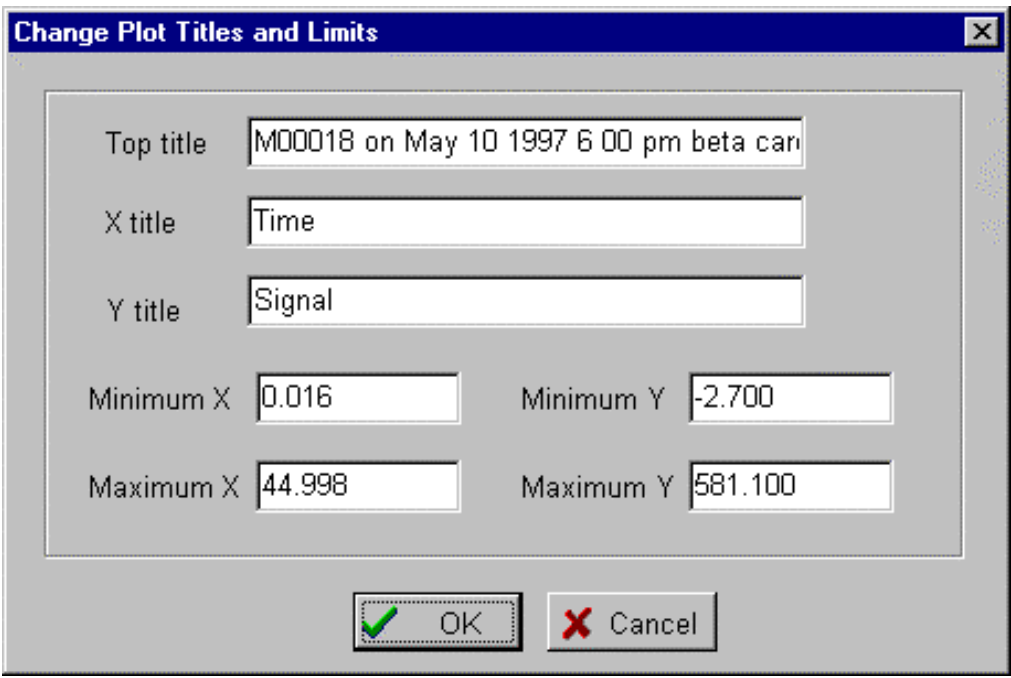

The new title will be:

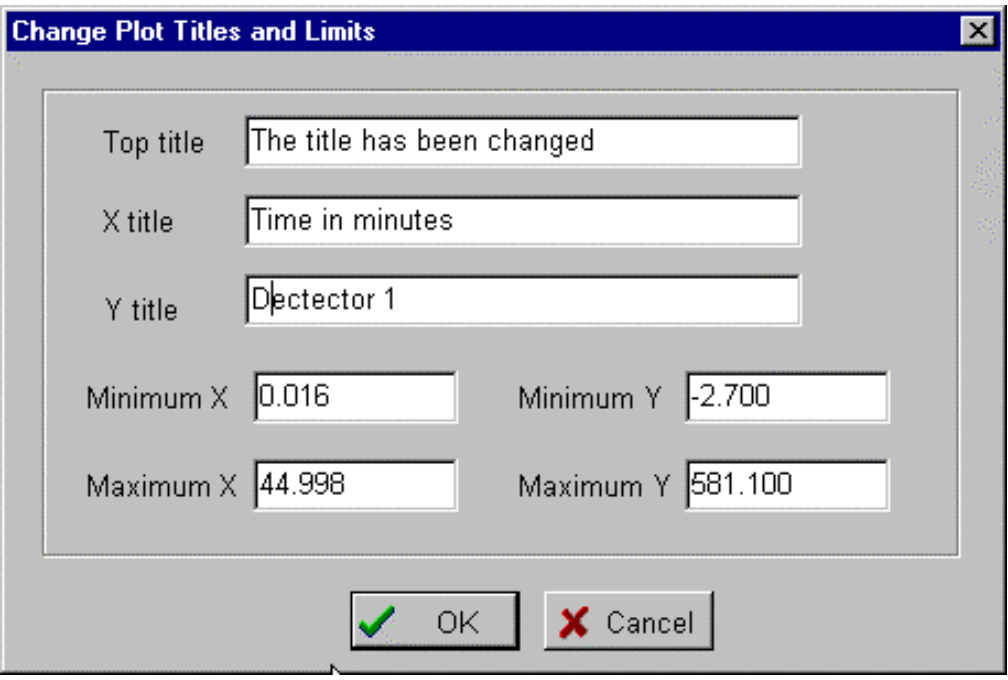

Clicking on the OK button in the dialog applies the changes. Clicking on the Cancel button abandons the changes. 


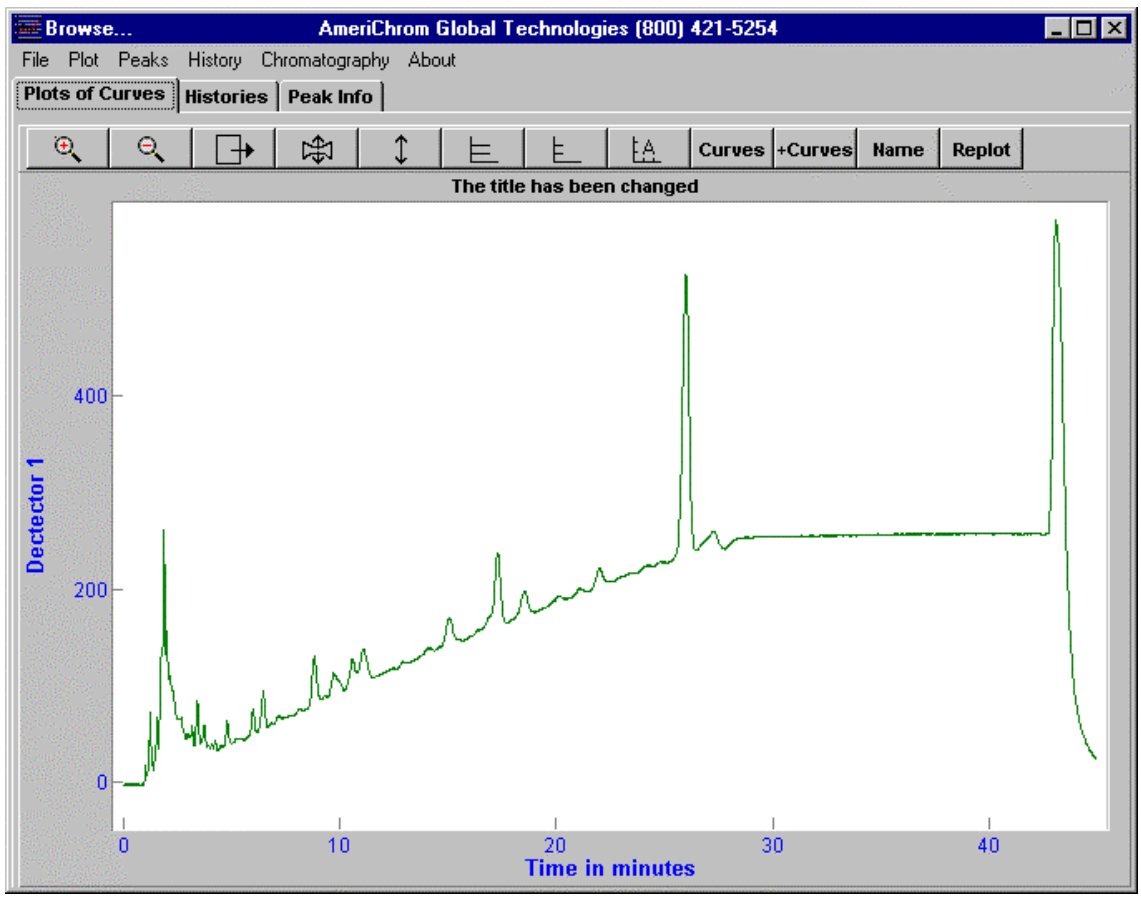

The uses of the remaining buttons will be shown after the example of correcting for baseline.

\section{Correcting for Baseline, an Example}

The ninth plot button brings up many features, as shown in the next two figures. 


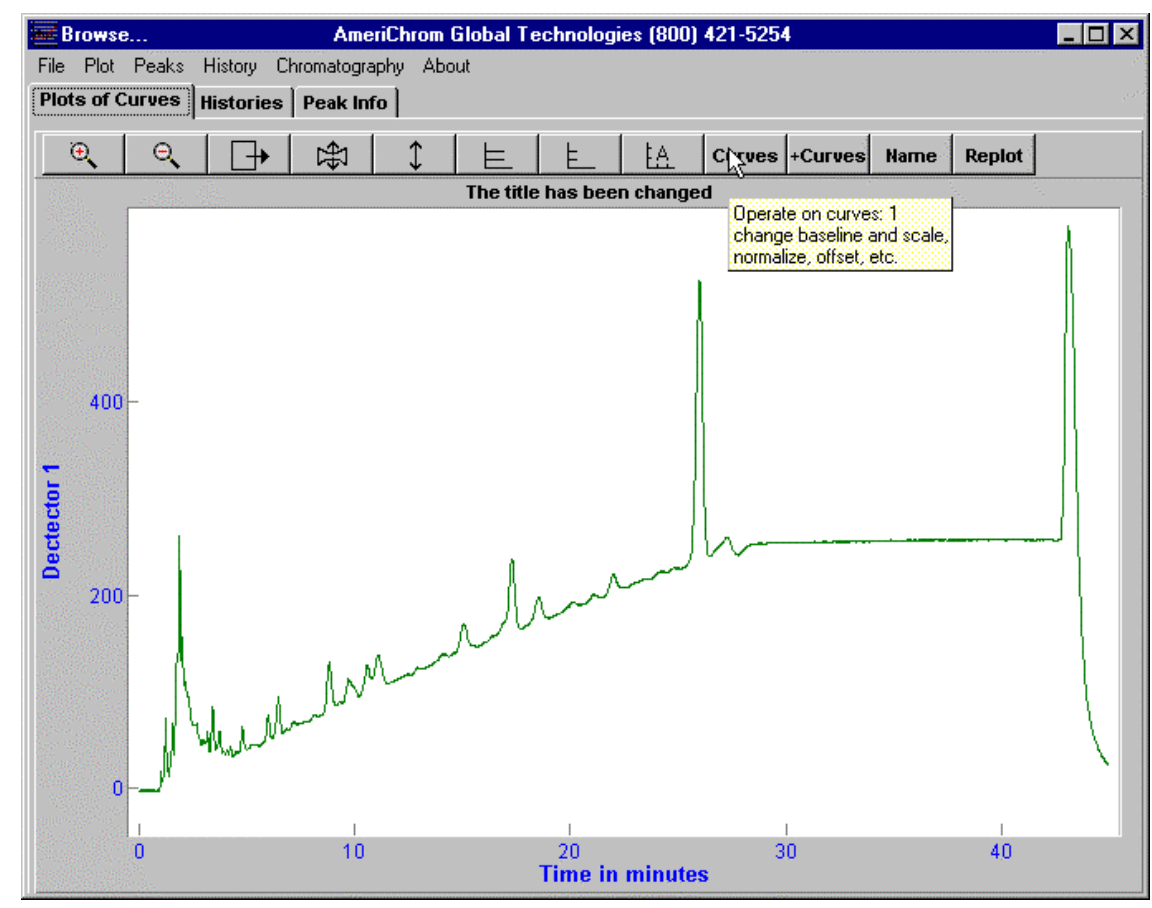

The default options in the dialog are shown in the next screen. Choices such as "None of the above" allow the operator to cancel a choice which would make a change to the plot.

\begin{tabular}{|c|c|}
\hline Operate on Curves & $\bar{x}$ \\
\hline $\begin{array}{l}\text { Change Baselines } \\
\mathrm{C} \text { Take zero from } \\
\mathrm{C} \text { Change lowest } \\
\text { c Zeros are OK }\end{array}$ & $\begin{array}{l}\text { efining Where Zero Is } \\
\text { cted point on curve } \\
\text { lue to zero }\end{array}$ \\
\hline Define Line Segme & \\
\hline $\begin{array}{l}\text { C Draw vertical lir } \\
\text { C Draw segments } \\
\text { C Remove vertica } \\
\text { C Remove line se } \\
\text { c None of the abo }\end{array}$ & $\begin{array}{l}\text { on plot } \\
\text { ween vertical lines } \\
\text { es } \\
\text { nts }\end{array}$ \\
\hline $\begin{array}{l}\text { Set Baselines after } \\
C \text { Baseline define } \\
C \text { Set all curve ba } \\
\varnothing \text { Baselines are } 0\end{array}$ & $\begin{array}{l}\text { wing Vertical Lines } \\
\text { tween vertical lines } \\
\text { nes to "Done" }\end{array}$ \\
\hline $\begin{array}{l}\text { Change Scales } \\
\bigcirc \text { Normalize curve } \\
0 \text { Scale curves } \\
0 \text { Scale curves by } \\
\odot \text { Scales are } 0 K\end{array}$ & $\begin{array}{l}\text { maximum height of } 1 \\
\text { clicked } \\
\text { ltiplication factor }\end{array}$ \\
\hline $\begin{array}{l}\text { Make Curves Visibl } \\
\text { All Curves Visib } \\
\text { C Choose Visible } \\
6 \text { Leave As Is }\end{array}$ & $\begin{array}{l}\text { Invisible } \\
\text { es }\end{array}$ \\
\hline Г OffSet Curves & \\
\hline$\checkmark \quad 0 K$ & X Cancel \\
\hline
\end{tabular}


For example, if one wanted to remove the sloping baseline from the displayed plot, the way to do that would be to define some vertical lines, the intersection of which with the curve would define the new zeros on the curve, draw in some connecting lines along the curve to see how things looked, then judge by eye when the process was satisfactory and apply the changes. A typical baseline removal sequence will now be demonstrated.

First, we tell the plot we are going to draw vertical lines by selecting that choice, as seen in the dialog where the cursor arrow is.

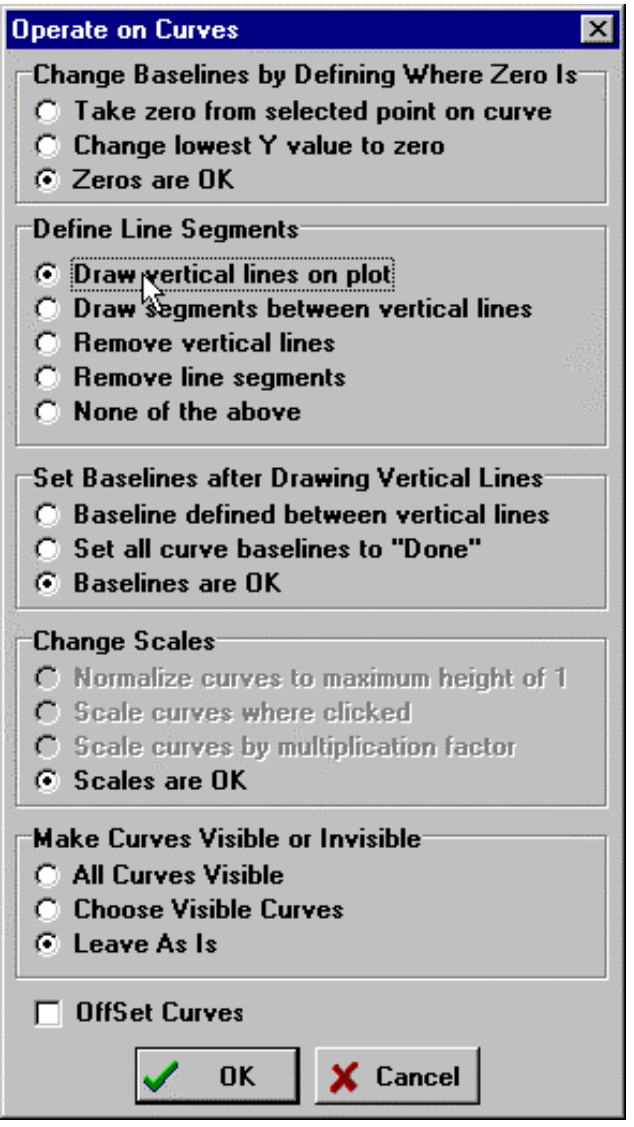

After we have clicked on OK to activate the process, each right click with the mouse draws a vertical line where the mouse cursor is, as shown in the following figure. 


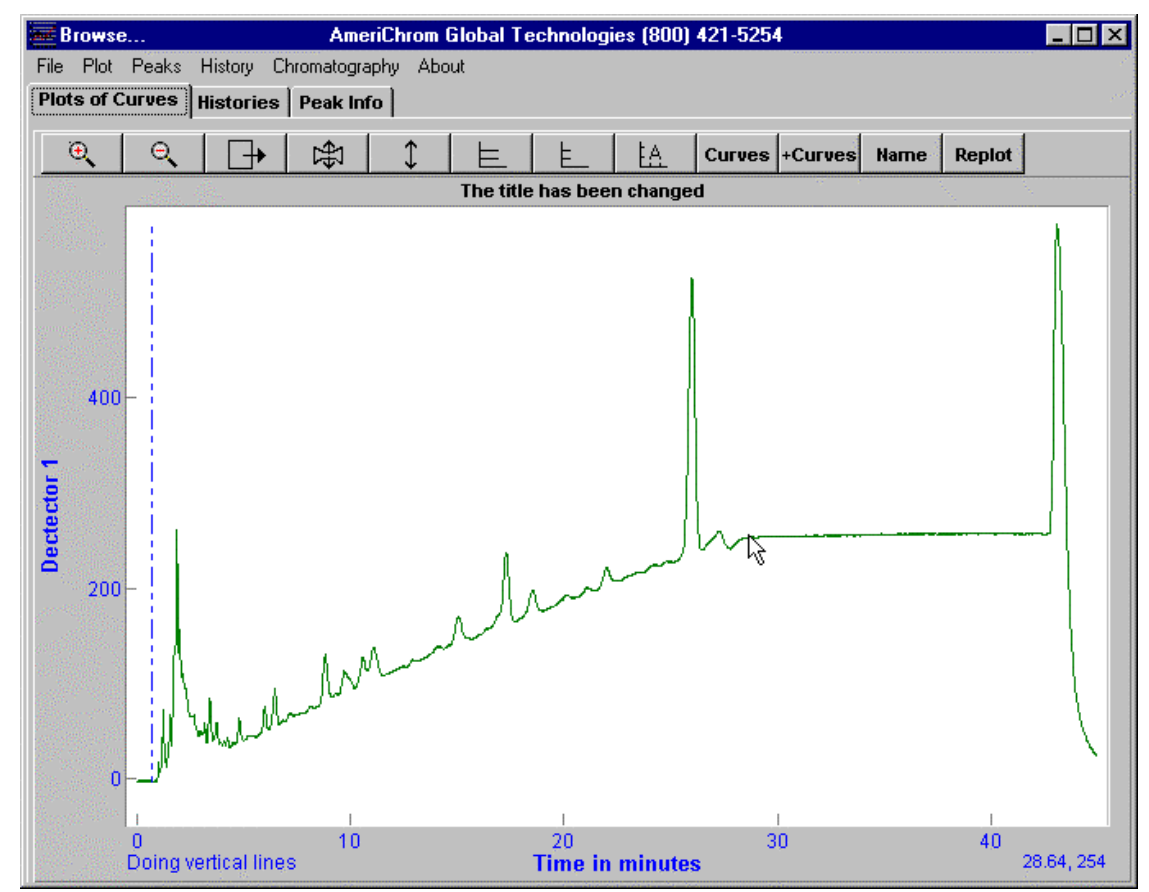

After some lines have been drawn (presumably at least two), segments between vertical lines should be drawn to see how well the segments follow the curve and hence to see if enough vertical lines have been drawn to allow a reasonable subtraction of the slope in the curve. Select the "Draw segments between vertical lines" option in the dialog box.

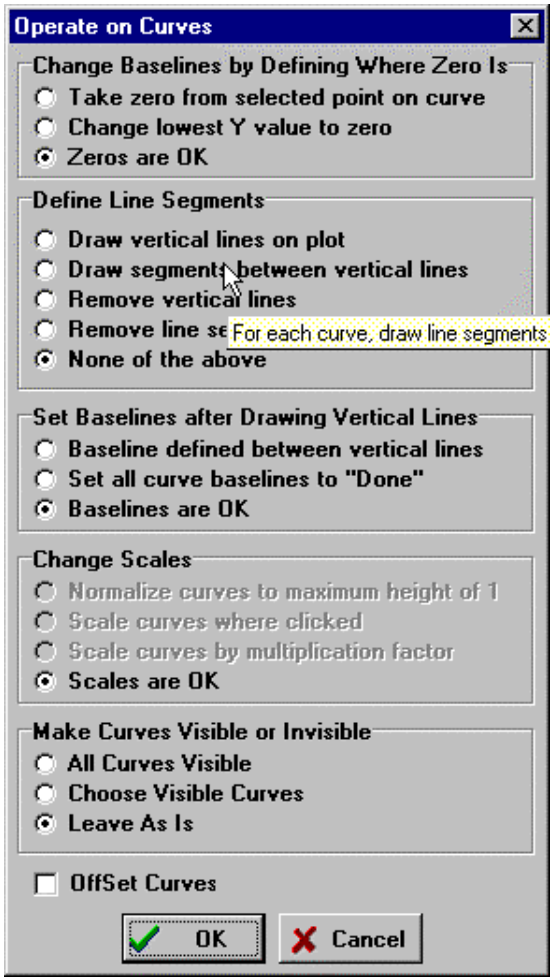


Clicking on OK activates the process, clicking on Cancel abandons it. Since we clicked on OK, we get the plot shown below. The segments do not match the curve well enough between say 10 and 30 minutes, and more vertical lines will have to be drawn. Up to 100 vertical lines can be active simultaneously.

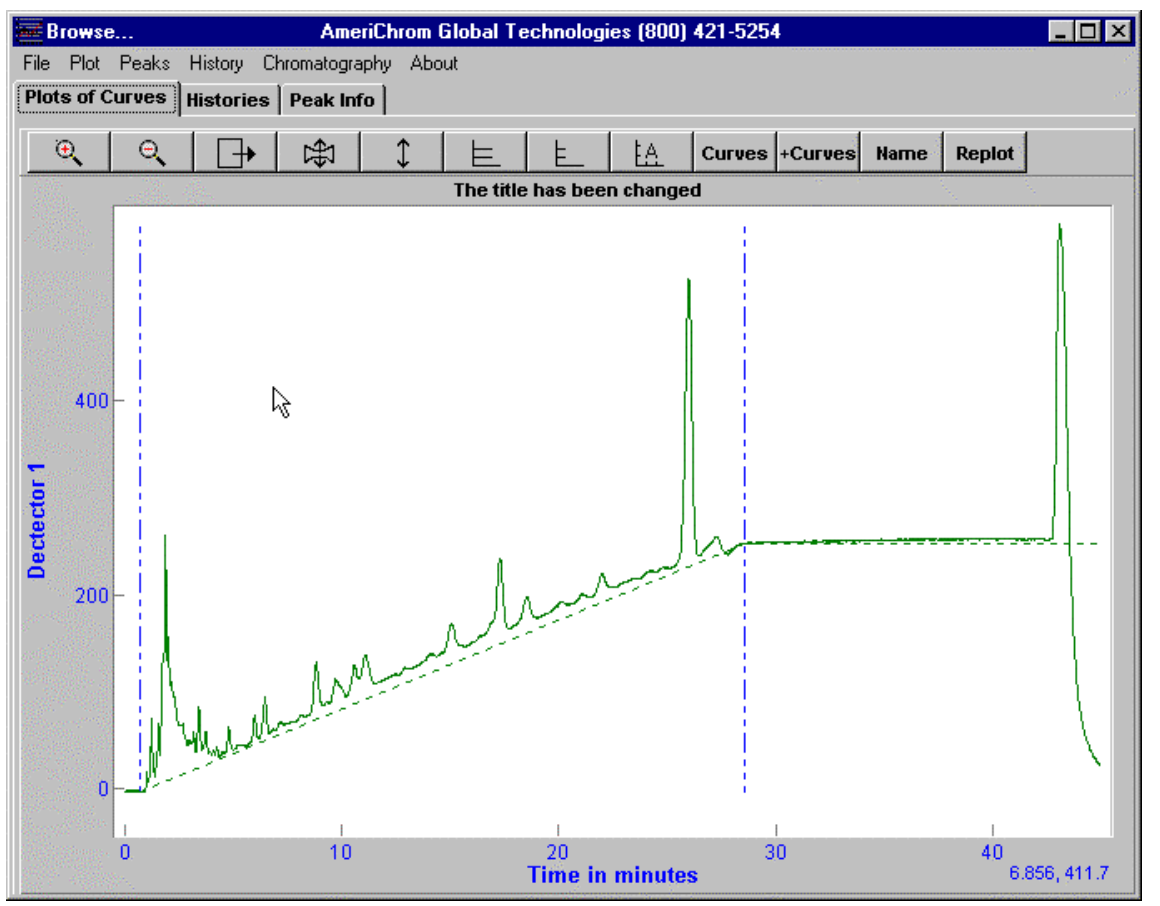

We bring up the dialog again by clicking on the ninth plot button and select "Draw vertical lines on plot". 


\begin{tabular}{|c|c|}
\hline \multicolumn{2}{|l|}{ Operate on Curves } \\
\hline \multicolumn{2}{|c|}{$\begin{array}{l}\text { Change Baselines by Defining Where Zero Is } \\
\mathrm{C} \text { Take zero from selected point on curve } \\
\mathrm{C} \text { Change lowest } \mathrm{Y} \text { value to zero } \\
\text { C Zeros are } \mathrm{OK}\end{array}$} \\
\hline \multicolumn{2}{|c|}{ Define Line Segments } \\
\hline \multicolumn{2}{|c|}{$\begin{array}{l}\odot \text { Praw vertical lines on plot } \\
C \text { Draw segments between vertical lines }\end{array}$} \\
\hline \multicolumn{2}{|c|}{$\begin{array}{l}\text { C While this is selected, clicks on plot draw vertical line } \\
\text { hemove ilne segiments }\end{array}$} \\
\hline \multicolumn{2}{|c|}{$\begin{array}{l}\text { Set Baselines after Drawing Vertical Lines- } \\
\bigcirc \text { Baseline defined between vertical lines } \\
\text { S Set all curve baselines to "Done" } \\
\sigma \text { Baselines are } 0 \mathrm{~K}\end{array}$} \\
\hline \multicolumn{2}{|c|}{$\begin{array}{l}0 \text { Normalize curves to maximum height of } 1 \\
0 \text { Scale curves where clicked } \\
\text { Scale curves by multiplication factor } \\
\text { c Scales are } \mathbf{0 K}\end{array}$} \\
\hline \multicolumn{2}{|c|}{$\begin{array}{l}\text { Make Curves Visible or Invisible } \\
\mathrm{C} \text { All Curves Visible } \\
\mathrm{C} \text { Choose Visible Curves } \\
\odot \text { Leave As Is }\end{array}$} \\
\hline \multicolumn{2}{|l|}{ Г OffSet Curves } \\
\hline \begin{tabular}{|ll}
$\quad \mathrm{OK}$ \\
\end{tabular} & X Cancel \\
\hline
\end{tabular}

After clicking on OK, we get the plot back and draw more vertical lines where we want to force the segments to match the curve more closely. 


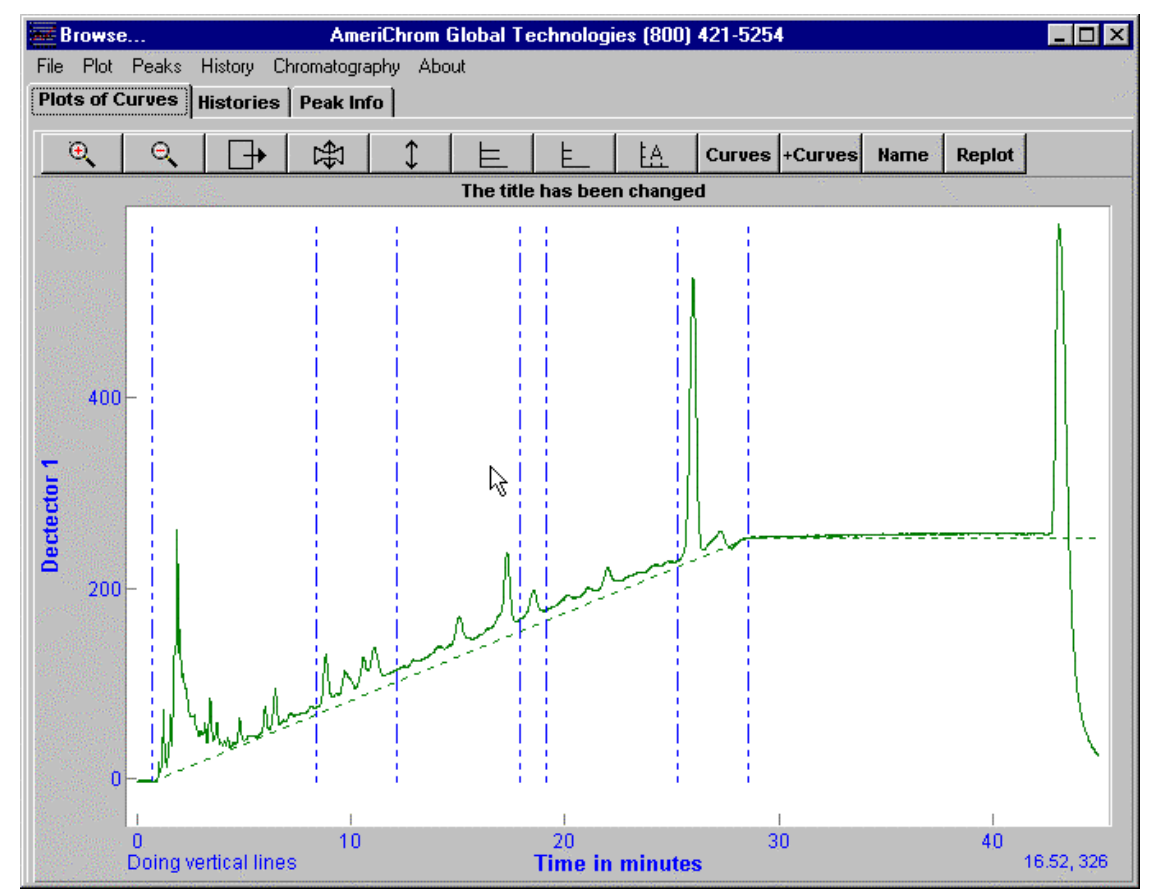

We have not redrawn the segments yet, so they still do not look satisfactory. We bring up the dialog, select "Draw segments between vertical lines", click on OK, and obtain the following result. This is much better between 10 and 30 minutes that the previous attempt, but is still not "right" beyond 30 minutes.

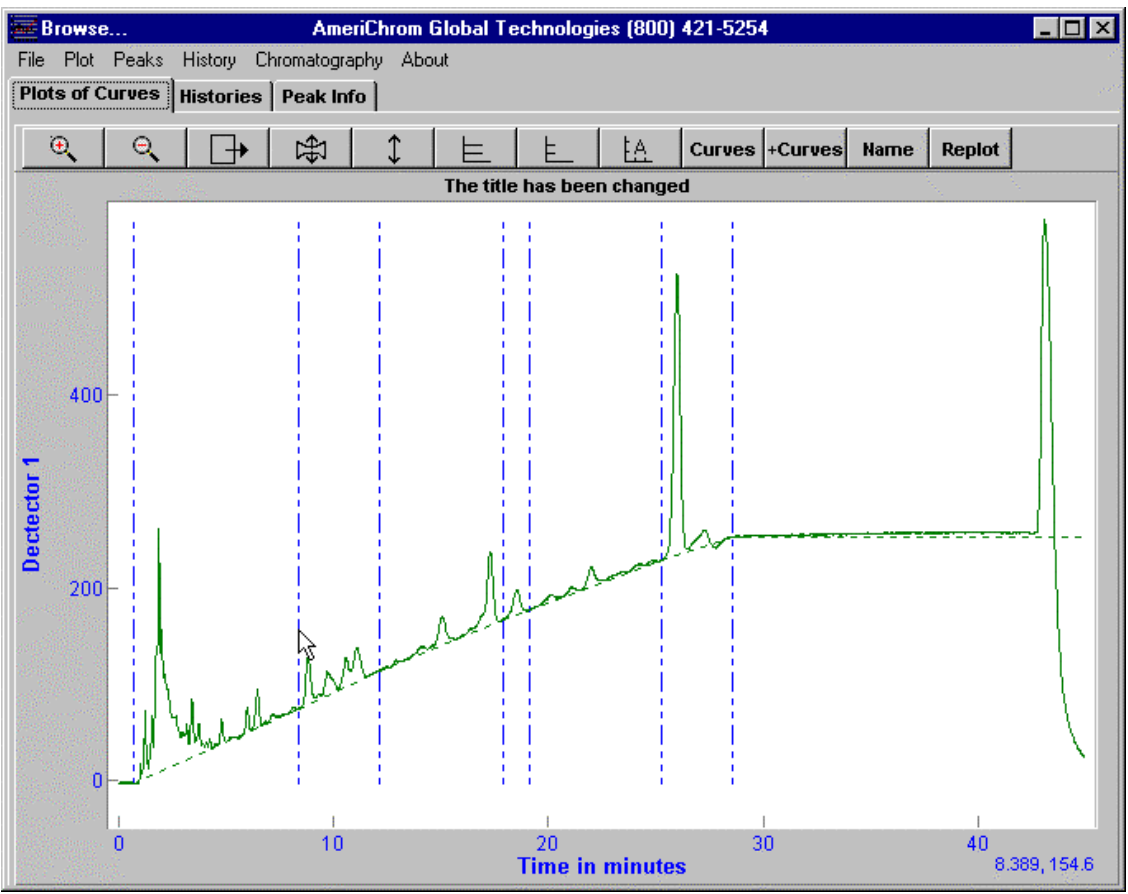


The solution is to draw another vertical line near 40 minutes and draw the segments again to see how things now look. The result is shown below.

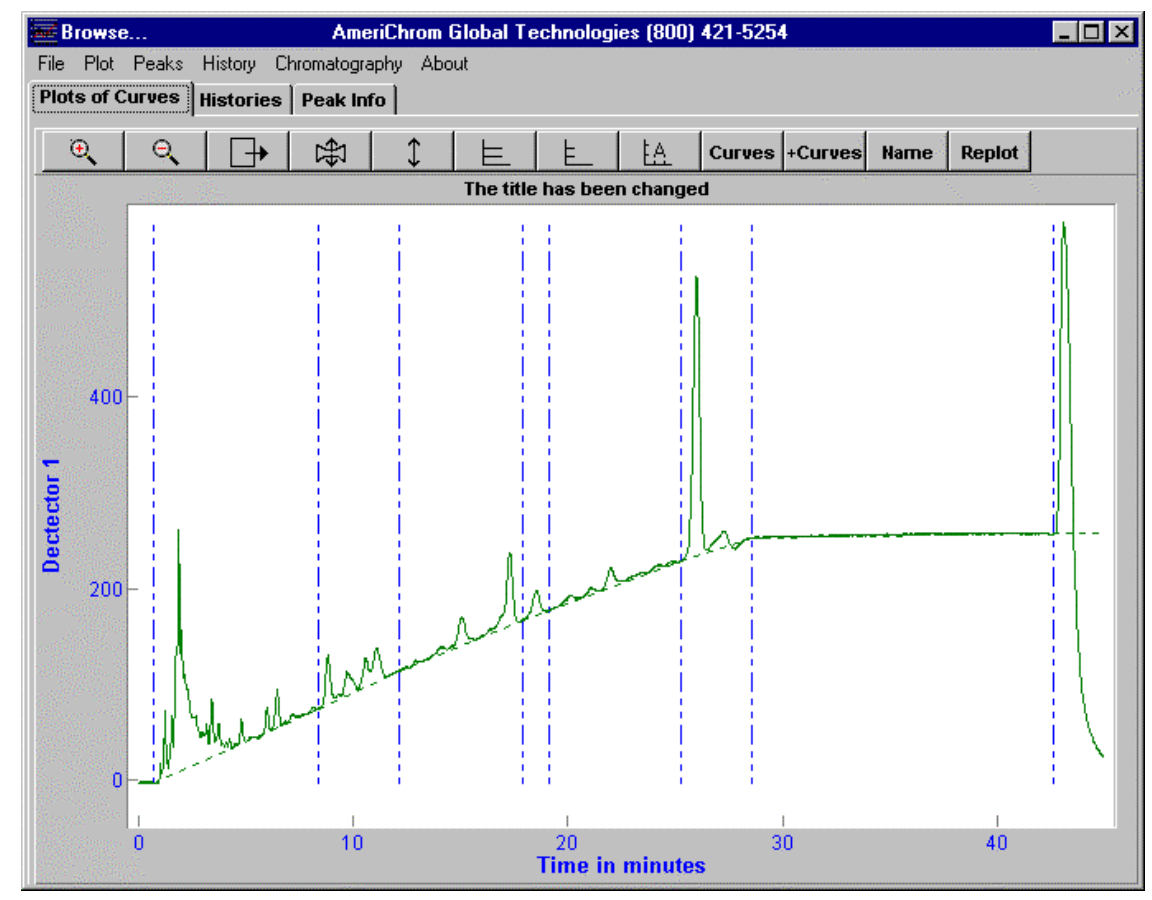

This is now satisfactory and we can subtract the line segments from the original curve to remove the effect of the slope. We bring up the dialog, select "Baseline defined between vertical lines", click on OK, and have the result: 


\begin{tabular}{|c|c|}
\hline \multicolumn{2}{|l|}{ Operate on Curves } \\
\hline \multicolumn{2}{|c|}{$\begin{array}{l}\text { Change Baselines by Defining Where Zero Is } \\
\mathrm{C} \text { Take zero from selected point on curve } \\
\mathrm{C} \text { Change lowest } Y \text { value to zero } \\
\text { C Zeros are } 0 \mathrm{~K}\end{array}$} \\
\hline \multicolumn{2}{|c|}{ Define Line Segments } \\
\hline \multicolumn{2}{|c|}{$\begin{array}{l}C \text { Draw vertical lines on plot } \\
C \text { Draw segments between vertical lines } \\
\mathrm{C} \text { Remove vertical lines } \\
\mathrm{O} \text { Remove line segments } \\
6 \text { None of the above }\end{array}$} \\
\hline \multicolumn{2}{|c|}{ Set Baselines after Drawing Vertical Lines } \\
\hline \multicolumn{2}{|c|}{$\begin{array}{l}\text { Baseline defined between vertical lines } \\
\text { Set all curve baselines to "Done" }\end{array}$} \\
\hline \multicolumn{2}{|c|}{ Correct for a sloping baseline by forcing the baseline thro } \\
\hline \multicolumn{2}{|c|}{$\begin{array}{l}\text { Change Scales } \\
0 \text { Normalize curves to maximum height of } 1 \\
0 \text { Scale curves where clicked } \\
0 \text { Scale curves by multiplication factor } \\
6 \text { Scales are } \mathbf{0 K}\end{array}$} \\
\hline \multicolumn{2}{|c|}{$\begin{array}{l}\text { Make Curves Visible or Invisible- } \\
\text { C All Curves Visible } \\
\text { C Choose Visible Curves } \\
\text { (c Leave As Is }\end{array}$} \\
\hline \multicolumn{2}{|l|}{ Г OffSet Curves } \\
\hline$\checkmark \quad$ OK & X Cancel \\
\hline
\end{tabular}

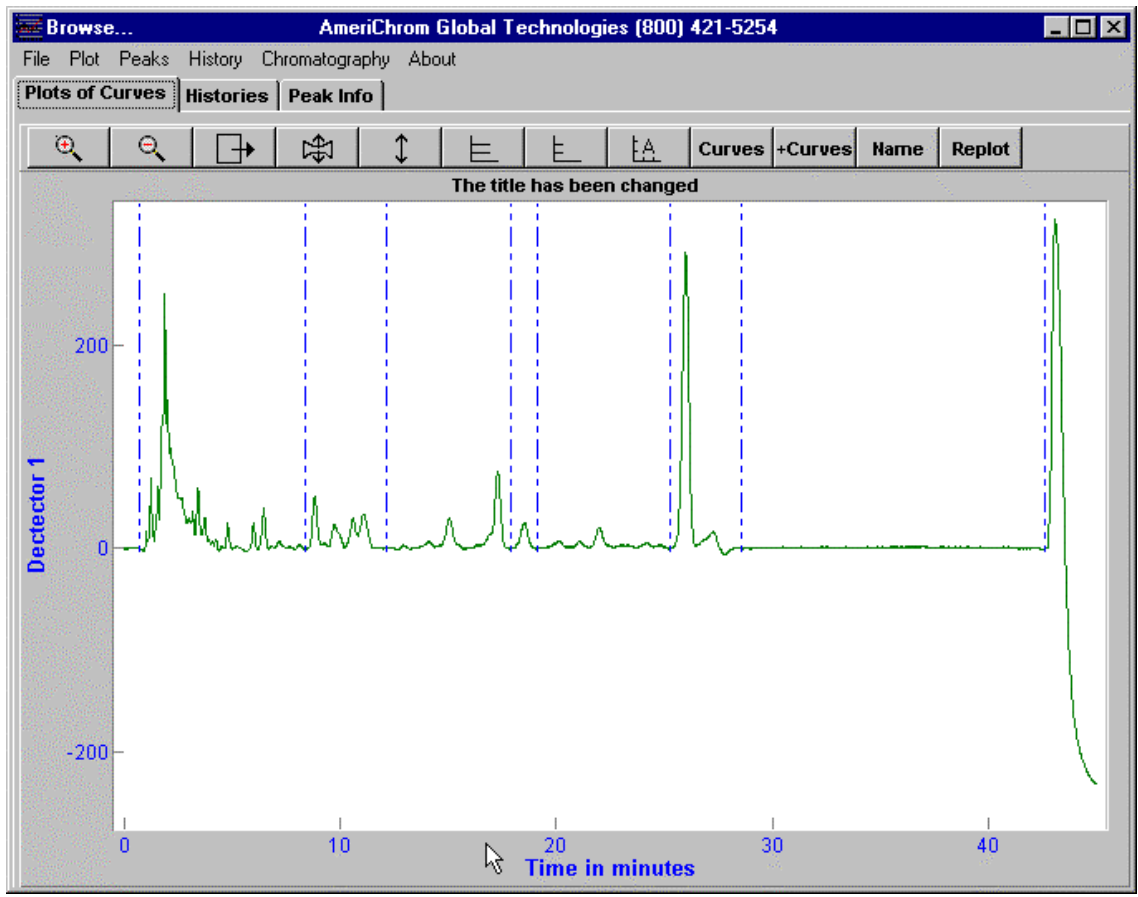


This is just what we need. The vertical lines are now superfluous and can be removed from the dialog, viz.:

\begin{tabular}{|c|c|}
\hline \multicolumn{2}{|l|}{ Operate on Curves } \\
\hline \multicolumn{2}{|c|}{$\begin{array}{l}\text { Change Baselines by Defining Where Zero Is } \\
\mathrm{O} \text { Take zero from selected point on curve } \\
\mathrm{C} \text { Change lowest } \mathrm{Y} \text { value to zero } \\
6 \text { Zeros are } \mathrm{OK}\end{array}$} \\
\hline \multicolumn{2}{|c|}{ Define Line Segments } \\
\hline \multicolumn{2}{|c|}{$\begin{array}{l}\text { Draw vertical lines on plot } \\
\text { Draw segments between vertical lines } \\
\text { Remove vertical lines } \\
\text { Remove line segments }\end{array}$} \\
\hline Removes any user-d & n vertical lines. \\
\hline \multicolumn{2}{|c|}{$\begin{array}{l}\text { Set Baselines after Drawing Vertical Lines } \\
\text { Baseline defined between vertical lines } \\
\text { Set all curve baselines to "Done" } \\
\text { C Baselines are 0K }\end{array}$} \\
\hline \multicolumn{2}{|c|}{$\begin{array}{l}\text { Change Scales } \\
C \text { Normalize curves to maximum height of } 1 \\
\text { Scale curves where clicked } \\
\text { S Scale curves by multiplication factor } \\
\text { C Scales are } 0 \mathrm{~K}\end{array}$} \\
\hline \multicolumn{2}{|c|}{$\begin{array}{l}\text { Make Curves Visible or Invisible } \\
\text { All Curves Visible } \\
\text { Choose Visible Curves } \\
6 \text { Leave As Is }\end{array}$} \\
\hline \multicolumn{2}{|l|}{ Г OffSet Curves } \\
\hline \begin{tabular}{|l|} 
\\
\end{tabular} & X Cancel \\
\hline
\end{tabular}

The result is: 


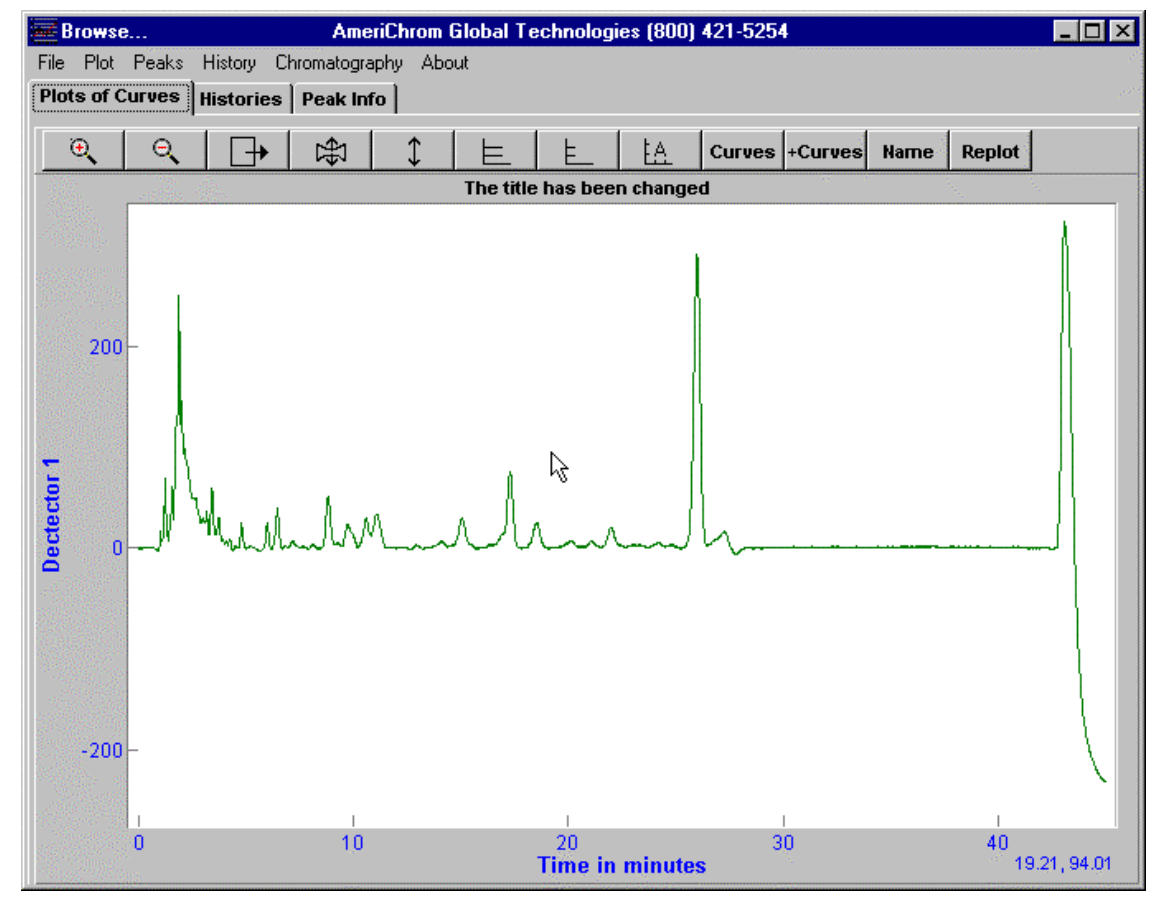

\section{Curve Arithmetic}

Four more plot buttons (a Clear button, not shown in the figure, has recently been added) remain to be explained. The tenth button also has several uses, but only one will be mentioned here - cutting "useless" data off a curve. Other uses will be shown under "working with several curves".

In the plot of the chromatogram where we removed the slope from the baseline, the peak above 40 minutes is meaningless and should be removed. This can easily be done by first zooming the plot so that the desired part of the data is still visible, then clicking on the tenth plot button to bring up a dialog which allows the curve to be cut so that it only contains the data in the visible part. The process is demonstrated below.

First, we zoom the plot to so only the desired part is visible. 


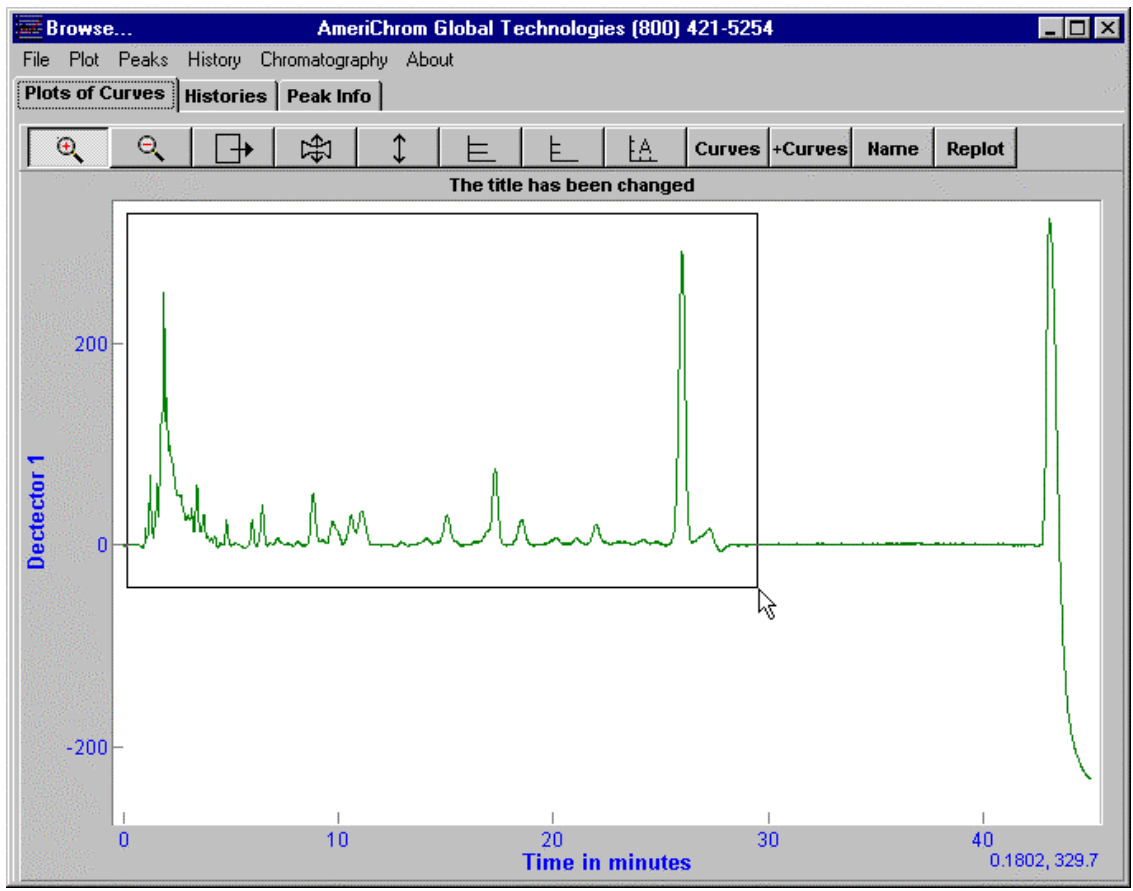

The result is:

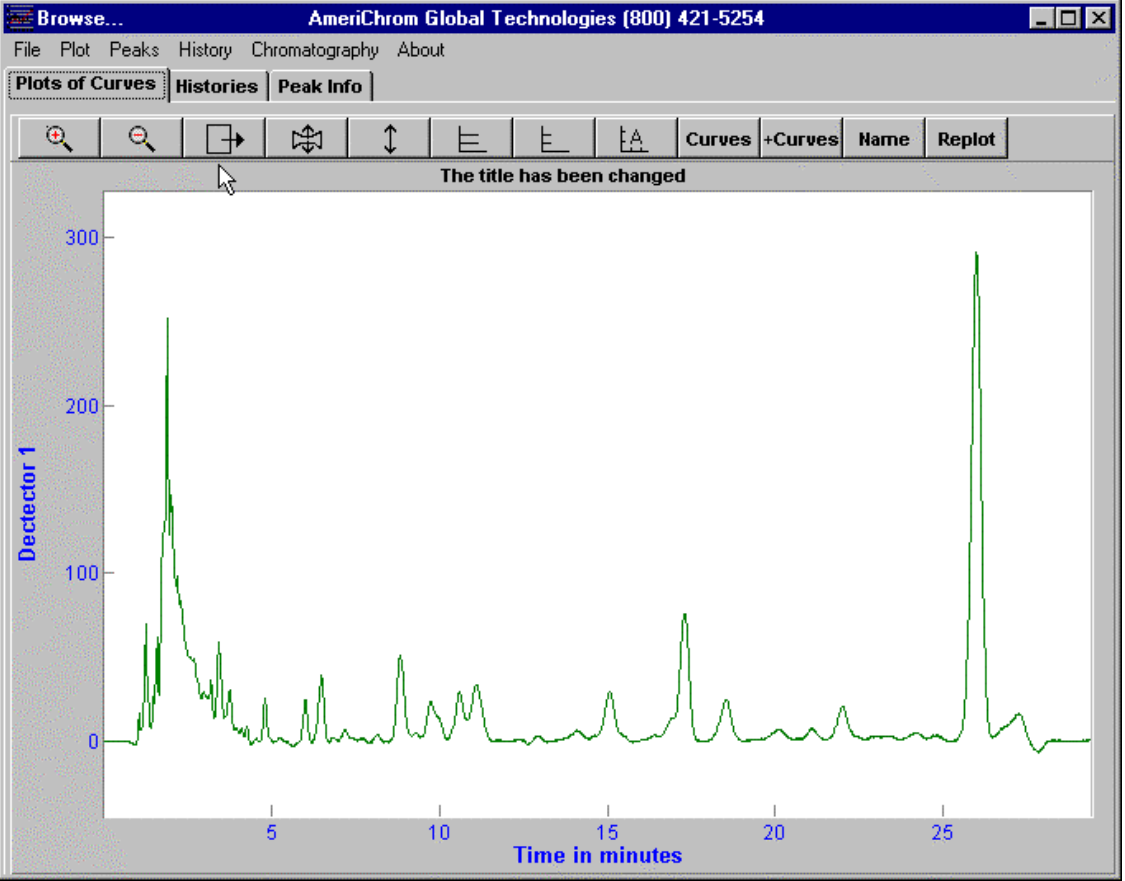

We click on the tenth plot button: 


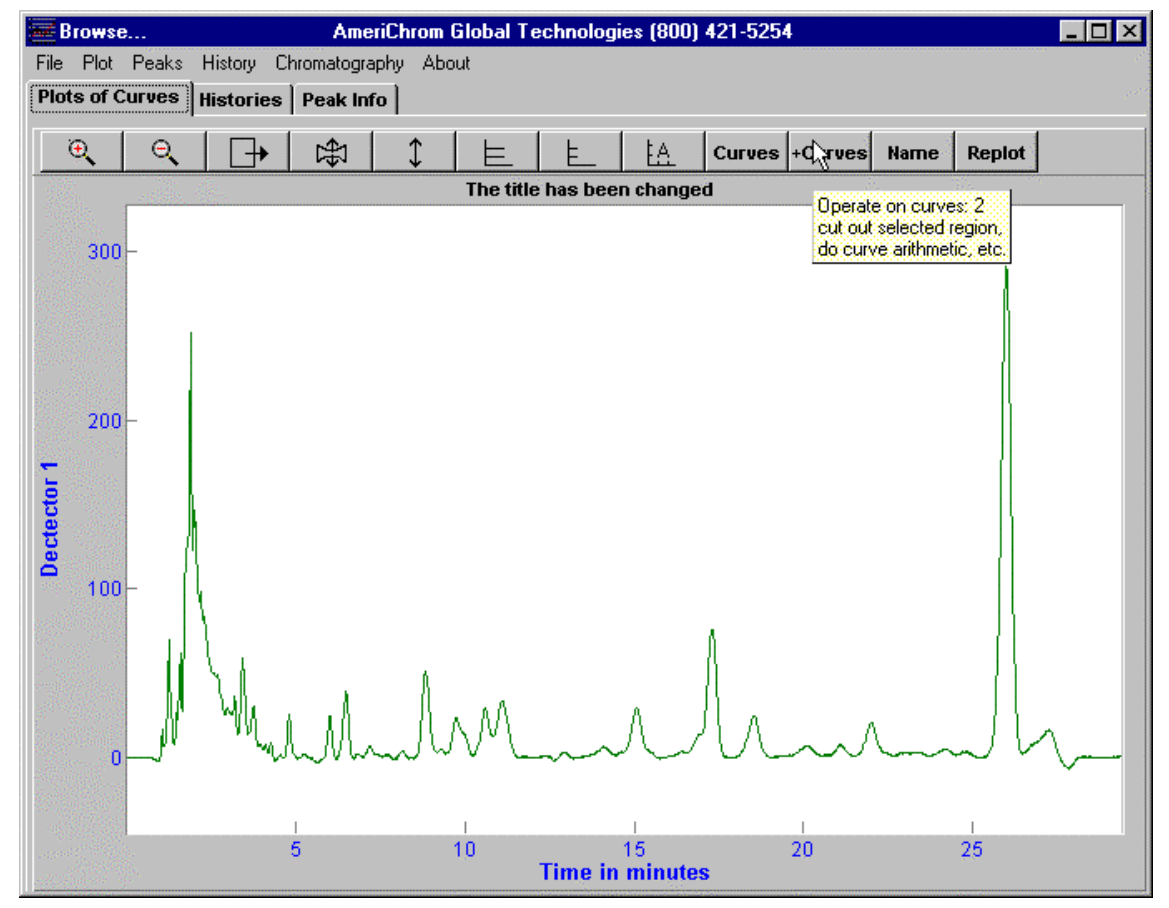

We change the radio button from "Do nothing" to "Cut curves as shown" and exit by clicking on OK.

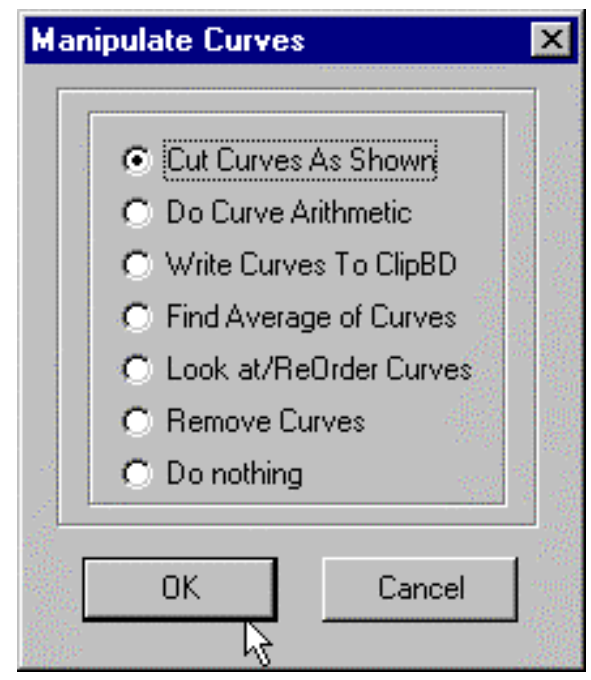

We then have only the visible data in the plot. Because we have expended some effort to get to this stage, we will now save the data in ASCIl format so that if need be (for example one of the figures in this manuscript gets lost) we can pick up from where we were. Normally, 
one would not need to save the data in ASCII but here we want to transfer the curve data to a program to produce a publication-quality figure.

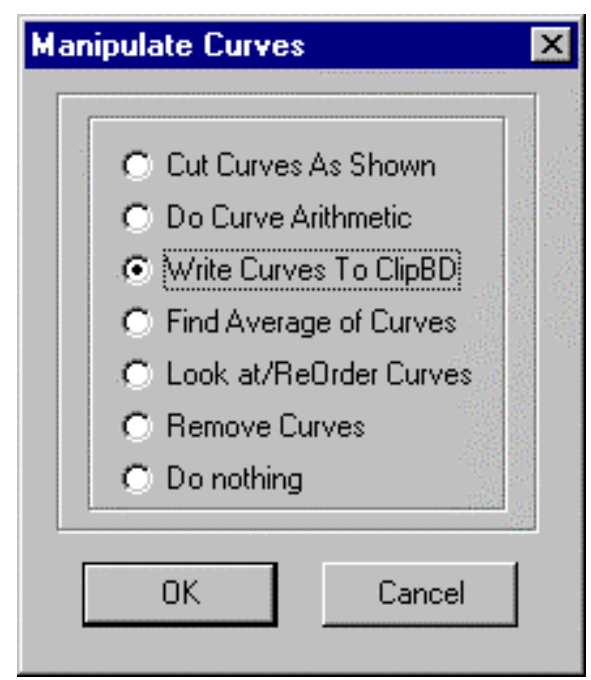

We click on OK. The curve data are written to the clipboard. The clipboard now contains:

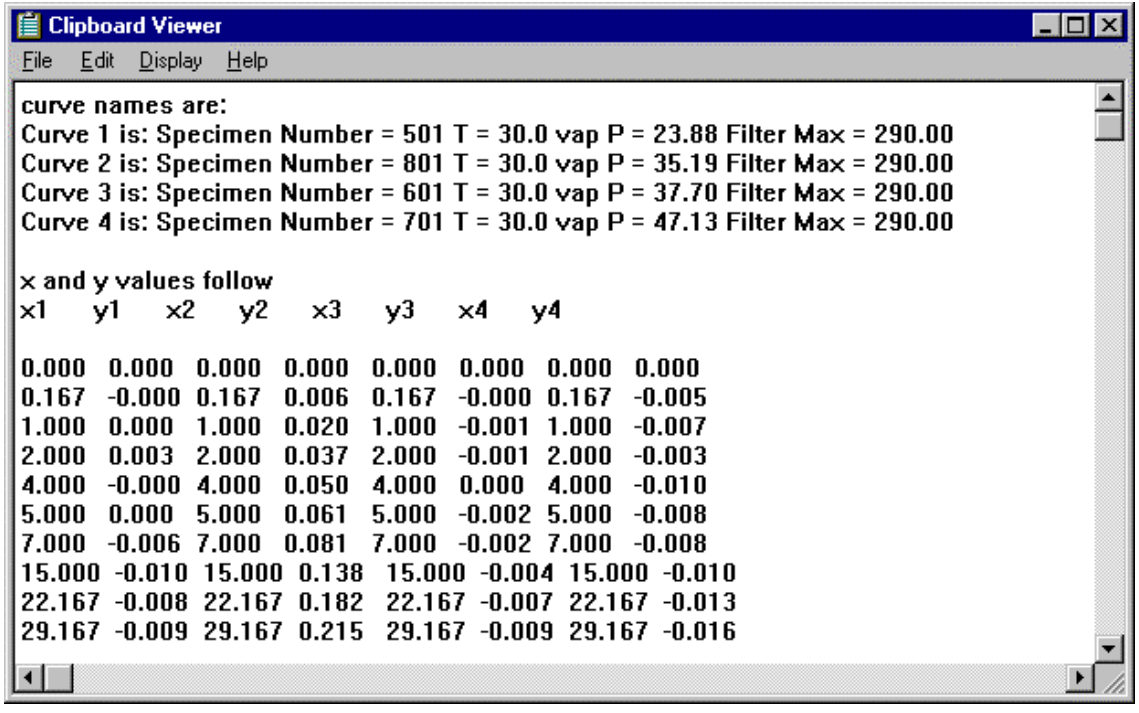

\section{Working with Several Curves}

The options brought up by clicking on the curves+ button over the plots will be discussed more in the sections on Results Processing Programs. 


\section{Results Comparison Programs}

The programs which allow the user to examine and compare the results of assessing the UV dosage and damage undergone by a specimen are still being developed. At the moment, these are

1) Look at IR

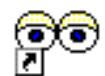

Shortcut to LookAtIRA.e..

which allows the user to look at the damage, i.e., the changes in the IR spectra at some particular wavelength chosen in the data processing program, and

2) Look at Damage

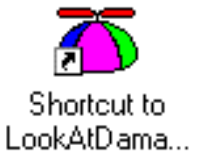

which plots initial quantum yield as a function of filter wavelength, or temperature, or relative humidity.

These two programs allow the experimental evidence to be examined and verified and the results to be shown in an understandable way so that they can be monitored for self-consistency.

\section{LookAtIR program}

The LookAtIR program is provided to allow the user to examine the relationships between damage and time and dosage and time without the complicating aspect of relating damage to dosage. Some provision has been made to examine damage as a function of $\mathrm{RH}$. The capability of examining these intermediate states of the ultimately desired analysis of damage as a function of dosage significantly eases 1) understanding how the value of a given result was derived and 2) verifying whether individual results are part of an overall pattern or are suspiciously outlying. 
When the LookAtIR begins, it requires a damage database table from which to read the experimental conditions. A standard Windows file open box appears:

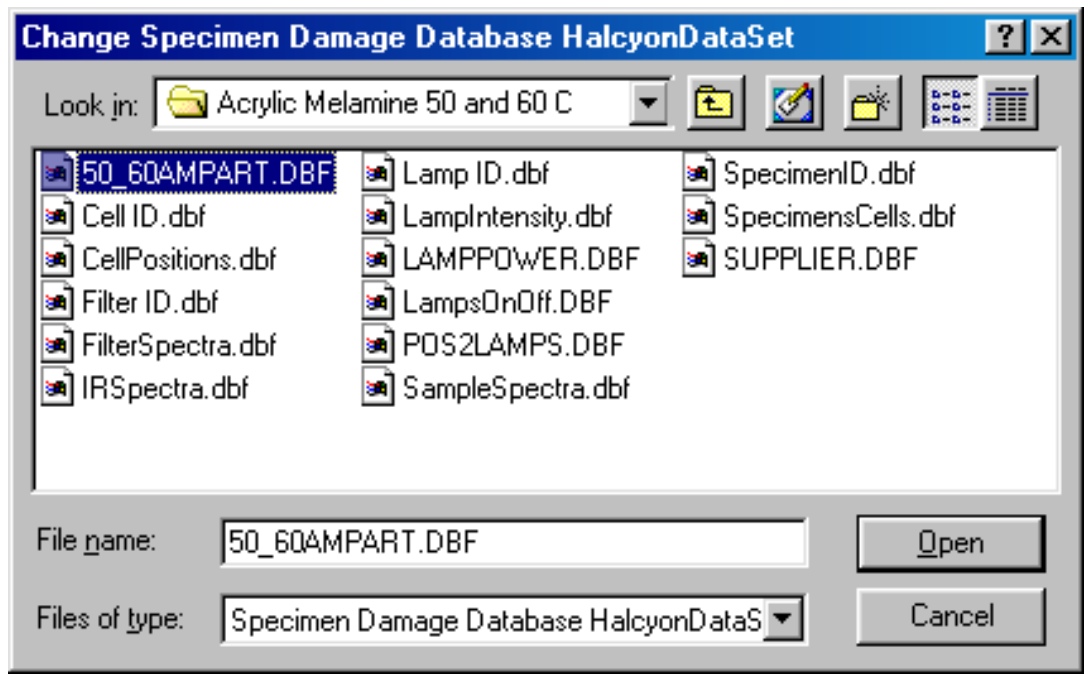

After the database table has been linked to the program, the user can begin to choose the information he wishes to see. The first page of the program is concerned with damage as a function of time in the top plot and damage as a function of $\mathrm{RH}$ or temperature in the bottom plot. The bottom plot is generated from information put into the top plot.

Choices are executed using the buttons on the right hand side of the plot window. The damage site is selected from those available in the database table as shown in a drop down list box. The drop down list removes the necessity that the user remember which damage sites were used. This information is extracted from the database table by the program as the database table is read in.

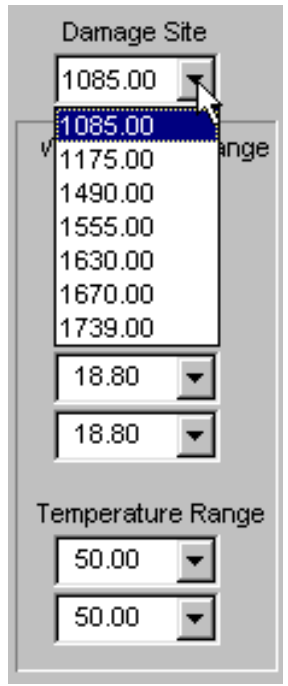


The UV wavelength range for which to see the damage must also be chosen from its drop down list box.

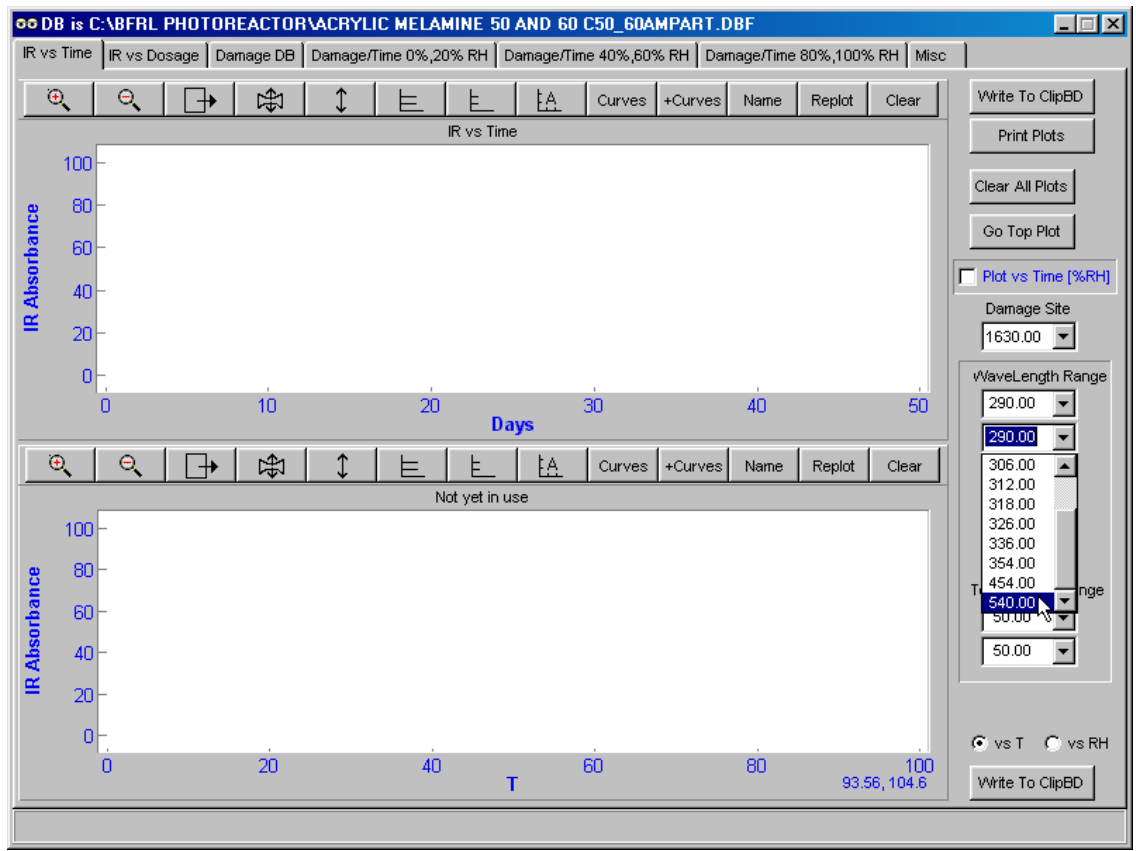

If plots are desired of damage versus temperature interpolated to various levels of humidity (currently fixed at $0 \%, 20 \%, 40 \%, 60 \%, 80 \%$ and $100 \%$ ), the box "Plot vs Time [RH\%]"should be checked. 


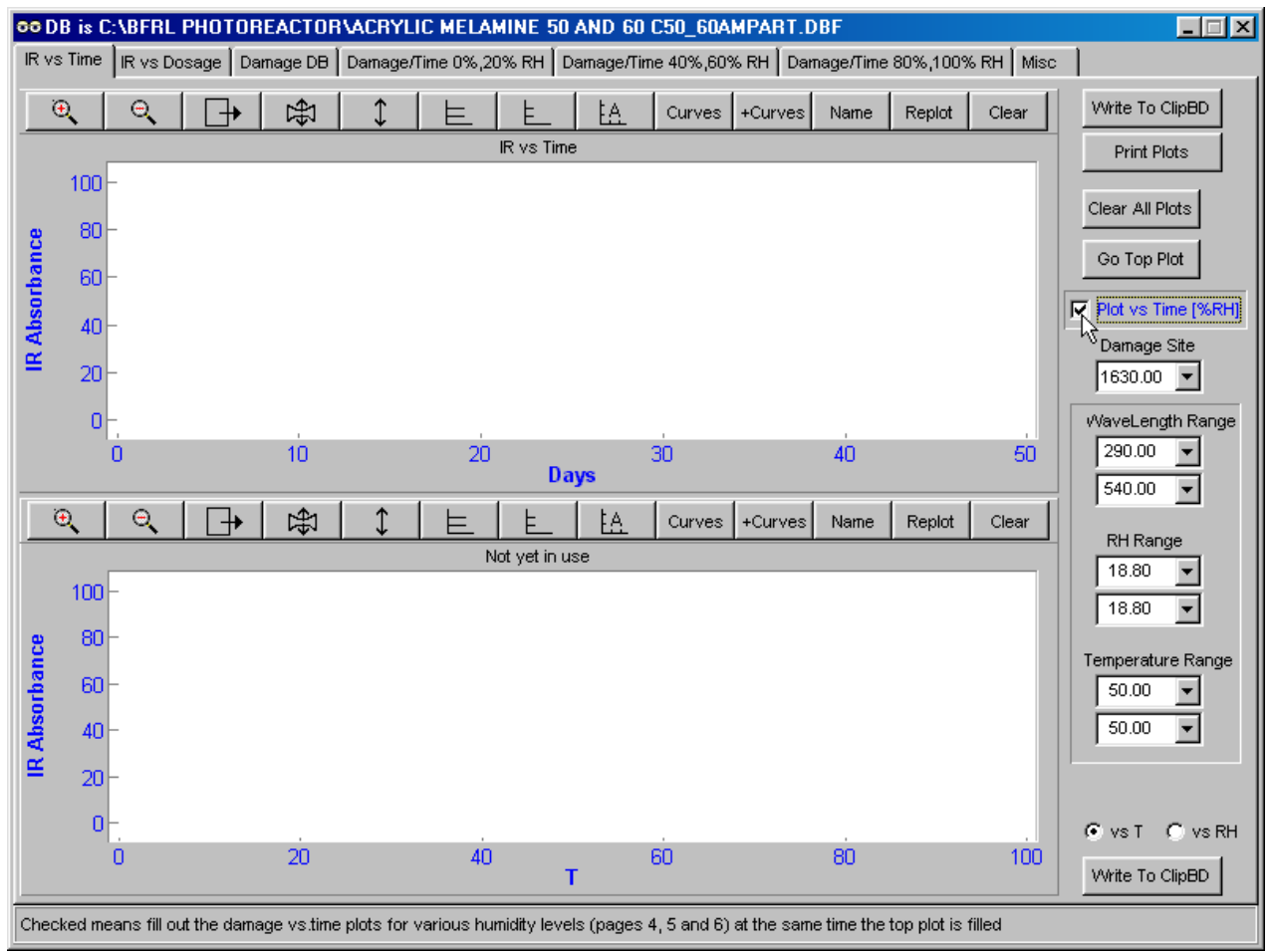

To fill the plots with the information from the damage database table for the conditions specified, click on "Go Top Plot". However, only one level of $\mathrm{RH}$ has been specified, so the program can not interpolate/extrapolate as needed to estimate the damage at various levels of $\mathrm{RH}$ and the warning results:

When a range of $\mathrm{RH}$ is chosen from the drop down list of those $\mathrm{RH}$ levels available in the database table: 


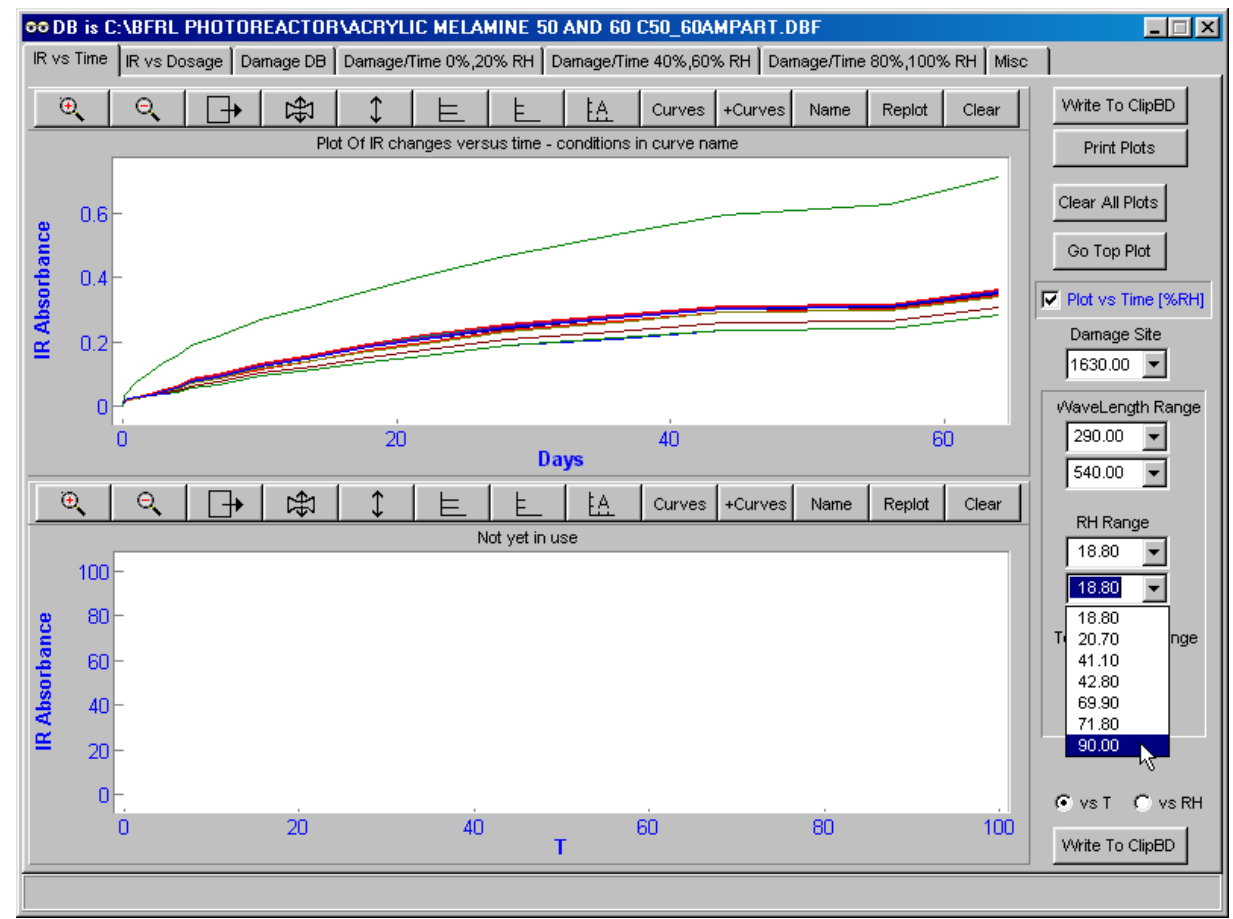

and the "Go Top Plot" button is clicked, the data are plotted:

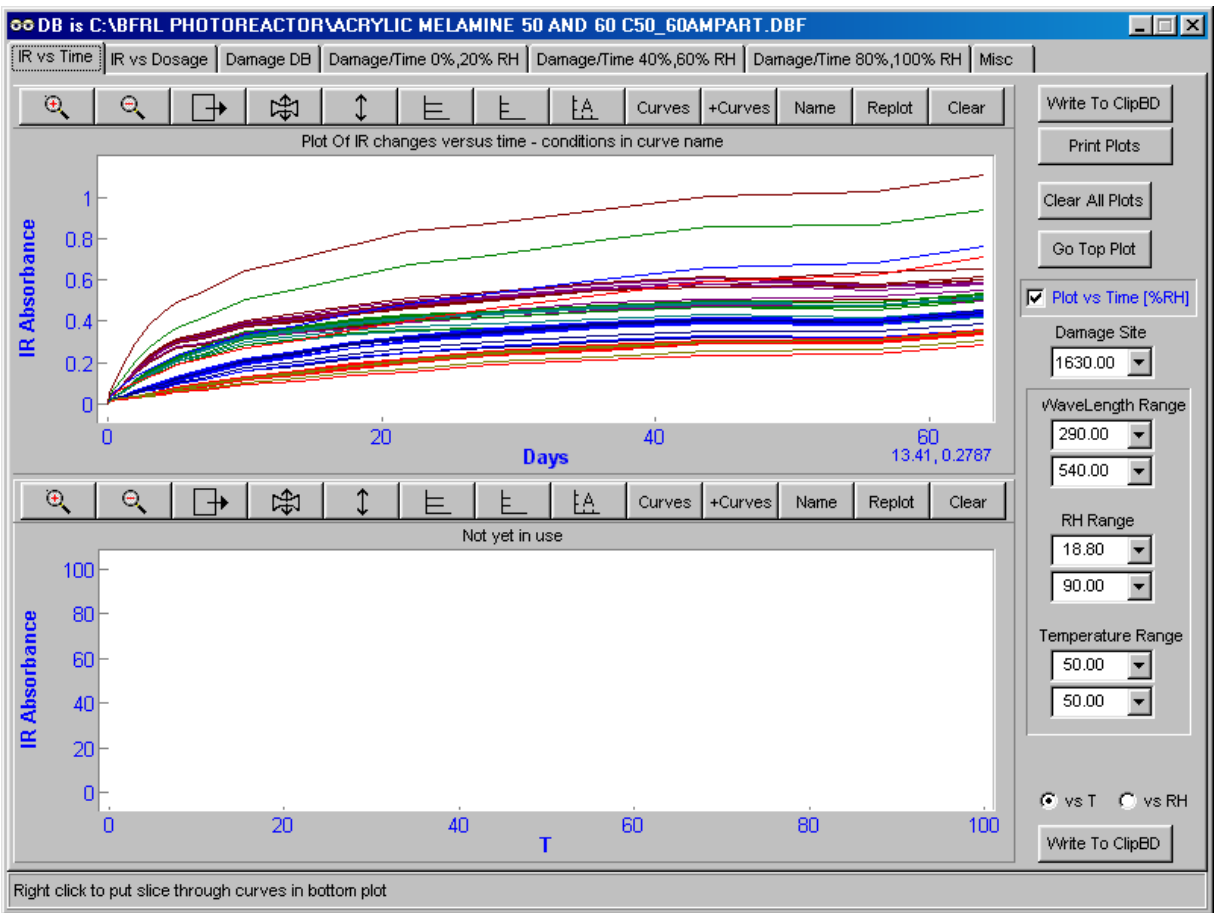


A plot of dosage versus time is also obtained (on page 2).

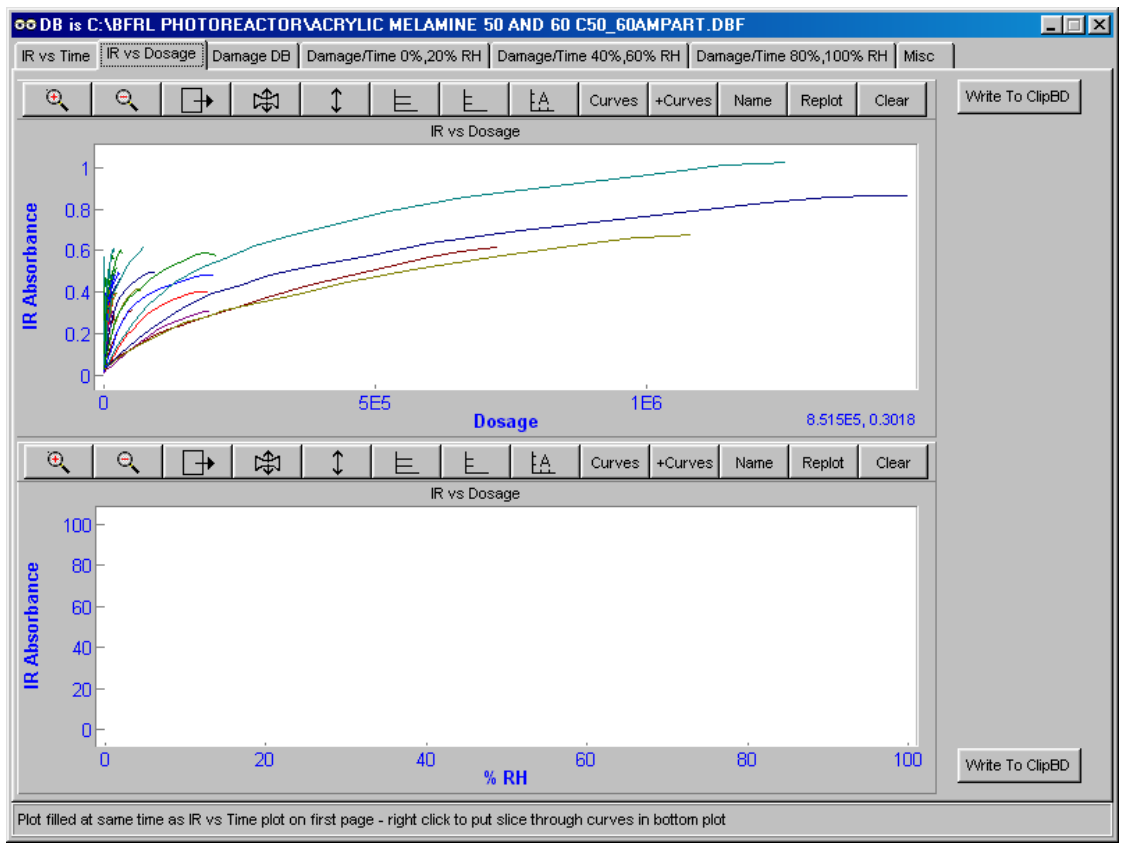

The widely varying levels of dosage corresponding to the various filters, filter widths, lamp irradiance and specimen absorbance are clearly evident in this plot.

The contents of the damage table may be examined on page 3 , the "Damage DB" page of the program. . The database table can be changed using the "Different DB" button. The "Delete Current Record" button deletes the selected record (currently at the bottom of the table, as shown by the arrow head on the left hand side of the table) from the table by marking the record appropriately, and the "Pack DB" button removes all trace of the record from the table. Until the table is packed, the record can in principle be recovered.

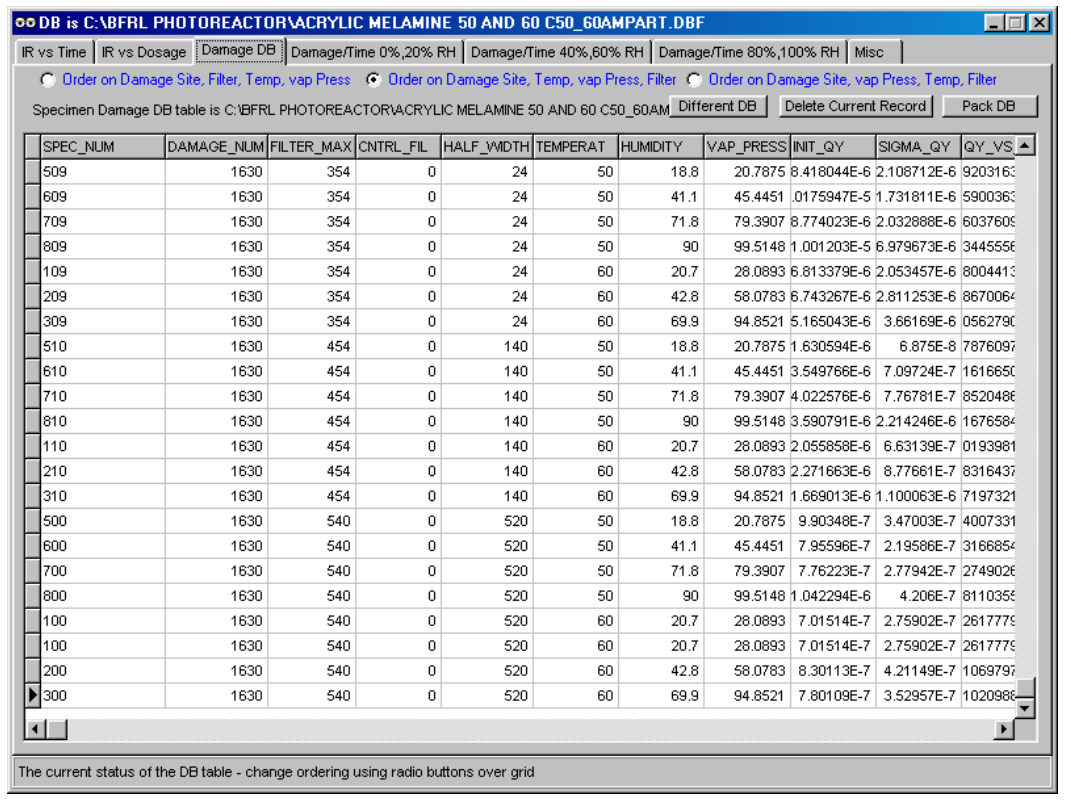


Various values can be read directly from the table. The columns can be resized by dragging the dividing bars on the title row. The table can be moved up and down by clicking on the scroll bar column. The behavior of the scroll bar is "incomplete" because the primary control over the table is the filter imposed on showing the columns and the scroll bar does not know what the logical order of the records is.

Page 4 shows the first of the damage versus time curves for fixed levels of relative humidity and for the temperatures found in the damage table.

Up to now, only one temperature level has been specified and there is only one curve in each plot. The temperature is in the name of the curve, obtained by first putting the plot in "name" mode (the button over the plot is depressed) and right clicking near the curve of interest.

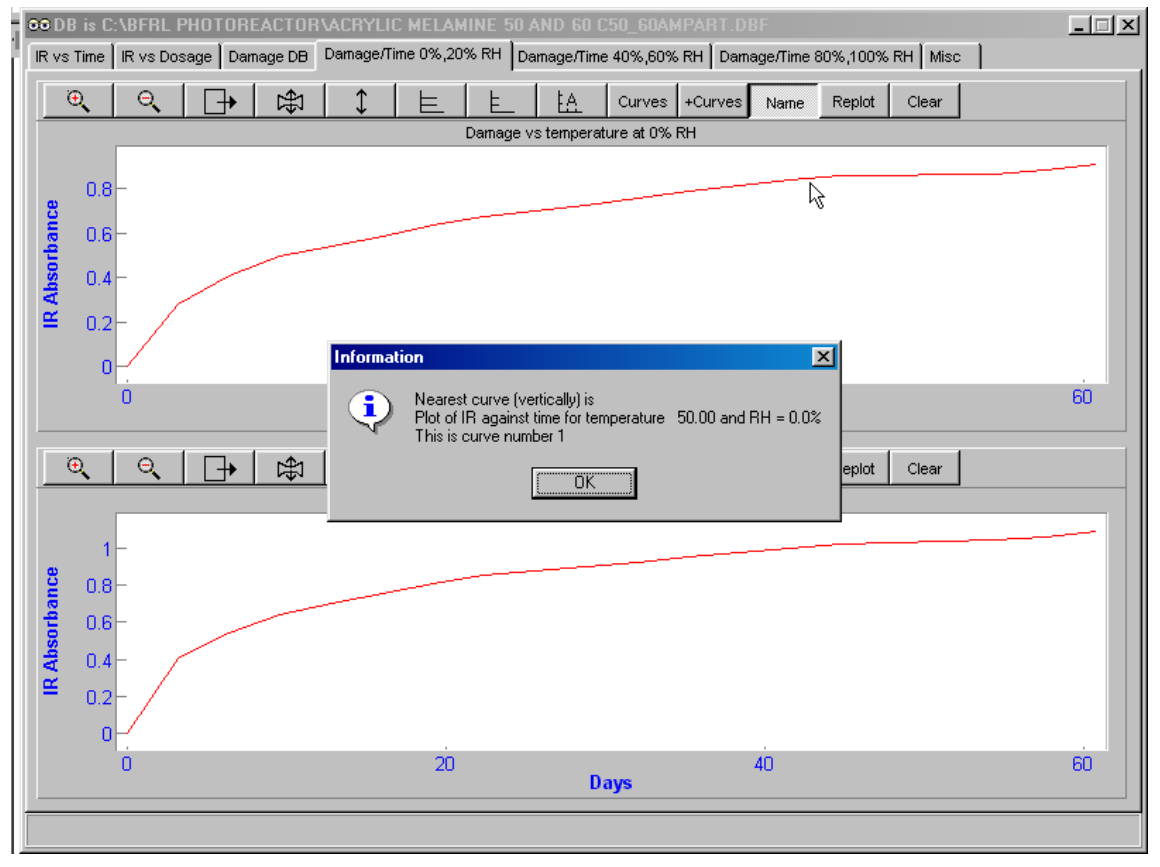

If data for more than one temperature are specified, 


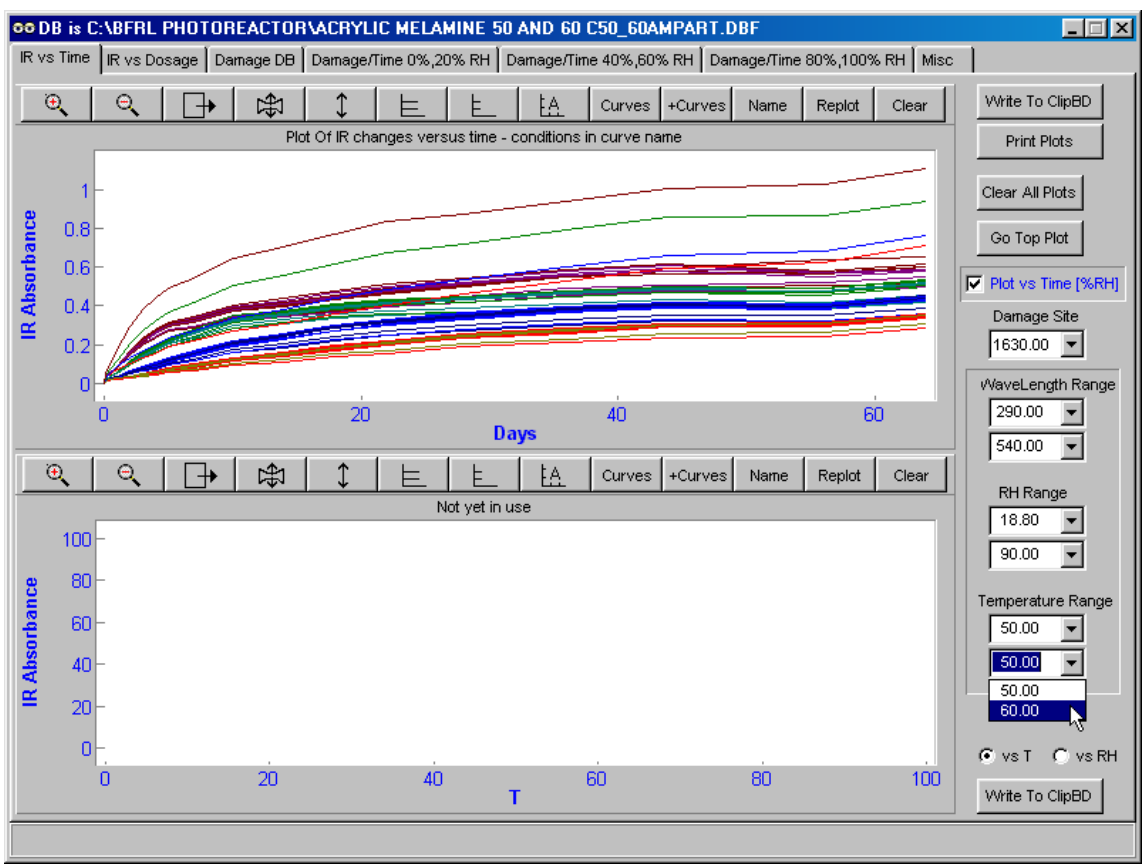

the plots are cleared and Go Top Plot is again clicked:

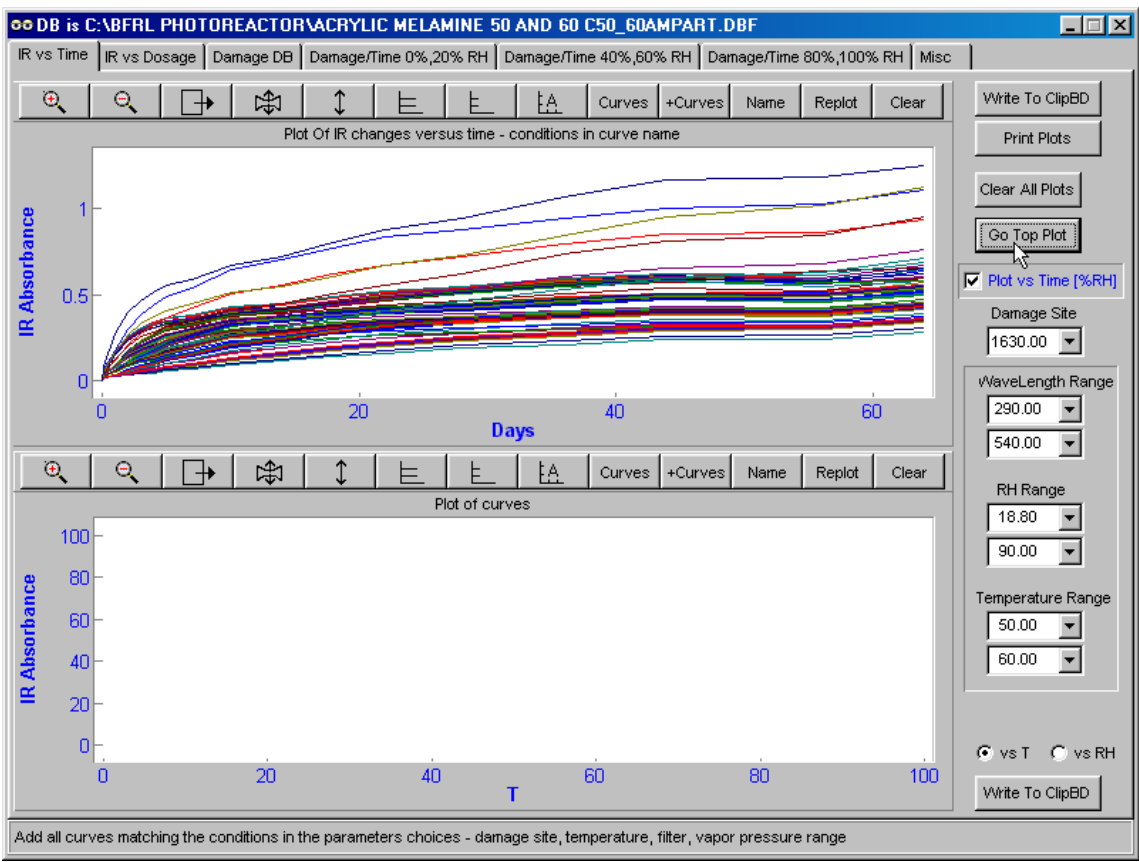

there are now two curves, one for each temperature, in the damage versus time at fixed $\mathrm{RH}$ plots. 

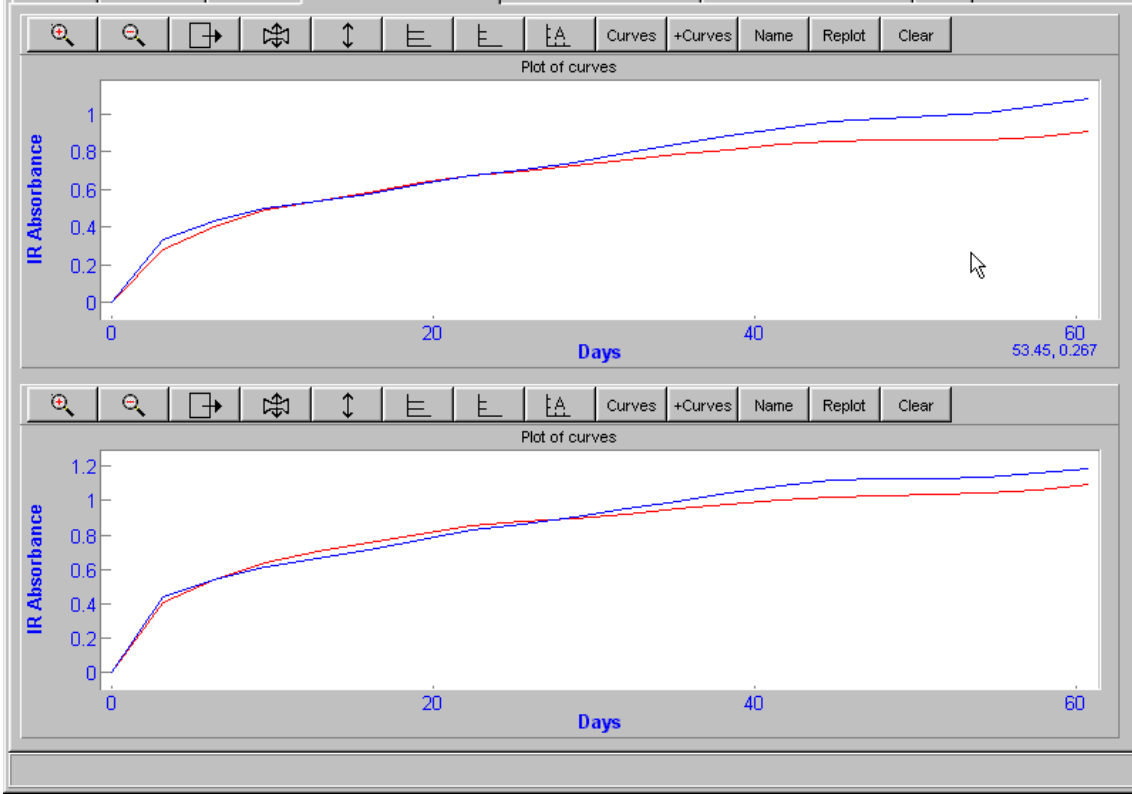

Various other operations can be carried out on the curves. For example, selected curves can be averaged by clicking on the "Curves+" button over the plot (Curves+ is used as a name because there are too many options for them all to fit on the curves button). In this case, one would select the "Average Curves" radio button and then click on the OK button.

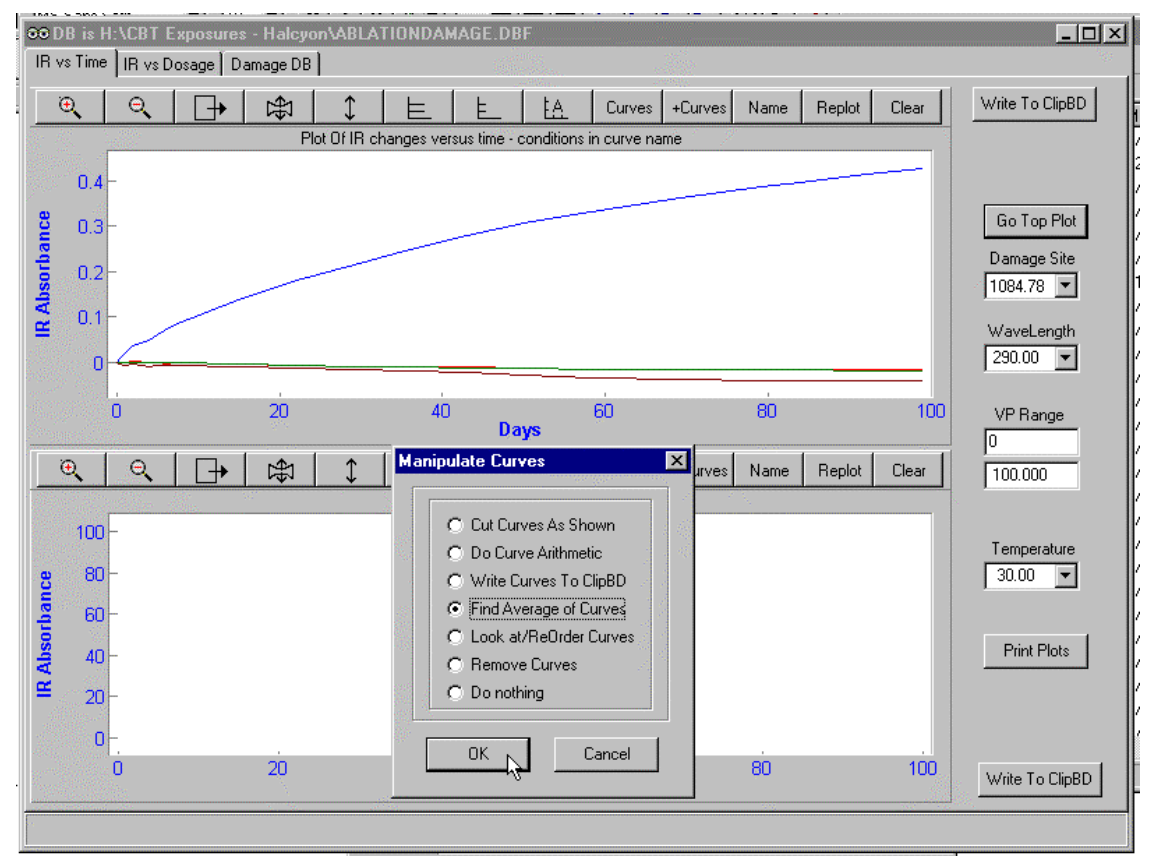

A dialog box appears containing the curve names in the colors of the curves (the colors repeat every 8 colors - the range of colors is restricted because the colors have to show well on a white background - whether 
on the screen or on paper). The curves to average together are selected by checking the appropriate check boxes.

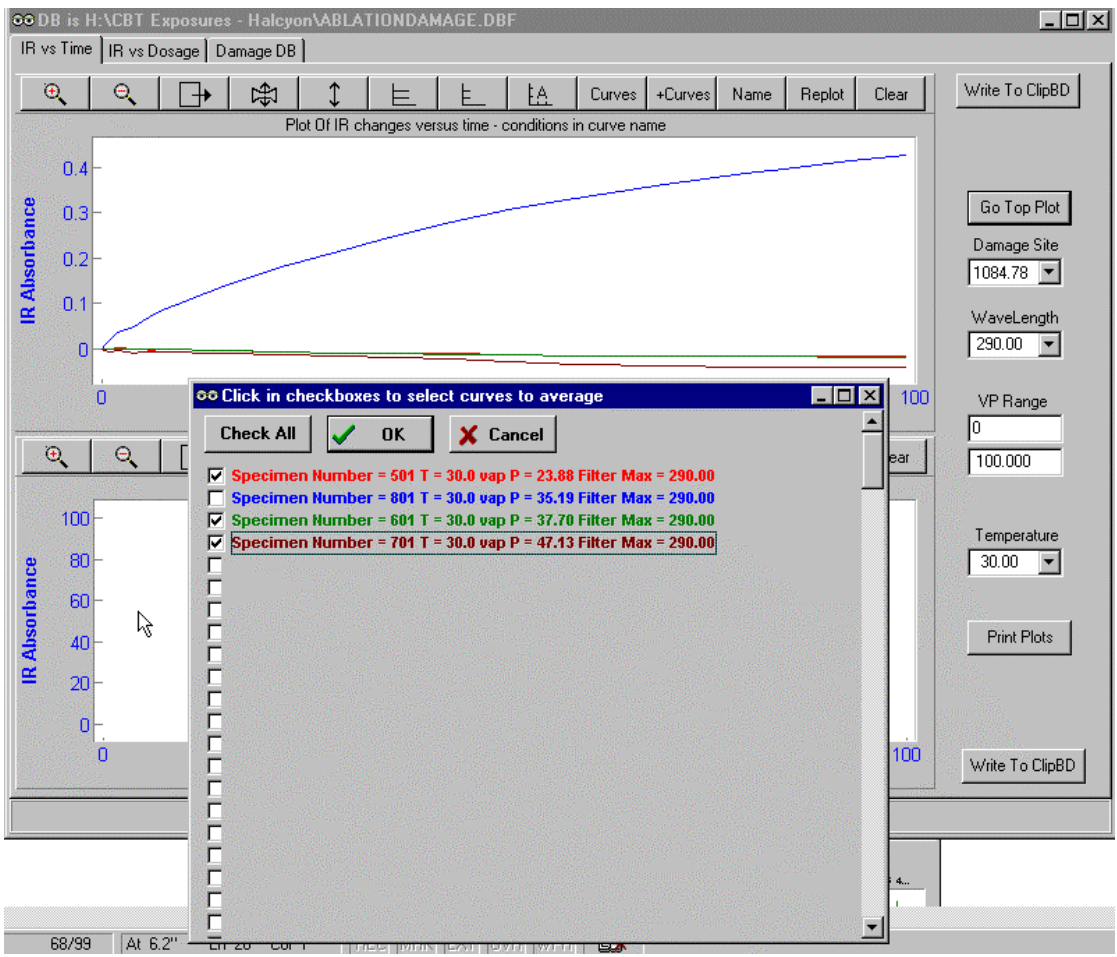

Clicking on the OK button plots the average of the selected curves and also boundary curves with +1 standard deviation (of the average, not the population) and -1 standard deviation. These curves can easily be deleted by invoking the same curves+ button dialog, selecting "Delete curves" and checking the appropriate check boxes for deleting. 


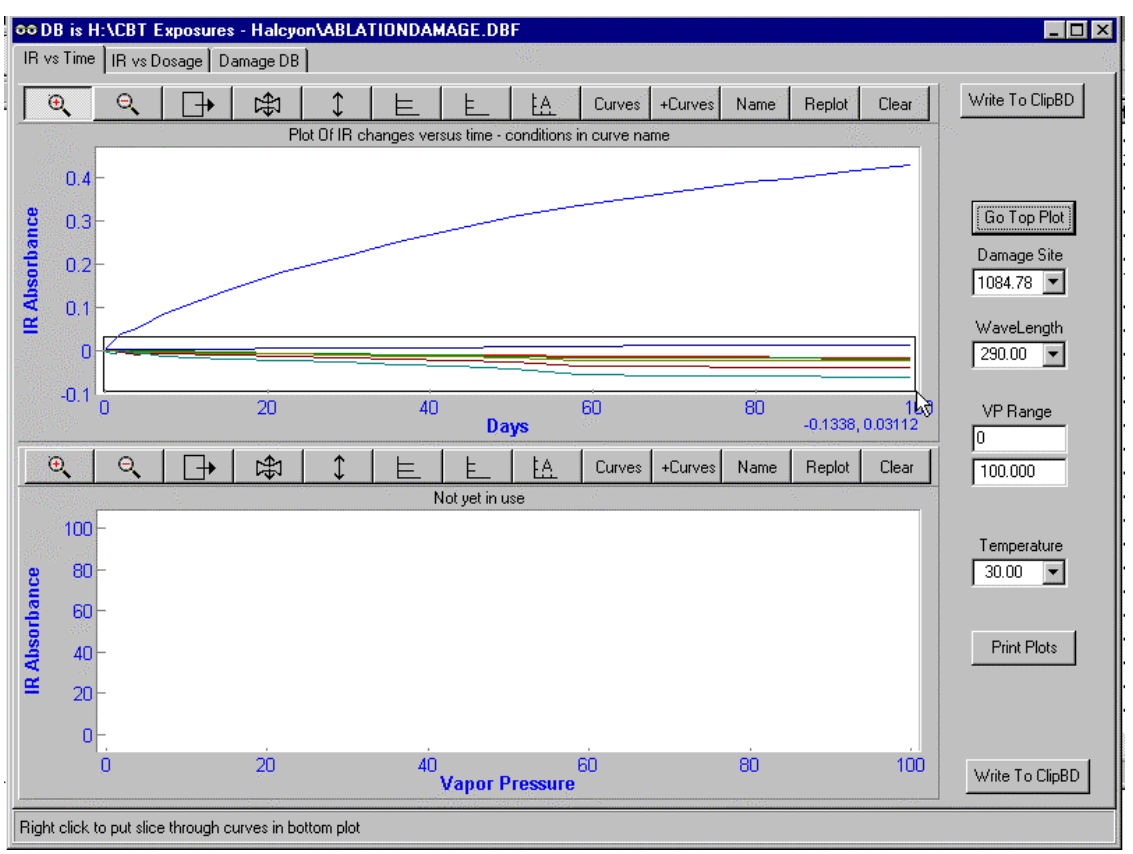

The identities of the curves are revealed by setting the plot to names mode and right clicking near the curve of interest, as shown in the next two figures.

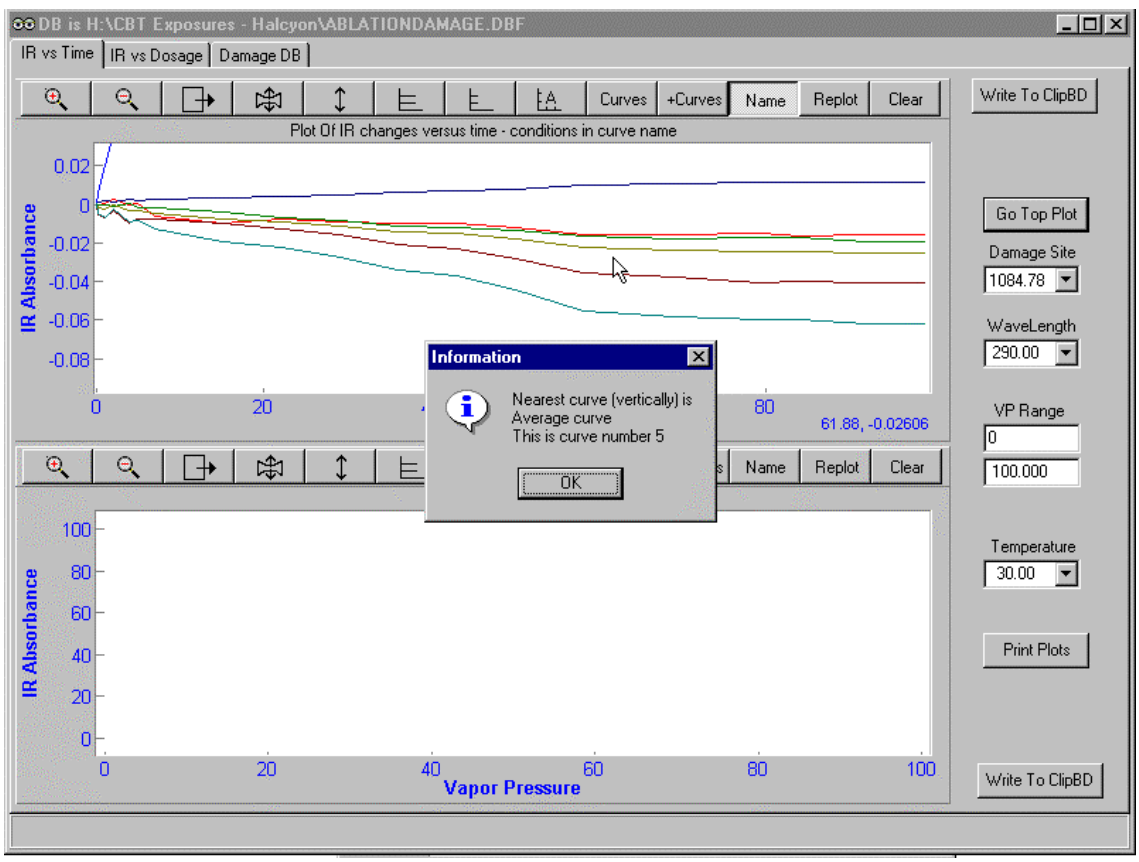




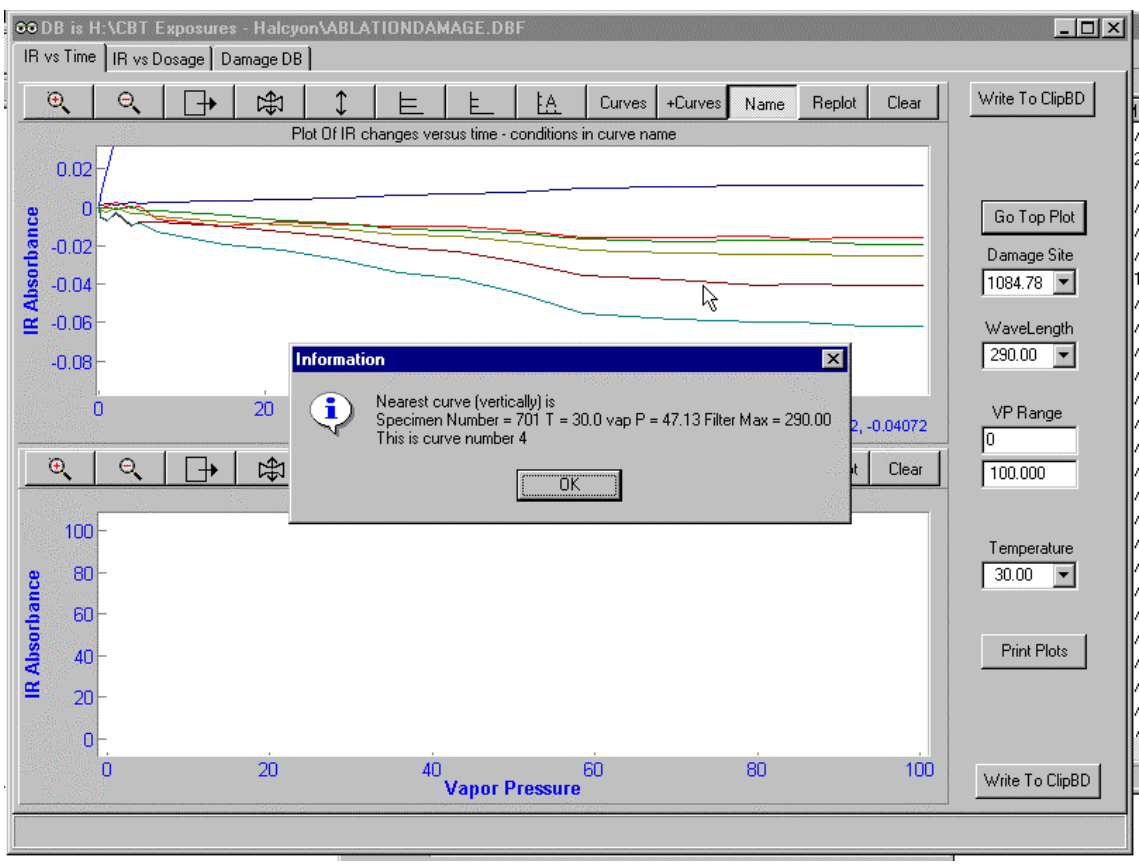

When the plot is not in names mode, right clicking puts a new line in the bottom plot. The line is the damage as a function of vapor pressure at the time clicked in the top plot. This line covers all curves in the top plot. (Curves are added to the top plot each time the "Go Top Plot" button is clicked. The plot is cleared using the "Clear" button over the plot.)

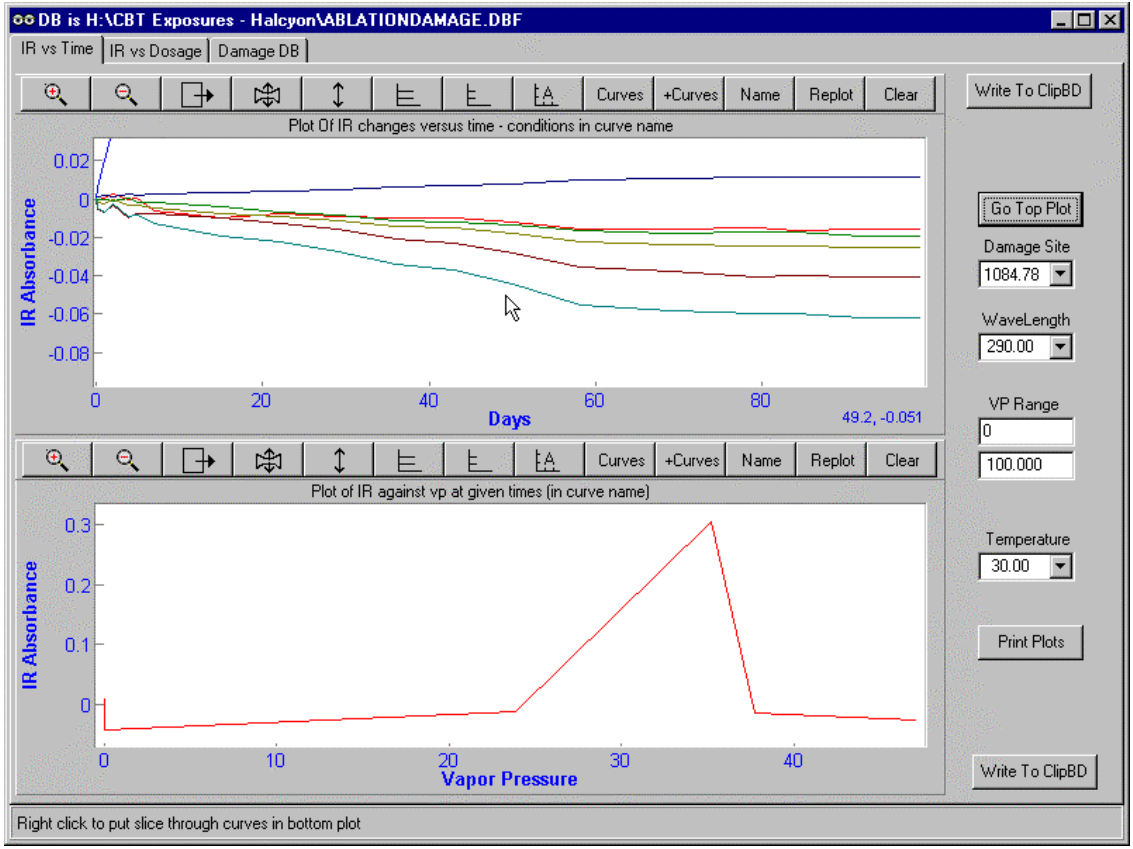

The second page of the program, the "IR vs Dosage" page, plots the damage against dosage rather than days. This plot is more prone to error than the time plot because the dosage requires that the lamp, filter and the specimen UV spectra be well known. The specimen UV spectra in particular are not well-known because the absorbance values are typically very close to zero (i.e., very near the level of the noise of the 
measurements) in some regions of the spectrum - for example in the visible region for clear coatings.

The top plot on the "IR vs Dosage" page is filled at the same time as the top plot (damage versus time) on the first page. It must however be cleared separately. Curves are put into the bottom plot by right-clicking on the top plot. These curves are damage versus vapor pressure at a given dosage (they were at a given time in the plot on the first page).

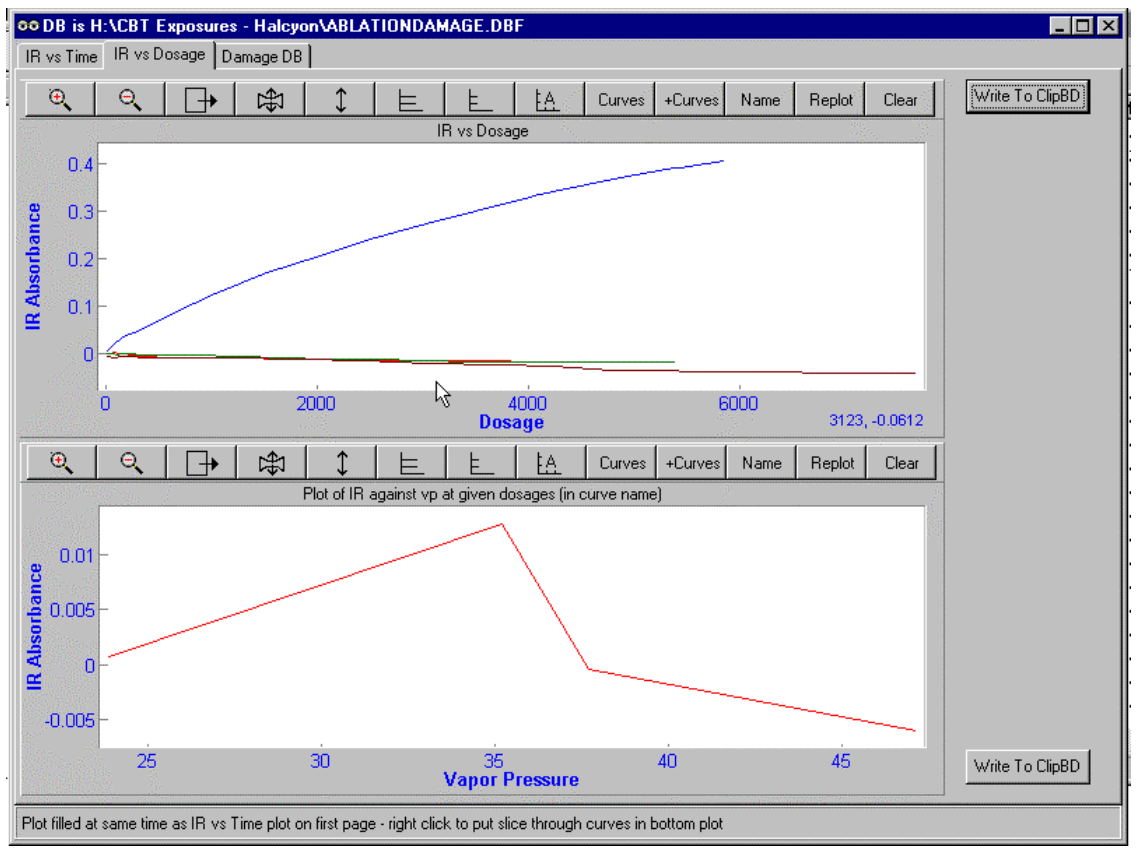

\section{LookAtDamage program}

The Look At Damage program allows the quantum yields and dosage and damage curves to be plotted in various ways. Dosage, damage and quantum yields are calculated using the AbsorbedUVvsIR.exe data processing program. To speed up accessing the data and to allow some condensation of data in various directories, the needed data are copied into the damage database table from the parent tables as each record in the damage table is written. This is greatly advantageous in the case of the damage spectra, for example, which have undergone processing which depends on the control parameters chosen by the user. Regenerating the results from the original data as needed would be very time-consuming.

When the program begins, the database table must be specified. The table is read and the experimental parameters are stored in drop down list boxes. 


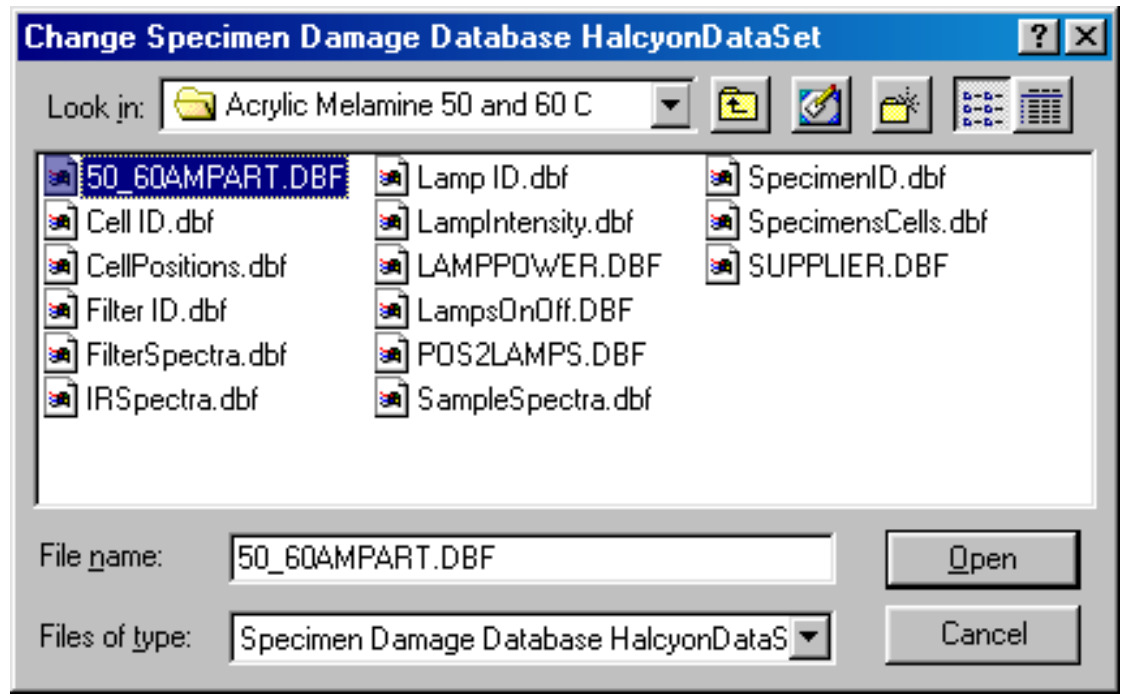

The database table, from page 4 of the program, is shown below.

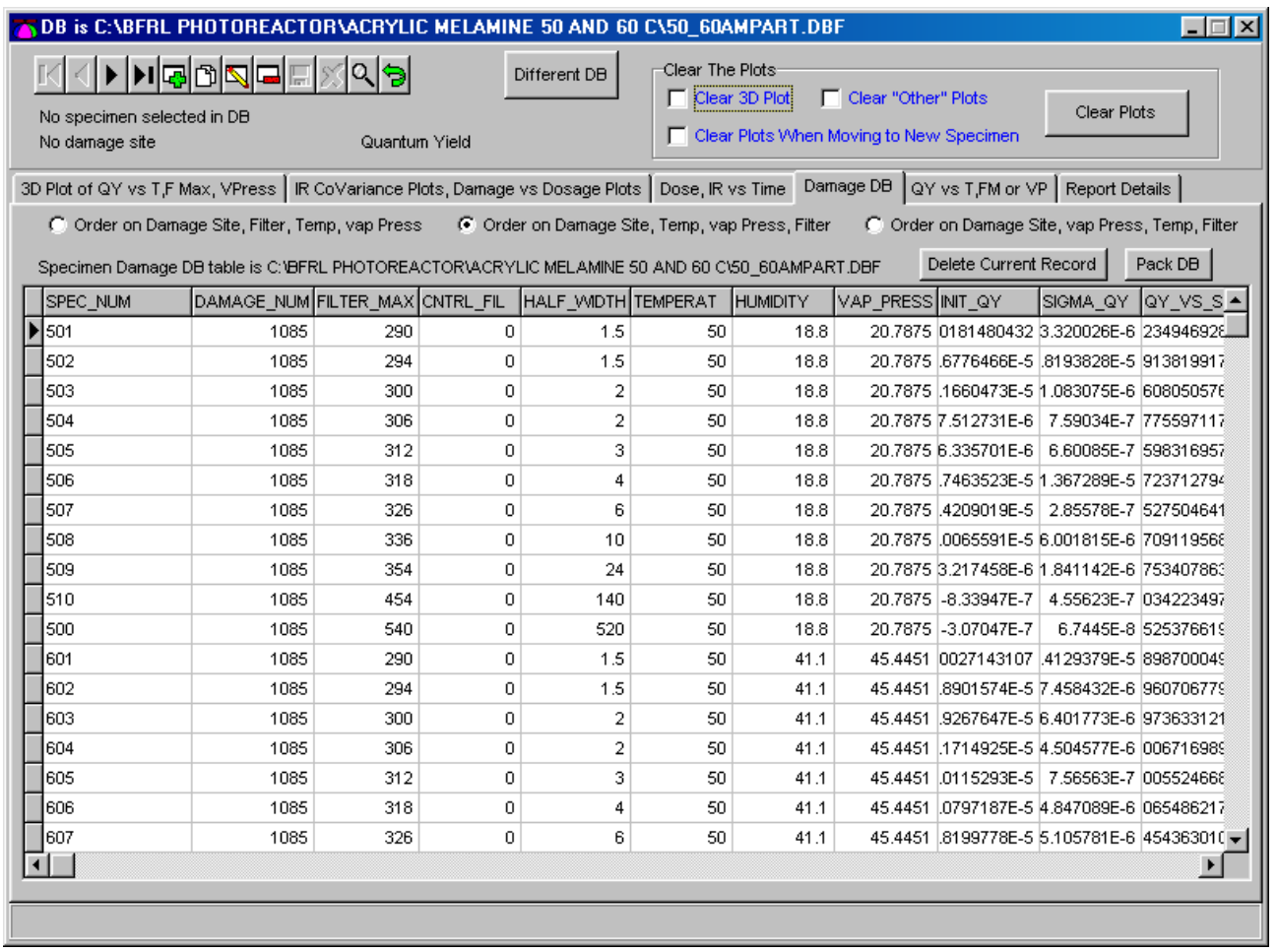

Note that the columns can be resized by dragging the column heading dividers as shown below. 
The database table, with resized columns. The caret at the left hand side of the table shows the current record. If the navigator bar at the top left of the screen (below) is used to step through the table, data from the table can be entered into the plots on the first three pages of the program.

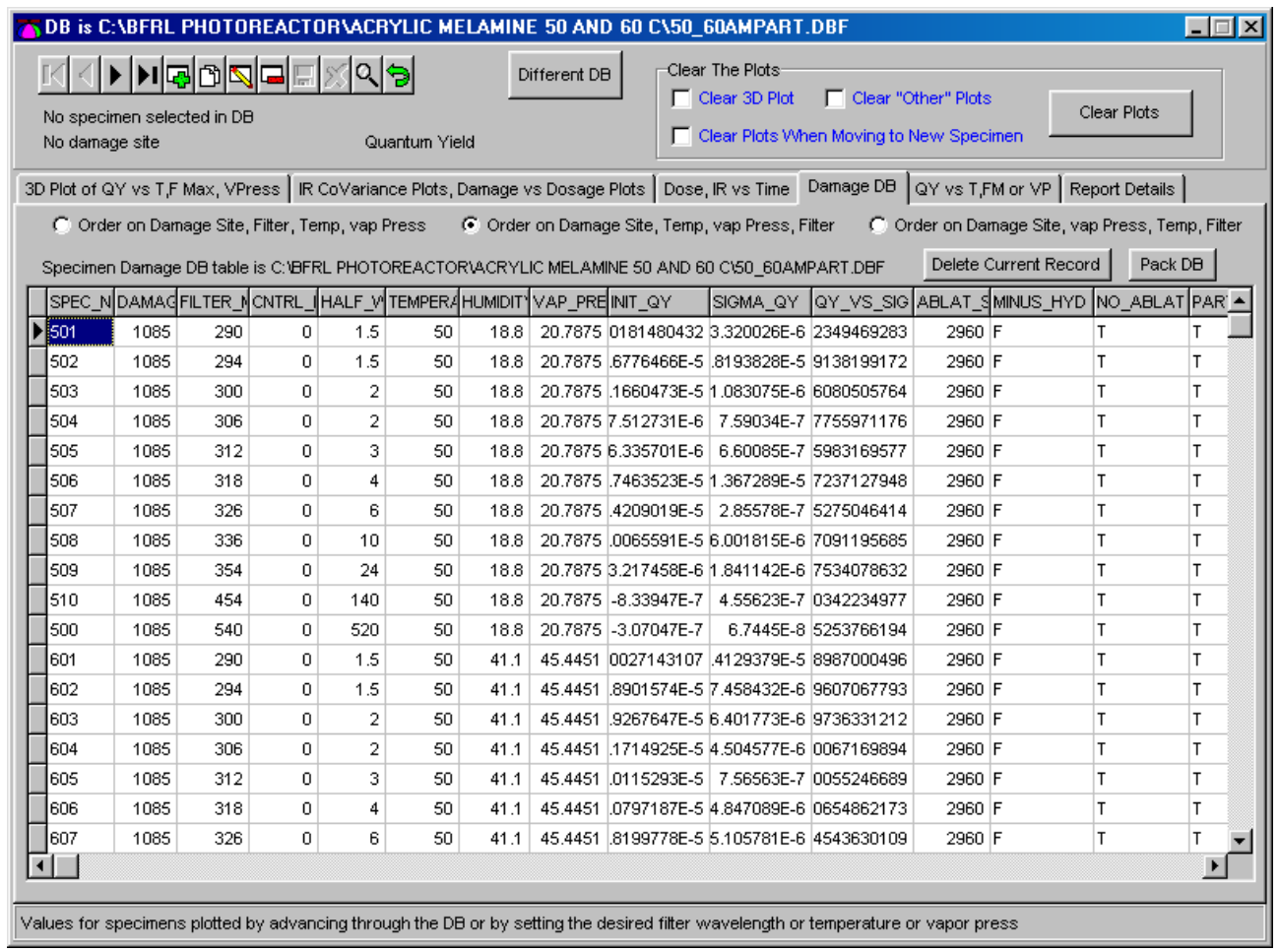

Plots of dosage versus time (top plot) and damage versus time (bottom plot) for the current record in the damage database table are shown in the next figure. 


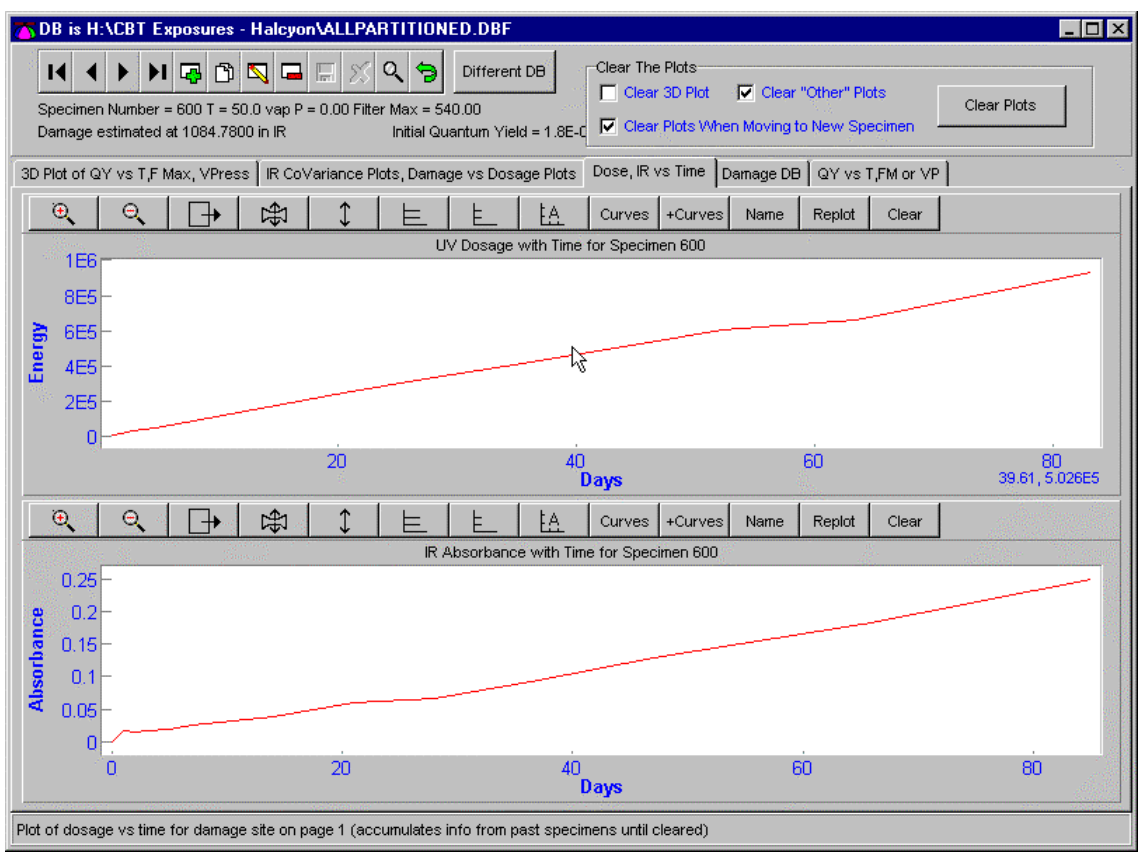

On page 2 of the program, the top plot contains the correlations of all other parts of the damage spectrum with the part (wavelength) at which damage was assessed.

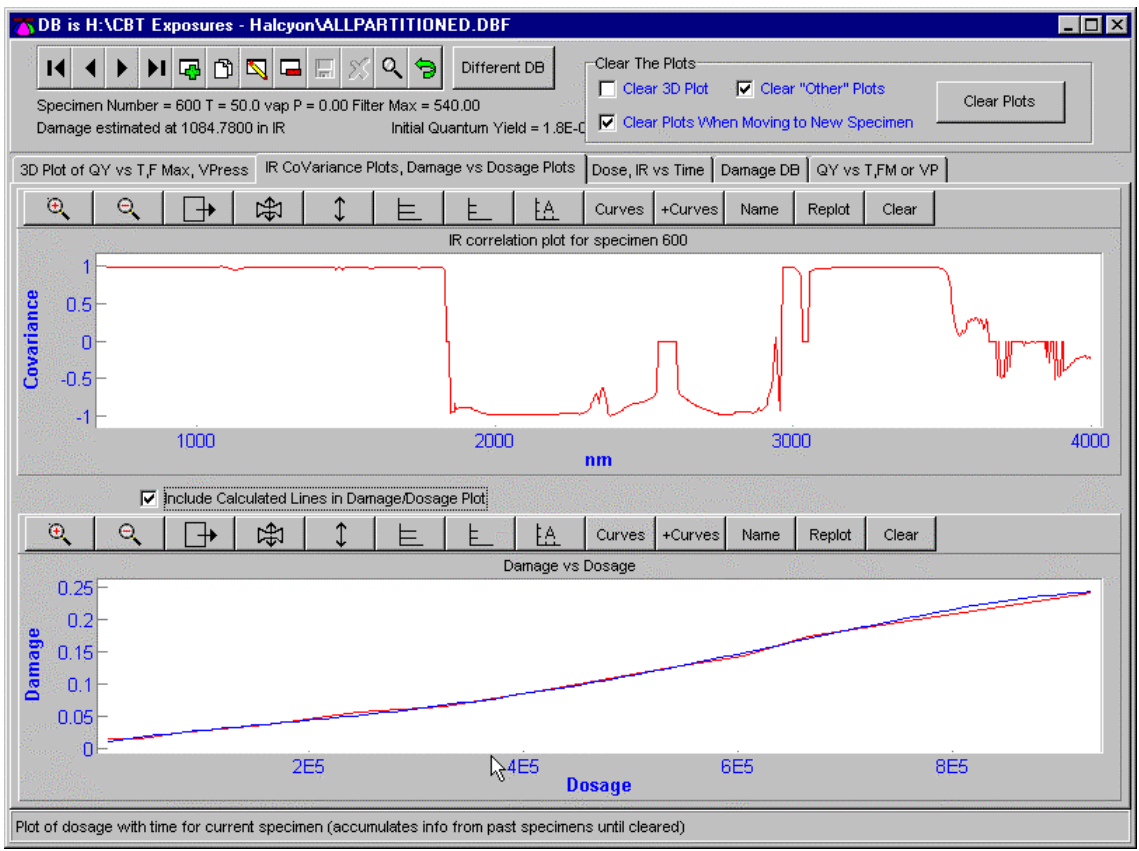

The bottom plot is the damage dosage relationship. The quantum yield is the slope of curve in the bottom plot. The red line in the bottom plot is the observed damage/dosage relationship, the blue curve is the fitted polynomial. (These lines are only red and blue when they are the first 
and second curves in the plot. Curves added later have contrasting colors.)

The first page of the program is a 3-D plot of quantum yield versus filter wavelength (vertical), temperature (horizontal), and vapor pressure (this axis is directed "into" the screen). If the "Plot Quantum Yields in 3D" check box is checked, values are entered into the plot as the program progresses through the damage table following clicks on the navigator bar. The exposure conditions for the currently selected specimen in the damage table are displayed.

Choose Damage Wavelength
Specimen Number $=600$ Temperature $=50.0$ vap Press $=0.00$ Filter Max $=540.00$
Quantum yield green up to this limit 1.0000000
Quantum yield red beyond this limit
2.0000000

Since three dimensions have now been used, the quantum yield values are shown in "coded color" in small spheres, where values below the limit of 1.00000 in the above example are shown green, those above the limit of 2.00000 in the above example are shown red, and intermediate values are shown intermediate between green and red. We have not yet had time to explore the feasibility of this type of plot. An example is shown below.

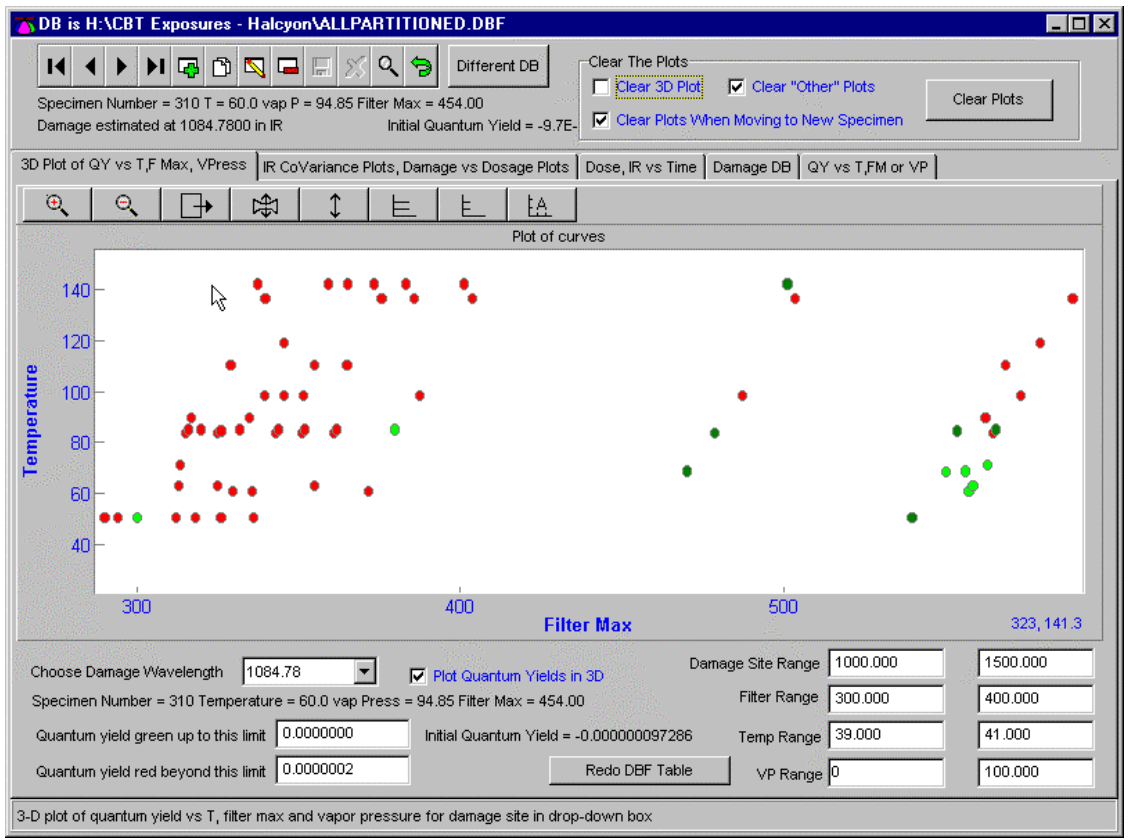

Page 6 of the program provides the capability of plotting the initial quantum versus filter wavelength, temperature, or vapor pressure (as shown in the label of the horizontal axis of the plot) for the conditions 
shown in the drop down list boxes. The navigator bar is not used in making these plots. The initial stage of Page 6 is shown below.

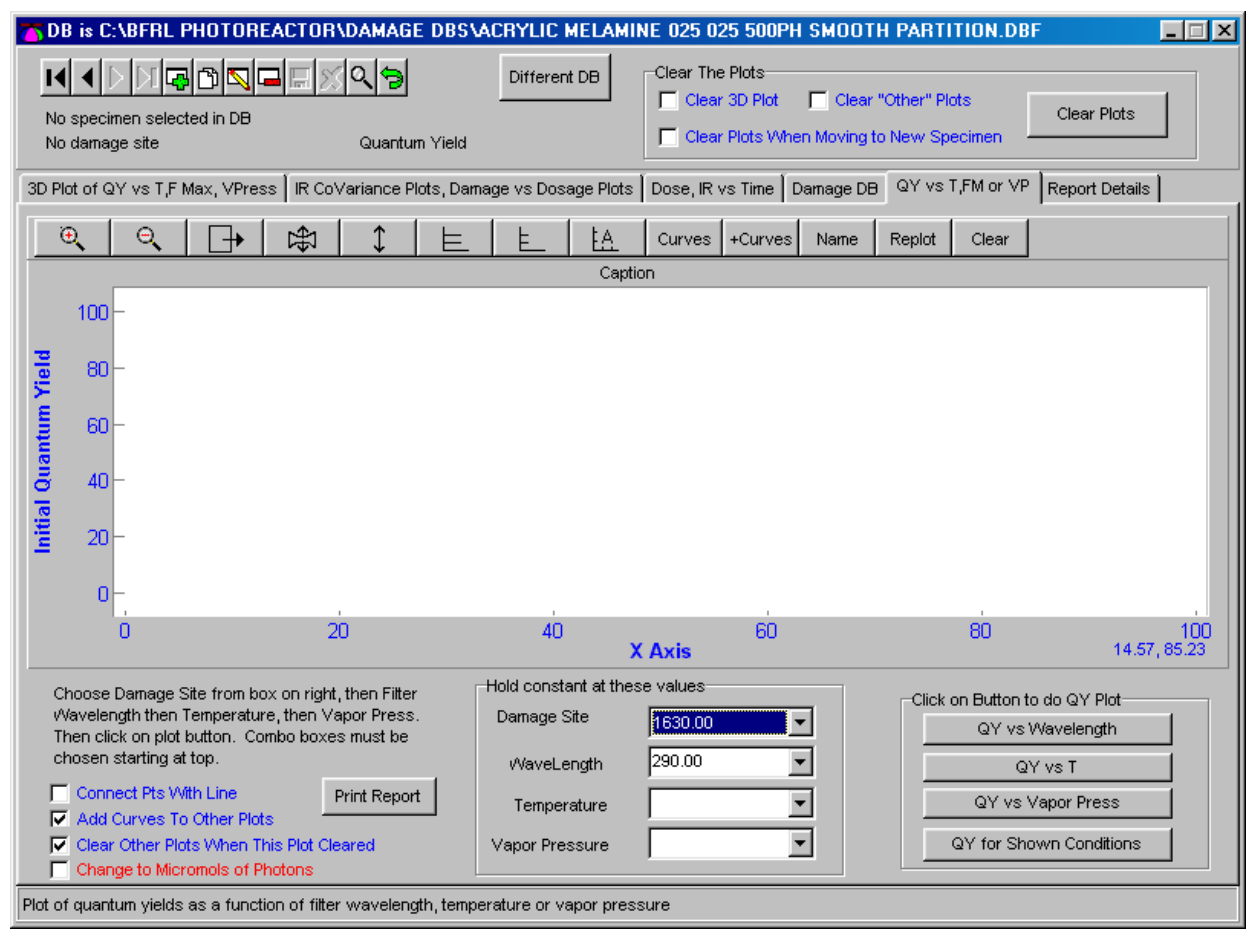

When a damage database table is first read into the program, the damage sites and exposure conditions are read from the table and will later be copied into the various drop-down list boxes so they can be presented as choices.

First a damage site is chosen in the top box. This will put the available filter wavelengths in the second box. One of these wavelengths must then be chosen. This action will put the temperatures available for the chosen damage site and the chosen filter wavelength in the temperature box. Choosing a temperature will put the available vapor pressure levels in the vapor pressure box. This system of filling the drop-down boxes avoids the need to hunt for an available set of conditions.

We have already chosen to examine results for the $1630 \mathrm{~cm}^{-1}$ damage site in the IR spectrum. To fill out the lower drop-down boxes, a wavelength must be selected - only then does the program know the

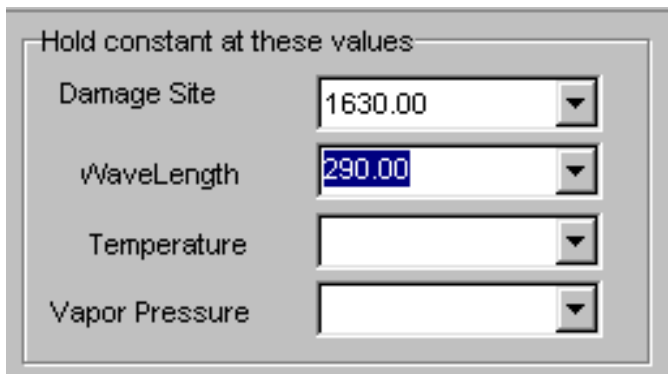

range of available temperatures that it should offer. 
Pull down the drop-down box:

Select a wavelength. Because we are actually going to plot quantum yield against wavelength (we know what we expect the results to look like and can therefore guess how well the procedure has worked), the actual wavelength selected will be unimportant because all wavelengths will be plotted, but it is the mechanism for filling the lower boxes.

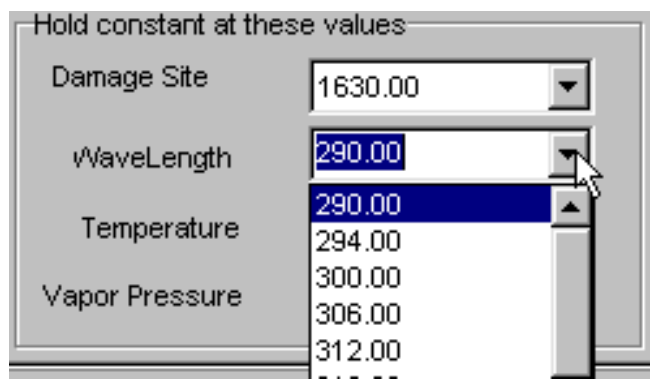

Now we have choices in the temperature and vapor pressure boxes. We select the values we want:

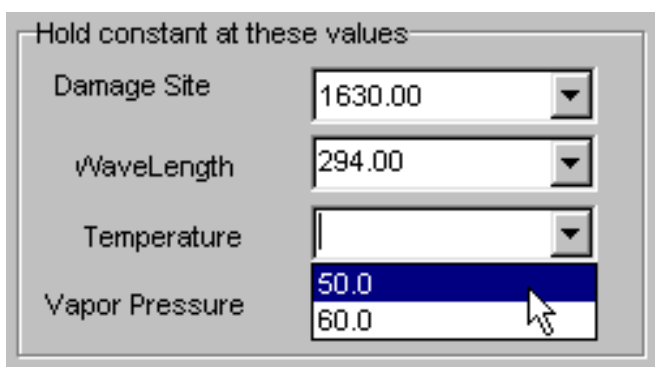

And when we release the cursor, the available vapor pressure values for that temperature will be put in the vapor pressure box.

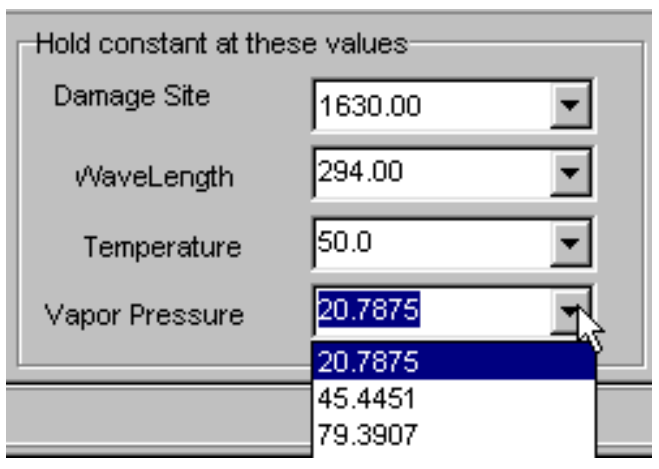


We select a vapor pressure. Actually, the first one is satisfactory because we are eventually going to plot quantum yield for all vapor pressures.

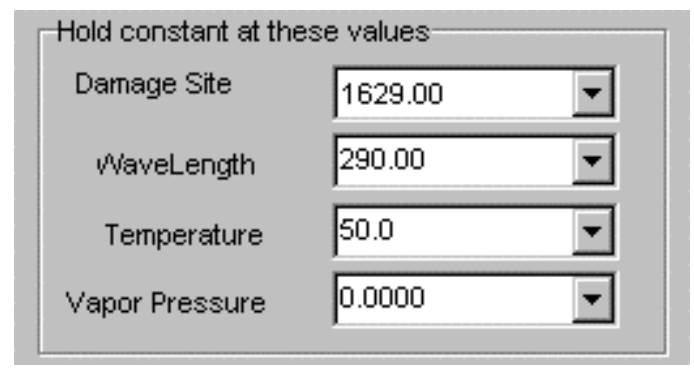

There is probably at most one quantum yield in the table for the set of chosen conditions - that quantum yield can be added to the plot by clicking on "QY for Shown Conditions". Clicking on one of the other buttons

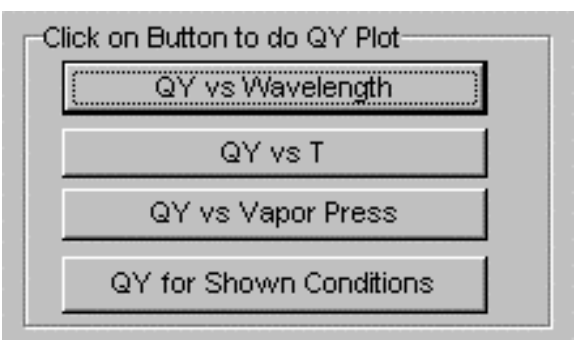

will sort the table according to the criteria mentioned in the button caption and plot the quantum yields. Thus, clicking on the "QY vs Wavelength" will plot as a function of filter wavelength the quantum yields for the damage site which were determined under conditions within the temperature and vapor pressure limits. The plot shown below is then produced.

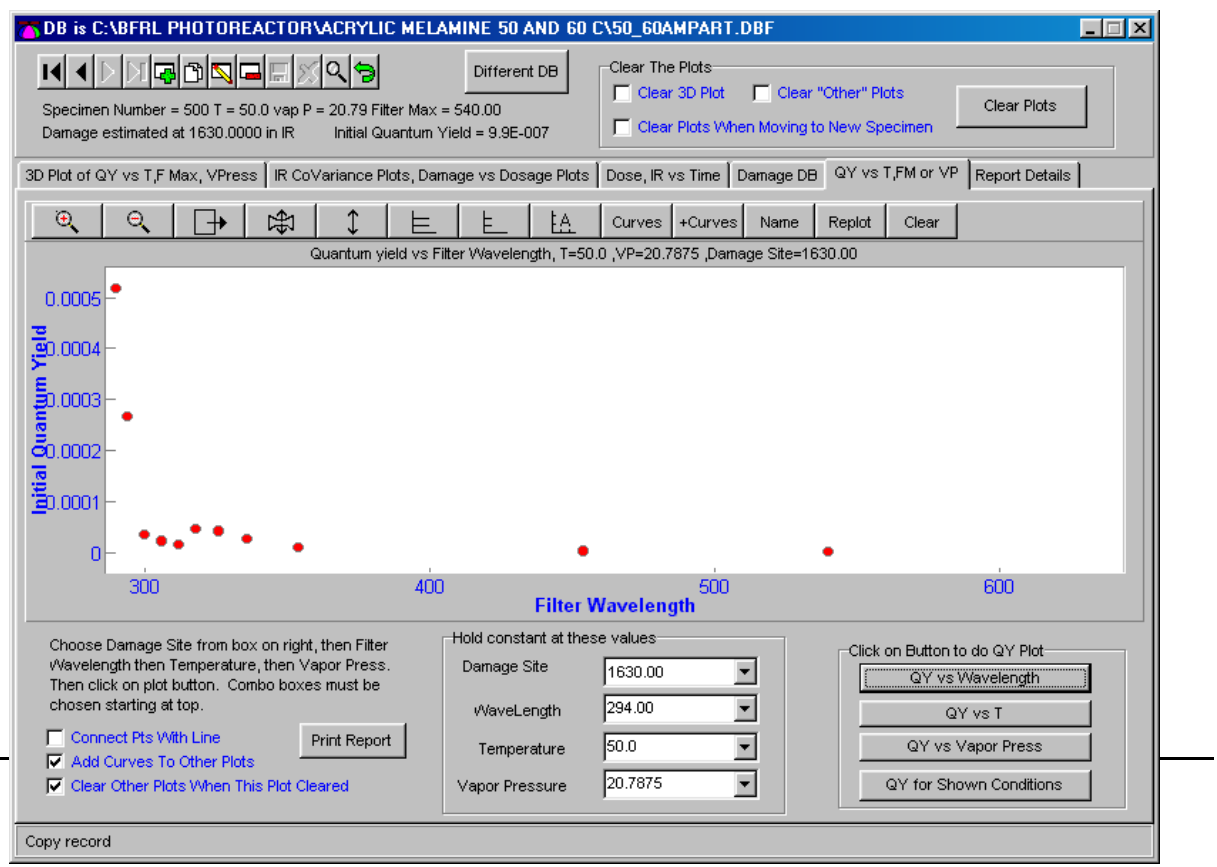


The remaining vapor pressure levels can be chosen then plotted, one at a time, to give the resulting plot:

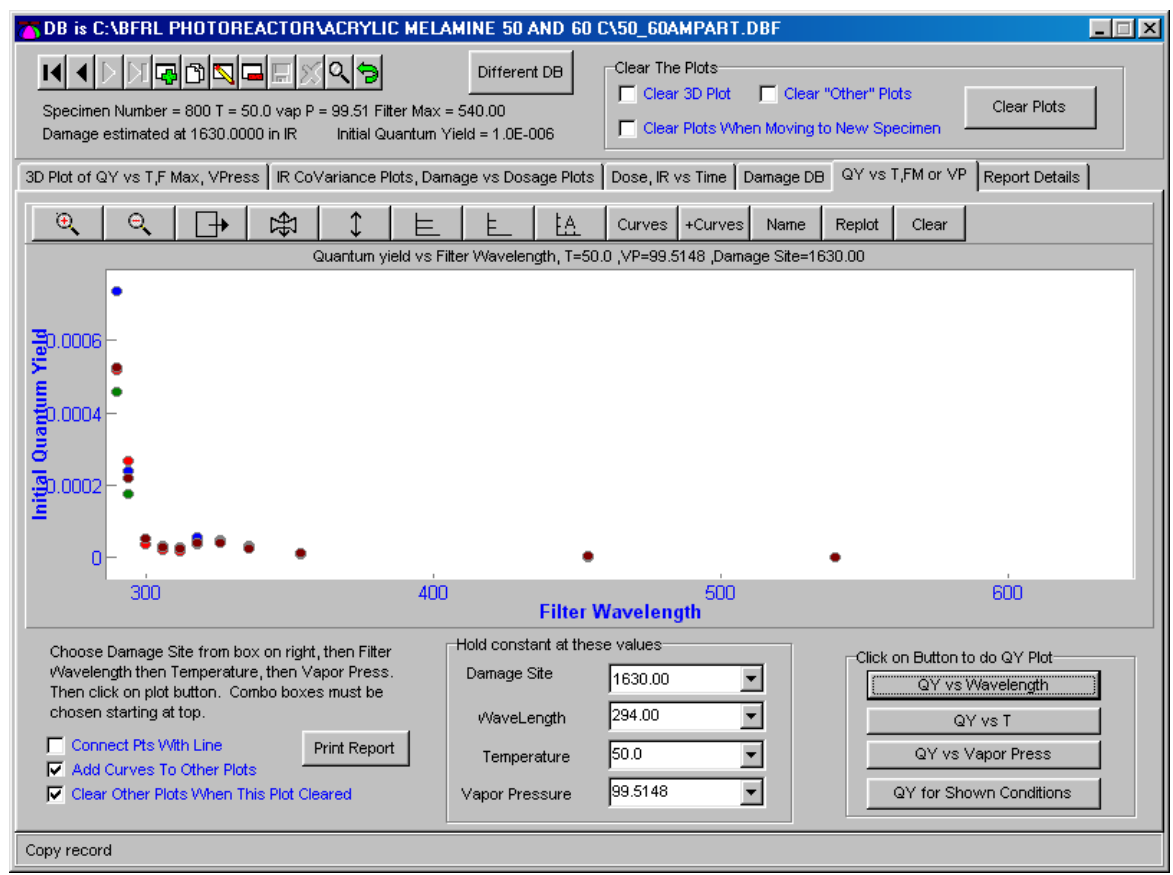

Note that here many additions of sets of quantum yields can be made to the plots. Each set is plotted in a different plot color (the colors cycle every 8 curves/sets of point). The red points above are the first set plotted - the first three colors are red, blue and green. The other colors are mixtures of these primary colors and have non-primary names.

One can say that, given the processing conditions, the data seems to be very consistent with wavelength. The fall-off in quantum yield near 305 $\mathrm{nm}$ is probably because very little degradation occurred because very little radiation passes through the filters at those wavelengths.

The conditions imposed on the data in the program which wrote the damage database table are shown in this screen from the Dosagecalculating program: 


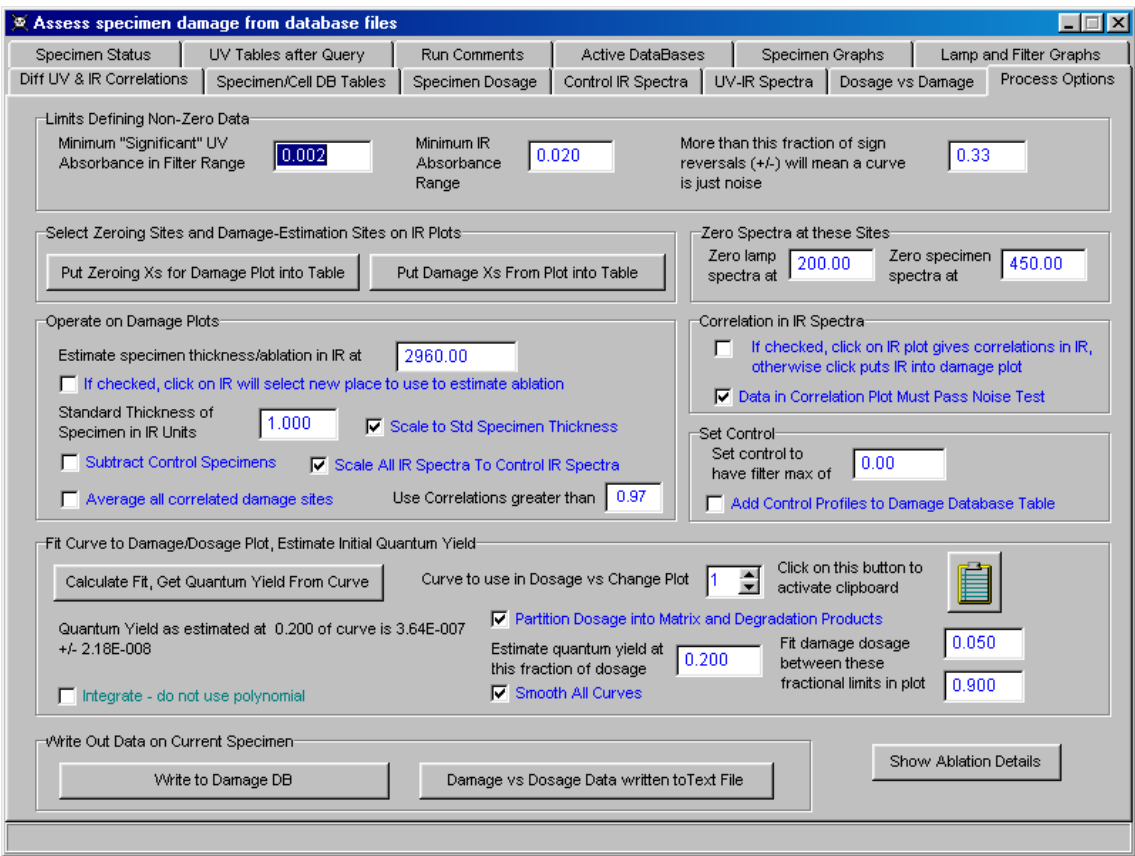

At this point, it was noticed that the data had been calculated without subtracting the control. (If changes are to be ascribed to photodegradation and quantum yields are to be calculated, the dark effects must first be subtracted.) The calculation of damage was repeated and a new damage database table made. The recalculation process took only a few minutes. The corresponding plot of data from that table for four relative humidities at $50 \mathrm{C}$ is shown below. 


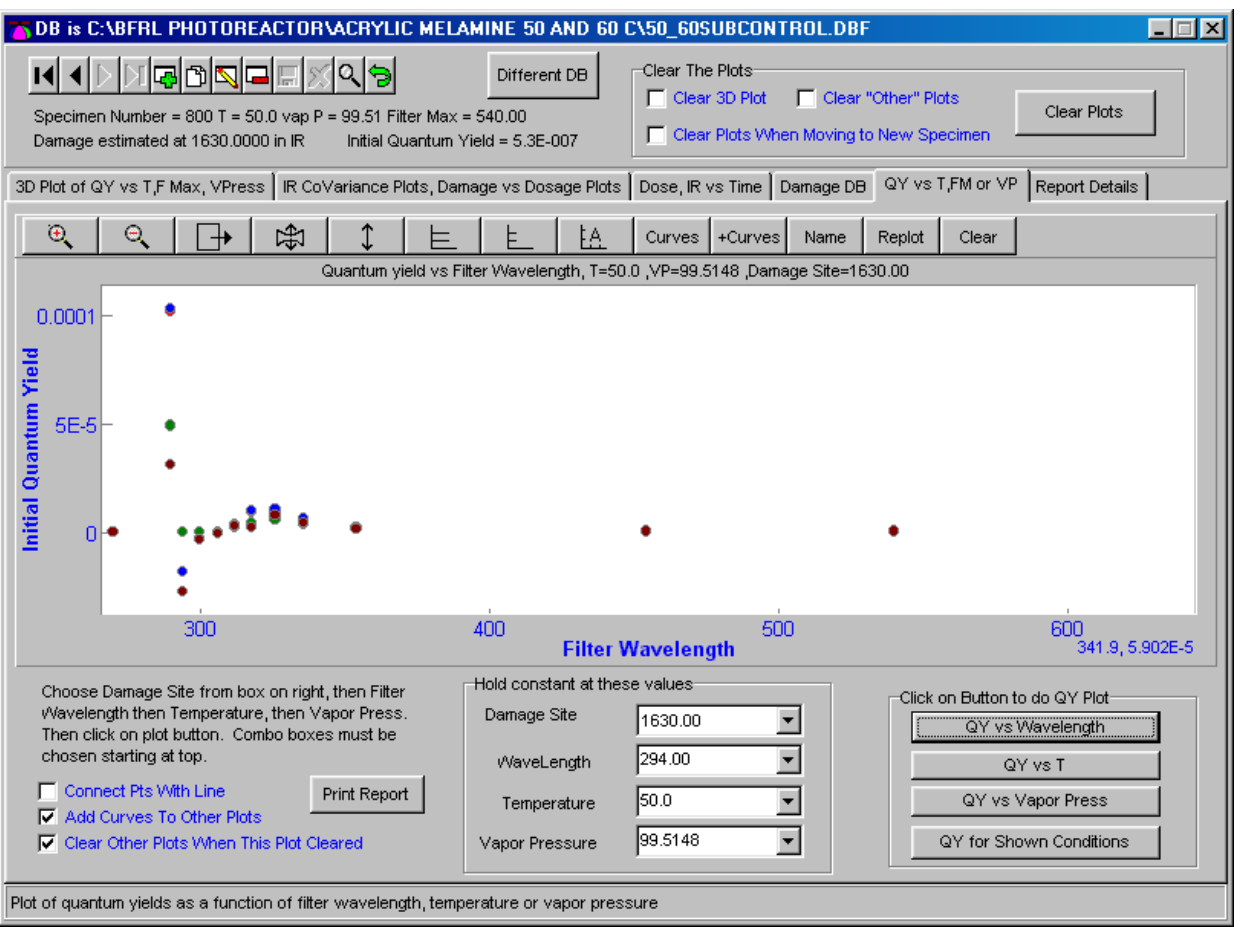

Data for two temperatures and a total of seven levels of relative humidity are shown in the next plot. The quantum values agree very well, except in the region of 290 to $300 \mathrm{~nm}$, where the dosage is very low.

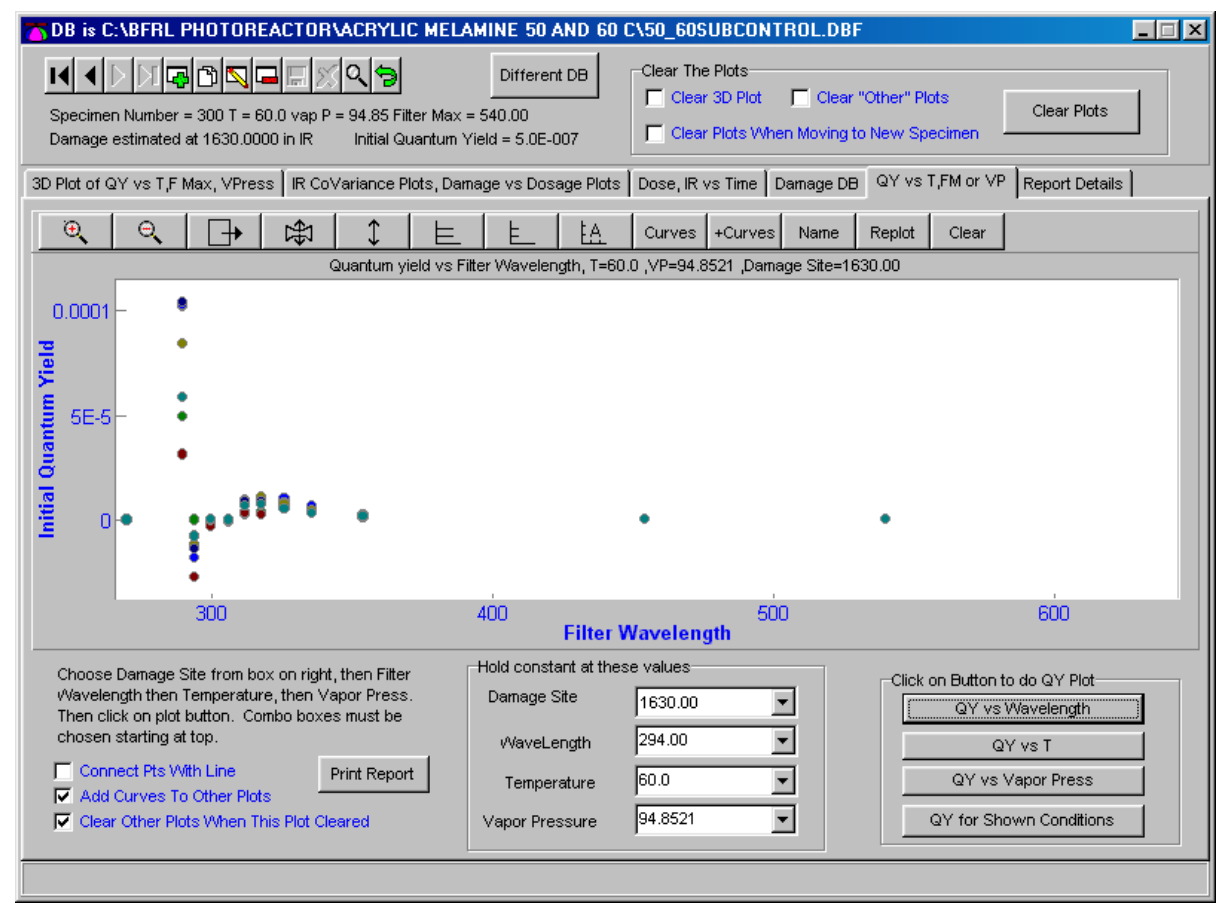


The damage-dosage plot is shown below - there are some cases which have very low dosage, as shown by the short lines.

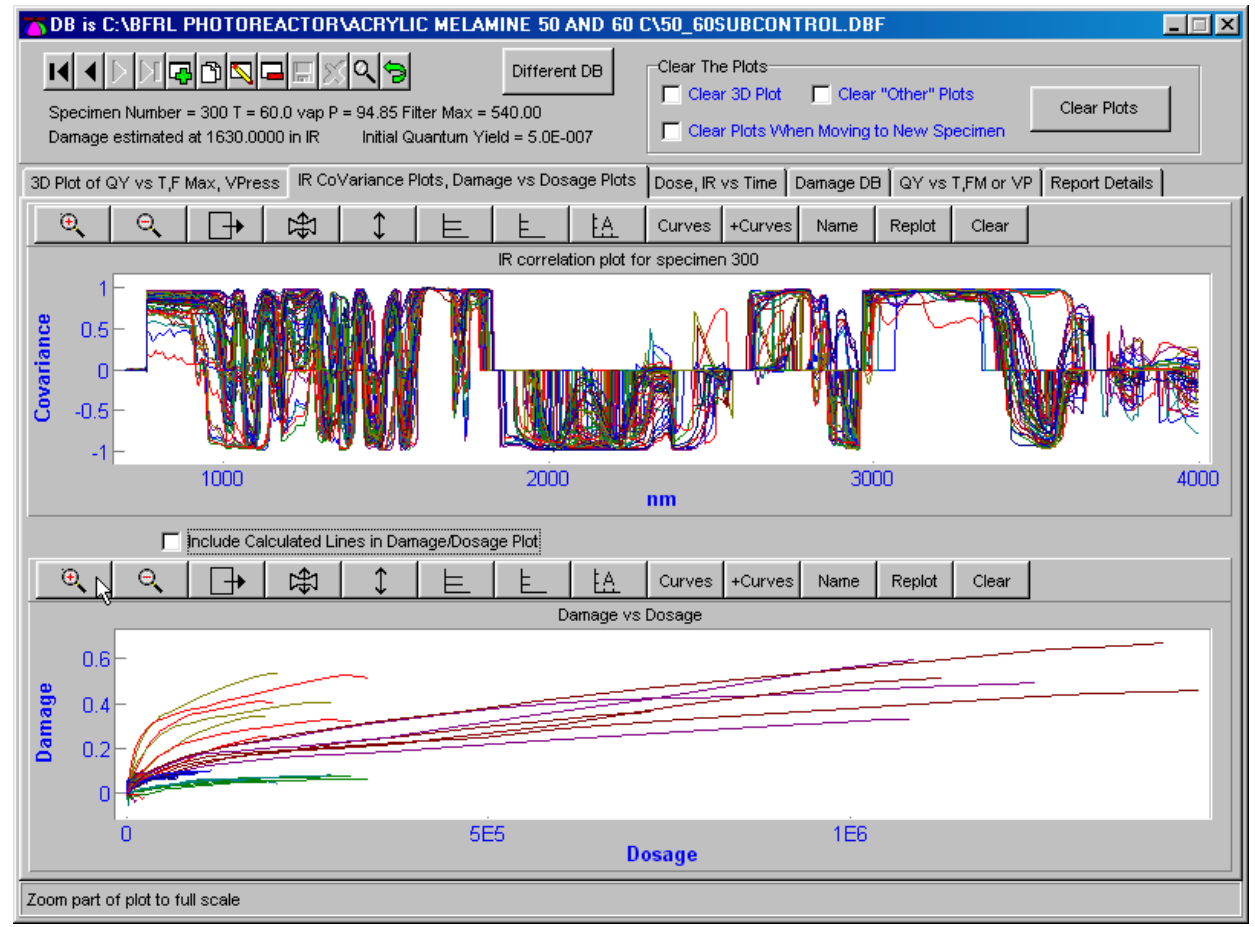

The plot was zoomed (using the first plot button) and the short lines identified using the plot "Names" button. As shown, one of the short lines is for the wavelength of $290 \mathrm{~nm}$. 


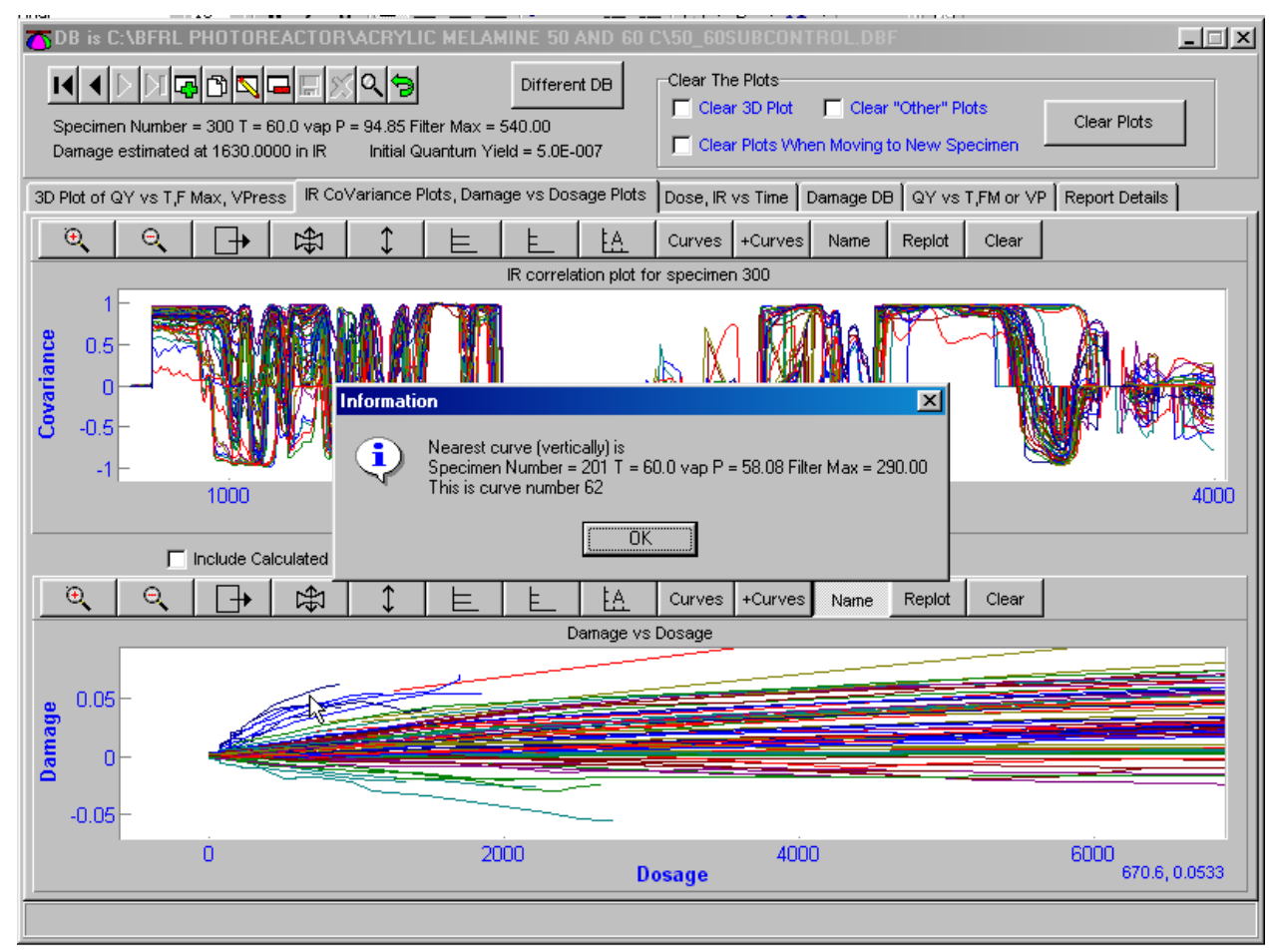

The following section of page 6 of the LookAtDamage program contains instructions on how to choose the conditions for which to plot the quantum yields, and also allows the user to join the plotted points with a line of the same color as the points (this is very useful if the data seem to be filling the plot haphazardly).

Choose Damage Site from box on right, then Fitter Wavelength then Temperature, then Vapor Press. Then click on plot button. Combo boxes must be chosen starting at top.

Г Connect Pts With Line

ॠ Add Curves To Other Plots

Print Report

$\sqrt{\checkmark}$ Clear Other Plots When This Plot Cleared

$\Gamma$ Change to Micromols of Photons

The check box "Add Curves To Other Plots" means that dosage versus time, damage versus time, correlations, and damage versus dosage curves will be added to the plots on pages 2 and 3 . Those plots will be cleared when this plot is cleared if the appropriate check box is checked (see above).

The "Change to Micromols of Photons" checkbox uses the wavelength of the filter maximum transmission to convert dosage stored as Joules into photons. Note that quantum yields estimated from damage at a given number of absorbed Joules are not the same as those estimated 
for a given number of photons. It is probably better to estimate quantum yields at a given number of absorbed photons, bearing in mind that these quantum yields are only applicable to the whole degradation process when the trend of damage is linear with dosage. This is usually not the case. The importance of the quantum yields is that they allow comparison of the effects of degradation conditions to be made for the early part of the degradation and provide a mechanism for assessing whether the degradation results make sense.

The print report button allows the user to get a hard copy version of the plot, perhaps for group discussions. The program window can be captured to the clipboard by simultaneously pressing Alt-PrtSc.

The report screen is show below and allows the user to document the report to some extent.

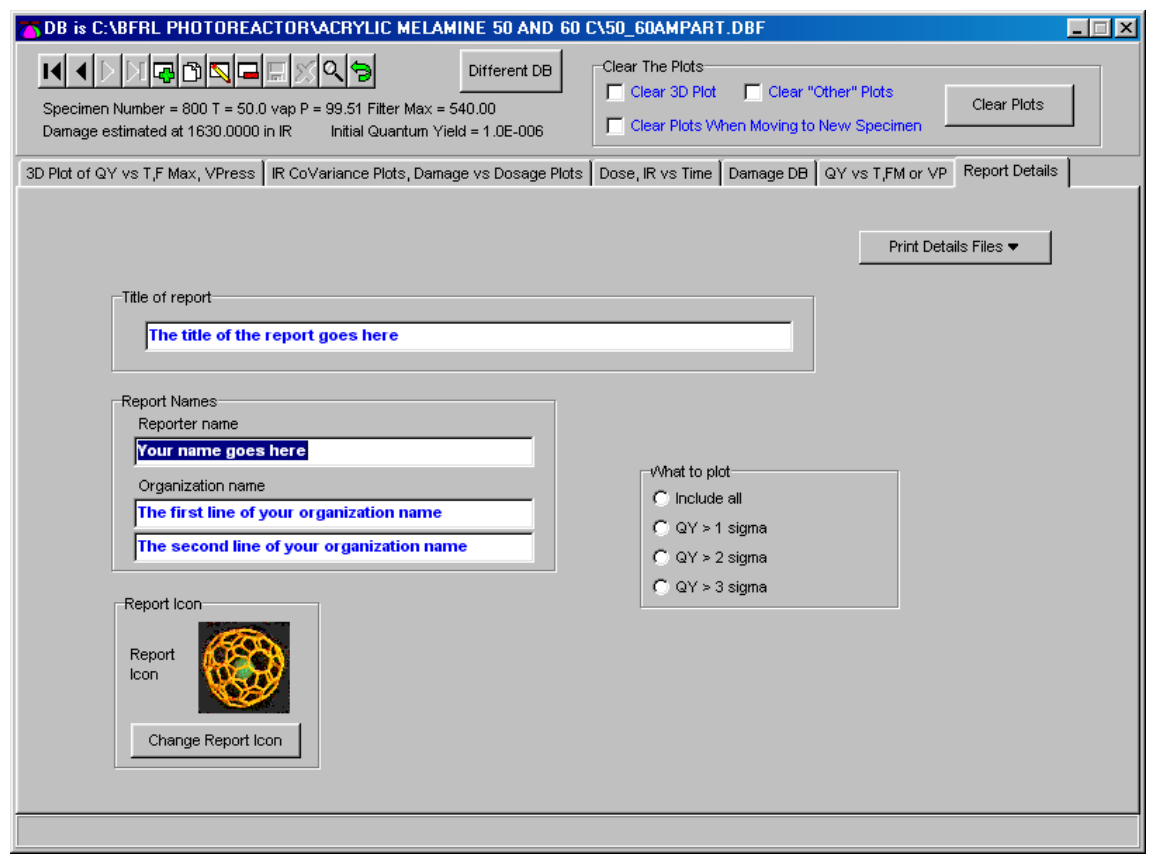

\section{SUMMARY}

This project has now advanced to the point where it has provided a highly automated apparatus that generates data for a wide range of conditions. The system of programs induces discipline in the experimental protocol, automates our expertise, and allows archival storage of data in readily accessible form.

The combination allows greater productivity and provides enormous capability which now exists in a form which can readily be used by others. Using this capability requires due care and attention to detail. In an activity as large and complex as the system described here, the interactions of a wide range of conditions and complicated phenomena must be understood and treated correctly. There is a tremendous 
amount of data to examine, track, recall and use. These data will refine our perceptions of what has to be controlled better, what can be calculated, how results can be compared, and how the chemistry proceeds, in that order.

\section{Recommendations:}

\section{Light Source}

- use a <290nm cut-off filter

- brighten the light sources and lengthen the exposures until one can interpolate on dosage, not extrapolate, when relating dosage and damage and extrapolating to field conditions

- pay particular attention that the reactions are not accelerated so much that they are starved of reactants (e.g., oxygen)

\section{UV Spectra}

- frequent calibration of the spectrometers to get acceptable stability over long times

- measure low absorbance/irradiance regions with great precision

- use standard samples to document the performance of the spectrometers at the time the spectra are measured.

\section{IR Spectra}

- use IR SRM or "standard" (PS) film to calibrate the spectrometer when spectra are measured

- use samples of various thickness to find range of good absorbance in spectra

- all absorbance spectra are ratios. Both parts of the ratio must be correct. Incorporate the measurements in the programs immediately so that comparisons with the others in the series can be used to help to detect misfits/errors.

- save components of the ratios used to calculate the spectra (?)

\section{Experimental Considerations}

\section{Noise}


Noise comes from measuring

- the spectra

- $\quad$ specimen thickness $(\mathrm{S} / \mathrm{N})$

and more error comes from:

- time-based drift of the measurement systems.

\section{Dosage}

The dosage depends on the radiation flux and the specimen UV absorbance spectrum. The dosage is expected to be significant when there is:

- high radiation flux, high spectral absorbance

- high radiation flux, low spectral absorbance

- low radiation flux, high spectral absorbance

The specimen absorbance must be measured better where the radiation flux is high than where the radiation flux is low.

The radiation flux must be measured better where the specimen absorbance is high.

\section{Processed spectra}

Specimen UV spectra should change systematically (which usually will mean monotonically) during exposure

After subtraction of dark reactions (hydrolysis, post cure), the results (in the IR spectra) should probably be monotonic with dosage - if not, either the subtraction process was not satisfactory or the kinetics include both production and consumption of the chemical group.

Depending on the aspect of interest, corrections for ablation/erosion should or should not be carried out. Ablation is very important because the coating was originally applied for good reason. Correcting for ablation will make the chemical changes more prominent and will be helpful in elucidating the degradation mechanisms. Not correcting for ablation will show how quickly the film is disappearing. 


\section{Miscellaneous Considerations}

Do degradation products shield the specimen from degradation by absorbing some of the incident radiation? This effect can be treated in the dosage calculating program. The dosage calculations can be carried out with and without "partitioning" the dosage into that absorbed by the original matrix and that absorbed by degradation products. If partitioning is more realistic than not partitioning, this will appear to give more reasonable plots such as the trend of quantum yield with wavelength when results from different conditions and from specimens with different ratios of matrix and degradation products are compared.

The spectral measurements will be will invalid if

- A significant amount of radiation was scattered or otherwise prevented from reaching the detector without being absorbed by the specimen.

- The specimen delaminated from its supporting plate, creating an air interface which introduced back reflections

- The supporting plates crazed during the experiments

\section{Appendix 1. Structure of the Database Tables}

The first column in the following description of the database tables is the name given to the field in the database table and can contain up to 11 characters. The second column is the type of field: C means characters, $\mathrm{N}$ means a number, and $\mathrm{L}$ means logical, i.e., true or false (in later versions, this was changed to a character field where $\mathrm{T}=$ true and $F=$ false). The third column is the length of the field (in terms of the maximum number of characters it can contain). The fourth column is the number of "sub-digits". This is the number of digits after the decimal point if the field contains a non-integer number.

\section{Suppliers}

$\begin{array}{llll}\text { SUPPL_NUM } & \text { C } & 15 & 0 \\ \text { FIRM_NAME } & \text { C } & 40 & 0 \\ \text { ADDRESS1 } & \text { C } & 40 & 0 \\ \text { ADDRESS2 } & \text { C } & 40 & 0 \\ \text { CITY } & \text { C } & 20 & 0 \\ \text { STATE_ETC } & \text { C } & 20 & 0 \\ \text { COUNTRY } & \text { C } & 20 & 0 \\ \text { CONT_TITLE } & \text { C } & 10 & 0 \\ & & & \end{array}$




$\begin{array}{llll}\text { CONT_1ST } & \text { C } & 20 & 0 \\ \text { CONT_MI } & \text { C } & 5 & 0 \\ \text { CONT_LAST } & \text { C } & 20 & 0 \\ \text { PHONE } & \text { C } & 20 & 0 \\ \text { PHONE_EXT1 } & \text { C } & 6 & 0 \\ \text { PHONE_EXT2 } & \text { C } & 6 & 0 \\ \text { FAX } & \text { C } & 20 & 0 \\ \text { WWW } & \text { C } & 30 & 0 \\ \text { EMAIL } & \text { C } & 30 & 0 \\ \text { REMARKS } & \text { C } & 254 & 0\end{array}$

The meaning of most of these fields may be guessed. The CONT fields refer to the contact at the company - his title, first name, middle initial and last name. Some of the field names appear to be cryptic but this is a consequence of the field name having to be less than 12 characters.

\section{Lamp ID}

$\begin{array}{llll}\text { LAMP_NUM } & \text { C } & 15 & 0 \\ \text { ID } & \text { C } & 80 & 0 \\ \text { SPECS } & \text { C } & 254 & 0 \\ \text { SUPPLR_NUM } & \text { C } & 15 & 0 \\ \text { PURCH_DATE } & \text { C } & 10 & 0\end{array}$

The Lamp_Num field is used to relate the position in the illumination field of the lamps to a specific lamp. A description may be given in the ID field and detailed specifications may be stored in the SPECS field. The SUPPLR_NUM field uses the user-assigned supplier number to point to a record in the Supplier table.

\section{Cell ID}

$\begin{array}{llll}\text { CELL_NUM } & \text { C } & 15 & 0 \\ \text { ID } & \text { C } & 80 & 0 \\ \text { SPECS } & \text { C } & 254 & 0 \\ \text { SUPPLR_NUM } & \text { C } & 15 & 0 \\ \text { PURCH_DATE } & \text { C } & 10 & 0\end{array}$

The Cell ID table allows the user to enter some information about a particular cell, given its cell number.

\section{Filter ID}




$\begin{array}{llll}\text { FILTR_NUM } & \text { C } & 15 & 0 \\ \text { ID } & \text { C } & 80 & 0 \\ \text { SPECS } & \text { C } & 254 & 0 \\ \text { SUPPLR_NUM } & \text { C } & 15 & 0 \\ \text { PURCH_DATE } & \text { C } & 10 & 0 \\ \text { FILTER_MAX } & \text { N } & 10 & 2 \\ \text { HALF_WIDTH } & \text { N } & 10 & 2 \\ \text { UNITS } & \text { C } & 15 & 0\end{array}$

The main use of the Filter ID table is to allow the program to find the transmission characteristics of the filter from knowing the filter number. The Filter_Max field is the center wavelength of the transmission window of the filter and the Half_Width field is the width of the filter band-pass at half maximum. The units of the filter wavelength are given in the Units field and should be the same as the $x$ axis units of the UV-visible spectra.

\section{Specimen ID}

$\begin{array}{llll}\text { SPEC_NUM } & \text { C } & 15 & 0 \\ \text { ID } & \text { C } & 80 & 0 \\ \text { SUPPLR_NUM } & \text { C } & 15 & 0 \\ \text { THICKNESS } & \text { N } & 6 & 1 \\ \text { SOURCE } & \text { C } & 254 & 0 \\ \text { NOTES } & \text { C } & 254 & 0\end{array}$

The Specimen ID table allows the user to give a specimen a number and to key this specimen to a supplier. The program will use the table to get the specimen thickness for a particular specimen number.

Specimen $\mathbf{0}$ is used to hold the thickness of a thick specimen which is included solely to provide a good measurement of the material's UV-visible spectrum.

\section{Cell Position}

$\begin{array}{llll}\text { CELL_NUM } & \text { C } & 15 & 0 \\ \text { POSITION } & \text { C } & 15 & 0 \\ \text { FILTER1 } & \text { C } & 15 & 0 \\ \text { FILTER2 } & \text { C } & 15 & 0 \\ \text { FILTER3 } & \text { C } & 15 & 0 \\ \text { FILTER4 } & \text { C } & 15 & 0 \\ \text { FILTER5 } & \text { C } & 15 & 0 \\ \text { TEMPERAT } & \text { N } & 6 & 1\end{array}$




$\begin{array}{llll}\text { HUMIDITY } & \mathrm{N} & 6 & 1 \\ \text { WHEN } & \mathrm{N} & 10 & 4\end{array}$

Each cell may in principle have more than one filter and is kept at some specified temperature and humidity. When the specimen is placed in a cell, the cell number keys the above quantities to the specimen. In practice, only one filter has been used and the programs are now not set up to handle more than one filter per cell.

The POSITION field keys the cell to a position in the illumination field of the lamps.

The WHEN field is the date and time the cell was placed in its position. The date and time are entered into the programs as normal dates and times but are transformed by the program into the number of days (and fraction of days) since the start of the $20^{\text {th }}$ century by the program before being written to the database tables.

\section{Specimen In Cell}

$\begin{array}{llll}\text { SPEC_NUM } & \text { C } & 15 & 0 \\ \text { CELL_NUM } & \text { C } & 15 & 0 \\ \text { WHEN } & \text { N } & 10 & 4\end{array}$

This table allows the program to relate the specimen number to a cell number so that it knows from the cell position field which lamp spectra should be used for the irradiance and which filters were placed between the specimen and the lamp. The cell position table also provides the temperature and humidity at which the exposure was conducted.

\section{Lamps On/Off}

$\begin{array}{llll}\text { LAMP_NUM } & \text { C } & 15 & 0 \\ \text { ON } & \text { C } & 1 & 0 \\ \text { WHEN } & \text { N } & 10 & 4\end{array}$

The lamp, some of the filters, and many of the specimens change in UV absorption characteristics with time, but spectra are taken sufficiently frequently for these changes to be followed by linear interpolations between spectra. To take the spectra, the lamp must be switched off. This table documents the lamp on/off times so that the total time the lamp was on can be calculated. Note that when the lamp is turned on, the entry in this table has a T in the ON field. When the lamp is turned off, ON is false, so the ON field contains $F$.

\section{Spectrum}




$\begin{array}{llll}\text { WHICH } & \mathrm{C} & 15 & 0 \\ \text { WHEN } & \mathrm{N} & 10 & 4 \\ \text { X0 } & \mathrm{N} & 6 & 0 \\ \text { DELTA_X } & \mathrm{N} & 6 & 0 \\ \text { NUM_PTS } & \mathrm{N} & 6 & 0 \\ \text { SPECTRUM_Y } & \mathrm{M} & 10 & 0\end{array}$

Four types of spectra have been used up to the present time. They are the lamp UV spectra, the filter transmission UV spectra, the specimen UV absorption spectra, and the specimen IR spectra. The TYPE_NUM is 1 for lamps, 2 for filters, 3 for specimen UV, and 4 for specimen IR spectra. The WHICH field specifies the lamp, filter or specimen to which this spectrum belongs by containing the appropriate LAMP_NUM, FILTR_NUM or SPEC_NUM. The WHEN field denotes when this spectrum was taken and is very important in showing to which lamp-off time-slice the spectrum belongs. The UV-visible spectrum of specimen 0 is the spectrum of the thick standard specimen.

Because the spectra are equally spaced on the wavelength axis (x), only the starting $x$, the spacing along $x$ and the number of points need be supplied. For example, $X 0=290$ and DELTA_X $=2$ means that the starting wavelength is 290 and adjacent spectral values are spaced by 2 wavelength units. This saves a considerable amount of space in the database table. The $y$ values of the spectra are however all different so each y value must be given. The y values are stored as a memo/BLOB (binary large object) which is a memory dump of the array of $y$ values from the computer memory to the database table on the disk. When this BLOB is read back into the computer from the database table, it occupies the same memory configuration as before and may be referenced immediately by the program as though it had never been disturbed. The spectral files are written from CSV or JCAMP files using the ReadSpectra program.

\section{Cell Position To Lamp}

$\begin{array}{llll}\text { POSITION } & \text { C } & 15 & 0 \\ \text { LAMP_NUM } & \text { C } & 15 & 0 \\ \text { WHEN } & \text { N } & 10 & 4\end{array}$

Each cell is placed in an illumination field and thus has an associated lamp, found via the Lamp_Num, and a position in the field irradiated by the lamp. The intensity of the received radiation depends on the position the cell occupies in the illumination field. A lamp spectrum must be available for each position in the lamp illumination field. The WHEN field documents when the cell was placed in the lamp field.

The POSITION field provides an identification for each position illuminated by the lamp specified in LAMP_NUM. Lamp spectra are measured at each exposure position. 


\section{Lamp Power}

$\begin{array}{llll}\text { WAVELNGTH } & \text { N } & 10 & 4 \\ \text { PWRFACTOR } & \text { N } & 10 & 4 \\ \text { WHEN } & \text { N } & 10 & 4\end{array}$

The lamp power as read by the spectrometer is in somewhat arbitrary units which are placed on a scale of watts using the wavelength-specific conversion factors found in the Lamp Power table.

\section{Derived quantities}

\section{Damage}

\begin{tabular}{|c|c|c|}
\hline SPEC_NUM & $\mathrm{C}$ & 15 \\
\hline DAMAGE_NUM & $\mathrm{N}$ & 10 \\
\hline FILTER_MAX & $\mathrm{N}$ & 10 \\
\hline CNTRL_FIL & $\mathrm{N}$ & 10 \\
\hline HALF_WIDTH & $\mathrm{N}$ & 10 \\
\hline TEMPERAT & $\mathrm{N}$ & 6 \\
\hline HUMIDITY & $\mathrm{N}$ & 6 \\
\hline VAP_PRESS & $\mathrm{N}$ & 10 \\
\hline INIT_QY & $\mathrm{N}$ & 16 \\
\hline ABLAT_SITE & $\mathrm{N}$ & 10 \\
\hline MINUS_HYD & $\mathrm{C}$ & 1 \\
\hline NO_ABLAT & $\mathrm{C}$ & 1 \\
\hline PARTN_UV & C & 1 \\
\hline SUB_CNTRL & C & 1 \\
\hline SCALE2THCK & C & 1 \\
\hline SMOOTH & C & 1 \\
\hline DELTA_IR & $\mathrm{N}$ & 10 \\
\hline DELTA_UV & $\mathrm{N}$ & 10 \\
\hline MAX_TIME & $\mathrm{N}$ & 10 \\
\hline MAX_DOSE & $\mathrm{N}$ & 10 \\
\hline MAX_DAMAGE & $\mathrm{N}$ & 10 \\
\hline NUM_EQCOEF & $\mathrm{N}$ & 4 \\
\hline Q_EQUATION & $\mathrm{M}$ & 10 \\
\hline ABLATION & $\mathrm{M}$ & 10 \\
\hline IR_X0 & $\mathrm{N}$ & 6 \\
\hline
\end{tabular}




$\begin{array}{llll}\text { IR_DELTA_X } & \mathrm{N} & 6 & 0 \\ \text { R_NUM_PTS } & \mathrm{N} & 6 & 0 \\ \text { CORRELAT_Y } & \mathrm{M} & 10 & 0 \\ \text { DOSAGE_X } & \mathrm{M} & 10 & 0 \\ \text { DAMAGE_Y } & \mathrm{M} & 10 & 0 \\ \text { DELUV_ABSX } & \mathrm{M} & 10 & 0 \\ \text { DELUV_ABSY } & \mathrm{M} & 10 & 0 \\ \text { DELIR_TIME } & \mathrm{M} & 10 & 0 \\ \text { DELIR_ABSY } & \mathrm{M} & 10 & 0 \\ \text { DOSE_TIME } & \mathrm{M} & 10 & 0 \\ \text { DOSE_Y } & \mathrm{M} & 10 & 0 \\ \text { BAND_WIDTH } & \mathrm{N} & 10 & 2 \\ \text { FORM_WT } & \mathrm{N} & 10 & 1 \\ \text { ORIG_WHEN } & \mathrm{N} & 10 & 4 \\ \text { WHEN_CALC } & \mathrm{N} & 10 & 4 \\ \text { DENSITY } & \mathrm{N} & 10 & 4 \\ \text { THICKNESS } & \mathrm{N} & 6 & 1 \\ \text { LAMP_ZERO } & \mathrm{N} & 10 & 4 \\ \text { UV_ZERO } & \mathrm{N} & 10 & 4 \\ & & & \end{array}$

The damage database table contains many derived quantities and some quantities which have been copied from the other tables. It is generated by the data-processing program to be described later. Its contents are not as fully established as the other tables and may change as the requirements of the technique evolve.

The "main" entries are

SPEC_NUM the specimen number

DAMAGE_NUMthe damage site in the damage spectrum

FILTER_MAX the UV-visible filter used

CNTRL_FIL the filter (if any) of the control

HALF_WIDTH the half width of the UV-visible filter

TEMPERAT the temperature of the exposure

HUMIDITY the relative humidity of the exposure

VAP_PRESS the water vapor pressure of the exposure

INIT_QY the initial quantum yield

ABLAT_SITE the site in the damage spectrum used to correct for specimen thickness and ablation

LAMP_ZERO the wavelength at which the lamp spectra were set to zero 
UV_ZERO

MINUS_HYD

NO_ABLAT

PARTN_UV

SUB_CNTRL

SCALE2THCK

SMOOTH the wavelength at which the specimen spectra were set to zero

$\mathrm{T}=$ hydrolysis was subtracted, $\mathrm{F}=$ hydrolysis was not subtracted

$T=$ the results do not contain the effects of ablation, $\mathrm{F}=$ the results do contain the effects of ablation

$T=$ the dosage was partitioned according to the initial UV absorbance of the specimen and the actual UV absorbance as the degradation progressed

$\mathrm{T}=$ control spectra were subtracted from the specimen spectra, $\mathrm{F}=$ control spectra were not subtracted from the specimen spectra

$\mathrm{T}=$ all spectra were scaled to a common thickness before subtractions, $\mathrm{F}=$ spectra were used without scaling for thickness differences

$\mathrm{T}=$ all spectra were smoothed before subtractions, $\mathrm{F}=$ spectra were used without smoothing

\section{Indexing of database tables}

The information in a database table is written to a disk file in the form of records, each record being a line of information. The order in which the information is written to the disk in general does not matter because the information is retrieved from the tables by "indexing" them. This means that a sort condition is imposed on the table. For example, perhaps the order should be that the records be found according to increasing values in the WHEN (date and time) field or column. The records are never physically re-ordered. Instead, an index is computed that tells the program the order in which to read the records to achieve the desired result. In the programs described in this guide, the index is computed anew when the database table is first read into the program. Index computation is remarkably fast, thanks to fast computers, large computer memories and buffered hard disks, where swathes of information are read from the disk and kept in a buffer until a new region of the disk is to be read.

The order of records when the WHEN column (field) is ordered is kept in the index file and is imposed on the table by setting the index tag, which is the name of the index condition. As an example, if we have a list of numbers,

$500,700,300,200,100$

and sort them into increasing order, we would take the 5th number, then the 4 th, then the $3 \mathrm{rd}$, then the $1 \mathrm{st}$, then the 2 nd number. Our index would then be $5,4,3,1,2$. If the program wanted the first member of this series, it would get the 5th number. Because of the definitions of the field lengths, the program knows how long each number is in terms of characters, so it does not have to read the first 4 numbers to get to the 5 th. It merely determines where on the disk the 5th number is, goes 
there, and reads the number of characters which it knows represent the required number. If we want to show all the numbers sorted on increasing magnitude and with values between 200 and 400, that is done in a similar fashion.

Database tables are used in exactly this way. A condition is applied, perhaps all spectra between two dates for position 103 in the lamp illumination field. Only those spectra which fit the condition are visible to the program, which can then read the spectrum table from top to bottom to get the spectra in terms of increasing time.

The indexes computed in the programs are given below. The name given to the index condition is the index tag name, and the actual condition is the index field name, so-called because it is based on the values in the fields. STR means a number has been converted into an alphanumeric form.

\title{
Lamp ID
}

Index Tag Name 1 = LAMPNUM

Index Field Name 1 = LAMP_NUM

\section{Cell ID}

Index Tag Name 1 = CELLNUM

Index Field Name 1 = CELL_NUM

\section{Filter ID}

\author{
Index Tag Name 1 = FILTNUM \\ Index Field Name 1 = FILTR_NUM; \\ Index Tag Name 2 = FILTMAX \\ Index Field Name 2 = FILTER_MAX;
}

\section{Suppliers}

\author{
Index Tag Name 1 = SUPPLNUM; \\ Index Field Name 1 = SUPPL_NUM;
}




\section{Specimen ID}

Index Tag Name 1 = SPECNUM;

Index Field Name 1 = SPEC_NUM;

\section{Specimen In Cell}

Index Tag Name 1 = SPECIMEN;

Index Field Name 1 = SPEC_NUM;

Index Tag Name 2 = SPECWHEN;

Index Field Name 2 = SPEC_NUM+STR(WHEN,10,4);

Index Tag Name 3 = CELL;

Index Field Name 3 = CELL_NUM;

\section{Cell Position}

Index Tag Name 1 = CELL;

Index Field Name 1 = CELL_NUM;

Index Tag Name 2 = CELLTempHum;

Index Field Name $2=$

CELL_NUM+STR(TEMPERAT,6,1)+STR(HUMIDITY,6,1);

Index Tag Name 3 = CELLWHEN;

Index Field Name 3 = CELL_NUM+STR(WHEN,10,4);

Index Tag Name 4 = TempHum;

Index Field Name 4 = STR(TEMPERAT,6,1)+STR(HUMIDITY,6,1);

\section{Lamps On/Off}

Index Tag Name 1 = ONOFF;

Index Tag Name 2 = TIMES;

Index Field Name 1 = LAMP_NUM+ON+STR $($ WHEN, 10,4)

Index Field Name 2 = LAMP_NUM+STR(WHEN, 10,4); 


\section{Spectrum}

Index Tag Names 1 = SPECT_BOTH

Index Field Name $1=$ WHICH + STR(WHEN, 10,4)

\section{Cell Position To Lamp}

Index Tag Names 1 = POSITNWHEN

Index Field Name 1 = POSITION + STR(WHEN,10,4)

\section{Lamp Power}

Index Tag Name 1 := LMPWAVWHEN

Index Field Names 1 = LAMP_NUM + STR(WAVELNGTH,10,4) + STR(WHEN,10,4)

\section{Damage}

Index Tag Name 1 = DAMFTV;

Index Field Name $1=$ STR(DAMAGE_NUM, 10,4) + STR(FILTER_MAX,10,2) + STR(TEMPERAT,6,1) + STR(VAP_PRESS, 10,4)

Index Tag Name 2 = DAMTVF

Index Field Names 2 = STR(DAMAGE_NUM,10,4) + STR(TEMPERAT,6,1) + STR(VAP_PRESS, 10,4) + STR(FILTER_MAX,10,2)

Index Tag Name 3 = DAMVTF

Index Field Name $3=$ STR(DAMAGE NUM, 10,4) + STR(VAP_PRESS, 10,4) + STR(TEMPERAT, 6,1) + STR(FILTER_MAX,10,2)

The damage table can be sorted in three ways. In each case, the damage site is the most slowly varying column. The choices are then which of the remaining columns varies the next most slowly, and which is the fastest varying. Not all possibilities are covered. 


\section{Appendix 2: Relative Humidity and Vapor Pressure of water}

The saturation water vapor pressure at a given temperature shows how much water can be absorbed/dissolved by air at that temperature. The ranges of water vapor pressure for temperatures experienced in outdoor exposures overlap less than might be expected. For example, water vapor with a dew point of $20 \mathrm{C}$ (i.e., saturated at that temperature) is only $11 \% \mathrm{RH}$ (relative humidity) at $60 \mathrm{C}$.

The relationship between the amount of water vapor in the atmosphere, the temperature, and the amount of water absorbed in a solid film specimen is complex. As the temperature increases, the relative humidity of the atmosphere will decrease and the ability of the atmosphere to take up more water will increase. If no more water is introduced into the system, the film will be less likely to hold water because its absorbed water is in the condensed state in an amount in equilibrium with the former lower temperature. Under these conditions, there will be a loss of water from the film to the atmosphere. The change in water-content in the film will depend on the relative waterdissolving (or absorbing) powers of the film and atmosphere at the new temperature but will qualitatively be in the same direction as the changes in relative humidity, i.e, as the relative humidity increases, the water content of the film will increase (see below). The kinetics of hydrolysis depend on the water concentration (expressed in moles/L in kinetics) in the film specimen.

More water in the atmosphere (higher relative humidity) means:

a) higher equilibrium concentration of water in the specimen, and

b) faster attainment of a considerable concentration of water in the specimen.

Liquid water has more than a thousand times higher concentration of water than does water vapor. When the equilibrium state of water is liquid water, fissures, pores and cracks in the film will fill with liquid water. Any water which is consumed in hydrolysis reactions will rapidly be replaced. If hydrolysis is found to be a significant degradation mechanism using water vapor, this suggests that, for outside exposures:

a) most degradation takes place during rainy periods and below the dew point at night and in the early morning, when liquid water is widely available, and

b) the sun coming out is the most beneficial thing that could happen.

\section{Temperature/vapor pressure table}

We can compare the relative concentrations of water in vapor and liquid forms.

Water has a molecular weight (MW) of 18. A liter of liquid water contains $1000 \mathrm{~g} / 18 \mathrm{~g} / \mathrm{MW}=55.55$ moles. 
Water vapor pressure at the saturation level, i.e., in equilibrium with liquid water, varies with temperature as in the table below. From the vapor pressure of the water vapor, we can calculate the fraction of a liter of saturated air that is water vapor, assuming that the atmospheric pressure is $760 \mathrm{~mm} \mathrm{Hg}$. From the concept that a formula weight of an ideal gas occupies $22.4 \mathrm{~L}$ at STP, we can estimate the moles/L of water in saturated air at the various temperatures. Then we can compare the relative concentrations of liquid water and water-saturated air. There is several thousand times more water per liter in liquid water than in moist air.

$\begin{array}{cccccc}\mathrm{T},{ }^{\circ} \mathrm{C} & \mathrm{mm} \mathrm{Hg} & \text { Liters vapor } & \begin{array}{c}\text { Molar } \\ \text { Volume }\end{array} & \text { Mole/L water } & \begin{array}{c}\text { Mole/L ratio, } \\ \text { liquid to vapor }\end{array} \\ -10 & 2.15 & 0.0028 & 19.77 & 0.00014 & 388,231 \\ 0 & 4.58 & 0.0060 & 20.52 & 0.00029 & 189,178 \\ 10 & 9.21 & 0.0121 & 21.27 & 0.00057 & 97,521 \\ 20 & 17.5 & 0.0231 & 22.02 & 0.00105 & 53,016 \\ 30 & 31.8 & 0.0419 & 22.78 & 0.00184 & 30,221 \\ 40 & 55.3 & 0.0728 & 23.53 & 0.00309 & 17,957 \\ 50 & 92.5 & 0.1217 & 24.28 & 0.00501 & 11,081 \\ 60 & 149 & 0.1966 & 25.03 & 0.00785 & 7,075 \\ 70 & 233 & 0.3075 & 25.78 & 0.01193 & 4,658 \\ 80 & 355 & 0.4672 & 26.53 & 0.01761 & 3,155 \\ 90 & 525 & 0.6918 & 27.29 & 0.02536 & 2,191 \\ 100 & 760 & 1.0000 & 28.04 & 0.03567 & 1,558\end{array}$

With higher temperatures, there is

a) a bigger range of available water vapor pressure

b) faster hydrolysis for a given water vapor pressure

c) more difficulty in assessing degradation other than hydrolysis because hydrolysis constitutes a greater part of the total damage, the remaining degradation is necessarily a smaller part of the total damage, and the noise depends on the total damage so, when the hydrolysis component has been subtracted, the remaining degradation must be discerned against a larger background of noise

$\mathrm{RH}$ is the percentage ratio of the actual vapor pressure of water to the saturated vapor pressure of water at that temperature. The vapor pressure of water vapor in equilibrium with liquid water varies widely with temperature and is exponentially sensitive to temperature. Because of the small overlap of the ranges of water vapor pressure at different temperatures, similar values of relative humidity at significantly different temperatures are actually based on very different levels of water vapor 
pressure. The programs transform relative humidity into water vapor pressure as well as recording the relative humdity.

We note also that water is an excellent coolant because it has a high latent heat of vaporization (about $580 \mathrm{cal} / \mathrm{g}$ at $25 \mathrm{C}$, about 10 times that of most liquids). Evaporating water therefore requires a lot of heat, which means, when generating a specific humidity level, that a plentiful supply of heat must be available and the temperature must be well-controlled. This consideration has been included in the design of the photoreactor.

\section{Partitioning of water between ambient air and specimens}

As described above, water in an atmosphere will partition between that atmosphere and any available substrate. For example, poly(methyl methacrylate), PMMA, takes up about $0.5 \%$ water by weight in air with $50 \% \mathrm{RH}$ at $25 \mathrm{C}$ and about $1.8 \%$ water by weight in $100 \% \mathrm{RH}$ air. The density of PMMA is about $1.2 \mathrm{~g} / \mathrm{mL}$, so $0.5 \%$ water in PMMA is about $0.33 \mathrm{~mole} / \mathrm{L}$, and $1.8 \%$ water in PMMA is about $1.2 \mathrm{~mole} / \mathrm{L}$. The concentration of water vapor in $100 \% \mathrm{RH}$ at $25 \mathrm{C}$ ambient air is, from the table above, about $0.0015 \mathrm{~mole} / \mathrm{L}$. The presence of polar groups such as ester groups in the PMMA has concentrated the water by factors of about 220 in $50 \% \mathrm{RH}$ air and about 800 in $100 \% \mathrm{RH}$ air. $1.8 \%$ water corresponds to roughly 1 water molecule for every 10 ester groups. This trend in absorption of water is not linear with relative humidity. Given that the water absorbed at low relative humidity is hydrogen-bonded to ester groups but water only absorbed at high relative humidity is probably hydrogen-bonded to water already in the specimen, it is not clear that it should be. On the other hand, our unpublished measurements of the water content at $25 \mathrm{C}$ of polyimide films and of polyimide disks containing silica filler do show a linear relationship of water-uptake with $\mathrm{RH}$.

To know how much water is actually present in specimens being degraded in humid environments, one must measure the partitioning of water between the ambient air and the specimen as a function of water vapor pressure, temperature, and, since polar groups are formed in the oxidation reactions of degradation, of extent of degradation. We have not yet performed these measurements.

Without knowledge of the absorption isotherms of water in the specimens, it is impossible to transform from $\mathrm{RH}$ in the atmosphere to concentration of water in the specimens. Without knowledge of the concentration of water in the specimens, results from different $\mathrm{RH}$ levels can not really be compared meaningfully and kinetic rate constants can not be calculated for the hydrolysis reactions. None the less, pending the establishment of a more rigorous approach and the measurement of the parameters required to implement the approach, provision has been made in the LookAtIR program for interpolation or extrapolation of damage estimates to different levels of $\mathrm{RH}$ to allow some sort of comparison to be made now. 